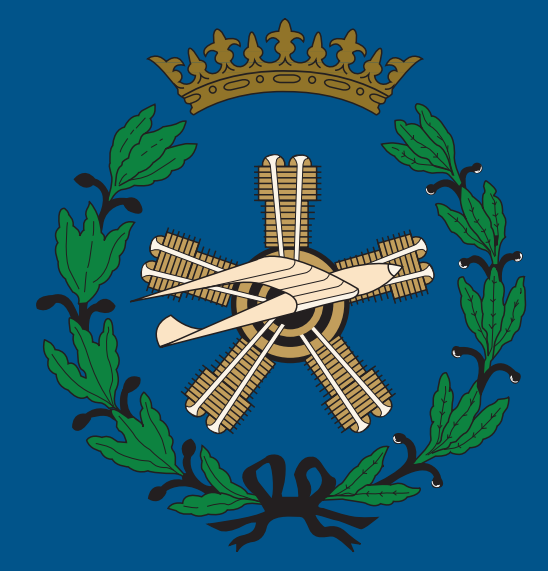

UNIVERSIDAD POLITÉCNICA DE MADRID

ESCUELA TÉCNICA SUPERIOR DE INGENIEROS AERONÁUTICOS

Doctoral Thesis:

\title{
FLUID-STRUCTURE INTERACTION OF CIRCULAR AND SQUARE CYLINDERS. DYNAMICS AND ENERGY TRANSFER
}

\author{
Ji Xu Xu \\ Ingeniero Aeronáutico
}

Madrid, 2017 



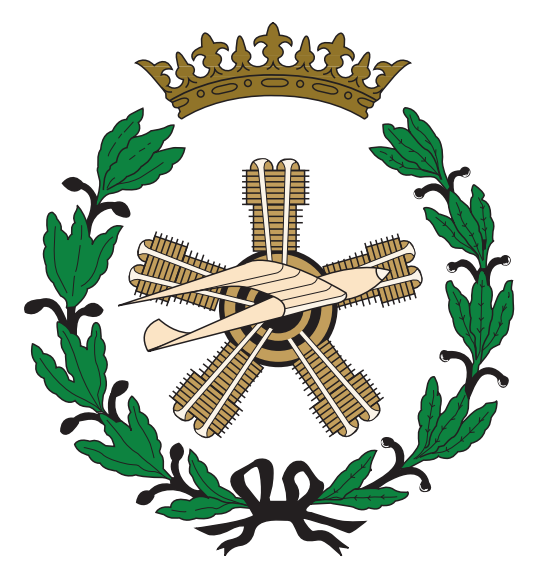

UNIVERSIDAD POLITÉCNICA DE MADRID

ESCUELA TÉCNICA SUPERIOR DE INGENIEROS AERONÁUTICOS

Doctoral Thesis:

\title{
FLUID-STRUCTURE INTERACTION OF CIRCULAR AND SQUARE CYLINDERS. DYNAMICS AND ENERGY TRANSFER
}

\author{
Ji Xu Xu \\ Ingeniero Aeronáutico
}

Madrid, 2017 

Departamento de Mecánica de Fluidos y Propulsión Aeroespacial

\title{
ESCUELA TÉCNICA SUPERIOR DE INGENIEROS AERONÁUTICOS
}

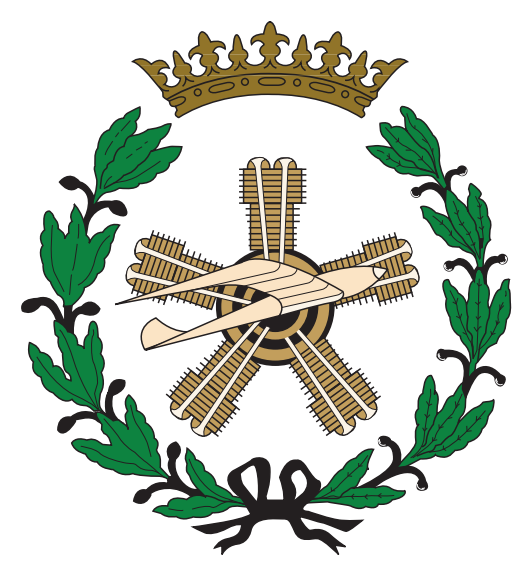

FLUID-STRUCTURE INTERACTION OF CIRCULAR AND SQUARE CYLINDERS. DYNAMICS AND ENERGY TRANSFER

Doctoral Thesis

\author{
Author: \\ Ji Xu Xu \\ Ingeniero Aeronáutico
}

Directed by:

Antonio Barrero Gil

Doctor Ingeniero Industrial

2017 



\section{POLITÉCNICA}

Tribunal nombrado por el Sr. Rector Magfco. de la Universidad Politécnica de Madrid, el día de de $20 \ldots$

Presidente:

Vocal:

Vocal:

Vocal:

Secretario:

Suplente:

Suplente:

Realizado el acto de defensa y lectura de la Tesis el día de. de $20 \ldots$ en la E.T.S.I. /Facultad.

Calificación

EL PRESIDENTE

LOS VOCALES 



\section{Acknowledgement}

Firstly, I would like to express my sincere gratitude to my Doctoral Thesis Director Prof. Dr. Antonio Barrero Gil for the continuous support of my PhD study and related research, for his patience, motivation, and immense knowledge. His guidance and encouragement helped me in all the time of research and writing of this Thesis, also for the hard question which incented me to widen my research from various perspectives. I could not have imagined having a better advisor and mentor for my PhD study.

My sincere thanks also goes to Prof. Dr. Ángel Velázquez and Prof. Dr. Juan Ramón Arias Pérez, who provided me an opportunity to join their research team as PhD candidate, and who gave access to the laboratory and research facilities. Without their precious support it would not be possible to conduct this research.

I will always be thankful to Miguel de las Heras, Ricardo de las Heras and José Sánchez for their continuous support in the laboratory, which made such outstanding experimental facilities possible. They would always surpass the desired quality of the required experimental hardware. I would also like to thank my fellow labmates, Miguel Reyes Mata and Francisco Sastre Matesanz with whom I have spent so many years and David Vicente Ludlam who was always willing to give me a hand whenever I needed.

This research has been funded by the international company REPSOL S.A., through the REPSOL-INSPIRE program and under contract P120130288. Through this program I was able to personally meet great professionals and people including Bernardo del Amo Fernández and Oscar Prieto Acedo.

Last but not the least, I would like to thank my family: my partner Lorenzo, my parents, Menghua and Fenfang and my brother, Roberto, for supporting me throughout this Thesis and my life in general. 



\section{Abstract}

Research on Flow-Induced Vibrations (FIV) of flexible bluff bodies under cross-flow is an important topic from both a scientific and a practical point of view. In recent years, FIV have raised interest both in civil engineering, where the purpose is the mitigation of FIV effects, and in the field of renewable energies, where the enhancement of energy transfer is sought. These apparently opposite applications underscore the necessity of an in-depth understanding of the FIV phenomena applicable to the design and the performance parametrization of structures and devices subjected to FIV.

However, theoretical models available in the literature seem to be incomplete and too restrictive for their application in FIV energy harvesting. This Thesis aims to expand and deepen knowledge in this field through fundamental studies and experimental research of the FIV phenomenology and its practical applications as a renewable power source.

For bluff bodies, there are two main classical types of FIV: Vortex-Induced Vibrations (VIV) where vortex shedding is synchronized with the natural frequency of the body; and Transverse Galloping (TG), a motion-induced instability often described by Quasi-steady (QS) models, which, in order to be applicable, require the assumption of slow oscillations when compared to the flow's characteristic convective time.

In the first approach to the comprehension of $\mathrm{TG}$, experiments were carried out in a recirculated free-surface water channel measuring transverse flow-induced oscillations of a low mass ratio square-section cylinder with low mechanical damping. The experimental results not only enlarged the existing published data, but also allowed to gain new insight into the phenomena: a new dimensionless parameter, a 'true' reduced velocity, provided an original re-normalization plot of the steady-state amplitude of oscillations for the first time in the literature. This discovery allows determining the velocity at which oscillations are expected to start and how they correlate to a sinusoidal oscillation as well as establishing the validity of QS conditions.

The hypothesis that the flow around the body at time $t$ has not enough time to adapt the instantaneous angle of attack, might explain discrepancies between obtained experimental results and predictions obtained by QS theory. A theoretical-analytical model based on the introduction of a time delay to the QS model is presented. As a result, in contrast to the classical QS model, the new formulation describes more accurately the amplitude of oscillations, predicts the phase delay angle and the frequency of oscillations, and provides a consistent QS applicability condition.

In terms of energy transfer in FIV, it has been observed that a cantilever with an attached prism, the most common configuration studied in the literature, usually obtains less power than predicted by classical TG formulation based on QS models. The instantaneous angle of attack of the cantilever configuration prism depends not only on classical galloping parameters but also on the rotation angle at the cantilever free end. A theoretical model was developed considering this effect and its negative impacts on the energy harvesting efficiency were delimited and 
discussed.

Additionally, a theoretical study is presented concerning power extraction via VIV of a circular cylinder in a dual-mass configuration. The dual mass system is modelled as a simplified two-degrees-of-freedom mechanical system where fluid forces on the circular cylinder are taken out of experimental data from forced vibration tests. Appropriate dual mass configurations can optimize power extraction in certain scenarios. System behaviour is characterized as a function of the governing parameters that could be used for designing purposes. Taking advantage of the developed two-way mass interaction formulation, the dual-mass equations were applied to a theoretical study of the coupling between a VIV cylindrical resonator and its associated linear electromagnetic generator, where the dominant masses are the stator and translator of the generator and a realistic milliwatt-energy harvesting device was designed and its performances were discussed. 


\section{Resumen}

Las Vibraciones Inducidas por el Flujo (FIV, por sus siglas en inglés) transversales de cuerpos flexibles de sección roma suponen un tema importante desde el punto de vista científico y práctico. En los últimos años, las FIV han despertado un gran interés tanto en la ingeniería civil, donde el objetivo es la mitigación de sus efectos, como en el campo de las energías renovables, donde se busca el aumento de la transferencia de energía. Estas aplicaciones aparentemente opuestas refuerzan la necesidad de una comprensión profunda de los fenómenos de las FIV, aplicada tanto al diseño como al estudio paramétrico de las FIV en estructuras y dispositivos de extracción de energía renovable.

Sin embargo, los modelos teóricos disponibles en la literatura tienden a ser incompletos y demasiado restrictivos para su aplicación en dispositivos de extracción de energía por las FIV. Esta Tesis pretende ampliar y profundizar los conocimientos en este campo a través de estudios fundamentales y de la investigación experimental de la fenomenología de las FIV y sus aplicaciones prácticas como fuente de energía renovable.

Para los cuerpos esbeltos de sección roma, existen dos tipos clásicos de FIV: Vibraciones Inducidas por Torbellinos (VIV, por sus siglas en inglés), donde el desprendimiento de los torbellinos está sincronizado con la frecuencia natural del cuerpo; y Galope Transversal (TG, por sus siglas en inglés), una inestabilidad inducida por el movimiento, descrita a menudo por modelos cuasi-estacionarios (QS) que, para ser aplicados, requieren de la hipótesis de oscilaciones lentas en comparación con el tiempo convectivo característico del flujo.

En una primera aproximación en el estudio del TG, se llevaron a cabo experimentos en un canal de agua recirculante de superficie libre, donde se tomaron medidas de las oscilaciones transversales inducidas por flujo en un prisma de sección cuadrada con baja relación de masas y amortiguamiento mecánico bajo. Los resultados experimentales no sólo ampliaron los datos publicados, sino que además permitieron obtener una nueva visión de los fenómenos, pues se obtuvo un nuevo parámetro adimensional, una 'verdadera' velocidad reducida. Este nuevo parámetro proporcionó, por primera vez en la literatura, un original gráfico de re-normalización de la amplitud de las oscilaciones. Este descubrimiento permite determinar la velocidad a la que se espera que las oscilaciones comiencen y cómo se correlacionan con una oscilación sinusoidal, así como establecer la validez de las condiciones QS.

Por otro lado, la hipótesis de que el flujo alrededor del cuerpo en el tiempo $t$ no tiene tiempo suficiente para adaptar el ángulo de ataque instantáneo, podría explicar las discrepancias entre los resultados experimentales obtenidos y las predicciones obtenidas por la teoría QS. Se presenta un modelo teórico-analítico basado en la introducción de un desfase en el tiempo en el modelo QS. Como resultado, y en contraste con el modelo QS clásico, la nueva formulación describe con mayor precisión la amplitud de las oscilaciones, predice el ángulo de desfase y la frecuencia de las oscilaciones, y proporciona una condición de aplicabilidad consistente de la hipótesis QS.

En cuanto a la transferencia de energía en FIV, se ha observado que un voladizo con 
un prisma solidario a un extremo, que es la configuración más comúnmente estudiada en la literatura, obtiene menos potencia de lo previsto por la formulación TG clásica basada en modelos QS. El ángulo instantáneo de ataque del prisma de la configuración en voladizo depende no sólo de los parámetros clásicos de Galope sino también del ángulo de rotación del extremo libre en voladizo. Se ha desarrollado un modelo teórico considerando este efecto y se han delimitado y discutido sus impactos negativos en la eficiencia de transferencia de energía.

Además, se presenta un estudio teórico para la extracción de energía a través de las VIV de un cilindro circular en una configuración de doble masa. El sistema de doble masa es modelado como un sistema mecánico simplificado de dos grados de libertad donde las fuerzas fluidas en el cilindro se toman de unos datos experimentales de vibración forzada. Se ha observado que las configuraciones de doble masa apropiadas pueden optimizar la extracción de energía en ciertos escenarios. El comportamiento del sistema se caracteriza en función de los parámetros gobernantes que podrían ser utilizados para propósitos de diseño. Aprovechando la formulación acoplada de interacción de masas desarrollada, las ecuaciones de doble masa se aplicaron a un estudio teórico del acoplamiento entre un resonador cilíndrico VIV y su generador electromagnético lineal asociado, donde las masas dominantes equivaldrían al estátor y al "rótor" lineal del generador. Por último, y a partir de todo lo anterior, se ha diseñado un dispositivo realista de recolección de energía del orden de milivatios y discutido varios aspectos teóricos en relación a su funcionamiento. 


\section{Publications associated to the Thesis}

- Xu-Xu J, Barrero-Gil A, Velazquez A 2015 A theoretical study of the coupling between a vortex-induced vibration cylindrical resonator and an electromagnetic energy harvester Smart Materials and Structures 24115009

- Xu-Xu J, Barrero-Gil A, Velazquez A 2016 Dual mass system for enhancing energy extraction from Vortex-Induced Vibrations of a circular cylinder International Journal of Marine Energy 16 250-261

- Xu-Xu J, Barrero-Gil A, Velazquez A 2016 Experimental study on transverse flow-induced oscillations of a square-section cylinder at low mass ratio and low damping Experimental Thermal and Fluid Science 74 286-295

- Xu-Xu J, Vicente-Ludlam D, Barrero-Gil A 2016 Theoretical study of the energy harvesting of a cantilever with attached prism under aeroelastic galloping European Journal of Mechanics B/Fluids 60 189-195 



\section{Contents}

$\begin{array}{ll}\text { Acknowledgement } & \text { i }\end{array}$

Abstract $\quad$ iii

Resumen $\quad$ V

Publications associated to the Thesis vii

1 Introduction $\quad 1$

1.1 Interest and aim of the Thesis . . . . . . . . . . . . . . . . . . 1

1.1 .1 Interest . . . . . . . . . . . . . . . . . 1

1.1.2 The aim of the Thesis . . . . . . . . . . . . . . 2

1.2 VIV and Transverse Galloping. Governing parameters. . . . . . . . . . . 3

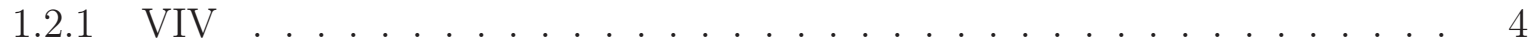

1.2.2 Transverse Galloping . . . . . . . . . . . . . . . . 8

1.3 A new field of application: VIV and Transverse Galloping for energy harvesting 11

1.4 Objectives, outline and main results of the Thesis . . . . . . . . . . . . 13

$\begin{array}{lll}2 & \text { Experimental study of a square prism } & 17\end{array}$

2.1 Introduction . . . . . . . . . . . . . . . . . . . . . 17

2.2 Experimental set-up and validation . . . . . . . . . . . . . . . . . 19

2.2.1 Experimental apparatus . . . . . . . . . . . . . . . . . . . . . . . . 19

2.2 .2 Data reduction . . . . . . . . . . . . . . . . . . 23

2.2 .3 Validation test . . . . . . . . . . . . . . . . . . . 24

2.3 Results . . . . . . . . . . . . . . . . . . . . . . 26

2.3 .1 Amplitude response . . . . . . . . . . . . . . . . . 26

2.3.2 Frequency response . . . . . . . . . . . . . . . . . . . . . . . . . . 29

2.3.3 Re-normalized amplitude response . . . . . . . . . . . . . . . . . . 30

2.3.4 Amplitude response in primitive variables . . . . . . . . . . . . . . . 32

2.3.5 Phase delay: Quasi-steady conditions . . . . . . . . . . . . . . . . . 34

2.4 Conclusions . . . . . . . . . . . . . . . . . . . . . . . . . . . . . 35

Chapter Appendix . . . . . . . . . . . . . . . . . . . 37

3 Quasi-steady time delayed model $\quad 39$

3.1 Introduction . . . . . . . . . . . . . . . . . . . . . . . . . . 39

3.2 Quasi-steady time delayed model . . . . . . . . . . . . . . . . . 40

3.2.1 Classical Quasi-steady modelling . . . . . . . . . . . . . . . . 41

3.2.2 Time delayed Quasi-steady modelling . . . . . . . . . . . . . . . . 42

3.3 Validation of the model . . . . . . . . . . . . . . . . . . . . 45 
3.3.1 Free vibration experiments. Analytical approach . . . . . . . . . . . 45

3.3.2 Forced vibrations of a square cylinder . . . . . . . . . . . . 51

3.4 Concluding remarks . . . . . . . . . . . . . . . . . . . . . 53

4 Energy harvesting of a cantilever-mounted prism $\quad 55$

4.1 Introduction . . . . . . . . . . . . . . . . . . . . . 55

4.2 Theoretical model . . . . . . . . . . . . . . . . . . . . 56

4.2.1 Aerodynamic force . . . . . . . . . . . . . . . . 57

4.2 .2 Piezoelectric force . . . . . . . . . . . . . . . . . . . . 59

4.2.3 Dimensionless equation . . . . . . . . . . . . . . . . . 60

4.2.4 Electrical power . . . . . . . . . . . . . . . . . . . 61

4.3 Analytical solution . . . . . . . . . . . . . . . . . . . . 61

4.3.1 Dynamic response . . . . . . . . . . . . . . . . . . 61

4.3.2 Electrical power . . . . . . . . . . . . . . . . . 63

4.4 Model validation . . . . . . . . . . . . . . . . . . . . . 65

4.5 Conclusions . . . . . . . . . . . . . . . . . . . . . 67

5 Dual mass system for enhancing energy extraction $\quad 71$

5.1 Introduction . . . . . . . . . . . . . . . . . . . 71

5.2 Mathematical model for VIV with dual mass system . . . . . . . . . . . . . . 72

5.2 .1 Mathematical model . . . . . . . . . . . . . . . . . 72

5.2 .2 Dual-mass VIV response . . . . . . . . . . . . . . . . . 75

5.2 .3 Energy harvesting efficiency . . . . . . . . . . . . . . . . 78

5.3 Dual-mass versus single-mass configuration . . . . . . . . . . . . . . . . . 79

5.3.1 Mechanical properties selection of the dual mass system . . . . . . . . . 81

5.4 Application example . . . . . . . . . . . . . . . . . . . . . 82

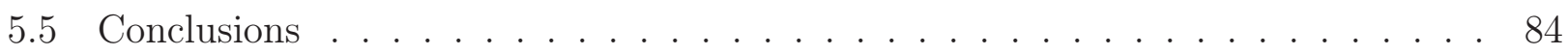

Chapter Nomenclature . . . . . . . . . . . . . . . . . . . 86

6 A VIV based electromagnetic energy harvester $\quad 89$

6.1 Introduction . . . . . . . . . . . . . . . . . . . . . . . 89

6.2 Description of the concept . . . . . . . . . . . . . . . . . . . . . 91

6.3 Mathematical model . . . . . . . . . . . . . . . . . . . . 92

6.4 Solution method . . . . . . . . . . . . . . . . . . . . . . . . 94

6.5 Application case . . . . . . . . . . . . . . . . . . . 96

6.6 Conclusions . . . . . . . . . . . . . . . . . . . . 102

7 Conclusions and future work $\quad 103$

7.1 Conclusions and resume of Chapters . . . . . . . . . . . . . . . 103

7.2 Future works . . . . . . . . . . . . . . . . . . 106

$\begin{array}{ll}\text { Bibliography } & 109\end{array}$ 


\section{List of Figures}

1.1 Scheme of a cantilever mounted prism. . . . . . . . . . . . . . . . .

1.2 Concept scheme of the studied problem (front view where the prism axis is perpendicular to the page). . . . . . . . . . . . . . . .

1.3 Visualization of the Kármán vortex street behind a cylinder; the flow is made visible through electrolytic precipitation at $R e=125$ (Barrero-Gil, 2008, PhD Thesis). . . . . . . . . . . . . . . . . . . 5

1.4 Experimental set-up in Cornell-ONR Water Channel (Khalak y Williamson, 1997). 6

1.5 Typical VIV (a) amplitude and (b) frequency responses for $m^{*}=2.4$ and $m^{*} \zeta=$ 0.013 (Khalak and Williamson, 1997).

1.6 (a) Fluid forces on a circular section in presence of a transverse flow. (b) Fluid forces on a D-section prone to gallop. The schemes on the left are for steady states and on the left are for motion states. . . . . . . . . . . . . . . . 9

1.7 Typical amplitude response for Transverse Galloping (Nemes et al. 2012). . . . . 10

2.1 Variation of $A^{\prime}$ with $U^{\prime}$.

2.2 Schematic of the free-surface water channel. Dimensions are given in mm. . . . . 20

2.3 Sketch of the experimental set-up (all dimensions are in millimeters). . . . . . . 21

2.4 Photograph of a close view of the elastic system. . . . . . . . . . . . . . . 22

2.5 Dimensionless velocity distribution (a) and turbulence intensity distribution (\%) (b) at the test section for a mean water velocity $\mathrm{U}$ of $0.36 \mathrm{~m} / \mathrm{s} \ldots \ldots . . .23$

2.6 Normalized amplitude variation with reduced velocity in VIV of a circular cylinder. 25

2.7 (a) Normalized amplitude variation with reduced velocity in VIV of a circular cylinder. (b) RMS total lift force coefficient variation with the reduced velocity from the same experiments. . . . . . . . . . . . . . . 26

2.8 Variation of the normalized amplitude $A^{*}(\mathrm{a}), H_{A}$ correlation coefficient (b), and normalized frequency of oscillations $f^{*}(\mathrm{c})$, with reduced velocity.

2.9 (a) Dependence of fluid force coefficient in phase with prism acceleration with reduced velocity for all tests. (b) $F=f^{*} / f_{C O M P}^{*}$ variation with reduced velocity from tests; $f^{*}$ is the measured reduced frequency, $f_{C O M P}^{*}$ is given by Eq. 2.6. . . 30

2.10 Normalized amplitude variation with true reduced velocity. . . . . . . . . . . 31

2.11 (a) Normalized amplitude response of several experiments $\left(A^{*}=A_{10}^{*}\right)$ as a function of true reduced velocity. (b) Normalized amplitude variation $\left(A^{*}=\right.$ $\left.\sqrt{2} A_{R M S}^{*}\right)$ with true reduced velocity. In both figures, present results are also

2.12 Variation with $m^{*}+m_{A}^{*}$ of the reduced velocity at which oscillations starts $U_{0}^{*}$.

2.13 Phase delay prediction from Eq. 2.13 and phase delay measurements in wind tunnel experiments by Bearman et al. (1987).

2.14 (a) Detail of the elastic system. (b) Natural frequency of oscillations variation with total mass. . . . . . . . . . . . . . . . . . . . . . . . . 
2.15 (a) Free decay oscillations for $m=3.72 \mathrm{Kg}$. (b) Frequency of oscillations (solid line) and damping ratio (dashed line) variation with the amplitude of oscillation. 38

3.1 Variation of $C_{Y}$ with tan $\alpha$ for square section from Bouclin and Parkinson (1977); for triangular and rectangular sections from Bokaian and Geeola (1983). . . . . .

3.2 Normalized steady-state amplitude of oscillations variation with reduced velocity in Nemes at al. (2012), white dots; and Bouclin and Parkinson (1977), filled squares.

$3.3 F$ variation with the reduced velocity and the mass ratio. $\zeta=0 \ldots \ldots 47$

3.4 Variation of $U_{0}^{*}$ with $m^{*} \ldots \ldots \ldots \ldots \ldots \ldots$

3.5 Phase angle from Bearman et al. (1987) experiments. $m^{*}=1013$ and $\zeta=0.0006$ (black circles) and $\zeta=0.0009$ (white circles). Solid is given by Eq. 3.26.

$3.6 A^{*}$ and $f^{*}$ variation with reduced velocity $U^{*}$. From left to the right: Nemes et al. (2012), Zhao et al. (2014) and Xu-Xu et al. (2016b). Model prediction in solid line.

$3.7 A^{*}$ and $f^{*}$ variation with reduced velocity $U^{*}$ for isosceles triangular and rectangular cross section. Model prediction in solid line. . . . . . . . . . . . .

3.8 Fluid force coefficient as a function of $\lambda^{*-1} \cdot A^{*}=0.675$ (exp: circles; model: dashed line) and $A^{*}=2$ (exp: squares; model: solid line).

4.1 (a) Typical arrangement piezoelectric energy harvesting from Galloping of a cantilevered prism. (b) One-degree-of-freedom model: vertical displacement of the rigid bluff body. . . . . . . . . . . . . . . . . . 56

$4.2 U_{\eta \max }^{*} / U_{g}^{*}$ dependence with $\delta U_{g}^{*} \ldots \ldots \ldots \ldots \ldots \ldots \ldots$

4.3 (a) Mean power variation with airspeed. (b) Efficiency dependence with airspeed. Open square stands for experimental results from Zhao et al. (2013) in the configuration where $m^{*}=101.7$ and $\delta=0.4 \ldots \ldots \ldots$. . . . . . .

4.4 (a) Mean power variation with airspeed. (b) Efficiency dependence with airspeed. Open square stands for experimental results from Zhao et al. (2013) in the configuration where $m^{*}=152.6$ and $\delta=0.4 \ldots \ldots \ldots \ldots$

4.5 (a) Contour plot of $A^{*}$ dependence with $U^{*}$ and $\delta$. Governing parameters correspond to those of Configuration 1. (b) Contour plot of $P_{E}$ dependence with $U^{*}$ and $\delta$. Governing parameters correspond to those of Configuration $1 . \ldots 69$

5.1 Sketch of the VIV of a circular cylinder in a dual mass configuration. . . . . . . 73

5.2 Contour plot of $c_{L v}$ (a) and $c_{L a}$ (b) in terms of dimensionless amplitude and the "true reduced velocity". . . . . . . . . . . . . . . 76

5.3 Maximum harnessable efficiency for a dual mass system where no dissipation is present $\left(\zeta_{1}=0\right)$ in terms of equivalent damping and mass parameters. . . . . . 80

5.4 Optimal $\zeta_{E}$ (electric damping) dependence with $\mu$ and $\Omega$ for (a) $\omega^{*}>1$ and (b) $\omega^{*}<1 . m^{*}=2, \zeta_{1}=0.01 \ldots \ldots \ldots \ldots \ldots \ldots$

5.5 Amplitude ratio $K^{*}$ contour plot over $\mu$ and $\Omega$ for (a) $\omega^{*}>1$ and (b) $\omega^{*}<1$.

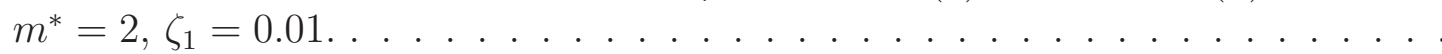


5.6 Detail of $K^{*}$ (a) and $\omega^{*}$ (b) dependence with $\mu$ and $\omega$ in the zone of interest

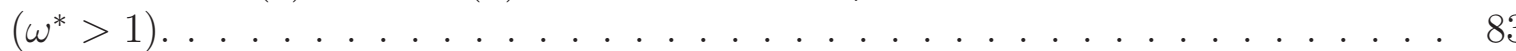

5.7 Efficiency as a function of the reduced velocity for the single mass configuration (a) and the dual mass configuration (b). . . . . . . . . . . . . . . 84

5.8 (a) Normalized amplitude of oscillations variation with the reduced velocity for the single mass configuration and (b) for the dual mass one. . . . . . . . . . . 85

6.1 Top view of the configuration being considered. . . . . . . . . . . . . . . 91

6.2 Sketch of a linear vibration driven electromagnetic generator. . . . . . . . . . . . 92

6.3 Sketch of a possible practical realization. . . . . . . . . . . . . . . 93

6.4 Model scheme. . . . . . . . . . . . . . . . . . . . . . . 94

6.5 (a) Output power and (b) conversion efficiency versus incoming flow velocity for the selected optimum design. . . . . . . . . . . . . . . . . . . . . . 98

6.6 (a) Maximum attainable efficiency as a function of $\zeta_{2}$ and $\Omega$; (b) expended plot of Figure 6.6a in the region where branching occurs. . . . . . . . . . . . . . . . . 99

6.7 Value of $U^{*}\left(U_{\max }^{*}\right)$ that maximizes efficiency as a function of $\zeta_{2}$ and $\Omega$. . . . 100

6.8 (a) Design curves $\lambda_{2}$ in terms of $\lambda_{1}$ for $m^{*}=2.5$ and $\mu=1$. (b) Design curves $\lambda_{3}$ in terms of $\lambda_{1}$ for $m^{*}=2.5$ and $\mu=1 \ldots \ldots \ldots 2 \ldots \ldots \ldots \ldots$ 



\section{List of Tables}

1.1 Quantitative data and results from studies and contributions made by some researchers in recent years. . . . . . . . . . . . . . . . . . 12

2.1 Mechanical data in the five configurations studied for the square-section prism. . 26

4.1 Physical properties in Zhao et al. (2013) experiments. . . . . . . . . . . . 66

6.1 Characteristics of some mini energy harvesters studied in the literature. . . . . . 97

6.2 Results of the multiobjective optimization $\left(c_{2}=0.217 \mathrm{Ns} / \mathrm{m}, k_{2}=195 \mathrm{~N} / \mathrm{m}\right) . .98$ 



\section{Chapter 1}

\section{Introduction}

\subsection{Interest and aim of the Thesis}

\subsubsection{Interest}

When a fluid flows around a solid body, it applies forces on it. If the solid is deformable, it will respond to these fluid forces either by adopting a stationary deformation or by continuously deforming itself over time, generally in an oscillatory manner. The Fluid-Structure Interaction will be more important as the structure response becomes more significant. In general terms, this may occur when at least one of the following conditions exists: (i) the shape of the solid body (in contact with the fluid flow) is appropriate, (ii) the average density of the solid is of the order of the density of the fluid, (iii) the reduced capacity of the structure to dissipate energy, (iv) very slender solid geometry, or (v) high dynamic pressure of the flow in relation to the elastic modulus of the solid. The question is that it is not uncommon that one (or several) of these conditions appeared in practical situations, and Fluid-Structure Interaction phenomena are relatively frequent, such as the interaction of the wind with trees, their leaves, or vegetation in general (De Langre, 2008), the locomotion of animals based on Fluid-Structure Interaction phenomena (birds, fish, insects), instability of flexible water pipes (hose), musical instruments or in many engineering applications, like in civil engineering: slender bridges, slender chimneys, high-rise buildings, marine structures (hauling cables, oil drilling and extraction machinery, mooring lines, piping, mooring structures, or in the aerospace field: flutter of aircraft surfaces, blades of turbo-machinery, wind turbine blades.

The above mentioned examples are indicative of the applied interest of the field and justify by themselves their detailed study, but it is also necessary to highlight the interest of Fluid-Structure Interaction from a fundamental perspective. They usually present a very rich phenomenology due to the complex interaction between the flow around the body (usually detached flow with large-scale vortex formation) and the elastic response of the body. The flow around the body (either stationary or in motion) provokes fluid forces on the body's surface. These fluid forces might modify the shape or the dynamics of the body (orientation, velocity), which successively changes the flow around the body. Thus, a strong coupling between the flow and the body in question appears, requiring an integral description of the problem. The number of parameters involved in the problem is usually not small since the problem is governed by the parameters describing the flow as well as the parameters describing the body's dynamics, and usually many parameters have to be taken into account, among others one could mention here the mass ratio (meaning density of the body over fluid density), mechanical dissipation of the body, the reduced velocity, the Reynolds number, roughness of the body, flow confinement, 
aspect ratio, etc. Non-linear resonances, hysteresis phenomena, lock-in, quasi-periodicity or other phenomena has been traditionally observed.

In addition, in the last decade, the interest has been even enlarged since some FlowInduced Vibration phenomena are being considered to extract useful energy (generation of electric currents) from the fluid flow. In these cases, the aim is to promote the Fluid-Structure Interaction phenomenon, in order to extract part of the kinetic energy from the flow and to transfer it to the body in form of mechanical oscillatory energy, and subsequently convert this mechanical energy into electrical energy by electromagnetic, piezoelectric, or electrostatic means. Bernitsas and his group of collaborators (Bernitsas et al. 2008) proposed in 2006 a system for electric power generation from marine/river currents taking advantage of the phenomenon of oscillations induced by vortex detachment in cylinders (VIV, acronym of VortexInduced Vibrations which is how the phenomenon is known in the specialized literature). Later on, in Barrero-Gil et al. (2010) the idea of taking advantage of the Transverse Galloping (TG) phenomenon was introduced and, through an analytical model, the roles of different main parameters that govern the problem were established and high energy transfer potential was proved. Since then, different concepts of energy extraction based on TG have appeared, focused on a significant production of electric energy (Barrero-Gil et al. EP2932091 B1, Barrero- Gil et al. US2015330358), or to generate small amounts of electrical energy (in the order of milliWatts) that can be used to get autonomous sensors and actuators and to avoid their dependence on batteries.

From the above, it follows that to study and to understand, as far as possible, FluidStructure Interaction problems have interest from a double perspective: (i) basic, because of their phenomenological richness and multidisciplinary character, and (ii) the applied side, since their study can help to better design structures against unwanted flow-structure phenomena or to propose methods to attenuate pernicious effects, as well as to take advantage of them to generate clean and sustainable energy by optimally designing Fluid-structure Interaction systems that maximize energy transfer from the flow.

\subsubsection{The aim of the Thesis}

In this Thesis two classical flow-structure problems are studied, from different perspectives. In first place, and with a basic or fundamental perspective, Transverse Galloping of square section prisms is studied, by hydro-elastic experiments in a free-surface recirculating water channel with the aim to understand more deeply this problem and try to shed light on several open questions, such as: is the amplitude of oscillations always linearly dependent on the reduced velocity as suggested previously by researchers? If so, which are the parameters governing this constant of proportionality?, Is there a reduced velocity value below which no oscillations appear? Is reduced velocity the most appropriate parameter to indicate whether the Quasi-steady hypothesis can be employed? In addition, a theoretical model based on the Quasi-steady model corrected by introducing a time delay is developed. It explains, at least qualitatively, experimental results and helps to answer the above-mentioned questions.

In the second part of the Thesis, with a more applied perspective, analytical models have been developed to predict and optimally design electrical generating devices taking advantage 
of the Transverse Galloping (TG) and Vortex-Induced Vibrations (VIV) phenomena. In the first case, the concept of small-scale energy generation is analysed (in the order of milli-watts) as recently proposed by Sirohi and Mahadik (2012). It consists in a rigid galloping prismatic body (rectangular, triangular, D-shaped, etc.) supported elastically by a cantilevered plate (see Fig. 1.1). At the base of the plate a piezoelectric sheet is employed for the generation of electrical current. In operation, once the unperturbed velocity of the incoming flow exceeds a threshold value, the prism begins to oscillate and induce a deformation in the piezoelectric sheet which generates an electric current that dissipates in an generic electrical impedance (in practical applications it can be, for example, a sensor). The cantilever assembly differs from the case of the classical Transverse Galloping because the instantaneous attitude of the body with respect to the incoming flow depends not only on the transverse velocity of the body's oscillation $(\dot{y})$ but also on the angle forming the end of the cantilevered plate with the horizontal $(\theta)$. As will be seen later in Chapter 4 , an analytical model has been developed, based on the Quasi-steady hypothesis that takes into account the effect of the aforementioned angle. It is seen how a new parameter appears in the problem that has great influence, which is the ratio between the characteristic transverse length of the body and the length of the cantilevered plate. In the second case, an analytical model is developed to predict the energy extraction from the VIV of a cylinder supported by springs, and it is studied if it is possible to improve the energy transfer by the use of a secondary mass elastically linked to the cylinder (by means of springs).

\section{(a)}

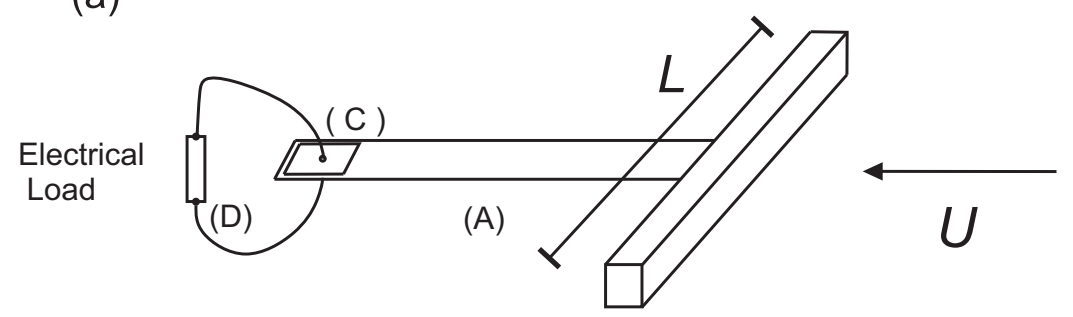

(B)

(b)

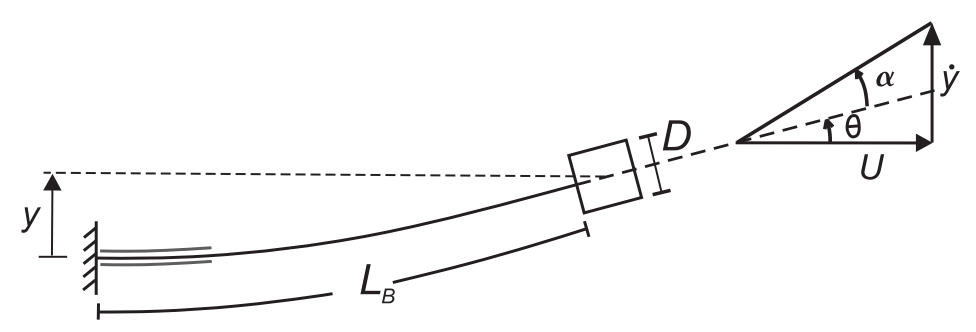

Figure 1.1: Scheme of a cantilever mounted prism.

\subsection{VIV and Transverse Galloping. Governing parameters.}

Within the large variety of Fluid-Structure Interaction phenomena that can be considered, in this Thesis two of them were studied, VIV and Transverse Galloping, which are probably the two paradigmatic Fluid-Structure Interaction problems when a bluff body (not streamlined) 
is subjected to the action of an external flow. The most realistic (and complex) situation is in which the elastic object is three-dimensional as well as the flow (three-dimensional and unsteady). However, since both VIV and Transverse Galloping usually take place in slender bluff bodies and most of time have a predominant oscillation direction (transverse to the incident flow), these facts make the most of experiments and numerical simulations consider the case of a rigid prismatic body supported elastically by springs which oscillates mainly in the transverse direction to the current (see Fig. 1.2).

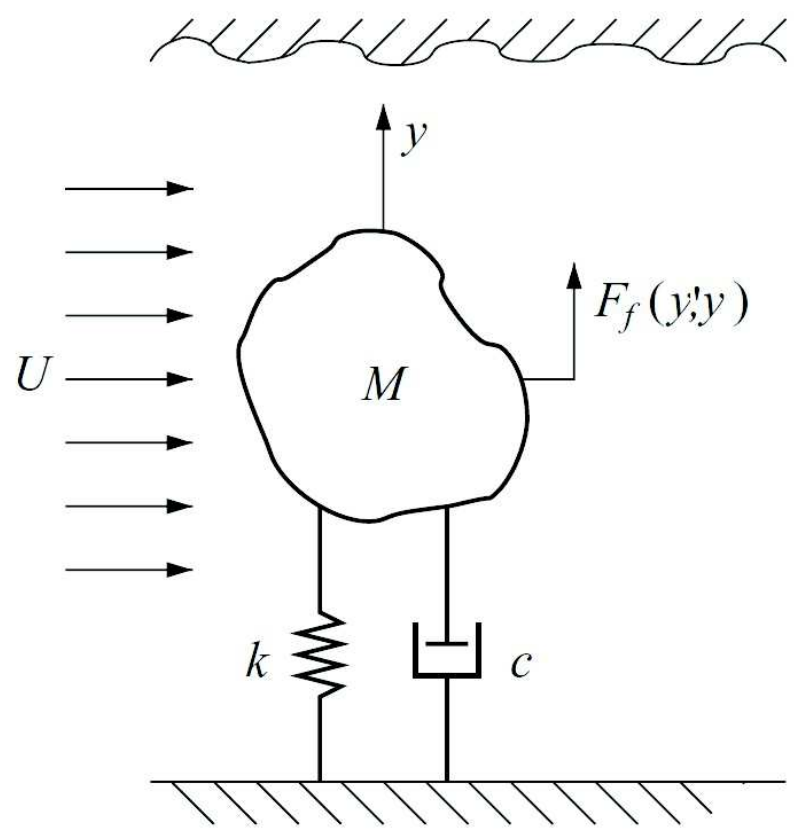

Figure 1.2: Concept scheme of the studied problem (front view where the prism axis is perpendicular to the page).

In the following there is a brief description of the VIV and Galloping phenomena. It may be said that the phenomenology of these problems is very varied, especially in the case of VIV, and this is not the purpose of this introductory chapter to be exhaustive. For more detail, the interested reader may wish to read the review articles of Parkinson (1989), Williamson and Govardham (2004) and Sarpkaya (2004).

\subsubsection{VIV}

The flow around a bluff body, from a certain number of Reynolds (of the order of 50 in the case of circular cylinders), provokes a periodic and alternating detachment of large-scale vortices, generating on the body surface an unsteady distribution of pressure that results in quasiperiodic fluctuating lift and drag forces. Fig. 1.3 shows the vortex street generated by a fixed cylinder for Reynolds number 125, visualized by the electrolytic precipitation technique. Regions of concentrated vorticity are shed alternately from both sides of the cylinder and convected to the wake with opposing directions of rotation. If the body is elastic it can respond to these forces giving oscillations, mainly in the transverse direction to the incident flow. These 
oscillations may become significant if the frequency of the lift force due to vortex shedding, proportional to the unperturbed flow speed, is close enough to the natural frequency of the elastic body. Once the oscillations begin, there is a complex interaction between the body motion and the fluid flow around it, so that under certain conditions the fluid flow around the object is amplified, organized and synchronized with the oscillation.
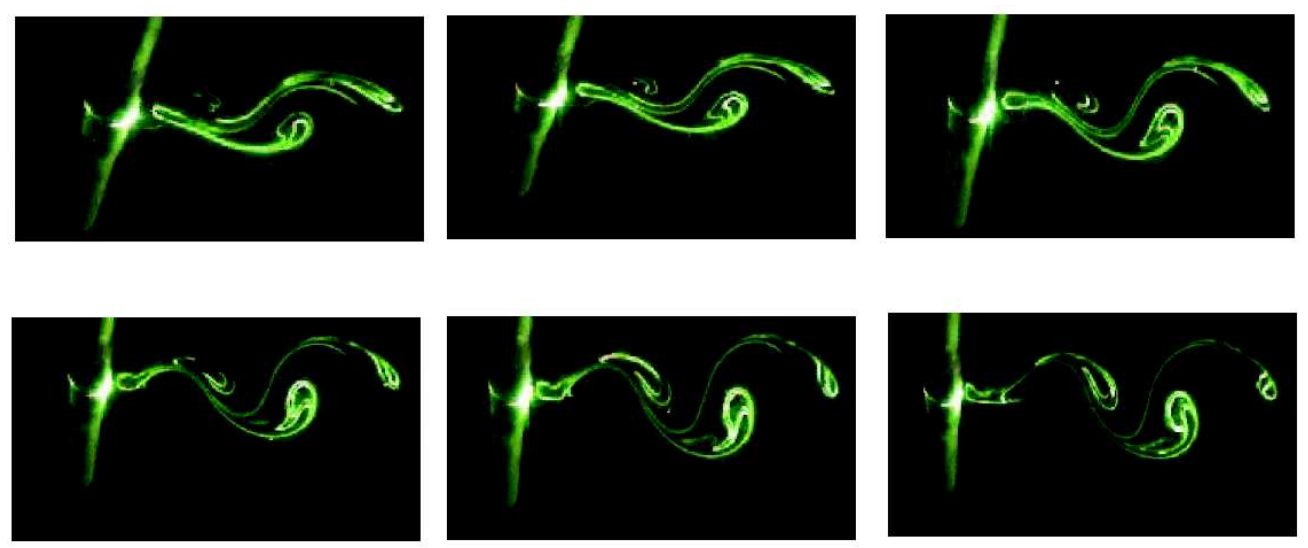

Figure 1.3: Visualization of the Kármán vortex street behind a cylinder; the flow is made visible through electrolytic precipitation at $R e=125$ (Barrero-Gil, 2008, PhD Thesis).

It may be instructive to describe the experiments of Khalak and Williamson (1997) in its hydrodynamic channel to explain some features of VIV. The experimental set-up employed is presented schematically in Fig. 1.4. It consists basically in a rigid cylinder of mass $m$ restricted to move solely along the transverse direction to the incident flow mounted on the rail of a linear guide. The rail of the guide is linked by springs (linear, constant stiffness $k / 2$ ) to fixed external points and the cylinder penetrates vertically into the water. When sliding the rail through the guide, there is very low friction (and almost viscous) because it incorporates air bearings. The motion of the prism is therefore only along the direction transverse to the incident flow $y$.

The equation of motion of the cylinder is based on the equilibrium between inertia force, damping force, stiffness and fluid force along the $y$-direction, $F_{Y}$ :

$$
m \ddot{y}+c \dot{y}+k y=F_{Y}=\frac{1}{2} \rho U^{2} D L C_{Y},
$$

where $m$ is the total mass in motion, $c$ is a mechanical damping constant, $k$ is the stiffness constant, $\rho$ is the fluid density, $U$ is the undisturbed velocity of the incident flow, $D$ the cylinder's diameter and $L$ its submerged length, and $C_{Y}$ is the instantaneous fluid force coefficient in the transverse direction to the incident flow. Finally, the dot symbol stands for differentiation with respect to physical time $t$.

Considering characteristic length $D$ and time $t^{*}=\omega_{N} t$, where $\omega_{N}$ is the circular frequency of oscillations in still water, the dimensionless equation for the dynamics of the body is found

$$
Y^{\prime \prime}+2 \zeta Y^{\prime}+Y=F_{Y}=\frac{U^{* 2}}{2 m^{*}} C_{Y}
$$




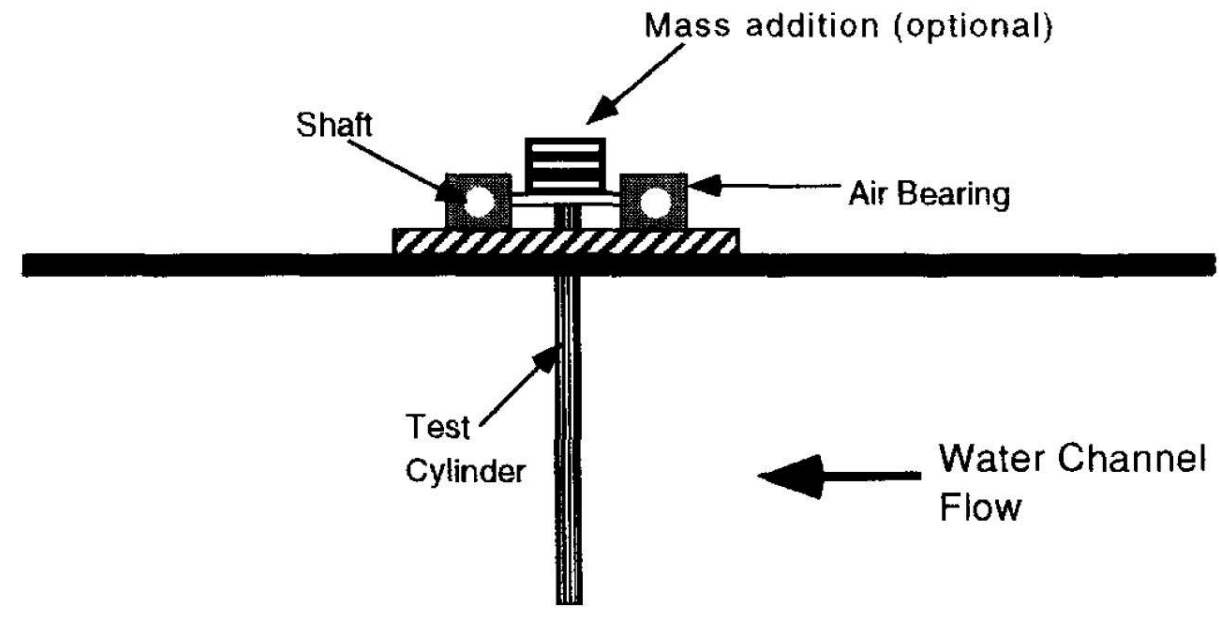

Figure 1.4: Experimental set-up in Cornell-ONR Water Channel (Khalak y Williamson, 1997).

where $Y=y / D, \zeta=c /\left(2 m \omega_{N}\right), U^{*}=U /\left(\omega_{N} D\right)$ is the reduced velocity which compares the characteristic oscillation time and the convective time, $m^{*}=m /\left(\rho D^{2} L\right)$ is the mass ratio, and the comma stands for differentiation with respect to the dimensionless time. The cylinder's response will then depend on the parameters $m^{*}, U^{*}$, and $\zeta$, as well as the Reynolds number. The response will be very different in those cases where $m^{*}$ is of order unity with respect to that in which $m^{*}$ is large. Fig. 1.5 shows a classic response in terms of steady-state amplitude and frequency of oscillations for a moderate value of mass ratio.
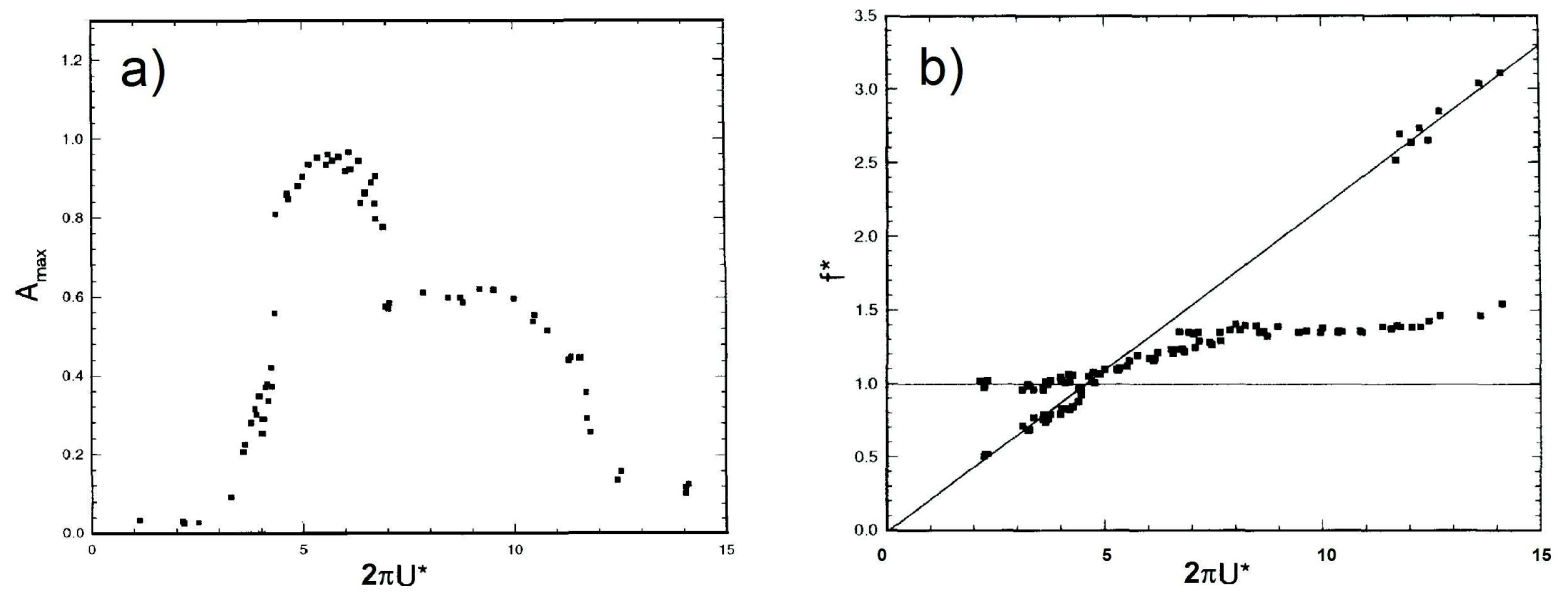

Figure 1.5: Typical VIV (a) amplitude and (b) frequency responses for $m^{*}=2.4$ and $m^{*} \zeta=$ 0.013 (Khalak and Williamson, 1997).

In sight of Fig. 1.5, it can be deduced:

- There is no significant oscillations until a value of the reduced velocity close to $4 / 2 \pi$. The normalized frequency of oscillations $f^{*}=f / f_{N}\left(f_{N}=\omega_{N} / 2 \pi\right)$ indicates that these 
initial oscillations has two different leading frequencies, close to the frequency given by the Strouhal law $\left(f^{*}=S t U / D f_{N}\right.$, where $S t \approx 0.2$ for a circular cylinder $)$ and the natural frequency.

- When $2 \pi U^{*} \approx 4.5$ an abrupt change in amplitude of oscillations is observed. This change is due to a transition in the wake pattern from a $2 S(C)$ mode to a $2 S$ mode.

- When $2 \pi U^{*} \approx 5$ (inverse of the Strouhal number of the cylinder), $f^{*} \approx 1$ and grows slowly with the reduced velocity until $U^{*} \approx 8$. In this range the mode of vortex shedding goes from $2 S$ (one single vortex shed per side of the oscillating cylinder) to $2 P_{0}$ (a pair of vortices shed per side of the oscillating cylinder, one of them more energetic than the other) and the amplitude of oscillations grows, reaches a maximum value, and diminishes. The shedding of vortices is synchronized with the cylinder's motion (lock-in). The competition between modes of vortex shedding can give place to an intermittent phenomena (the detachment changes from one mode to another without establishing a clear mode of detachment) or hysteresis (the amplitude of oscillation depends on the direction in which the frequency of excitation approaches to the natural frequency).

- In the range of $8<2 \pi U^{*}<11$ the amplitude of oscillation takes a quite constant value as well as the frequency of oscillations. The mode of vortex shedding is $2 P$ (a pair of vortices shed per side of the oscillating cylinder). The shedding continues synchronized with the cylinder's motion. An heuristic explanation to this lock-in is that the oscillation causes the distance between the shear layers where vorticity is generated to increase, the current velocity also increases and there is an apparent increase of the cylinder diameter due to the oscillation which is compensated with the increase of the flow speed, maintaining the frequency of vortex shedding approximately constant. The range of reduced velocities where lock-in takes place depends strongly on $m^{*}$ being larger for lower values of $m^{*}$.

- For $2 \pi U^{*}>11$ synchronization of vortex shedding and cylinder's oscillations is lost (lock-out), i.e. the vortices are shed without any link to the cylinder's position, and the amplitude of oscillation drops abruptly.

The amplitude response shows a somewhat bell-shaped character, which resembles a resonance character. However, this resonance is more complex than the traditional one observed in a linear oscillator under a forcing term due to its non-linear character. The range of the lock-in regime (where oscillations are significant) is highly influenced by the reduced mass (or mass ratio), being larger when the reduced mass is lower. In fact, for a cylinder, when the reduced mass $m^{*}$ is lower than a certain threshold (known as critical mass), the resonance between the wake and the elastic system occurs for all values of the fluid velocity and both frequencies are locked-in, thus large amplitude oscillations regime is expected for all values of the incident velocity (Govardhan and Williamson, 2002). Finally, it should be commented that the phenomenon is self-limited, meaning that the amplitude of oscillations is limited to the order of one diameter.

The approximations employed to study the VIV problem are mainly experimental or numerical, since flow separation makes impossible to obtain any self-enclosed analytic model from the fluid (Navier-Stokes) and the elasticity (Navier) equations. From an analytic perspective, there exists some heuristic and semi-empirical formulations where experimental information 
is introduced into the formulation in an "ad-hoc" way, through some parameters. Its validity is usually very limited and its predictive capacity low. For example, several wake-oscillator models has been proposed, where an ordinary differential equation for $C_{Y}$ is coupled with the cylinder dynamics equation (Facchinetti et al. 2004, Violette et al. 2007).

VIV has a great deal of practical importance, for example, it can cause vibrations in the tubes of heat exchangers; it is of importance in the design of slender bridges, buildings or marine structures; it influences the dynamics of pipelines that suck oil from the seabed; it can cause long vibrations in structures anchored by cables at sea; it can be as the means for a fluid flow mixing promoter (Sanchez-Sanz and Vazquez, 2009), and so on.

\subsubsection{Transverse Galloping}

Galloping is a flow-structure instability phenomenon of self-excited character, unlike VIV, in a sense that the excitation force of the motion disappears if the oscillations cease. It is commonly accepted that there are two kinds of Galloping: Transverse and Torsional. Nevertheless, in this Thesis, it will be restricted to the case of Transverse Galloping, where the motion of the body occurs mainly in the transverse direction to the incident flow (as in the case of VIV). It is relatively frequent to observe Galloping phenomena in electrical power lines, particularly in cold areas with high wind intensity where snow adheres to the cable and generates an unfavourable aerodynamic shape (typically D-shaped) that induces Galloping. It also appears in structures with low damping and certain cross-section geometry (typically rectangular, triangular or Dshaped cross-sections), or in circular pipes in marine environments where deposition of organic material generates a more or less elliptical or D-shaped geometry (Simpson, 1972).

The paradigmatic example is the case of a rigid prism with fixed cross-section shape (typically square, triangular, or D-shaped) supported by springs, which is subjected to the action of an incoming flow. Once a certain flow velocity is exceed, called Critical Galloping Velocity, the stabilizing effect of the structural damping is overcome by the destabilizing effect of the fluid force generated by a small transverse displacement of the prism. Then, an energy transfer of the flow to the prism appears and it begins to oscillate transversely to the current in a quasi-sinusoidal way with increasing amplitude until a situation of steady oscillations is reached quickly. The amplitude and frequency of oscillations in steady state depend mainly on the parameters that characterize the problem: straight section shape, prism mass $\left(m^{*}\right)$, mechanical damping $(\zeta)$, and incoming flow velocity $\left(U^{*}\right)$; note that as the motion is transverse to the incident flow, Eq. 1.1 posed to describe VIV is still valid, but the functional dependency of $C_{Y}$ will be different. Transverse Galloping does not appear in cylinders. It consists of a self-induced motion "controlled" by the induced angle of attack and appears in those geometries in which a variation of the induced angle of attack produces transverse fluid force in the direction of the oscillation velocity. Fig. 1.6 shows an illustrative example of a circular cylinder case (which does not gallop) and of a geometry that does gallop.

The most-likely to gallop cross-sections are, in general, those in which:

- Asymmetry appears in the cross-section. 

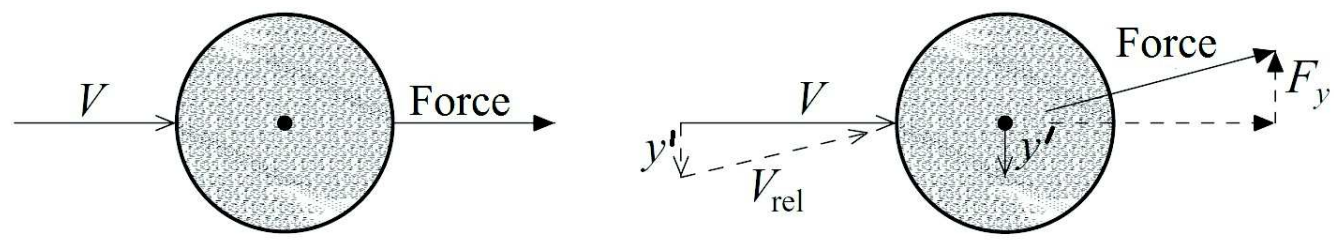

(a)
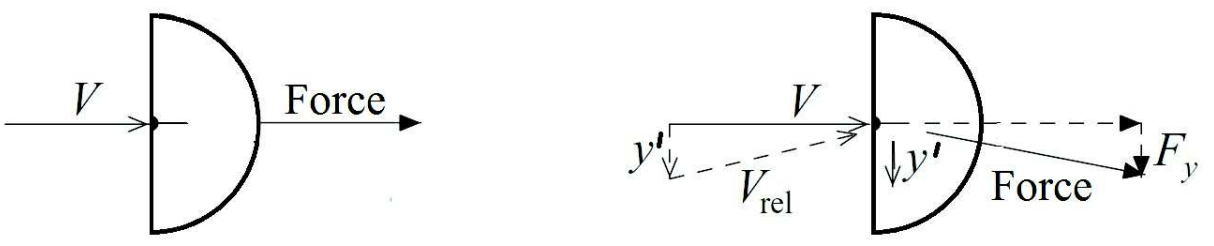

(b)

Figure 1.6: (a) Fluid forces on a circular section in presence of a transverse flow. (b) Fluid forces on a D-section prone to gallop. The schemes on the left are for steady states and on the left are for motion states.

- The existence of a short aft-body (part of the body which is downstream of detachment points).

- Minimal geometric area ahead of the aft-body: the upstream flow with respect to the detachment points is non-detached what has stabilizing effect. A representative case of this phenomenon is the configuration of a square section at angle of attack $45^{\circ}$ (configuration in diamond) that hardly suffers Galloping.

- There is no re-adherence of the flow in the aft-body.

Fig. 1.7 shows the variation of the amplitude of oscillations with respect to the reduced velocity in the case of Transverse Galloping of a square prism. As can be seen, from a given value of reduced velocity, the instability is triggered and the amplitude grows with the reduced velocity unlimitedly, since the instability is not due to a resonance effect along with evolution of vortices (as it was in the VIV case).

For high values of reduced velocity, the characteristic oscillation time (of order $f_{N}^{-1}$ ) is large compared to the convective time of flow $D / U$, so a possible analytical approach to characterize the fluid force coefficient $C_{Y}$ (see Eq. 1.2) is to resort to the Quasi-steady hypothesis. In this case, the instantaneous fluid force is assumed to be equivalent to the static fluid force on the object. The current approach of the motion-induced angle of attack is described in Den Hartog (1932), Parkinson and Brooks (1961), Barrero-Gil et al. (2009a). From Fig. 1.7, it follows that the motion-induced angle of attack $\alpha$ is given by

$$
\alpha=\tan ^{-1} \frac{-\dot{y}}{U}
$$




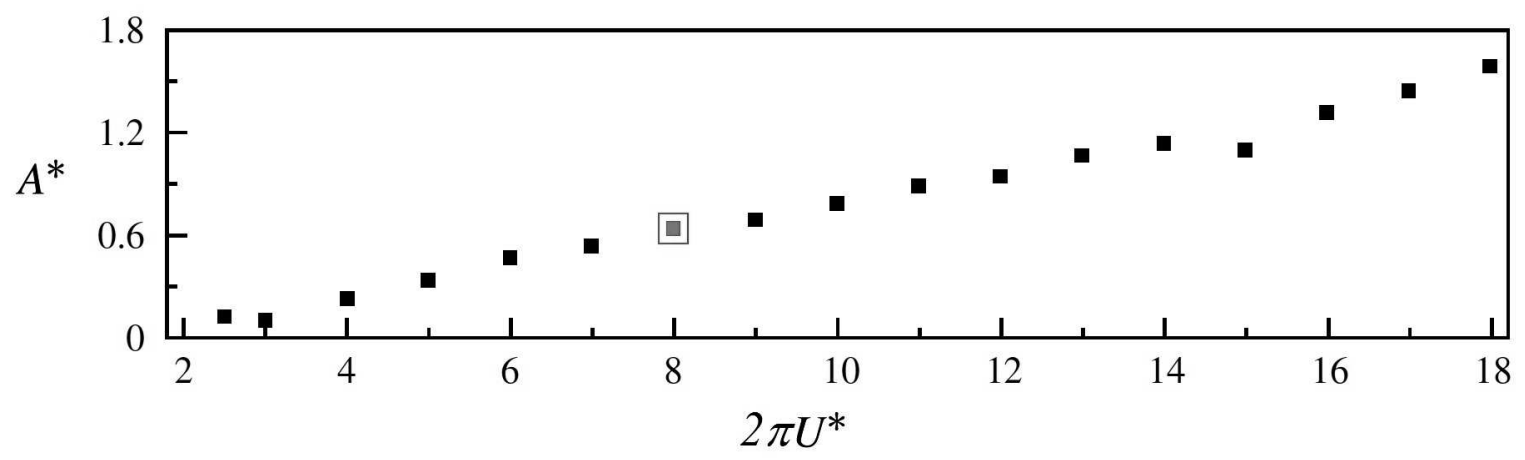

Figure 1.7: Typical amplitude response for Transverse Galloping (Nemes et al. 2012).

On the other hand the functional dependence of $C_{Y}$ with $\alpha$ can be obtained through static experiments (for example in wind tunnel) or in numerical simulations, and approximated by a polynomial relationship in terms of $\tan (\alpha)$ (Blevins, 1990),

$$
C_{Y}(\alpha)=\sum_{j} a_{j} \tan (\alpha)^{j}
$$

with $j=1,2,3, \ldots$

$C_{Y}$ may be written as

$$
C_{Y}(\alpha)=\sum_{j} a_{j}\left(\frac{\dot{y}}{U}\right)^{j},
$$

and taking into account the Eq. 1.5, the dynamics of the galloping body is described by the dimensionless ODE:

$$
Y^{\prime \prime}+2 \zeta Y^{\prime}+Y=F_{Y}=\frac{U^{* 2}}{2 m^{*}} \sum_{j} a_{j}\left(\frac{2 \pi Y^{\prime}}{U^{*}}\right)^{j} .
$$

From this equation, it can be seen that Galloping will appear in those cross-sections where $a_{1}$ is positive. In addition, it may be deduced that the critical reduced velocity at which Galloping starts is given by

$$
U_{G}^{*}=\frac{4 m^{*} \zeta}{a_{1}}
$$




\subsection{A new field of application: VIV and Transverse Gal- loping for energy harvesting}

A common design objective of elastic systems is to avoid the appearance of Fluid-Structure Interaction phenomena or, at least, to minimize its effects. However, in recent years, in some academic and technological fields, an opposing perspective has emerged. It consists of the enhancement of Fluid-Structure Interaction effects, having in mind the idea of extracting part of the kinetic energy of the incident flow and turning it into a more useful energy form (e.g. electricity). The basic idea is to convert part of the kinetic energy of the incoming flow into oscillatory mechanical energy through Fluid-Structure Interaction phenomena such as VIV or TG. Later, this mechanical energy is converted into electrical energy by the use of electromagnetic, piezoelectric or electrostatic transducers. Bernitsas et al. (2008) proposed the application of VIV of a circular cylinder to convert the hydrokinetic energy of the currents of rivers and oceans into electrical energy. VIV potential for energy extraction has also been studied by Grouthier et al. (2013), Mackowski and Williamson (2013), Barrero-Gil et al. (2012) or Sanchez-Sanz et al. (2009). It is also worth mentioning the STINGRAY project (Zhu and Peng, 2009), which consists of taking advantage of the coupled-mode flutter phenomenon in aerodynamic rigid profiles that can oscillate with two degrees of freedom (transverse to incident flow and rotational). Similar concept from Kinsey and Dumas (2008) should be cited. In 2010, Barrero-Gil et al. proposed for the first time in a theoretical way the possibility of using Transverse Galloping, of greater energy transfer and greater speed range than VIV, as a method of extracting renewable energy.

All of the above mentioned concepts are focused on the production of large scale useful energy, in which electromagnetic conversion is considered to transform mechanical energy into electrical energy. However, a very promising field of application is on the micro-scale applications, with levels of electrical generation in order of mili-Watts. In these cases, the mechanical energy to electric energy conversion may be done by piezoelectric means. Piezoelectrics are dielectric materials that can convert a mechanical deformation into potential difference and electrical charge. They are characterized by having a low cost of manufacture, small size, being mechanically resistant and chemically inert. In Anton and Sodano (2007) a review of these materials and their application to the field of energy generation is presented. Allen and Smith (2001) pioneered the use of piezoelectric sheets in the wake of a bluff cylinder. When the mass and elastic properties of the membrane are appropriately chosen, the large-scale vortex street formed behind the body induces significant oscillations in the membrane that can be converted into electricity. After Galloping was proposed by Barrero-Gil et al. (2010) as an efficient way to harvest fluid flow energy, the schematically shown concept has been proposed and investigated in detail theoretically and experimentally. From Fig. 1.1, a slender plate (A) is mounted in cantilever, and at its free end a rigid prismatic body (B) with cross-section prone to gallop (square, triangular, semicircular, etc.) is fixed. A sheet of piezoelectric material (C) is pasted to the base of the plate. When the velocity of the incident flow, whether air or water, exceeds the Critical Galloping Velocity, the prism begins to oscillate and induces deformations in the piezoelectric sheet and, consequently, electric current can be dissipated at an electric resistance (D) (note that in an actual application this electrical impedance could be a sensor). The advantages of this cantilever assembly is clear in terms of simplicity of assembly and low 
cost. In addition the use of a piezoelectric converter also brings advantages in terms of ease of manufacture, handling, cost, and durability. Much research has been done on the concept shown in Fig. 1.1, which gives an idea of the interest raised. Table 1.1 shows some studies and contributions made by some researchers in recent years.

Table 1.1: Quantitative data and results from studies and contributions made by some researchers in recent years.

\begin{tabular}{lllll}
\hline Author & $\begin{array}{l}\text { Experimental } \\
\text { Numerical }\end{array}$ & $\begin{array}{l}\text { Flow speed } \\
(\mathrm{m} / \mathrm{s})\end{array}$ & $\begin{array}{l}\text { Power } \\
(\mathrm{mW})\end{array}$ & $\begin{array}{l}\text { Device vol- } \\
\text { ume }\left(\mathrm{cm}^{3}\right)\end{array}$ \\
\hline Wu (2013) & Numerical & 10 & 2000 & 3340 \\
Abdelkefi (2013a) & Numerical & 5 & 10 & 22 \\
Amini (2014) & Numerical & 7.23 & 4 & 35 \\
Weinstein (2012) & Experimental & 2.5 & 0.2 & 620 \\
Jung (2011) & Experimental & 4.5 & 370 & 3340 \\
Akaydin (2010) & Experimental & 7.3 & 0.004 & 862026 \\
Akaydin (2012) & Experimental & 1.2 & 0.1 & 4237 \\
Sirohi (2011) & Experimental & 2.3 & 50 & 8695 \\
Zhao (2013) & Experimental & 2.5 & 8.4 & 240000 \\
Bibo (2015) & Numerical & 2.3 & 0.22 & 262193 \\
Dai (2015) & Numerical & 6 & 1 & 14130 \\
Tang (2015) & Experimental & 7 & 7 & 240000 \\
Ali(2013) & Experimental & 3.25 & 0.0375 & 625000 \\
\hline
\end{tabular}

In the aforementioned application, Fluid-Structure Interaction with energy harvesting purposes has a double coupling character (fluid-solid-electric). The range of governing parameters increases with respect to the case of fluid-solid coupling since new parameters associated to the solid-electrical coupling appear. Broadly speaking, electrical part introduces damping and stiffness effects into the dynamics of the body which will change the fluid-solid interaction, in a way that it is mandatory to include this effect and to perform a full modelling (fluid-solid-electric). This has not been much done up to date. Traditionally Fluid-Structure Interactions were undesired and generally considered dangerous, since unwanted oscillations might provoke from short-term destruction of the structure up to long-term wear problems like fatigue. Therefore, in the past, most of the research on fluid-solid instabilities dealt with the pernicious effects of these oscillations on the structures and were motivated by the identification of the phenomenon in order to avoid or control its presence. Thus, most of the existing literature in the past on such fluid-solid interactions emphasized on linear analysis as the interest was on determining when would such oscillations appear and on minimizing the amplitude of oscillations (and the energy transfer from the flow to the body). Also, much of the previous research dealt with weakly damped systems as it is a usual characteristic of civil engineering structures. On the other hand, energy harvesting Fluid-Structure Interactions looks for maximizing the energy transfer from the flow to the body and with situations of high damping with high amplitude level of oscillations. This requires a non-linear analysis of importantly damped bodies where amplitude of oscillation ought to be enhanced and promoted. While the fundamental notions responsible for the vibrations are well known, non-linear dynamics and their susceptibility to involved parameters, including the energy extraction process, remains in many cases to be bet- 
ter investigated. To properly design energy harvesting devices, like the one depicted in Fig. 1.1, there is several questions that are not fully answered, like:

- Which strategy should be followed to maximize electrical production from the fluid, solid, and electrical part point of view?

- How do the characteristics of the electrical part (impedance) influence the energy transfer efficiency. Is there an optimal impedance (which maximizes energy extraction)? If so, which is its dependence?

- In the cantilever assembly, as shown in Fig. 1.1, the situation is not completely analogous to that of the pure (classic) Transverse Galloping, since a motion of the prism appears in the direction of the flow (direction $\mathrm{x}$, in-line motion) in addition to the transverse direction. How does this in-line motion affect the dynamics of the prism and its potential to extract energy from the current?

\subsection{Objectives, outline and main results of the Thesis}

This Thesis has two different parts. The first one has a basic character and its main objective is to gain insight into the Transverse Flow-induced Oscillations of a square-section prism. The study of transverse Flow-induced Oscillations of a square-section has been traditionally focused on situations where the mass ratio $m^{*}$ is large, that is typically the case when airstream is considered. Under such circumstances, a successful theoretical approach to the problem is possible by resorting to the Quasi-steady hypothesis (Parkinson and Smith 1964, Barrero-Gil et al. 2009, 2010). However, studies where $m^{*}$ is of order of unity are scarce even though they may take place in practical situations (say, structural members in water flows). Only three experimental campaigns exists in the literature focused on the regime of $m^{*} \sim 1$. In this sense, an experimental campaign at the Antonio Barrero Ripoll free surface re-circulating water channel has been carried out which enlarges existing experimental results published in the literature. In addition, an analytical model, which explains results found experimentally, is developed and presented in Chapter 3. More specifically:

- Chapter 2 presents experimental results concerning the Transverse Flow-induced Oscillations of a square-section prism for several values of mass ratio, ranging from 2.2 to 14.4, and low mechanical damping. Experiments were carried out in a re-circulated free-surface water channel. A rigid square-section prism was fixed to an elastic system made with dual (parallel) metallic blades connected by a rigid block. By this way the square-section prism is free to oscillate in the transverse (normal to flow) direction with low damping. The elastic system presents linear behaviour in terms of stiffness and damping. The functional dependence between the steady-state amplitude and frequency of oscillations with reduced velocity is characterized. As a novelty it is found that a re-normalization plot appears when the steady-state amplitude of oscillations is plotted against the "true" reduced velocity, which is defined as the reduced velocity divided by the dimensionless frequency 
of oscillations. Experiments also allow to discuss other questions, like the reduced velocity at which oscillations are expected to start depends on the value of the mass ratio, how close to sinusoidal (say, fixed amplitude and frequency) the oscillations are, or when Quasi-steady conditions are expected to be met. Part of the content of this Chapter has been published in the Q1 Journal of Experimental Thermal and Fluid Science.

- In Chapter 3, a novel time delayed Quasi-steady theoretical model of Transverse Flowinduced Oscillations of a square-section prism is developed. The theoretical model is based on the introduction of a time delay which is only function of the static empirical coefficients used in the traditional Quasi-steady model. Previous experimental results (both in free and forced vibrations) agrees well with those given by the theoretical model. In addition, the model helps to gain insight about experimental results not explained satisfactory by the Quasi-steady theory. Part of this Chapter has been sent for publication.

The second part of the Thesis is devoted to gain insight into the potential of Transverse Galloping to extract efficiently energy from an airflow by piezoelectric means. Also, an improvement for the energy extraction from VIV is studied. It consists on introducing a secondary mass (dual-mass) to the system.

An improvement in the efficiency of energy harvesting with respect to that of the single mass configuration is found when the mechanical properties of the dual-mass system are appropriately chosen and certain design scenarios are given. In addition, dual-mass system promotes a broadening of the values of the reduced incident flow velocities at which the efficiency is kept high.

- In Chapter 4, the aeroelastic Galloping of a cantilever with attached prism is studied. This arrangement is not entirely analogous to that of classical Transverse Galloping since the instantaneous attitude of the galloping body (prism) with respect to the incident flow depends not only on the velocity of the galloping body and wind speed (like in classical Transverse Galloping) but also on the rotation angle at the cantilever free end. A new governing parameter emerges, namely the ratio of the cross-section length of the prism to the beam length $\delta$, and its effect on the galloping dynamics and power output needs to be studied. To this end, a theoretical model is here developed where the influence of $\delta$ is considered. Part of the content of this Chapter has been published in the Q2 European Journal of Mechanics B/Fluids.

- Chapter 5 presents a theoretical study concerning power extraction via Vortex-Induced Vibrations of a cylinder in dual-mass configuration. The dual mass system is modelled as a simplified two-degrees-of-freedom mechanical system where fluid forces on the cylinder are taken out of experimental data from forced vibration tests. It is shown that power extraction can be optimized in certain reconfiguration scenarios if dual mass parameters (i.e.: secondary mass and stiffness between masses) are chosen appropriately. More specifically, system behaviour is characterized as a function of the governing parameters, and performance charts are presented that could be used for parameter selection purposes in an engineering environment. Limitations and benefits of the redesigning with respect to the absence of the dual-mass are also presented. Part of the content of this Chapter has been published in the Q1 International Journal of Marine Energy. 
- In Chapter 6, taking advantage of the two-way mass interaction formulation detailed in the Chapter 5, the dual-mass equations have been applied to a theoretical study of the coupling between a VIV cylindrical resonator and a linear electromagnetic generator. The dominant masses of the mentioned formulation are the stator and translator of the generator. In addition, a realistic milli-Watt-energy harvesting device has been designed and its performances discussed. The rationale to carry out the study is the fact that in these types of configurations there is a two-way interaction between the moving parts in such a way that their motions influence each other simultaneously, thereby affecting the actual power harvested. It is believed that a theoretical model can shed some light on the nature of the interaction and, at the same time, provide design laws that can be used for practical design and optimization purposes, before resorting to complementary numerical simulations. Part of the content of this Chapter has been published in the Q1 Journal Smart Materials and Structures.

- Finally, in Chapter 7 some concluding remarks, open questions and recommendations for future works are briefly discussed.

Through the dissertation, each chapter forms a self-contained study, and therefore includes its own Introduction Section and Conclusions Section. However, it has been considered convenient to place all the references together at the end of the dissertation. 



\section{Chapter 2}

\section{Experimental study of a square prism}

\section{$2.1 \quad$ Introduction}

Flow induced vibration (FIV) of bluff bodies is a topic of interest from both scientific and engineering sides. In engineering applications, most structures have a bluff cross-section and, most of the time, they are under the action of wind or water flows which makes mandatory to estimate properly the fluid loading on the structure and its response. When considering scientific aspects, the problem is very rich: the bluff body under fluid flow sheds, for high enough Reynolds numbers, large-scale vortices which create an unsteady fluid loading on the body surface which can induce vibrations to the body. Then, there is a very complex interaction between the incoming flow and body response, which depends mainly on the shape of the body, structural (mechanical) properties of the body, and flow speed. The cylinder (circular transverse section) has been the bluff geometry which has been studied more intensively to date, because circular beams are common structural elements but also because of its inherent symmetry. A single isolated cylinder can be excited mainly by Vortex-Induced Vibrations (VIV) but also by buffeting if turbulence in the inflow is high enough. Two circular cylinders arranged in tandem can be excited by Wake-Induced Vibrations (WIV). Reviews on VIV of circular cylinders are given by Sarpkaya (2004) and Williamson and Govardham (2004); for a detailed introduction to WIV the reader is referred to Sumner (2010) or Assi et al. (2010, 2013).

Non-axisymmetric cross-sections can be excited by VIV as well as other FIV phenomena, like galloping (Naudascher and Rockwell, 2005). Galloping is a motion-induced instability that appears in some elastic bluff bodies (square cross-section at a zero-angle of attack for example) when the velocity of the flow exceeds a certain critical value. Then, a small transverse displacement of the body induces an angle of attack relative to the incoming flow and an asymmetric pressure distribution, so that fluid force appears in the direction of the displacement in such a way that energy is transferred from the current to the body and oscillatory motion (mainly transverse to the unperturbed flow) develops. Unlike VIV, which occurs only in a certain range of flow velocities and with self-limited amplitude, galloping takes place for any value of the flow velocity higher than the critical value and has a monotonic increase of amplitude with flow velocity.

The study of galloping has been traditionally focused on situations where the mass ratio $m^{*}$, the ratio of mean body density to fluid density, is large, that is the case when airstreams are considered $\left(m^{*} \gtrsim 50\right.$, for instance, for a light material 50 times the density of the air). Under such circumstances, a successful theoretical approach to the problem is possible by resorting to the Quasi-steady hypothesis (Parkinson and Smith 1964, Barrero-Gil et al. 2009, 2010). Quasi-steady hypothesis assumes that fluid forces are determined by the instantaneous attitude 
of the body (angle of attack) with respect to the incoming flow, and it may be expressed as a polynomial of the velocity of body oscillations (Blevins, 1990). This means that fluid force is in phase with the velocity of body oscillations at any instant and, therefore, the phase lag between fluid force and body displacement is $90^{\circ}$ (Parkinson, 1989). It is traditionally considered that Quasi-steady conditions can be resorted to when the characteristic convective time of the flow is small compared with the characteristic time of oscillations. However, studies on galloping for situations at which $m^{*} \sim 1$ (say hydroelastic) are scarce even though they occur in many practical situations (think, for example, in the case of prismatic structural members in water currents, Assi et al. 2015).

As far as it is known, it was Bouclin (1977), in collaboration with Parkinson, who were first interested in galloping features of a square-section when $m^{*} \sim 1$. They performed hydro-elastic experiments with a spring-mounted square-section prism of side length $D$ and submerged length $L$ in a free-surface recirculating water channel for three different values of $m^{*}(4.2,8.5$, and 14) and low structural damping. The square-section prism was restricted to move transverse to the incoming flow direction. Their results were given in terms of normalized amplitude $A^{*}=\sqrt{2} A_{R M S} / D$ and frequency $f^{*}=f / f_{N}$ of steady oscillations ( $A_{R M S}$ is the root mean square value of amplitude of oscillations and $f_{N}$ is the frequency of oscillations of the squaresection prism in still water). They found a quasi-linear dependence of $A^{*}$ with the reduced velocity $U^{*}=U /\left(f_{N} D\right)$ ( $U$ is the speed of the unperturbed flow), quite independent of the mechanical damping. They also found that a good convergence of data seems to occur when re-normalized quantities $A^{\prime}=A^{*} /\left(2 m^{*}\right)$ and $U^{\prime}=U^{*} /\left(4 \pi m^{*}\right)$ are introduced. More recently, Nemes et al. (2012) and Zhao et at. (2014) carried out additional experiments in a water channel for lower values of the mass ratio, $m^{*}=2.2$ and $m^{*}=2.6$ respectively. In these cases, normalized amplitude were reported as $A^{*}=A_{10} / D$, where $A_{10}$ is the mean of the top $10 \%$ of the peaks (Hover et al. 1998). They found a quasi-linear dependence of $A^{\prime}$ with $U^{\prime}$ except in a region where a 1:3 synchronization with vortex shedding fluid loading seems to occur. However, for a fixed value of $U^{\prime}$, values of $A^{\prime}$ measured by Nemes et al. (2012) and Zhao et at. (2014) seem to be higher than those observed by Bouclin (1977) (see Fig. 2.1). In other words, from Bouclin's data one finds that $A^{\prime} \approx 0.36 U^{\prime}$, but from Nemes's and Zhao's experiments (with a lower value of $m^{*}$ ) one finds that $A^{\prime} \approx 0.46 U^{\prime}$. This point was noted in Nemes et al. (2012) who suggested that the reason for the differences could be ascribed to the different experimental conditions, such as turbulence intensity, surface roughness, aspect ratio, end condition, and blockage ratio. This statement deserves its own discussion and that is the reason why a series of test is conducted in order to clarify whether, in this range of parameters, the constant of proportionality between $A^{\prime}$ and $U^{\prime}$ depends on $m^{*}$. In an attempt to get insight into this point an experimental campaign was carried out in a re-circulating free-surface water channel of the transverse-induced oscillations of a square-section prism for several values of the mass ratio, ranging from 2.2 to 14.4, and low mechanical damping. It has been found that $A^{\prime}$ not only depends on $U^{\prime}$ but also, to a lesser extent, on $m^{*}$. Since this two parameter dependency might be relevant for some practical design applications of marine and river structures, its functional behaviour has been characterized. Experimental results also allow to introduce a re-normalized plot where a reasonable convergence of all experimental data takes place, as well as to get insight on other questions, such as up to what extent the oscillations can be characterized in terms of time-averaged quantities (amplitude and frequency), at which reduced velocity oscillations are expected to start, or when the Quasi-steady hypothesis can be resorted to. 


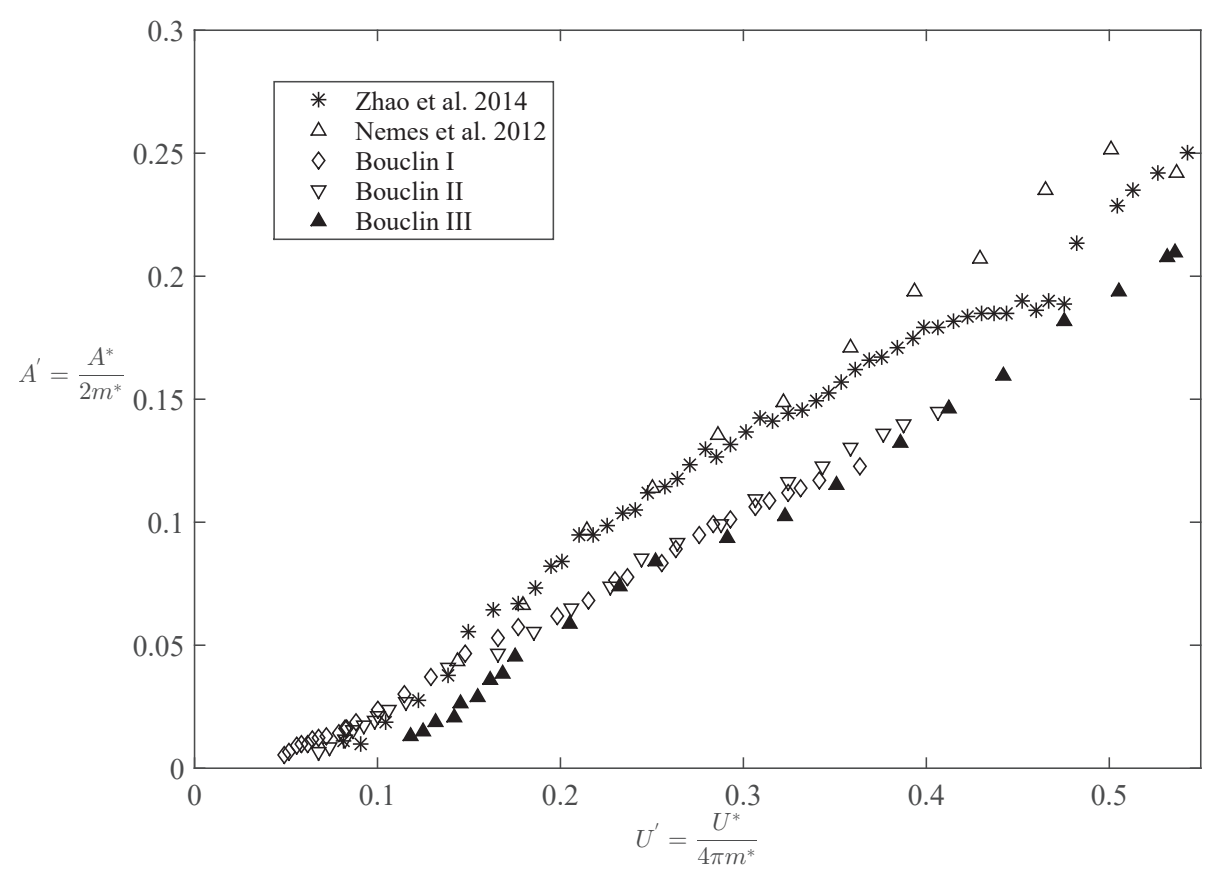

Figure 2.1: Variation of $A^{\prime}$ with $U^{\prime}$. Open diamonds represent $m^{*}=4.2$, filled triangles represent $m^{*}=8.5$ and inverted triangles represent $m^{*}=14$ from Bouclin (1977). $m^{*}$ was 2.2 in experiments from Nemes et al. (2012) and $m^{*}=2.6$ in Zhao et al. (2014).

The chapter is organized as follows: the experimental method and apparatus, acquisition procedures, and validation tests are detailed in the following section. Experimental results for the square section are presented and discussed in Section 2.3; an empirical law is proposed to estimate amplitude and frequency of oscillations as a function of both the reduced velocity and mass ratio. In addition, the level of fluctuations in the oscillations and the validity of the Quasi-steady hypothesis are discussed. Finally, concluding remarks are presented in Section 2.4 .

\subsection{Experimental set-up and validation}

\subsubsection{Experimental apparatus}

The experiments were carried out in a free-surface recirculating water channel with controlled inflow conditions at the test section in terms of mean speed, uniformity, and low turbulence. A sketch of the water channel is shown in Fig. 2.2. The water is driven by two equal axial pumps from ABS, model RCP 500 (maximum volume of flow $Q_{\max }=0.69 \mathrm{~m}^{3} / \mathrm{s}$, maximum pressure ratio $\triangle p_{\max }=13000 \mathrm{~Pa}$, maximum power consumption $\left.P_{\max }=11 \mathrm{~kW}\right)$. The rotation speed of the axial pumps is regulated by a variable frequency drive from Power Electronics (model SD503942) in such a way that the water channel velocity at the test section can be controlled in a range from $3 \mathrm{~cm} / \mathrm{s}$ to $110 \mathrm{~cm} / \mathrm{s}$. The test section is made of glass and allows for the flow to be 
viewed from either side, as well as the bottom. Guide vanes are placed in the corners in order to guide the flow and reduce pressure-losses. To improve the flow quality in the test section both a honeycomb (hexagonally shaped cells with a diameter of $4.5 \mathrm{~mm}$ and a length to diameter ratio of 12) and a screen are located before the entrance of the test section. The honeycomb is a very effective flow straightening device with a length above 10 cell diameters as shown by Bradshaw and Pankhurst (1964) and the screen is effective to reduce mean non-uniformities and fluctuations of the streamwise component.

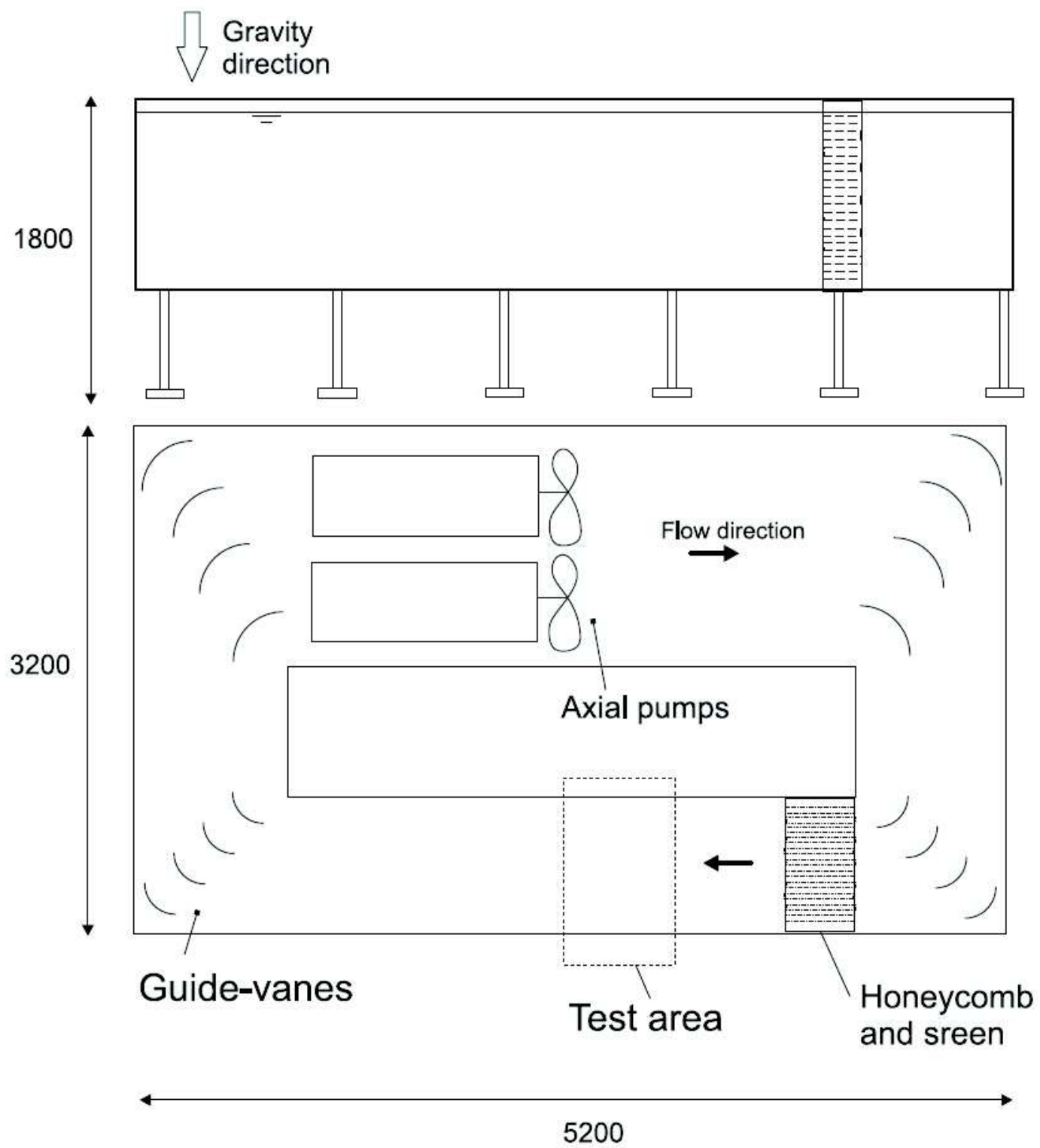

Figure 2.2: Schematic of the free-surface water channel. Dimensions are given in $\mathrm{mm}$.

A square-section prism (B) of side length $D=20 \mathrm{~mm}$, made of aluminium, was fixed vertically to the free end of a double-blade elastic system, which follows the arrangement introduced in Assi et al. (2006); see Fig. 2.3. The square-section prism had an immersed 
length $L$ of $820 \mathrm{~mm}$, and was furnished with a circular endplate (C) with diameter $75 \mathrm{~mm}$ to enhance two-dimensional flow. The elastic system was made up of with two parallel rigid aluminium blocks (D), coupled to a pair of thin spring-steel flexor blades (E). The elastic system not only acts as the prism support, but also provides the restoration response. In this way, the prism is restricted to oscillate in the transverse direction to the flow $(y)$ with low mechanical damping and high linear characteristics (additional details are provided in Appendix of the Chapter).

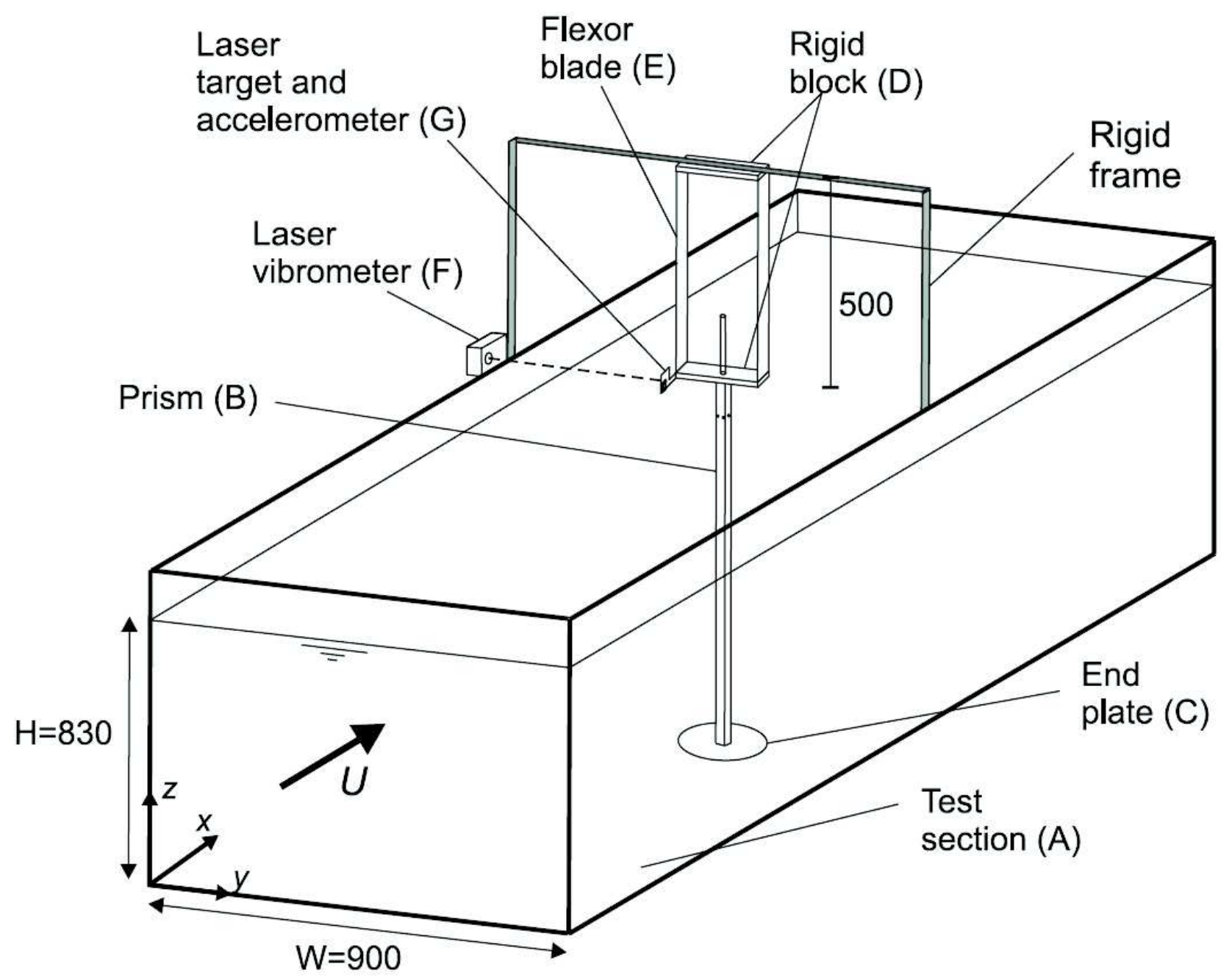

Figure 2.3: Sketch of the experimental set-up (all dimensions are in millimeters).

Fig. 2.4 shows is a detailed view of the galloping prism and the elastic system where the experiments were carried out. The flow speed was varied between $75 \mathrm{~mm} / \mathrm{s}$ and $400 \mathrm{~mm} / \mathrm{s}$, which gives a Reynolds number range of $R e=U D / \nu=1500-8000, \nu$ being the kinematic viscosity of the fluid. 


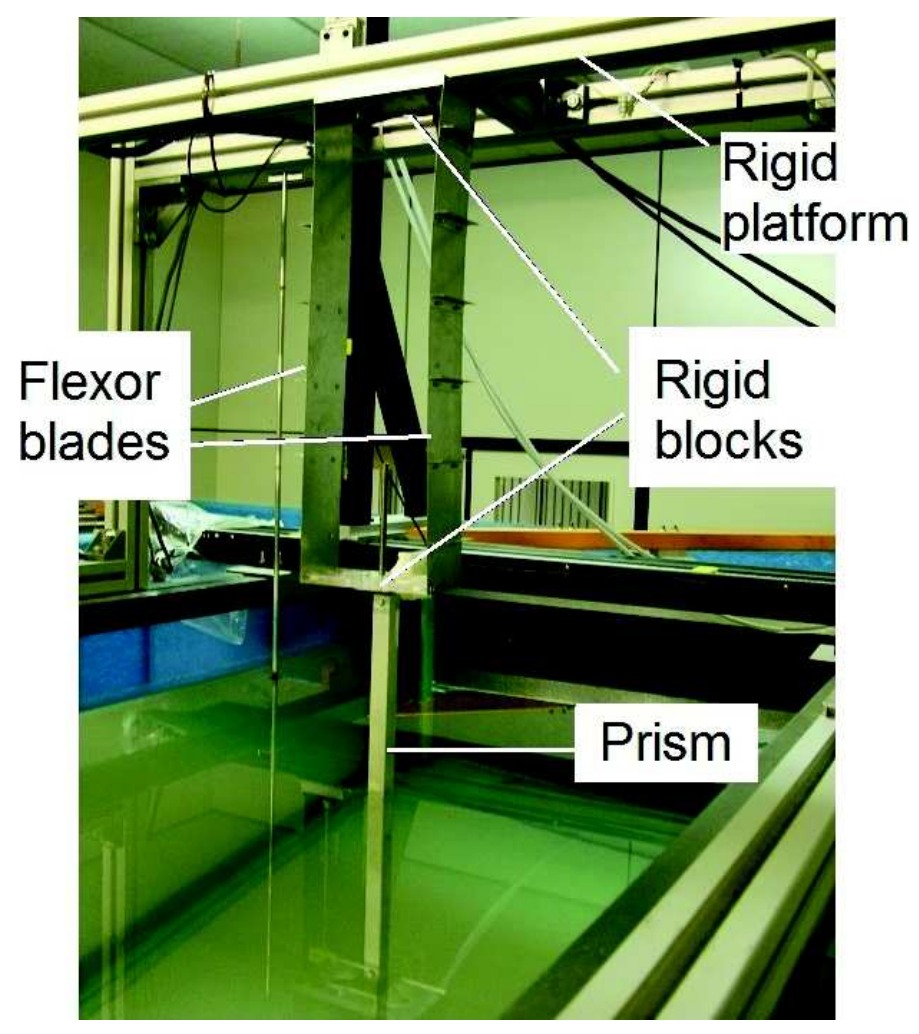

Figure 2.4: Photograph of a close view of the elastic system.

The flow velocity field across the working section was mapped for the empty channel (no prism mounted) employing a Pitot Tube and a differential pressure sensor from Druck (model LP1000). Local water velocity $u(y, z)$ was measured at $64(8 \times 8)$ points across the area of the test section for different values of the mean velocity $U$ within the range of study. Fig. 2.5a shows the dimensionless water velocity contours, defined as $\langle u(y, z)\rangle / U$ where $\langle\cdot\rangle$ stands for time-averaged, for a mean water speed of $0.36 \mathrm{~m} / \mathrm{s}$. Turbulence intensity, which is here defined as $T I=u^{\prime}(y, z) / U$ where $u^{\prime}$ is the root mean square of the velocity fluctuations, is shown in Fig. 2.5b. Water velocity profile is quite flat and vary less than $5 \%$ except in zones close to the walls. Turbulence level is below $2 \%$. This turbulence level is within the order of magnitude of other free-surface recirculating water channels employed by other research groups (Assi et al. 2009; Govardhan and Williamson, 2006; Radi et al. 2014). In addition, the turbulence spectrum does not show any predominant frequency over the frequency range of interest for the experiments. Zero inflow turbulence conditions may be achieved using a towing tank but, in this case, there is a limited time for each run due to the finite length of the tank (Hover et al. 1997).

Transverse displacement and acceleration of the square-section prism was measured by a non-contact laser vibrometer (F) from Aquity AR500-500 (range $500 \mathrm{~mm}$, resolution 0.05 $\mathrm{mm}$ ) and by an accelerometer $(\mathrm{G})$ from Measuring Specialities (range $\pm 2 \mathrm{~g}$, resolution $2 \mathrm{mg}$ ), and registered using a Compact-Rio microprocessor from National Instruments. The sampling frequency was $200 \mathrm{~Hz}$. During experiments, the maximum measured peak-to-peak amplitude of oscillation was $60 \mathrm{~mm}$, being much larger (by a factor of 1200) than the vibrometer resolution. 

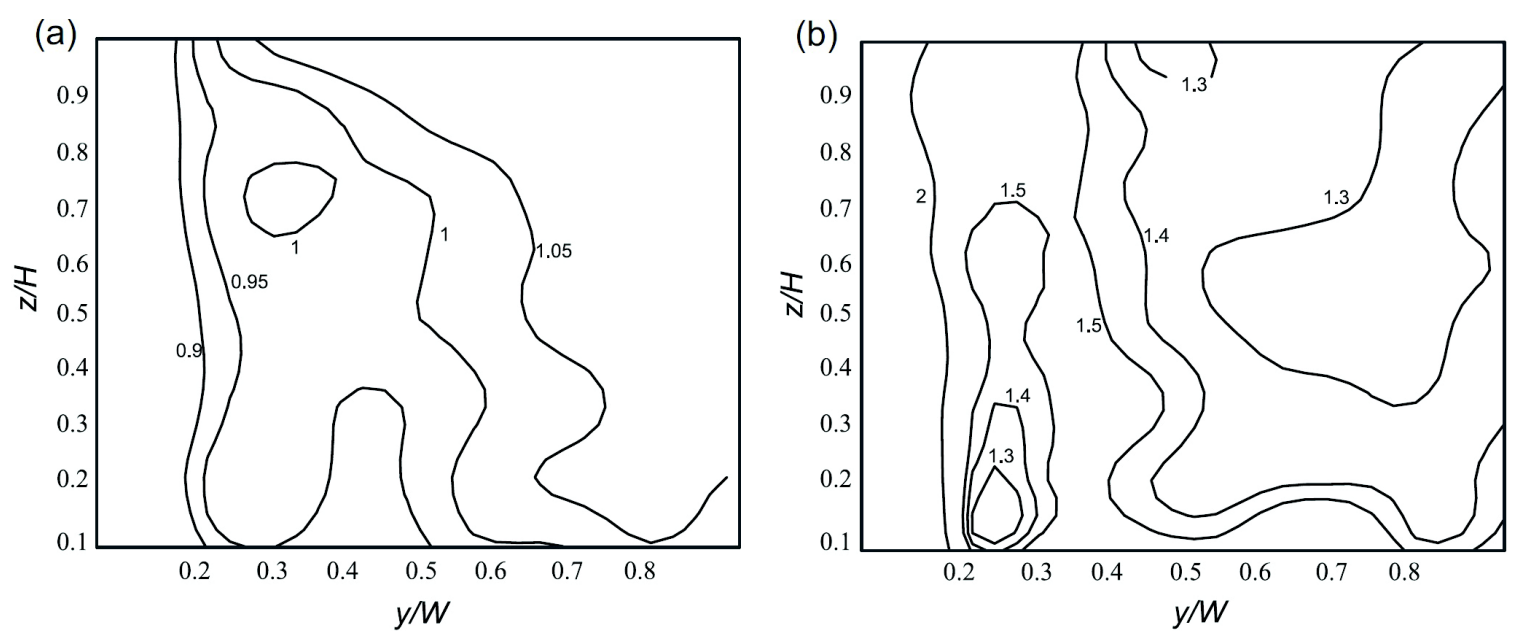

Figure 2.5: Dimensionless velocity distribution (a) and turbulence intensity distribution (\%) (b) at the test section for a mean water velocity $\mathrm{U}$ of $0.36 \mathrm{~m} / \mathrm{s}$.

For each parameter combination of flow speed velocity $\left(U^{*}\right)$ and mass $\left(m^{*}\right.$; note that mass ratio can be increased adding masses on the lower block of the elastic system, see Appendix at the end of the Chapter), data were recorded for a total of $10 \mathrm{~min}$. The first $8 \mathrm{~min}$ were spent on achieving steady state conditions while the remaining two minutes were used to recording. After that, the water speed was increased and the measurement procedure repeated. Regarding repeatability of the results, a series of cases characterized by a well-defined steady state harmonic behaviour were selected and different time series of measurements were taken. It was found that the oscillation amplitude differences were smaller than $3 \%$.

\subsubsection{Data reduction}

For each run, the instantaneous position $y(t)$ and acceleration $\ddot{y}(t)$ of the prism was directly measured and registered. The instantaneous fluid force $F(t)$ was computed from the equation dynamics of the prism, as was proposed in Zhao et al. (2014) and Govardhan and Williamson (2000):

$$
m \ddot{y}+c \dot{y}+k y=F(t)=-m_{A} \ddot{y}+\frac{1}{2} \rho U^{2} D L C_{Y},
$$

where $m$ is the total oscillating mass, $c$ the mechanical damping constant, and $k$ the stiffness of the system. Both $c$ and $k$ were measured in free decay tests in still air (empty water channel). More details of these tests are presented in Annex. Regarding the right hand side of Eq. 2.1, note that the fluid force $F(t)$ has been split into two terms, an added mass term $\left(-m_{A} \ddot{y}\right)$ and a viscous one $\left(\rho U^{2} D L C_{Y} / 2\right)$, as suggested in Govardhan and Williamson (2000); $\rho$ is the fluid density, and $C_{Y}$ a dimensionless fluid force coefficient.

The prism oscillation velocity $\dot{y}$ was computed by first order numerical integration of $\ddot{y}$ and fifth order band-pass Butterworth filtering; the cut-off frequencies of the Butterworth filter were 
$1 / 10$ and 10 times the frequency of oscillation in such a way that the introduced lag is negligible. Added mass $m_{A}$ was obtained from free-decay tests in still water since $m_{A}=m\left[\left(f_{A} / f_{N}\right)^{2}-1\right]$ where $f_{A}$ is the frequency of oscillations in still air and $f_{N}$ in still water (Zhao et al. 2014). Observe that $F(t)$ can be computed from the left-hand side of Eq. 2.1, after all mechanical properties are known and the response is measured. Note that $C_{Y}(t)$ can also be computed from Eq. 2.1.

The equation of the dynamics of the prism can be made dimensionless introducing $D$ and $f_{N}^{-1}$ as representative length and time scales. This gives

$$
\ddot{Y}+2 \zeta \dot{Y}+Y=\frac{U^{* 2}}{8 \pi^{2}\left(m^{*}+m_{A}^{*}\right)} C_{Y},
$$

where $Y=y / D, \zeta=c /\left(2\left(k\left(m+m_{A}\right)\right)^{1 / 2}\right)$ is the damping ratio, and $m^{*}=m /\left(\rho D^{2} L\right)$ is the mass ratio. The dots above $Y$ are the derivative with respect to dimensionless time $\tau=2 \pi f_{N} t$ now.

An usual method of analysis employed for the study of FIV problems is based on the introduction of time-averaged quantities. In this sense the following variables were computed from each run:

- The steady normalized maximum amplitude of oscillation $A^{*}=A_{10} / D$.

- The normalized frequency of oscillations $f^{*}=f / f_{N}$ where the frequency of oscillations $f$ was computed from Fast Fourier Transform of $y(t)$.

- Fluid force coefficient $C_{Y}$ and lift force coefficient $C_{L}=2 F /\left(\rho U^{2} D L\right)$.

- Phase delay $\phi$ between the prism displacement $y(t)$ and the fluid force $F(t)$. This phase delay has been computed by means of Hilbert Transform computation of $F(t)$ and $y(t)$.

- Fluid force coefficient in phase with the prism velocity $C_{V}=\overline{C_{Y} \dot{Y}} / \overline{\dot{Y}^{2}}$, where overline stands for averaged quantity, and fluid force coefficient in phase with the prism acceleration $C_{A}=\overline{C_{Y} \ddot{Y}} / \overline{\ddot{Y}^{2}}$

- A correlation coefficient to quantify to what extent the amplitude of oscillations has a steady character, given by $H_{A}=\sqrt{2} A_{R M S} / A_{10}$. Values of $H_{A}$ close to unity denote a well defined steady state with nearly fixed amplitude of oscillations, whereas lower values indicate fluctuations of the amplitude over the time.

\subsubsection{Validation test}

To validate the experimental set-up and to obtain reference data for comparison, a preliminary experiment was performed with a circular cylinder with diameter $25 \mathrm{~mm}$ and submerged length of $820 \mathrm{~mm}$. Mass ratio $\left[\mathrm{m}^{*}=4 \mathrm{~m} /\left(\rho D^{2} L \pi\right)\right]$ was 2.4 , damping $\zeta=0.003$, the aspect ratio was 32.8 and blockage ratio $2.7 \%$. Figure 2.6 shows the measured VIV response in terms of $A^{*}$ 
$\left(A_{10}^{*}\right)$. For comparison, results of Assi et al. (2006) are also shown. Assi's experiments were carried out in the water channel from Imperial College (width $700 \mathrm{~mm}$, depth $600 \mathrm{~mm}$ ), and similar conditions than experiments in this Thesis in terms of mass ratio $m^{*}=1.9$, low damping $(\zeta=0.007)$, blockage ratio (4.5), aspect ratio (26), and Reynolds number range (3000-12,000).

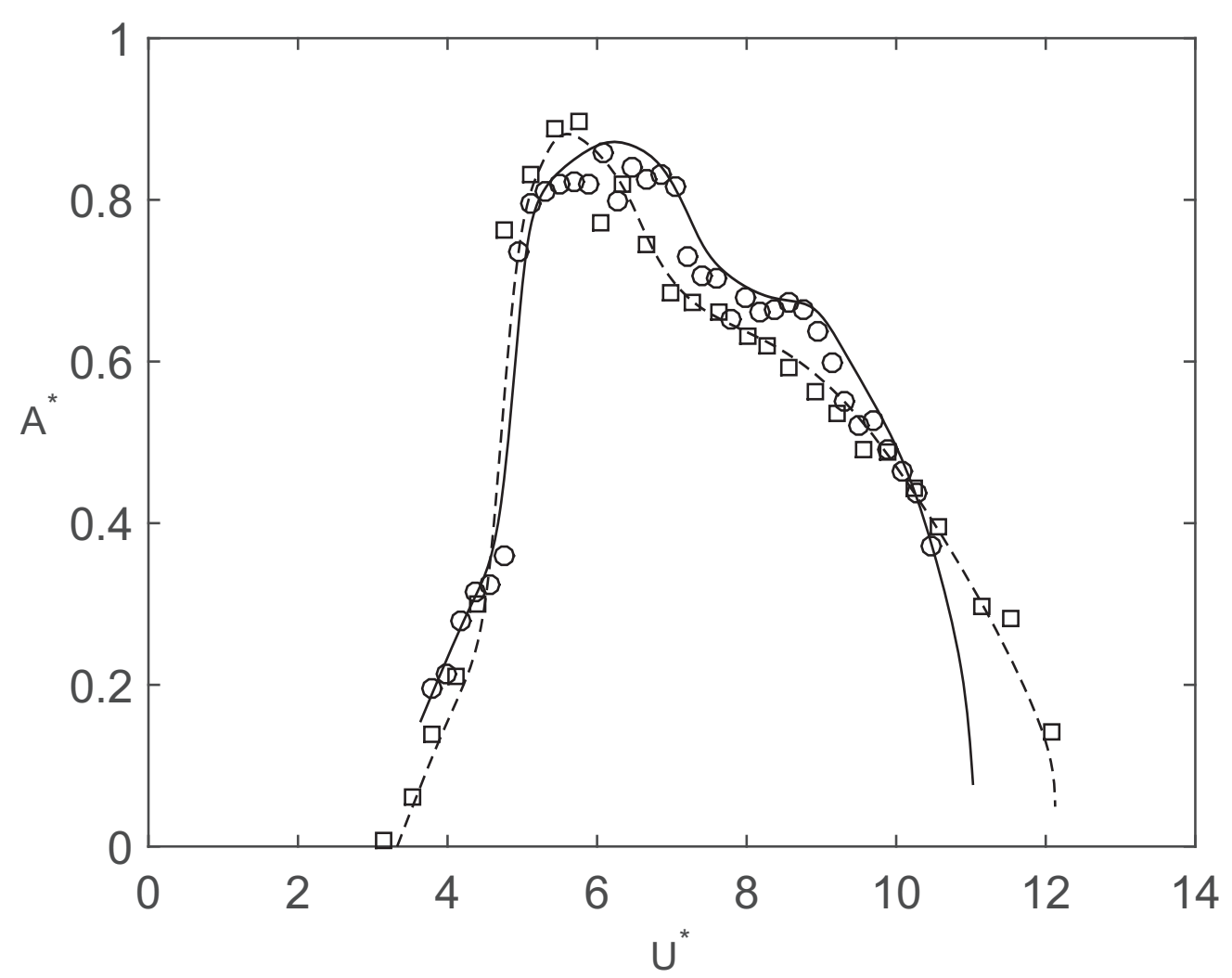

Figure 2.6: Normalized amplitude variation with reduced velocity in VIV of a circular cylinder. Open circles stand for present results $\left(m^{*}=2.4\right)$ and open squares are from Assi et al. (2006) $\left(m^{*}=1.9\right)$.

Good overall agreement between experimentally obtained results and Assi's data can be noted, and a number of common characteristics can be distinguished. First, the initial branch around $U^{*}=5$, where amplitude grows very steeply. Then, amplitude grows with the reduced velocity in the upper branch until a maximum value is achieved at $U^{*} \approx 6$, drops slowly in a range of reduced velocities $U^{*}=6-10$ (the lower branch), and finally drops steeply in the region of de-synchronization for $U^{*}=10-12$.

Finally, Figure 2.7 shows the variation of the amplitude of oscillations and the RMS total lift force coefficient $C_{L, R M S}=2 F_{R M S} /\left(\rho U^{2} D L\right)$ with the reduced velocity. In addition, values given in Hover et al. (2004) are also shown for comparison. Hover's experiments were carried out in a towing tank with a circular cylinder of mass ratio of $m^{*}=3$, damping ratio was $\zeta=0.032$ blockage ratio $4.5 \%$, and aspect ratio 26 . 
(a)

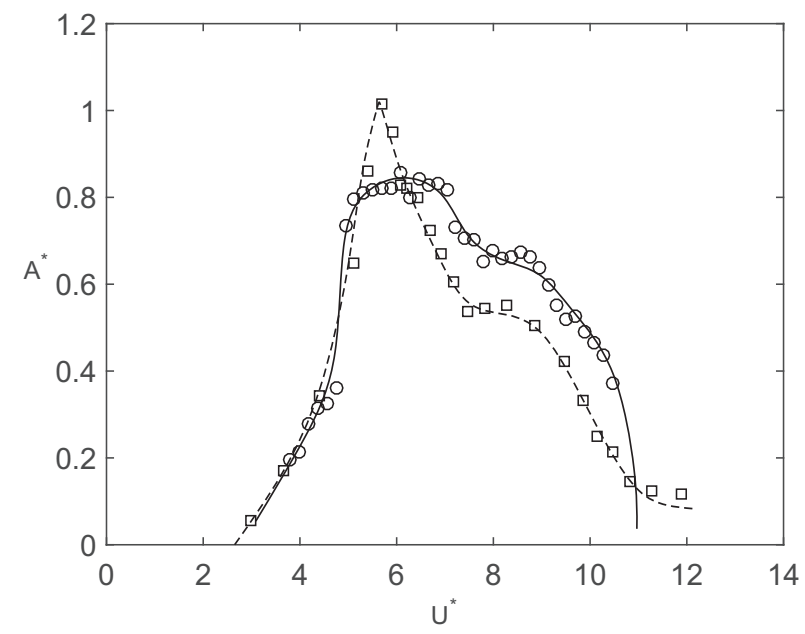

(b)

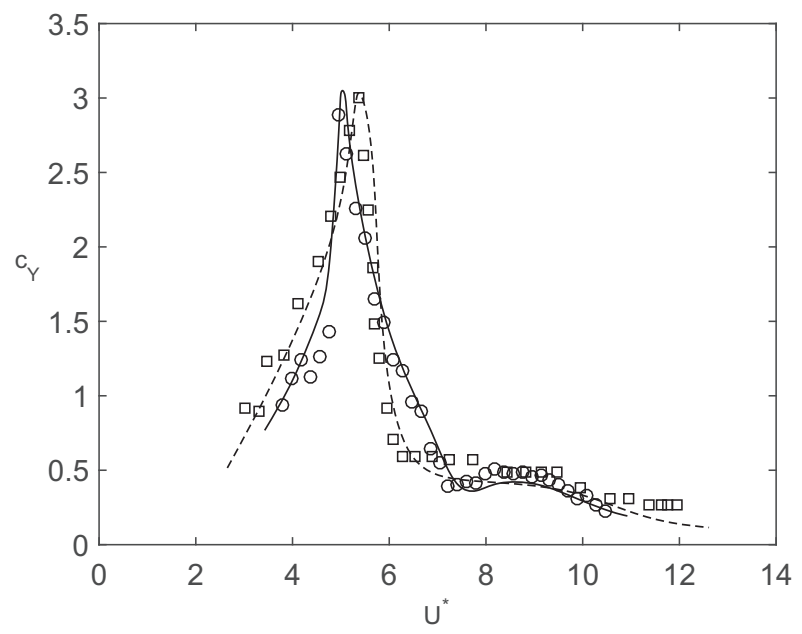

Figure 2.7: (a) Normalized amplitude variation with reduced velocity in VIV of a circular cylinder. Open circles represent present results $\left(m^{*}=2.4\right)$ and open squares from Hover et al. (2004) $\left(m^{*}=3, \zeta=0.032\right)$. (b) RMS total lift force coefficient variation with the reduced velocity from the same experiments.

\subsection{Results}

For the square-section prism five values of $m^{*}$ were tested. Table 2.1 shows physical values for each configuration as well as the value of the different dimensionless parameters.

Table 2.1: Mechanical data in the five configurations studied for the square-section prism.

\begin{tabular}{|c|c|c|c|c|c|}
\hline Configuration & 1 & 2 & 3 & 4 & 5 \\
\hline Total oscillating mass $m(\mathrm{~kg})$ & 0.72 & 1.72 & 2.72 & 3.72 & 4.72 \\
\hline Natural frequency in water $f_{N}(\mathrm{~Hz})$ & 0.99 & 0.91 & 0.87 & 0.85 & 0.84 \\
\hline Natural frequency in air $f_{N a}(\mathrm{~Hz})$ & 1.34 & 1.06 & 0.97 & 0.92 & 0.89 \\
\hline Mass ratio $m^{*}$ & 2.2 & 5.2 & 8.3 & 11.3 & 14.4 \\
\hline Damping ratio $\zeta$ & 0.0030 & 0.0023 & 0.0019 & 0.0017 & 0.0015 \\
\hline Mass-damping parameter $\left(m^{*}+m_{A}^{*}\right) \zeta$ & 0.012 & 0.016 & 0.019 & 0.022 & 0.022 \\
\hline
\end{tabular}

\subsubsection{Amplitude response}

As already said, five different values of $m^{*}$ have been tested maintaining fixed other influencing parameters like end-conditions, blockage ratio, aspect ratio of the prism, turbulence characteristics of the inflow, or the Reynolds number range. Figure 2.8a shows $A^{*}\left(A_{10}^{*}\right)$ dependence with the reduced velocity $U^{*}$. In all cases the growth of the amplitude of oscillations with the 
reduced velocity is monotonic. It can be noted that as $m^{*}$ is lower, oscillations start at a lower reduced velocity. It is also noted that the linear trend is interrupted over a certain value of reduced velocity, and a "kink" effect appears. The value of $U^{*}$ at which the "kink" effect appears decreases with $m^{*}$ whereas the range of reduced velocity where "kink" effect is observed increases with the mass ratio. This effect has been associated to a $1: 3$ synchronization effect between the frequency of oscillations and the frequency of vortex shedding (Bearman at al. 1987, Nemes et al. 2012, Zhao et al. 2014). Note how this "kink" effect is observed more clearly for low $m^{*}$.

Figure $2.8 \mathrm{~b}$ shows $H_{A}$ dependence with reduced velocity. The "harmonicity" (understood here as the situation at which the oscillation is purely sinusoidal with fixed amplitude) increases with the reduced velocity until a saturation value is achieved, and seems to be quite independent of the mass ratio. However, for the lowest mass ratio, $H_{A}$ departs from the general behaviour at reduced velocities where the "kink" effect takes place suggesting a connection between the loss of "harmonicity" and vortex shedding. 
(a)

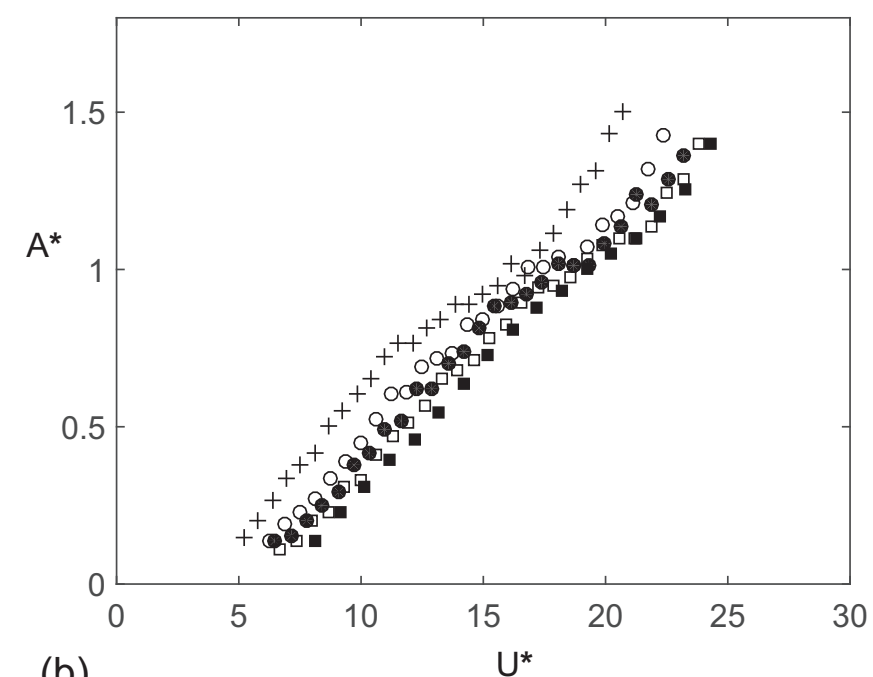

(b)

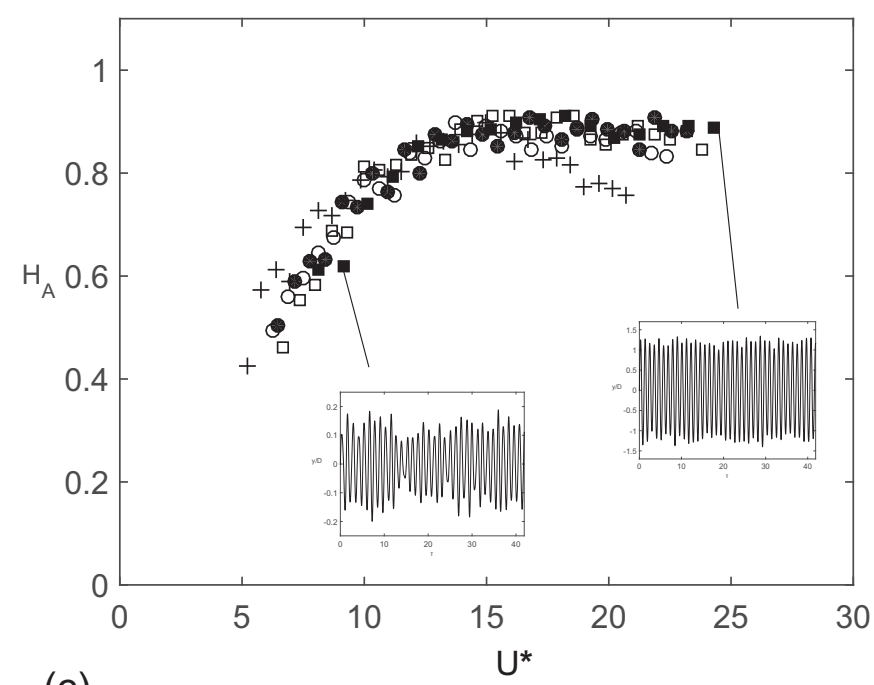

(c)

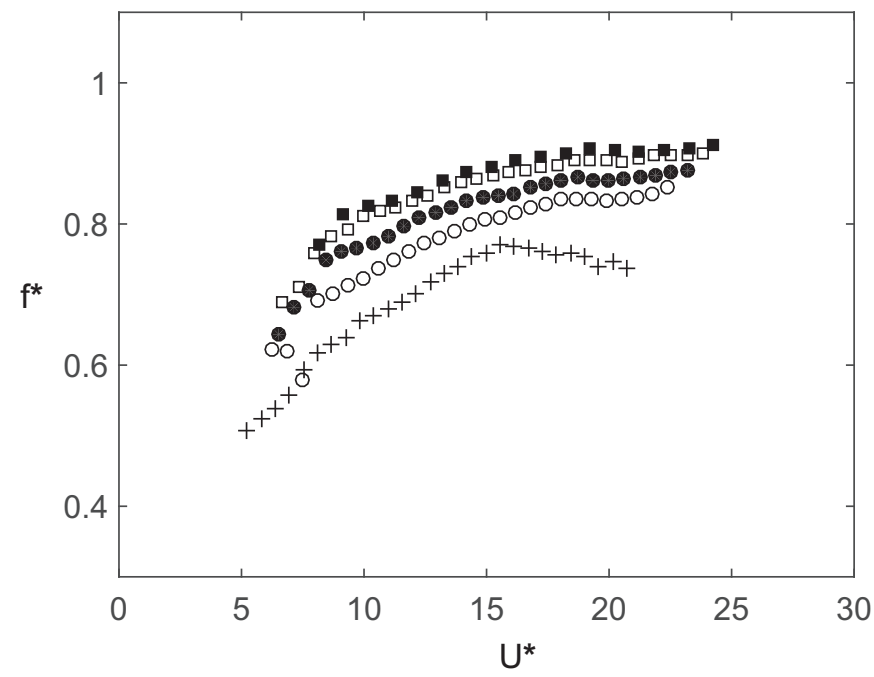

Figure 2.8: Variation of the normalized amplitude $A^{*}$ (a), $H_{A}$ correlation coefficient (b), and normalized frequency of oscillations $f^{*}(\mathrm{c})$, with reduced velocity. Filled squares denotes $m^{*}=$ 14.4, open squares $m^{*}=11.3$, filled circles $m^{*}=8.3$, open circles $m^{*}=5.2$, and crosses $m^{*}=2.2$. 


\subsubsection{Frequency response}

Frequency response is shown in Fig. 2.8c. The behavior is qualitatively similar for all values of $m^{*}$. As can be seen, the frequency of oscillations is lower for low values of the reduced velocity and grows with $U^{*}$, more slowly as $U^{*}$ increases. For a fixed reduced velocity, the dimensionless frequency of oscillations is lower as $m^{*}$ diminishes.

In order to understand the observed frequency behaviour the fluid force coefficient acting on the square-section prism along the transverse direction $C_{Y}$ is split in terms of components in phase with acceleration and velocity of the oscillation; thus, from Eq. 2.2

$$
\ddot{Y}+2 \zeta \dot{Y}+Y=\frac{U^{* 2}}{8 \pi^{2}\left(m^{*}+m_{A}^{*}\right)}\left(C_{A} \ddot{Y}+C_{V} \dot{Y}\right),
$$

and the normalized frequency of oscillations will be therefore,

$$
f^{*}=\left(\frac{1}{1-\frac{U^{* 2} C_{A}}{8 \pi^{2}\left(m^{*}+m_{A}^{*}\right)}}\right)^{1 / 2}
$$

which will be known when information about $C_{A}$ is available. Figure 2.9a shows a plot of the fluid force coefficient $C_{A}$ computed from experimental results (remember that $C_{A}=\overline{C_{Y} \ddot{Y}} / \ddot{\ddot{Y}^{2}}$ ). It seems that $C_{A}$, on the range of values of $U^{*}$ and $\left(m^{*}+m_{A}^{*}\right)$ covered by this experimental campaign, depends more strongly on the reduced velocity than on $\left(m^{*}+m_{A}^{*}\right)$. The relations between $C_{A}$ and $U^{*}$ and $\left(m^{*}+m_{A}^{*}\right)$ are found to be approximated by a monomial function

$$
C_{A}=K U^{* \alpha}\left(m^{*}+m_{A}^{*}\right)^{\beta}
$$

where $K=-2783, \alpha=-3$ and $\beta=0.3$ provide the best least square fit. Substituting this empirical result into Eq. 2.4 one arrives that

$$
f^{*}=\left(\frac{1}{1+\frac{35}{U^{*}\left(m^{*}+m_{A}^{*}\right)^{0.7}}}\right)^{1 / 2}
$$

which is shown graphically in Figure 2.9b. This result indicates that (i) for high mass ratio $f^{*}$ tends to one, which is a well known result; (ii) for a fixed reduced velocity the frequency of oscillations is smaller for lower values of the mass ratio; (iii) $f^{*}$ grows with the reduced velocity and tends asymptotically to one. 
(a)

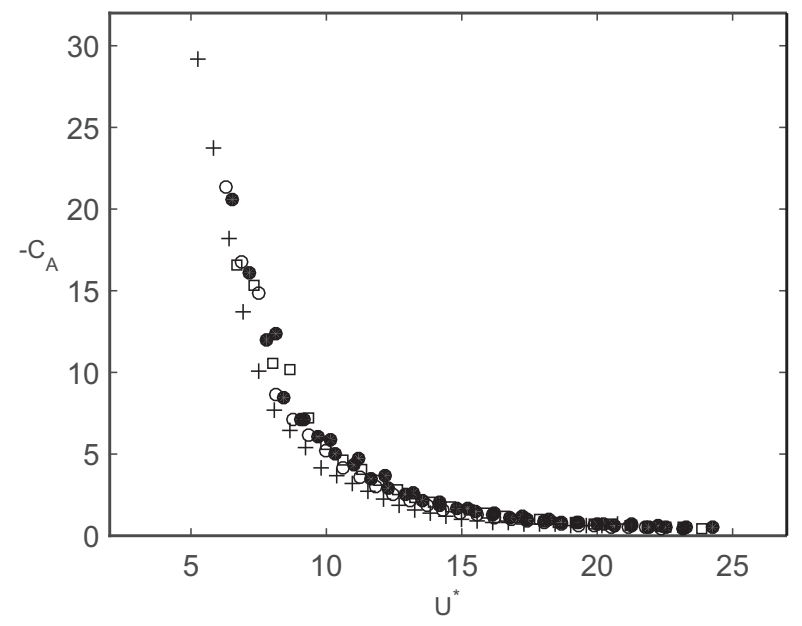

(b)

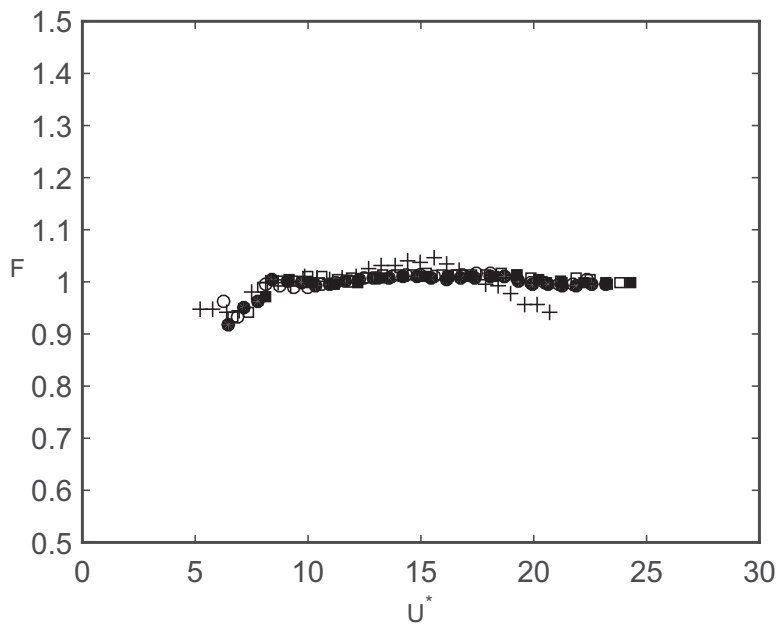

Figure 2.9: (a) Dependence of fluid force coefficient in phase with prism acceleration with reduced velocity for all tests. (b) $F=f^{*} / f_{C O M P}^{*}$ variation with reduced velocity from tests; $f^{*}$ is the measured reduced frequency, $f_{C O M P}^{*}$ is given by Eq. 2.6. Filled squares denotes $m^{*}=14.4$, open squares $m^{*}=11.3$, filled circles $m^{*}=8.3$, open circles $m^{*}=5.2$, and crosses $m^{*}=2.2$.

\subsubsection{Re-normalized amplitude response}

The dissimilar response plots of amplitude shown in Figure 2.8a can be re-normalized onto a single response curve by a suitable normalization of the velocity. Figure 2.10 shows $A^{*}$ dependence with the "true" reduced velocity $V^{*}=U /(f D)=U^{*} / f^{*}$ from obtained experiments. Now a unique curve is available for amplitude response for each value of $V^{*}$. To my knowledge, this might be the first time that this specific convergence of data is reported. 


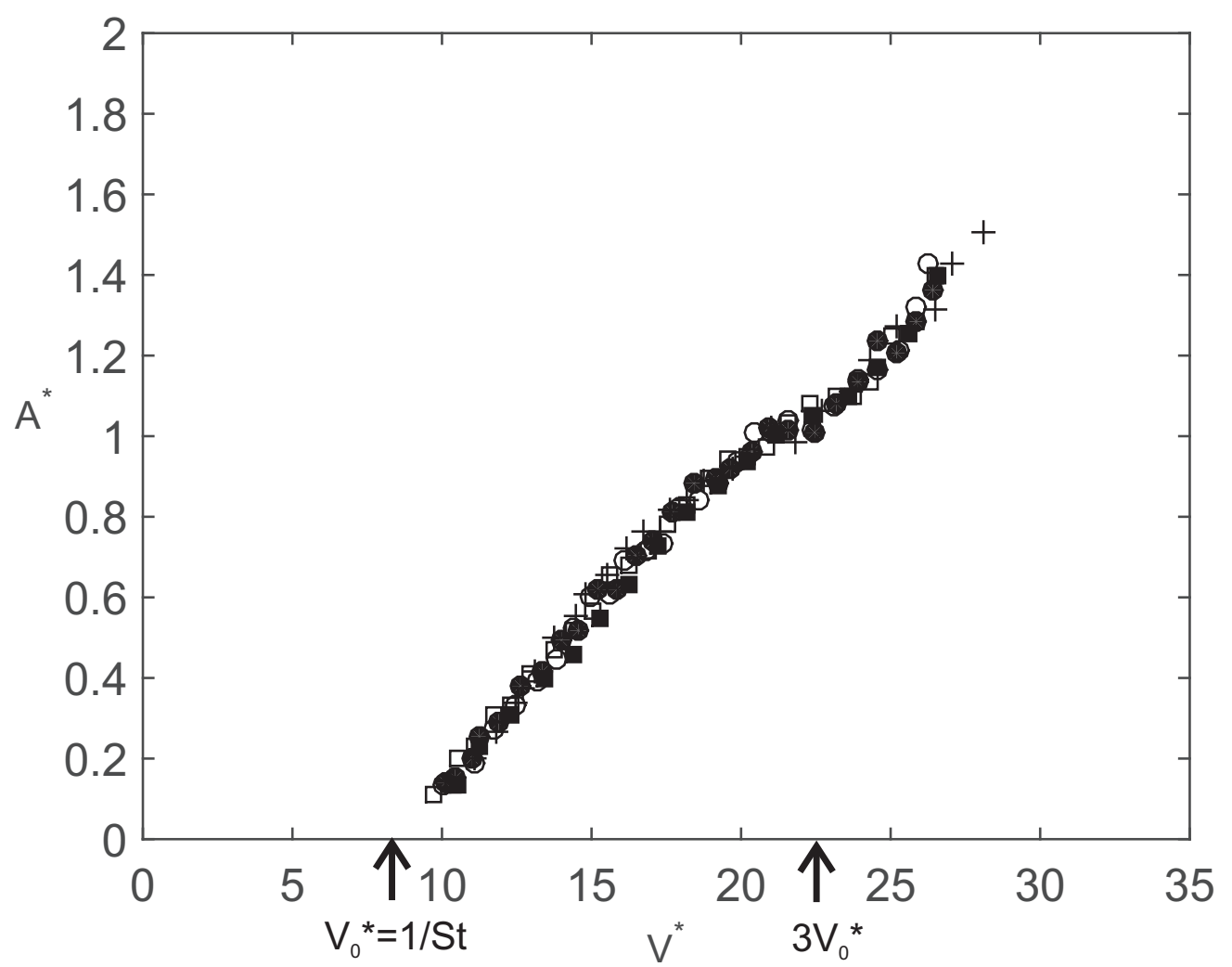

Figure 2.10: Normalized amplitude variation with true reduced velocity. Filled squares denotes $m^{*}=14.4$, open squares $m^{*}=11.3$, filled circles $m^{*}=8.3$, open circles $m^{*}=5.2$, and crosses $m^{*}=2.2$.

A tentative explanation of this result can be made here: when the flow speed is different from zero the characteristic time scale of the problem used to make the fluid velocity dimensionless $\left(f_{N}^{-1}\right)$ has no physical meaning, since the "true" time in the problem is the time of actual oscillations $f^{-1}$. In this aspect it makes sense that $V^{*}=U /(f D)=U^{*} / f^{*}$ is a the appropriate parameter.

From Fig. 2.10 it seems that oscillations cannot start below a certain value $V_{0}^{*} \approx 7.7 \approx \mathrm{St}^{-1}$ where St is the Strouhal number of a fixed square section (around 0.13, Paidoussis et al. 2011). From Fig. 2.8a one may see that the classical reduced velocity at which oscillation starts $U_{0}^{*}$ depends on $m^{*}$, but also it may be seen there is a dependence with $m^{*}$ for the frequency of oscillations at this stating point $f_{0}^{*}$ (see Fig. 2.8c), in such a way that $V_{0}^{*}=$ $U_{0}^{*} / f_{0}^{*}$ tends to a fixed value, which is the reciprocal of the Strouhal number. This result was anticipated by Bearman and Luo (1988) in their investigation where fluid forces were measured from forced vibration experiments. In addition a marked linear relationship between $A^{*}$ and $V^{*}$ can be noted, slightly modified at $V^{*} \approx 3 V_{0}^{*}$. Nemes et al. (2012) have suggested that a 1:3 synchronization between the frequency of oscillations and the frequency of vortex shedding synchronization) may be responsible for this modification of the linear trend.

Now, approaching the amplitude response by a linear law, 


$$
A^{*} \approx 0.6\left(\frac{V^{*}}{V_{0}^{*}}-1\right)
$$

Therefore, $A^{*} / V^{*} \approx 0.6\left(1 / V_{0}^{*}-1 / V^{*}\right)$ tends asymptotically to a fixed value of $0.6 / V_{0}^{*}$ for growing values of true reduced velocity. As it was pointed out by Bearman and Luo (1988), $A^{*} / V^{*}$ is physically linked to the maximum instantaneous angle of attack between the body and the flow, since $\beta_{M A X}=\tan ^{-1} \dot{y}_{M A X} / U \sim \tan ^{-1}\left(2 \pi A^{*} / V^{*}\right)$. Then it follows that $\beta_{M A X}$ approaches to a fixed value for large values of the true reduced velocity $V^{*}$.

For completeness, Figure 2.11 shows $A^{*}$ dependence with the "true" reduced velocity $V^{*}=$ $U /(f D)=U^{*} / f^{*}$ from previous experimental results along with present results. A reasonable agreement can be observed. However, differences are expected to appear because of the different experimental conditions (end conditions, blockage ratio, aspect ratio, turbulence characteristics of the inflow, etc.).

(a)

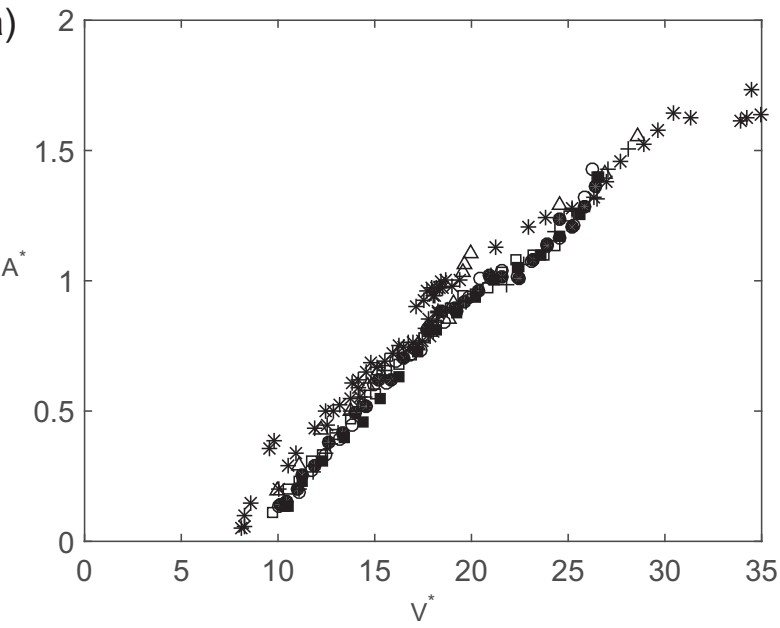

(b)

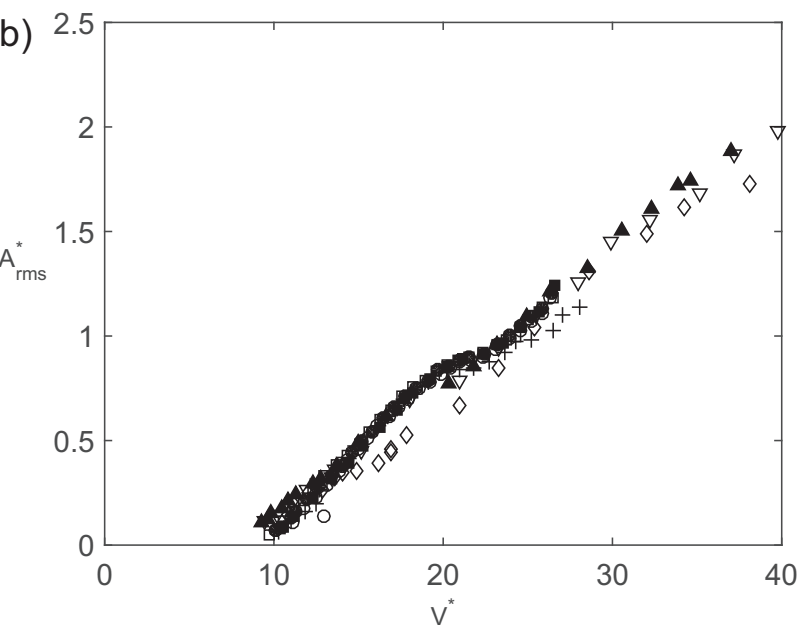

Figure 2.11: (a) Normalized amplitude response of several experiments $\left(A^{*}=A_{10}^{*}\right)$ as a function of true reduced velocity; open triangles stand for Nemes et al. (2012) results, stars for Zhao et al. (2014). (b) Normalized amplitude variation $\left(A^{*}=\sqrt{2} A_{R M S}^{*}\right)$ with true reduced velocity; Bouclin and Parkinson results: open diamonds $\left(m^{*}=4.2\right)$, filled triangles $\left(m^{*}=8.5\right)$, and open inverted triangles $\left(m^{*}=14\right)$. In both figures, present results are presented by filled squares $\left(m^{*}=14.4\right)$, open squares $\left(m^{*}=11.3\right)$, filled circles $\left(m^{*}=8.3\right)$, open circles $\left(m^{*}=5.2\right)$, and crosses $\left(m^{*}=2.2\right)$.

\subsubsection{Amplitude response in primitive variables}

From practical standpoint, $V^{*}$ is not a satisfactory parameter since it is only defined once the solution is known. In this sense, it would be preferable to rewrite Eq. 2.7 in terms of independent variables which are known a priori. To this end, from Eq. 2.7 and taking into account $V^{*}=U^{*} / f^{*}$ 


$$
A^{*} \approx 0.6\left(\frac{U^{*}}{f^{*} V_{0}^{*}}-1\right)
$$

which can be developed further taking into account Eq. 2.6

$$
A^{*} \approx 0.6\left(\frac{U^{*}}{V_{0}^{*}}\left(\frac{1}{1+\frac{35}{U^{*}\left(m^{*}+m_{A}^{*}\right)^{0.7}}}\right)^{-1 / 2}-1\right)
$$

This expression can be useful to predict approximately the transverse flow-induced oscillations of a square prism in hydroelastic situations (low mass ratio) with low damping. It shows that, for a fixed value of the reduced velocity $U^{*}, A^{*}$ is expected to be higher for lower values of $m^{*}$ as it is apparent from the comparison of experiments by Nemes et al. (2012) and Zhao et al. (2014) with those of Bouclin and Parkinson (1977) and the present results. It also shows that $A^{*}$ is almost linearly proportional to the reduced velocity for high enough values of $U^{*}\left(m^{*}+m_{A}^{*}\right)$ whereas for low values the amplitude is not linearly related to the reduced velocity and scales with $U^{* n}$ where $n$ lays between $1 / 2$ and $1\left(1 / 2\right.$ in the limit of $\left.U^{*}\left(m^{*}+m_{A}^{*}\right) \ll 1\right)$.

Moreover, amplitude slope can be obtained from Eq. 2.9, by differentiating $A^{*}$ with respect to $U^{*}$ and taking into account that $V_{0}^{*}=7.7$, one obtains

$$
\frac{\partial A^{*}}{\partial U^{*}}=\frac{0.078 Z+1.36}{\left(Z^{2}+35 Z\right)^{1 / 2}}
$$

with $Z=U^{*}\left(m^{*}+m_{A}^{*}\right)^{0.7}$. It can be seen that for large values of $Z$ (that is, either $U^{*}$ or $\left(m^{*}+m_{A}^{*}\right)$ are large or both of them are) the amplitude is expected to be linearly related to the reduced velocities $\left(\partial A^{*} / \partial U^{*} \approx 0.078\right)$.

Coming back to Eq. 2.9, the reduced velocity at which oscillations start, $U_{0}^{*}$, can be obtained from Eq. 2.9 setting $A^{*}=0$ and solving. Figure 2.12 shows that the observed values of $U_{0}^{*}$ in the present experiments (see Fig. 2.8a) and previous experimental results agree approximately with the value computed numerically from Eq. 2.9 setting $A^{*}=0$. As it was anticipated from experiments, when the mass ratio $m^{*}$ is diminished oscillations starts at a lower flow velocity. 


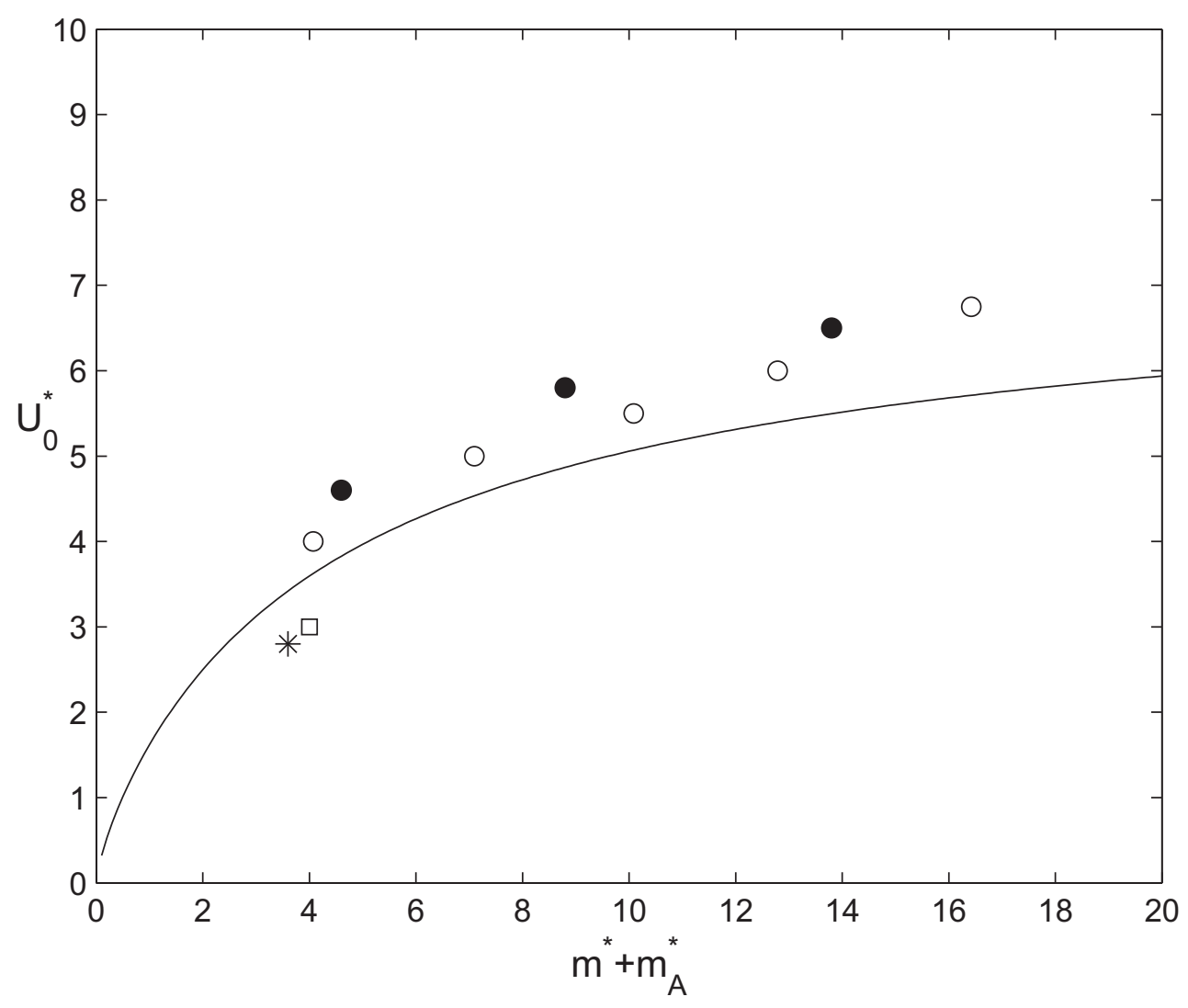

Figure 2.12: Variation with $m^{*}+m_{A}^{*}$ of the reduced velocity at which oscillations starts $U_{0}^{*}$. Solid line represents the value of $U_{0}^{*}$ computed from Eq. 2.9 setting $A^{*}=0$; open circles stand for present results, filled circles results from Bouclin (1977), star and open square stand for, respectively, results from Nemes et al. (2012) and Zhao et al. (2014).

\subsubsection{Phase delay: Quasi-steady conditions}

After analysing the phase delay $\phi$ measured during experiments, it is worth to note that, in all cases, the phase lag $\phi$ is close to zero degrees, away from Quasi-steady conditions $\left(\phi=90^{\circ}\right)$, even for large values of $U^{*}$ (and $V^{*}$ ). This reinforces the idea that the reduced velocity may not be the only parameter to be accounted for when the assessing of the validity of the Quasi-steady hypothesis, as it is often assumed. This question was already pointed out by Bearman and Luo (1988) and Bearman et al. (1987).

Considering this point here, the dynamics of the square prism is

$$
m \ddot{y}+c \dot{y}+k y=F(t) .
$$

If steady-state of sinusoidal oscillations with amplitude $A$ and frequency $f$ are assumed, $y(t)=A \sin (2 \pi f t)$, and the fluid force is modelled as $F(t)=F_{0} \sin (2 \pi f t+\phi)$ with $F_{0}$ constant and $\phi$ the phase delay between the displacement and the fluid force $F(t)$. Equating sine and 
cosine terms in Eq. 2.11 it is possible to find that

$$
\phi=\tan ^{-1}\left(\frac{2 \zeta f^{*}}{1-\left(\frac{m^{*}}{m^{*}+m_{A}^{*}}\right) f^{* 2}}\right) .
$$

Substituting the empirical law found from experiments for the frequency of oscillations $f^{*}$, Eq. 2.6, it comes

$$
\phi=\tan ^{-1}\left(\frac{2 \zeta Z^{1 / 2}(Z+35)^{1 / 2}}{Z(1-M)+35}\right)=\tan ^{-1}(G),
$$

where $Z=\left(U^{*}\left(m^{*}+m_{A}^{*}\right)^{0.7}\right), M=m^{*} /\left(m^{*}+m_{A}^{*}\right)$ and for the sake of simplicity the whole expression inside the parenthesis is called $G$. This expression suggests that the phase angle depends not only on the reduced velocity $U^{*}$ but also on the mass ratio $m^{*}$ and the mechanical damping $\zeta$ (note that all these parameters are grouped into the variable $G$ ). Figure 2.13 shows $\phi$ variation with $G$. As can be shown, phase delay approaches to $\phi=90^{\circ}$ (Quasi-steady conditions) as $G$ increases. Almost Quasi-steady conditions are met for $G \geq 10$. Figure 2.13 also shows $\phi$ delay measurements from wind tunnel experiments of Bearman et al. (1987), which is the only reference in the literature available where phase delay is measured in free vibration experiments. Even though there is a poor quantitative agreement (note also the considerable scatter in experimental data), it can be noted that $\phi$ measurements from Bearman et al. (1987) follow the trend given by Eq. 2.13, supporting the idea that governing parameters $U^{*}, m^{*}$ and $\zeta$ should all be taken into account to determine if the Quasi-steady hypothesis is applicable. $G$ increases with $U^{*}, m^{*}$ and $\zeta$ so that for low or moderate values of $m^{*}$ (say, hydroelastic situations) Quasi-steady conditions should not formally appear unless either the reduced velocity or the mechanical damping (or both) be high enough. On the contrary, for aeroelastic situations where $m^{*}$ is usually very large it is possible to achieve Quasi-steady conditions. For, example for $\left(m^{*}+m_{A}^{*}\right)=1000, \zeta=0.01$ and $U^{*}=100, G$ takes a value of 7.7 and, according to Eq. 2.13, $\phi=83^{\circ}$.

\subsection{Conclusions}

Transverse flow-induced oscillations of a square prism at low mass ratio and low damping have been experimentally studied. This campaign has enlarged existing experimental results published in the literature and, in the range of the parametric space covered, has shown the following:

1. It appears to be confirmed that the amplitude of oscillations seem to be linearly dependent on the reduced velocity, where the constant of proportionality between reduced velocity and amplitude of oscillations depends on the mass ratio.

2. Oscillations start at a reduced velocity which depends on the mass ratio; when the mass ratio is diminished oscillations start at a lower reduced velocity. However, in terms of 


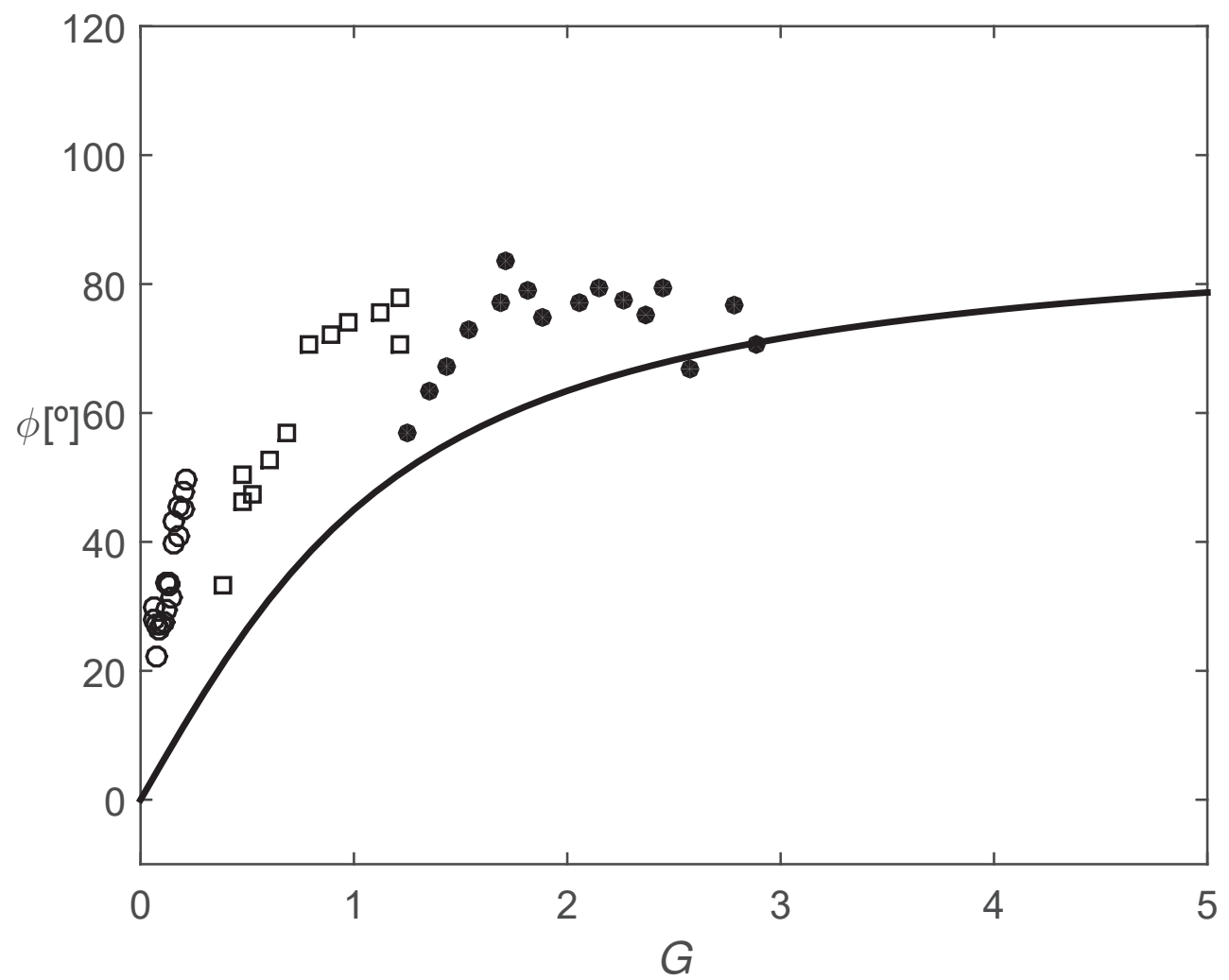

Figure 2.13: Phase delay prediction from Eq. 2.13 (solid line). Phase delay measurements in wind tunnel experiments $\left(m^{*}=1013\right)$ by Bearman et al. (1987) (see Figure 8 of that paper) are also shown: open circles $(\zeta=0.0009)$, open squares $(\zeta=0.003)$, and filled circles $(\zeta=0.006)$.

true reduced velocity, oscillations seems to start at a fixed threshold value, which is very close to $\mathrm{St}^{-1}$ where $\mathrm{St}$ is the Strouhal number of vortex shedding for the square prism at rest.

3. A convergence of experimental data seems to have taken place when the amplitude of oscillations is plotted against the true reduced velocity.

4. The phase lag between oscillations and fluid force is close to zero even for large values of the reduced velocity. This suggests that the question of whether Quasi-steady conditions work seems to be non-trivial and cannot be answered solely looking at the value of the reduced velocity. Taking into account the experimental law found for the frequency of oscillations it was found that the phase angle depends not only on the reduced velocity $U^{*}$ but also on the mass ratio $m^{*}$ and the mechanical damping $\zeta$. Quasi-steady conditions are approached as all these three parameters increase. To further study this point, the development of controlled high damping ratio experiments might be of interest.

5. Concerning the question of steadiness of oscillations it has been observed that the amplitude of oscillations vary with time when vortex shedding effects are strong, in the range of true reduced velocity of $V^{*}=7.7-13$. In this regime, any description of the dynamics of 
the square prism (say, amplitude and frequency of oscillations) and flow quantities (say, fluid force, phase lag) by time-averaged variables does not seem to be appropriate.

6. From the engineering side, in offshore metallic (low damping) structural members with square cross-section, the expression given in Eq. 2.9 can constitute a guide to estimate the level of stiffness required in the structure to avoid transverse oscillations.

\section{Appendix}

\section{Annex I: Elastic system}

As introduced in Section 2.2, there is some advantages when the elastic system based on flexor blades is employed. With a single element it is possible to get (i) restriction of the prism motion mainly in the transverse direction (note that a small vertical motion is allowed, $z \sim y^{2} / L_{B}$, as can be seen in Fig. 2.14a, (ii) restoring force, (iii) low inherent damping, and (iv) linearity.

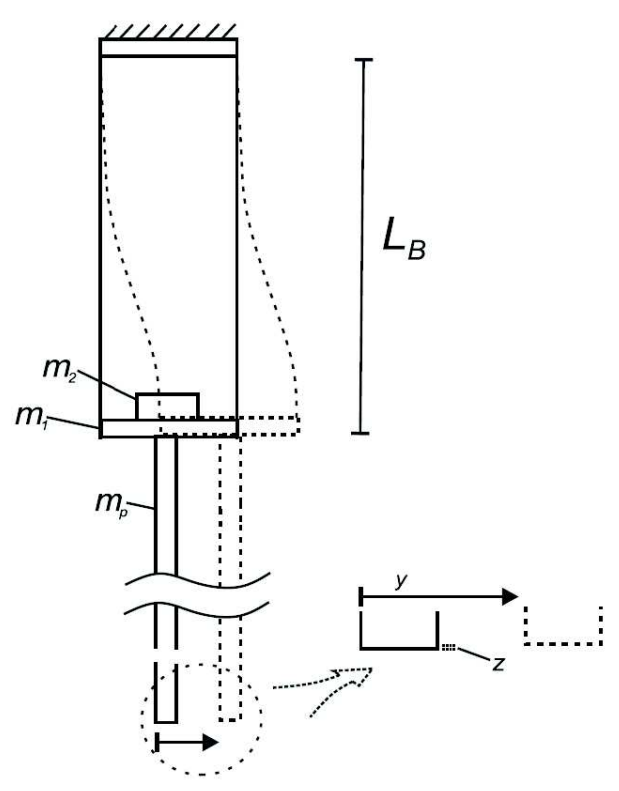

(a)

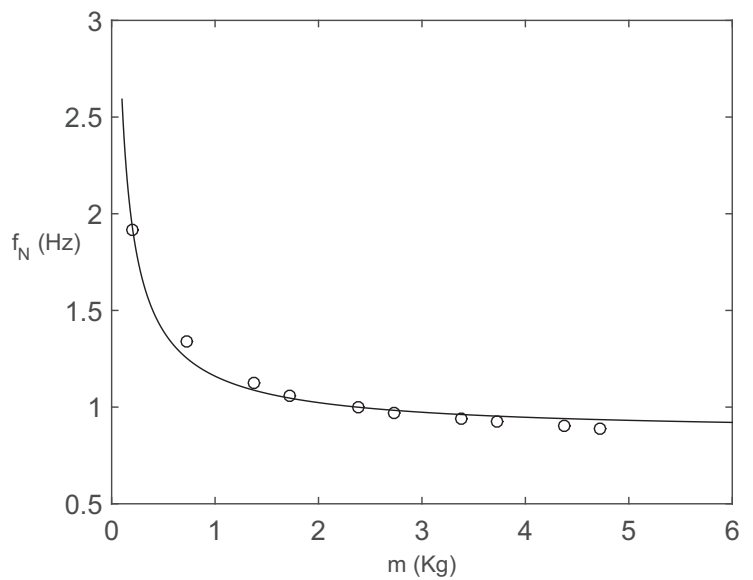

(b)

Figure 2.14: (a) Detail of the elastic system. (b) Natural frequency of oscillations variation with total mass: open circles are experimental results natural, solid line stand for the elastic pendulum equation.

Restoring force has two distinct contributions, the first one comes from the rigidity of the blades whereas the second one comes from the effect of gravity on the tip mass acting like a pendulum. Therefore, the natural frequency of oscillations in vacuum of the elastic system should be given by: 


$$
f_{0}=\frac{1}{2 \pi} \sqrt{\frac{k_{B}}{m_{T}}+g L_{B}}
$$

where $k_{B}$ is the total stiffness constant of the blades, $m=m_{1}+m_{2}+m_{p}$ is the total mass of the system, $g$ is the gravity constant, and $L_{B}$ the length of the blade (see Fig. 2.14a).

In order to characterize the behaviour of the elastic system, free decay tests in still air (empty water channel) were carried out. Fig. 2.14b shows the natural frequency of oscillations measured experimentally with different values of total mass (it is changed by adding tip mass $m_{2}$ ). As it can be seen experimental results matches well with mathematical expression given above. It was also checked the linearity of the system. As an example, Fig. 2.15a shows results from a free decay where $m=3.72 \mathrm{Kg}$. After applying the Hilbert transform to the free decay signal it can be seen that frequency of oscillations and damping ratio remain quite constant along the amplitude of oscillations, which shows the high degree of linearity of the system. Frequency of oscillation has a variability less than $0.5 \%$. In the case of damping ratio the differences are under $\Delta \zeta= \pm 0.0001$.
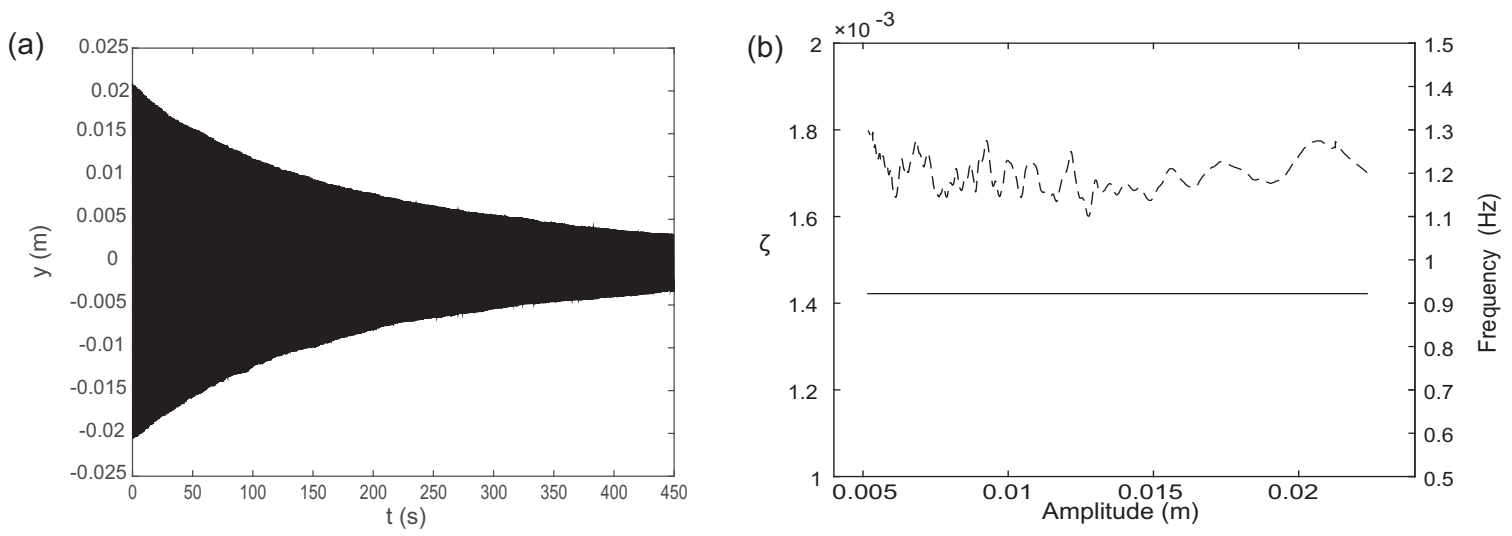

Figure 2.15: (a) Free decay oscillations for $m=3.72 \mathrm{Kg}$. (b) Frequency of oscillations (solid line) and damping ratio (dashed line) variation with the amplitude of oscillation. 


\section{Chapter 3}

\section{Quasi-steady time delayed model}

\subsection{Introduction}

As anticipated in Chapter 2, predictions of Flow-induced Vibrations for a square-section prism given by the Quasi-steady model are satisfactory compared with experimental results in aeroelastic situations, where the mass ratio $m^{*}$, defjined as the mean density of the prism divided by the density of the fluid, is very large, and mechanical damping is moderate. However, in hydroelastic situations, when $m^{*}$ is of order unity, experiments (Nemes et al. 2012; Zhao et al. 2014), and new experiments presented in Chapter 2 and published in Xu-Xu et al. (2016b), suggest that the Quasi-steady model fails to reproduce some features:

- The frequency of oscillations $\omega$ may departs significantly from $\omega_{N}$.

- The phase lag between the fluid force and oscillations is not $90^{\circ}$ (even for large reduced velocities).

- There is a linear relationship between the amplitude of oscillations and the reduced velocity (as the Quasi-steady model predicts) but the constant of proportionality seems to depend on the mass ratio being larger as the mass ratio diminishes.

- For very low values of mass-damping parameter (close to zero) oscillations start at a certain value of reduced velocity, higher than zero, which is not the value predicted by the Quasi-steady modelling.

- Oscillations can occur below the reduced velocity at which the resonance condition for Vortex Induced Vibrations takes place. In other words, oscillations may start at reduced velocities significantly lower than $S t^{-1}$ where $S t$ is the stationary Strouhal number).

In addition, Luo and Bearman $(1988,1990)$ did a detailed experimental campaign carried out by means of both forced and free vibration experiments (with very low damping) over a square prism. After that, they suggested that quasi-steady conditions were not related with the reduced velocity $U^{*}$ but rather related with the parameter $U^{*} \omega^{*} / A^{*}$ (where $\omega^{*}=\omega / \omega_{N}$ is the normalized frequency and $A^{*}=A / D$ is the normalized amplitude of oscillations). They also stated that quasi-steady conditions take place only for large values of $U^{*} \omega^{*} / A^{*}$.

In this Chapter, a Quasi-steady time-delayed model that is able to reproduce the most notable features of TG in the whole range of the mass ratio $m^{*}$ is presented. The idea of introducing a time delay in the Quasi-steady modelling is not new, and it was considered in 
the analysis of the fluid-elastic instability of a cylinder array (Price and Paidoussis, 1984; or Hemon, 1999). In this case a time delay is introduced, expressed as

$$
\tau=\mu D / U
$$

where $\mu$ is a free parameter to be chosen ( $D$ is the characteristic dimension of the body and $U$ the unperturbed inflow velocity). The time delay $\tau$ is due to the time taken by the flow around the body to respond to changes in the body motion in such a way that the fluid force is described by its quasi-steady value evaluated at time $t-\tau$. The key point (and the weakness) of this model is how to fix $\tau$ since usually $\tau$ is selected in the literature after the response (solution) of the system is known.

Following the idea of time delay, a theoretical model has been developed for the analysis of the Flow-induced Oscillations of a square-section prism. The main novelty of this work is the proposal of an analytical expression that links $\tau$ to static aerodynamic characteristics that fixes $\tau$ in advance, from known parameters. In spite of the model, as will be shown, it is only a crude approximation that is able to reproduce, at least qualitatively, experimental observations, which should always be kept in mind as the main objective of the model. It is hard to think that rather a simple theoretical model would be able to capture all the physics of the problem. The model emerges as an intent to explain experimental results presented in Chapter 2 and is intending by none means to predict Vortex-Induced Vibrations of circular cylinders. In this last case, there appears to be some strong lock-in phenomena between the frequency of vortex shedding and the cylinder motion in such a way that the cylinder and wake responses result very complex.

In Section 3.2 the mathematical model is developed, and comparisons with experimental results from both free and forced vibration experiments are presented in Section 3.3. In spite of the fact that the comparisons with existing experimental results (including those of the Chapter 2) are not bad from a quantitative point of view, it should be outlined that the model helps to explain qualitatively all the phenomenology above mentioned that are not explained by the classic Quasi-steady theory. For example, the model gives a solid argumentation about when quasi-steady conditions are expected to be hold. Finally, concluding remarks are given in Section 3.4.

\subsection{Quasi-steady time delayed model}

A one-degree-of-freedom model is introduced to describe the transverse displacement of the spring-mounted galloping body. It is based on the equilibrium between inertia, damping, and stiffness forces, as well as the transverse fluid force. That is,

$$
m \ddot{y}+c \dot{y}+k y=\frac{1}{2} \rho U^{2} D C_{Y}(t),
$$


where $y$ denotes the transverse position of the prism, $m$ is the mass per unit length of the body, $c$ is a mechanical damping constant per unit length, $k$ is the stiffness constant per unit length, $\rho$ is the fluid density, $U$ is the undisturbed velocity of the incident flow, $D$ the side length of the body's cross-section and $L$ its length, and $C_{Y}$ is the instantaneous fluid force coefficient in the transverse direction to the incident flow. Finally, the dot symbol stands for differentiation with respect to physical time $t$.

\subsubsection{Classical Quasi-steady modelling}

As said earlier, in the mathematical model of TG it is common to describe $C_{Y}$ by means of the Quasi-steady hypothesis, since TG is typically a low-frequency oscillation phenomenon where the characteristic timescale of the prism oscillation is much larger than the characteristic timescale of the flow. Then, the flow around the body is assumed to adapt instantaneously, without any time delay, to the motion of the body and the fluid force is only dependent on the instantaneous attitude of the prism with respect to the incident flow, which can be described by the instantaneous angle of attack $\alpha: C_{Y}(t)=C_{Y}(\alpha(t))$. For the oncoming theoretical analysis, to maintain a compromise between the complexity and the accuracy of the development of the theory, a cubic polynomial is considered enough (see Blevins 1990, p. 130) to approximate the vertical aerodynamic force coefficient dependence with $\tan \alpha$, so

$$
C_{Y}(\alpha)=a_{1} \tan \alpha+a_{3} \tan ^{3} \alpha
$$

where $a_{1}$ (>0 in galloping bodies) and $a_{3}(<0)$ are empirical coefficients got after fitting $C_{Y}$ versus $\tan (\alpha)$ by a polynomial. $C_{Y}$ versus $\tan (\alpha)$ dependence was measured in static tests (experimental or numerical). The values of $a_{1}$ and $a_{3}$ depend on the cross-section geometry of the prism. In addition, from Fig. 3.1 it follows that

$$
\tan (\alpha)=\frac{\dot{y}}{U}
$$

and therefore, the fluid force can be expressed as a polynomial of non-dimensional velocity of oscillations

$$
C_{Y Q S}(t)=a_{1}\left(\frac{\dot{y}}{U}\right)+a_{3}\left(\frac{\dot{y}}{U}\right)^{3},
$$

where $C_{Y Q S}$ is the Quasi-steady value of the transverse fluid force coefficient. As can be seen $C_{Y Q S}$ is in-phase with the velocity of oscillations $(\dot{y})$, so that the phase lag between the fluid force and the body's oscillation is $90^{\circ}$. Eqs. 3.1 and 3.4 give the dynamics of the body under Galloping if the Quasi-steady hypothesis is resorted to. Considering characteristic length $D$ and time $t^{*}=\omega_{N}^{-1}$, where $\omega_{N}$ is the circular frequency of oscillations in still fluid, the dimensionless equation for the dynamics of the body is found as

$$
Y^{\prime \prime}+2 \zeta Y^{\prime}+Y=\frac{U^{* 2}}{2 m^{*}}\left(a_{1} \frac{Y^{\prime}}{U^{*}}+a_{3} \frac{Y^{\prime 3}}{U^{* 3}}\right)
$$


where $\zeta=c /\left(2 m \omega_{N}\right)$ is the damping ratio, $U^{*}=U /\left(\omega_{N} D\right)$ is the reduced velocity, $m^{*}=$ $m /\left(\rho D^{2}\right)$ is the mass ratio, $Y=y / D$ and the comma stands differentiation with respect to the dimensionless time. Under harmonic oscillations the normalized amplitude is $A_{Q S}^{*}=A_{Q S} / D$ and the normalized frequency of oscillations is $\omega_{Q S}^{*}=\omega_{Q S} / \omega_{N}$. From Eq. 3.5 in combination with the approximation $\sin ^{3}\left(\omega^{*} t^{*}\right) \approx 3 \sin \left(\omega^{*} t^{*}\right) / 4$ and equating sine and cosine terms, it follows that

$$
A_{Q S}^{*}=\left(\frac{4 U *}{3 a_{3}}\left(4 m^{*} \zeta-a_{1} U^{*}\right)\right)^{1 / 2} \quad \omega_{Q S}^{*}=1
$$

Observe that oscillations take place when $U^{*}>U_{0}^{*}=4 m^{*} \zeta / a_{1}$.

\subsubsection{Time delayed Quasi-steady modelling}

A time delay term $\tau$ is introduced due to the amount of time taken for the flow around the body to respond to changes in the body motion in such a way that the fluid force coefficient is described by Quasi-steady value evaluated at time $t-\tau$. The physical reasoning behind this argumentation is that the flow around the body at time $t$ has not enough time to adapt the instantaneous angle of attack $\alpha(t)$ and is assumed to be the same to that angle of attack of the delayed time $t-\tau$. Then,

$$
C_{Y}(t)=C_{Y Q s}(t-\tau) \approx C_{Y Q S}(t)-\tau \frac{d C_{Y Q S}(t)}{d t}+\frac{\tau^{2}}{2} \frac{d^{2} C_{Y Q S}(t)}{d t^{2}},
$$

for small values of $\tau$. From Eq. 3.4

$$
C_{Y}(t)=a_{1}\left(\frac{\dot{y}}{U}\right)+a_{3}\left(\frac{\dot{y}}{U}\right)^{3}-\frac{a_{1} \tau \ddot{y}}{U}+\frac{a_{1} \tau^{2} \dddot{y}}{2 U},
$$

where only linear terms has been retained in $\tau$ and $\tau^{2}$ for simplicity (terms in $\dot{y} y, \dot{y}^{2} \ddot{y}$, and $\dot{y}^{2} \ddot{y}$ are neglected). That is, only the first damping correction term and the first stiffness correction term, both due to unsteady effects, are considered.

Now, the question is to how to model $\tau$. It is reasonable to assume that $\tau$ depends on the ratio of the convective time and the oscillation time. When convective time is small compared to the oscillation time, the time delay should approach to zero. The point is that two different characteristic timescales can be identified in the problem associated to two different characteristic velocities in the problem, the unperturbed flow speed $U$ and the velocity of oscillations $\dot{y}$. Therefore, two different reduced velocities can be identified, one is defined with the flow speed $V^{*}=U /(\omega D)$ where $\omega$ is the circular frequency of oscillations and the other defined with the velocity of oscillations $W^{*}=\dot{y} /(\omega D)$. Under harmonic oscillations of amplitude $A$ and frequency $\omega$, peak value of $W^{*}$ is $A^{*}$. It is reasonable to think that the time delay will have a dependence with both reduced velocities $V^{*}$ and $W^{*}$. Then, a monomial relationship is assumed for the non-dimensional time delay, 


$$
\tau^{*}=\gamma V^{* N} A^{* M}
$$

where $\tau^{*}=\tau \omega_{N}, \gamma$ is a constant, and $N$ and $M$ need to take negative values since time delay should approaches to zero as the reduced velocity gets higher (convective time small compared to oscillation time).

From experimental results in hydroelastic situations, it is observed that $A^{*} \approx \sqrt{-4 a_{1} /\left(3 a_{3}\right)} V^{*}$. Taking this into account, from Eq. 3.9,

$$
\tau^{*}=\gamma C^{M} V^{* N+M}
$$

with $C=\sqrt{-4 a_{1} /\left(3 a_{3}\right)}$ which is positive.

Taking, as a first trial $N=M=-1$, taking into account that $V^{*}=U^{*} / \omega^{*}$ where $\omega^{*}=\omega / \omega_{N}$ and taking, rather crudely, that $\gamma=1$ then

$$
\tau^{*}=\frac{\omega^{* 2}}{C U^{* 2}}
$$

Now, introducing an additional consideration which consists in approximate $\omega^{* 2}$ as

$$
\omega^{* 2} \approx\left(1+C_{M} / m^{*}\right)^{-1},
$$

where $C_{M}$ is the added mass coefficient under potential flow. Then it follows that

$$
\tau^{*}=\frac{1}{K U^{* 2}}
$$

with

$$
K=\sqrt{\frac{-4 a_{1}}{3 a_{3}}}\left(1+C_{M} / m^{*}\right)
$$

The advantage of the proposed time delay is that there is no empirical coefficient to adjust but information of the cross-section geometry is introduced via $K\left(a_{1}, a_{3}\right.$, and $\left.C_{M}\right)$ and the mass ratio. On the other hand, some drawbacks are expected. For example, $\tau^{*}$ grows without limit as $U^{*}$ diminishes which does not have physical sense. For reduced velocities close to stationary Strouhal number, the interaction between vortex shedding and body motion is expected to occur by introducing a complex picture that probably would not be captured with a single time delayed model.

Introducing Eq. 3.14 into Eq. 3.8 and taking $D$ and time $t^{*}=\omega_{N}^{-1}$ as characteristic scales it follows 


$$
C_{Y}\left(t^{*}\right)=a_{1}\left(\frac{Y^{\prime}}{U^{*}}\right)+a_{3}\left(\frac{Y^{\prime}}{U^{*}}\right)^{3}-\frac{a_{1}}{K U^{* 3}} Y^{\prime \prime}+\frac{a_{1}}{2 K^{2} U^{* 5}} Y^{\prime \prime \prime},
$$

where comma stands for differentiation with respect to the non-dimensional time. The dimensionless ODE equation for the dynamics of the body is now found

$$
Y^{\prime \prime}+2 \zeta Y^{\prime}+Y=\frac{U^{* 2}}{2 m^{*}}\left(a_{1}\left(\frac{Y^{\prime}}{U^{*}}\right)+a_{3}\left(\frac{Y^{\prime}}{U^{*}}\right)^{3}-\frac{a_{1}}{K U^{* 3}} Y^{\prime \prime}+\frac{a_{1}}{2 K^{2} U^{* 5}} Y^{\prime \prime \prime}\right)
$$

As can be seen, the Quasi-steady model is recovered when terms associated to time delay go to zero. At this point, it is convenient to summarize all the hypothesis introduced to the model:

- The fluid force coefficient at time $t$ is given by its quasi-steady value evaluated at the motion-induced angle of attack at time $t-\tau$, being $\tau$ a time delay.

- The time delay is a function of reduced velocities $V^{*}$ and $W^{*}=A^{*}$. A monomial relationship, $\tau^{*}=\gamma V^{* N} A^{* M}$, is assumed.

- It is assumed that $\gamma=1, A^{*} \approx C V^{*}, M=N=-1$, and $\omega^{* 2} \approx\left(1+C_{M} / m^{*}\right)^{-1}$, which gives $\tau^{*}=K^{-1} U^{*-2}$. Note this is a very crude approximation and it is expected not to work absolutely at low values of reduced velocity.

And the simplifications made:

- Approximation of $C_{Y Q S}(t-\tau)$ by a polynomial up to order $\tau^{3}$.

- Non-linear terms in $\tau$ and $\tau^{2}$ has not been considered for $C_{Y}(t)$.

\section{On the reduced velocity at which Galloping starts}

Oscillations start when total linear damping is zero. For sinusoidal oscillations $Y^{\prime \prime \prime} \approx-\omega^{* 2} Y^{\prime}$ and therefore, taking this approximation into account, total linear damping resulting zero in Eq. 3.16 means that

$$
4 m^{*} \zeta=a_{1} U^{*}-\frac{a_{1} \omega^{* 2}}{2 K^{2} U^{* 3}}
$$

If $m^{*} \zeta$ tends to zero, which is a normal condition in hydroelastic experiments $\left(m^{*}\right.$ of order unity and small $\zeta$ ) it follows that Galloping would start at the reduced velocity $U_{0}^{*}$ at which

$$
2 U_{0}^{* 4}-\frac{\omega_{0}^{* 2}}{K^{2}}=0
$$


If one consider, at first approximation $\omega_{0}^{*}=1$, then $U_{0}^{*}=\left(2 K^{2}\right)^{-1 / 4}$ which is a very different result than that predicted by the Quasi-steady model $\left(U_{0}^{*}=0\right.$; see Eq. 3.6). For large values of $m^{*} \zeta$, which is a usual condition in experiments in air $U_{0}^{*}$ tends to $4 m^{*} \zeta / a_{1}$.

\subsection{Validation of the model}

Two different validations are presented. First, a validation against free vibration experiments is considered. Later, forced oscillation experiments of a square section prism in an airstream from Bearman and Luo $(1988,1990)$ are considered. The model needs empirical information of the aerodynamic characteristics of the cross-section at hand. Fig. 3.1 shows experimental data for the transverse fluid force coefficient dependence with the angle of attack for square, rectangular, and triangular cross-sections. In addition, the cubic polynomial approximation considered $\left(a_{1}\right.$ and $\left.a_{3}\right)$ is shown.
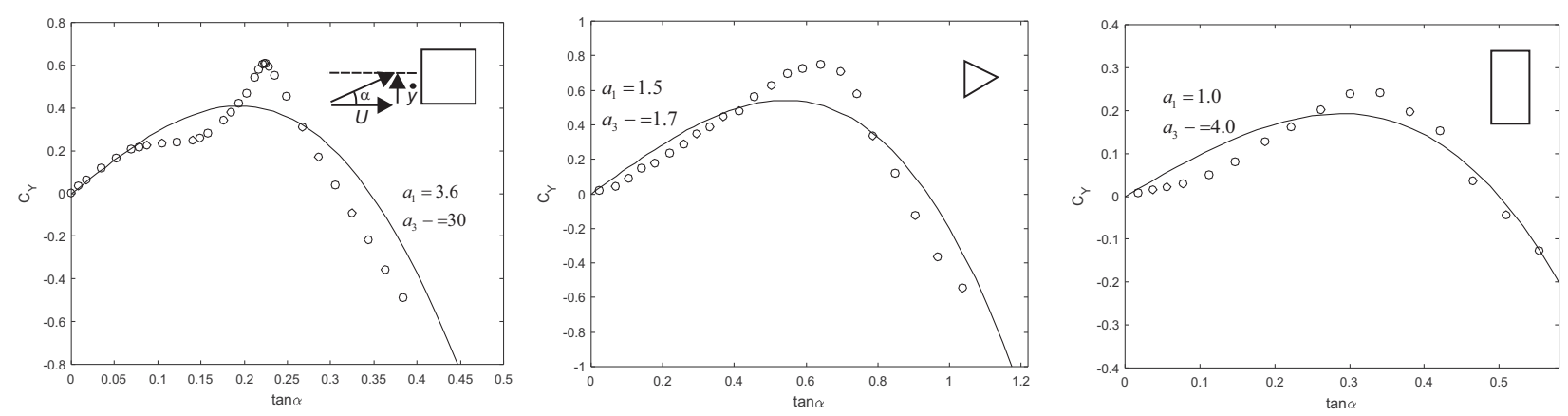

Figure 3.1: Variation of $C_{Y}$ with $\tan \alpha$ for square section from Bouclin and Parkinson (1977); for triangular and rectangular sections from Bokaian and Geeola (1983).

\subsubsection{Free vibration experiments. Analytical approach}

In order to obtain an analytical approach of the dynamics, a steady state of oscillations $Y=$ $A^{*} \sin \left(\omega^{*} t^{*}\right)$ is considered. After substituting the above mentioned expression along with $Y^{\prime}$ and $Y^{\prime \prime}$ in Eq. 3.17, and equate sine and cosine terms, one obtains

$$
\begin{gathered}
\left(1+\frac{a_{1}}{2 m^{*} U^{*} K}\right) \omega^{* 2}-1=0 \\
A^{*}=\frac{1}{\omega^{*}}\left(\frac{4 U^{*}}{3 a_{3}}\left(4 m^{*} \zeta-a_{1} U^{*}\left(1-\frac{\omega^{* 2}}{2 K^{2} U^{* 4}}\right)\right)\right)^{1 / 2},
\end{gathered}
$$

from which

$$
\omega^{*}=\left(1+\frac{a_{1}}{2 m^{*} U^{*} K}\right)^{-1 / 2}
$$


and

$$
A^{*}=C U^{*} F
$$

with

$$
F=\left(\left(1+\frac{a_{1}}{2 m^{*} U^{*} K}\right)\left(1-\frac{4 m^{*} \zeta}{a_{1} U^{*}}-\left(\frac{1}{1+\frac{a_{1}}{2 m^{*} U^{*} K}}\right) \frac{1}{2 K^{2} U^{* 4}}\right)\right)^{1 / 2}
$$

This result explains some differences found in the relationship between $A^{*}$ and $U^{*}$ between Nemes et al. (2012), and Bouclin and Parkinson (carried out at larger values of $m^{*}$ ). In both cases a quasi-linear trend is observed but in Bouclin and Parkinson's experiments the constant of proportionality seems to be lower (Fig. 3.2). Eq. 3.24 indicates the sense of this point since the mass ratio in Nemes' experiments were significantly lower.

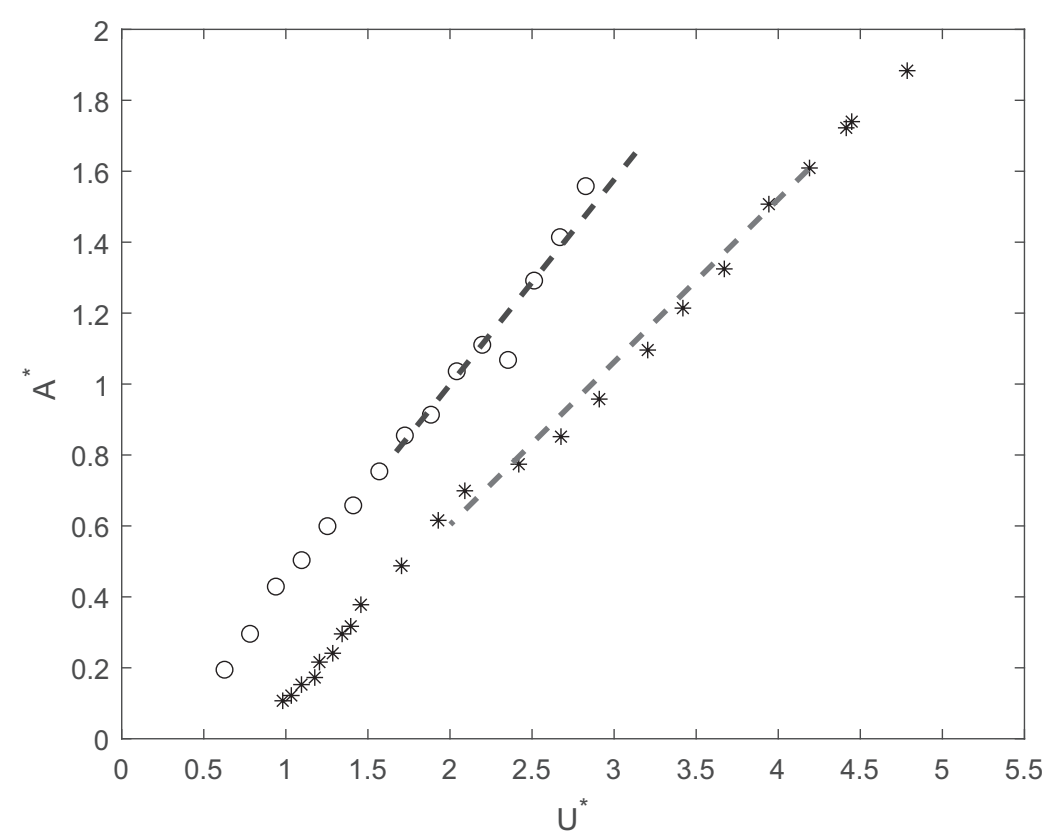

Figure 3.2: Normalized steady-state amplitude of oscillations variation with reduced velocity in Nemes at al. (2012), white dots; and Bouclin and Parkinson (1977), filled squares.

Fig. 3.3 shows a contour plot of $F$ with respect to $U^{*}$ and $m^{*}$ that would result for a square cross-section $\left(a_{1}=3.6, C=0.4\right.$, see Fig. 3.1, $\left.C_{M}=1.2\right)$ and negligible (zero) damping. As can be noted, for low values of mass ratio, $F$ may take values higher than the unity, which is the value predicted by the Quasi-steady model. As $U^{*}$ and $m^{*}$ grows $F$ approaches asymptotically to one. This result helps to explain why Bouclin and Parkinson experimental results gave a lower constant of proportionality $(C F)$ between amplitude of oscillations and reduced velocity in the linear trend regime.

$C$ and $a_{1}$ do not change qualitatively the behavior of $F$; lower values of $C$ increase the value of $F(\partial F / \partial C>0)$ and lower values of $a_{1}$ make $F$ get closer to one in a larger region of $U^{*}$ and $m^{*}\left(\partial F / \partial a_{1}<0\right)$.

Galloping starts when $A^{*}=0$, i.e when $F=0$, so now it is possible to give a better 


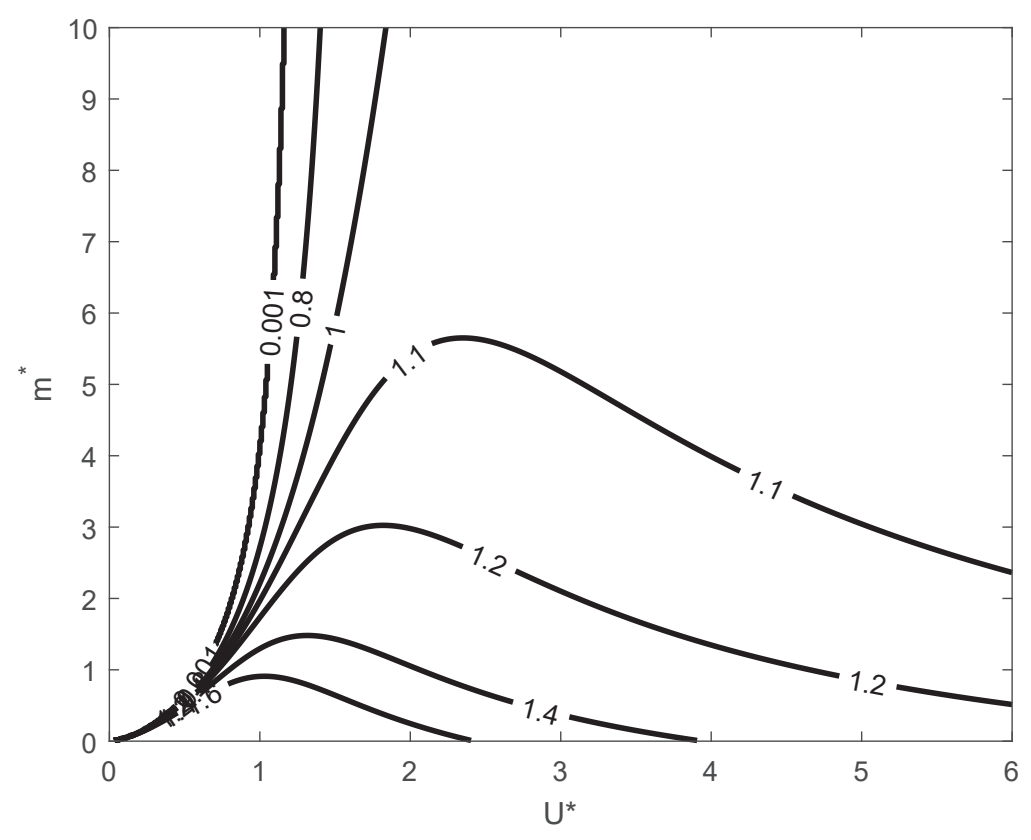

Figure 3.3: $F$ variation with the reduced velocity and the mass ratio. $\zeta=0$.

approximation that the one introduced earlier (where it was considered that $\omega_{0}^{*}=1$ ). For cases when $m^{*} \zeta \ll 1$, solving approximately, $F=0$ gives

$$
U_{0}^{*} \simeq\left(\frac{1}{2 K^{2}}\right)^{1 / 4}\left(\frac{1}{1+\frac{a_{1}}{m^{*}(2 K)^{3 / 4}}}\right)^{1 / 4},
$$

which indicates that $U_{0}^{*}$ diminishes as $m^{*}$ gets lower, as observed experimentally. Fig. 3.4 shows the variation with $m^{*}$ of the reduced velocity at which oscillations starts. Solid line represents the computed value from Eq. 3.24 for a square section (where $\zeta=0$ was taken), open circles stand for $\mathrm{Xu}-\mathrm{Xu}$ et al. (2016b) results (Chapter 2), and open squares results from Bouclin and Parkinson (1977), stars and filled squares stand for, respectively, results from Zhao et al. (2014) and Nemes et al. (2012). As can be seen, the model slightly oversize the value of $U_{0}^{*}$.

For low or moderate values of $m^{*}$ (as in hydroelastic situations), the frequency of oscillations may depart significantly from the unity. As can be seen in Eq. 3.23, the frequency of oscillations $\omega^{*}$ grows with reduced velocity to a saturation value of 1 . The lower $m^{*}$ is the higher values of reduced velocity are needed to be close to the saturation value of $\omega^{*}=1$.

Finally it can be seen that $\partial \omega^{*} / \partial C>0$ and $\partial A^{*} / \partial C<0$. This last result was anticipated by the definition of the time delay (Eq. 3.12), since it gets smaller as $C$ is higher. In other words, as $C$ is higher unsteady effects become less important and the dynamics are closer to the prediction of the Quasi-steady model. 


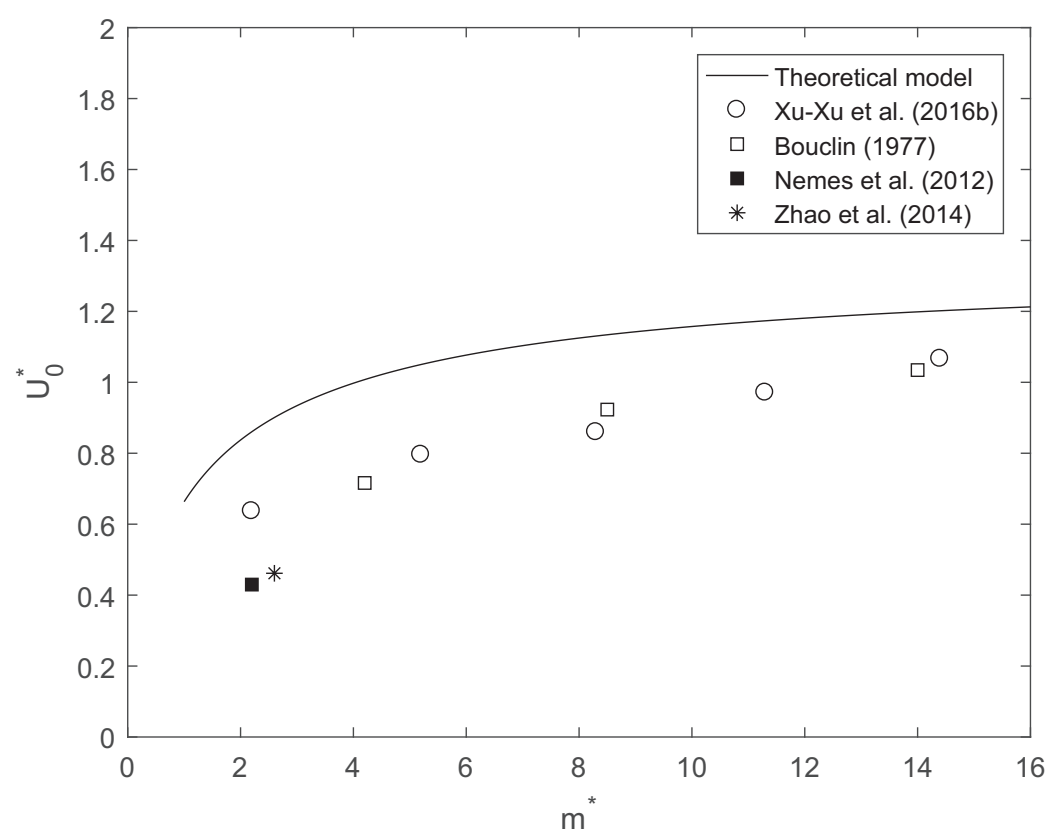

Figure 3.4: Variation of $U_{0}^{*}$ with $m^{*}$.

\section{On the Quasi-steady criterion}

A criterion for quasi-steady conditions can be introduced now in terms of the phase angle between the fluid forces and displacements, which is given by

$$
\phi=\tan ^{-1}\left(\frac{2 \zeta \omega^{*}}{1-\omega^{* 2}}\right)
$$

Taking into account Eq. 3.21 it follows that

$$
\phi=\tan ^{-1} \frac{2 \zeta\left(\frac{1}{1+\frac{a_{1}}{2 m^{*} U^{*}}}\right)^{1 / 2}}{1-\frac{1}{1-\frac{a_{1}}{2 m^{*} U^{*} K}}} .
$$

Fig. 3.5 shows phase angle measured by Bearman et al. (1987) for a square cylinder in free vibration experiments in air $\left(m^{*}=1013, \zeta_{1}=0.0006, \zeta_{2}=0.0009\right)$, as well as the prediction given by the theoretical model in Eq. 3.26. As can be seen, the model is able to capture experimental trends.

Under quasi-steady conditions $\phi$ has to be very close to $90^{\circ}$, so it has been proposed that quasi-steady conditions can be assumed if $\phi>85^{\circ}$, and therefore from Eq. 3.26 it follows that 


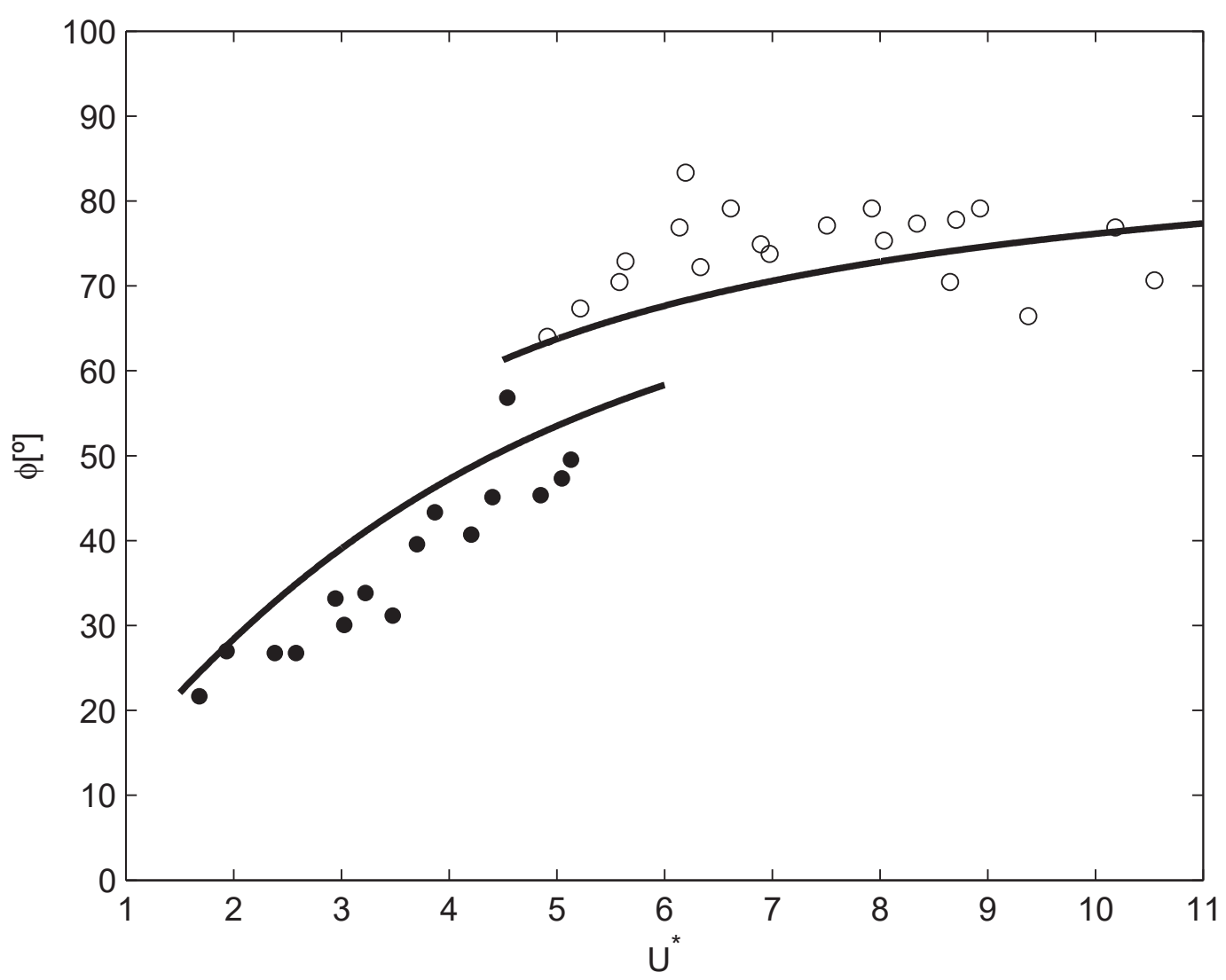

Figure 3.5: Phase angle from Bearman et al. (1987) experiments. $m^{*}=1013$ and $\zeta=0.0006$ (black circles) and $\zeta=0.0009$ (white circles). Solid is given by Eq. 3.26.

$$
\frac{2 \zeta\left(\frac{1}{1+\frac{a_{1}}{2 m^{*} U^{*} K}}\right)^{1 / 2}}{1-\frac{1}{1-\frac{a_{1}}{2 m^{*} U^{*} K}}}>\frac{3}{2} .
$$

An approximation, more useful from the practical side, is

$$
8 m^{*} \zeta U^{*} K>3 a_{1},
$$

which is proposed here as a new criterion to determine if quasi-steady conditions can be resorted to:

$$
\frac{8\left(m^{*}+C_{M}\right) \zeta U^{*}}{\left(-9 a_{1} a_{3} / 4\right)^{1 / 2}}>3
$$

since $K=\left(-4 a_{1} / 3 a_{3}\right)^{1 / 2}\left(1+C_{M} / m^{*}\right)$.

Eq. 3.29 helps to explain the reason why very large reduced velocities are needed to achieve 
full quasi-steady condition in hydroelastic situations ( $m^{*}$ of order unity) and low damping. In addition it shows why it is difficult to find a universal criteria for quasi-steady conditions using only the reduced velocity $U^{*}$, mechanical properties (through the mass-damping parameter) and geometry ( $a_{1}$ and $a_{3}$ play a role).

\section{Square cross-section. Model prediction and experimental results.}

A comparison of experimental results by Nemes et al. (2012), Zhao et al. (2014) as well as $\mathrm{Xu}-\mathrm{Xu}$ et al. (2016b) is made against results predicted by the theoretical model (Eqs. 3.20 and 3.21). In all cases, experiments were carried out with a elastically-mounted rigid square prism in a free-surface recirculating water channel in such a way that $m^{*}$ was in the range of 2.2-2.6.
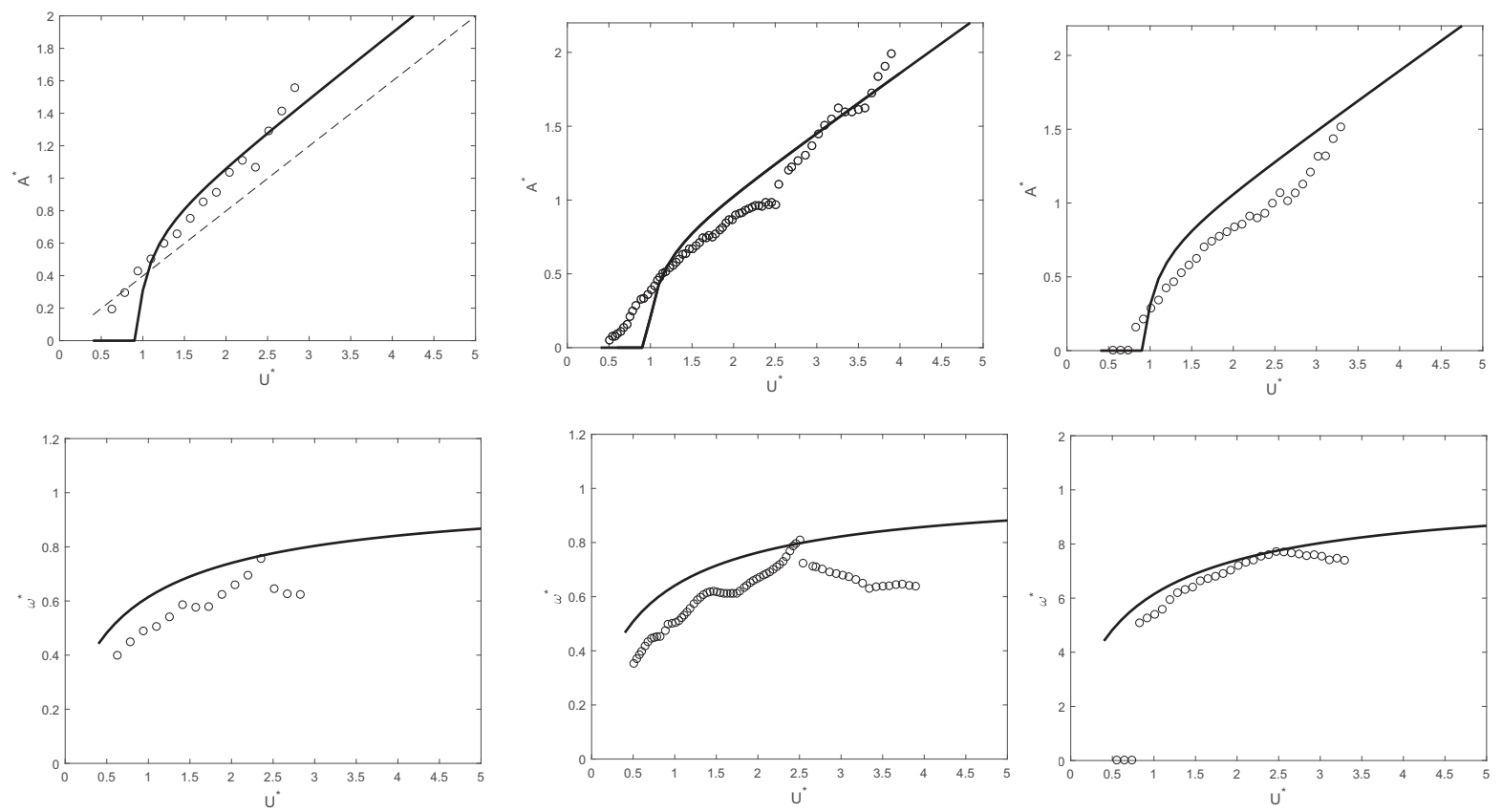

Figure 3.6: $A^{*}$ and $f^{*}$ variation with reduced velocity $U^{*}$. From left to the right: Nemes et al. (2012), Zhao et al. (2014) and Xu-Xu et al. (2016b). Model prediction in solid line.

As can be observed in Fig. 3.6, the overall agreement is good. From experiments (white circles), it may be observed that the frequency of oscillations has a more complex dependence with the reduced velocity and the simplifications introduced in the theoretical model, such as describing the $C_{Y}$ dependence with $\tan (\alpha)$ only with a cubic polynomial, or retaining only linear terms in $\tau$ and $\tau^{2}$ (first damping and stiffness corrections), may explain the discrepancies. Also, the velocity at which oscillations start is slightly oversized.

In Fig. 3.6 (NW corner) it is also shown (in dashed line) amplitude of oscillations predicted by the Quasi-steady model. It can be seen that amplitude of oscillations are underestimated in Quasi-steady model. This under-estimation is expected to be larger as $m^{*}$ gets lower. 


\section{Other cross-sections. Model prediction and experimental results}

To test the robustness of the proposed theoretical model, comparison is made now with other geometries. Theoretical predictions are shown in Fig. 3.7 for a rectangular $\left(m^{*}=8.5, \zeta=\right.$ $0.019)$ and a triangular cross-section $\left(m^{*}=14.5, \zeta=0.014\right)$ as well as experimental results found by Bokaian and Geeola (1983) in hydroelastic tests. Static dependence of $C_{Y}$ with the angle of attack for these geometries is shown in Fig. 3.1.
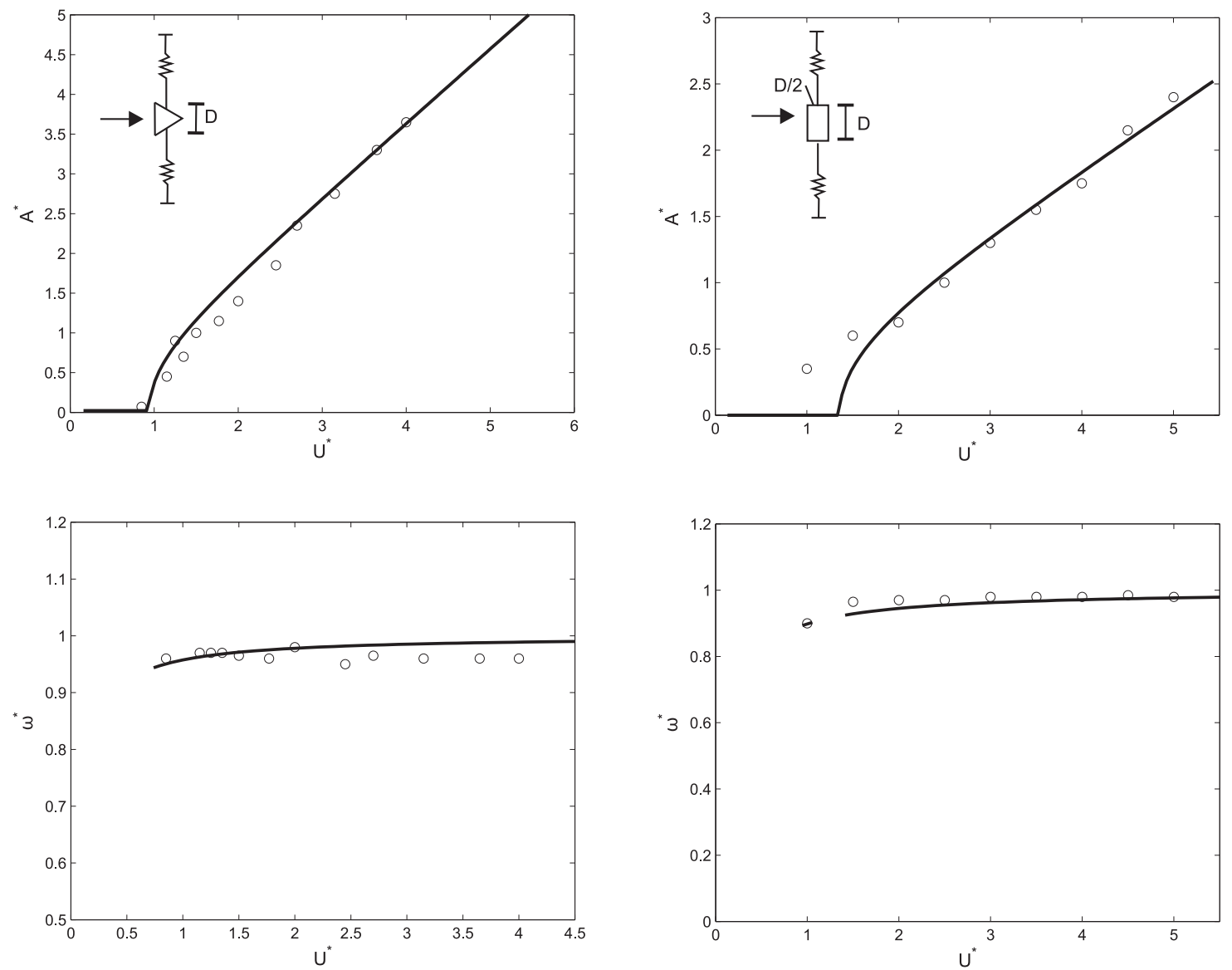

Figure 3.7: $A^{*}$ and $f^{*}$ variation with reduced velocity $U^{*}$ for isosceles triangular and rectangular cross section. Model prediction in solid line.

\subsubsection{Forced vibrations of a square cylinder}

Bearman and Luo $(1988,1990)$ conducted controlled forced oscillation experiments on a square section prism in an airstream. They made measurements of fluid forces on the oscillating prisms by averaging instantaneous pressure distributions on the surface. The ranges of normalized amplitude of oscillations and reduced velocities were $A^{*}=0.5,0.675,1,1.5,2$ and $V^{*}=U /(\omega D)=U^{*} / \omega^{*}=0-40$. Fluid force measurements were given in terms of harmonic components of lift coefficient deduced from a Fourier analysis of pressure measurements, 
and a phase angle by which fluid force lead displacements. They found useful to introduce a new parameter $V^{*} / A^{*}=U^{*} /\left(A^{*} \omega^{*}\right)=1 / \lambda^{*}$ and present fluid forces dependence with this parameter.

During each forced experiment the oscillations had a harmonic aspect with a normalized amplitude $A^{*}: Y=A^{*} \sin \left(t^{*}\right)$. Then, from Eq. 3.15

$$
C_{Y}\left(t^{*}\right)=\left(a_{1}\left(\lambda^{*}-\frac{\lambda^{* 5}}{2 A^{* 2} K^{4}}\right)+\frac{3 a_{3}}{4} \lambda^{* 3}\right) \cos \left(t^{*}\right)+\left(\frac{a_{1} \lambda^{* 3}}{A^{* 2} K}\right) \sin \left(t^{*}\right) .
$$

The phase angle $\phi$ by which force leads displacements is therefore given by

$$
\phi=\tan ^{-1}\left(\frac{A^{* 2} K\left(a_{1}\left(\lambda^{*}-\frac{\lambda^{* 5}}{2 A^{* 2} K^{5}}\right)+\frac{3 a_{3}}{4} \lambda^{* 3}\right)}{a_{1} \lambda^{* 3}}\right),
$$

which indicates that $\phi$ depends on both $A^{*}$ and $\lambda^{*}$. Module of $C_{Y}$ can also be computed from Eq. 3.31

$$
\left|C_{Y}\right|=\left(\left(a_{1}\left(\lambda^{*}-\frac{\lambda^{* 5}}{2 A^{* 2} K^{4}}\right)+\frac{3 a_{3}}{4} \lambda^{* 3}\right)^{2}+\left(\frac{a_{1} \lambda^{* 3}}{A^{* 2} K}\right)^{2}\right)^{1 / 2}
$$

Fig. 3.8 shows Luo and Bearman (1988) measurements for the first harmonic component of transverse fluid force $\left(C_{L}\right)$ and phase angle $\phi$, as well as the theoretical results predicted by Eqs. 3.31 and 3.32. For the square section $C=0.4$ (see Fig 3.1, and note that for forced vibrations $\left.m^{*}=\infty\right)$. As can be seen the agreement is good. As the amplitude of oscillations increases, quasi-steady conditions are found earlier (lower values of $1 / \lambda^{*}$ ), that is $\phi$ close to $90^{\circ}$,
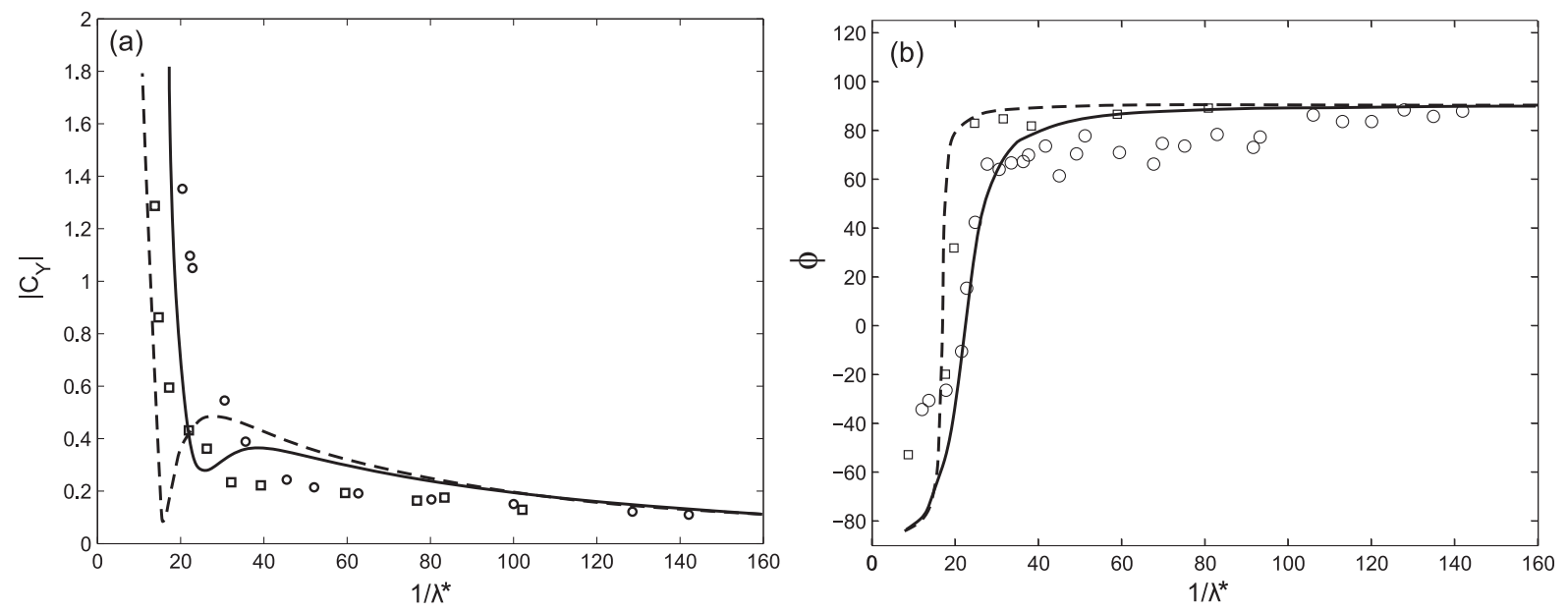

Figure 3.8: Fluid force coefficient as a function of $\lambda^{*-1} . A^{*}=0.675$ (exp: circles; model: dashed line) and $A^{*}=2$ (exp: squares; model: solid line). 


\subsection{Concluding remarks}

The aim of this development has been to improve the phenomenological modelling of transverseinduced vibrations of an elastically mounted non-circular bluff body in fluid flow. A time delayed theoretical Quasi-steady model has been developed. The theoretical model is based on the introduction of a time delay which is only function of the static empirical coefficients used in the traditional Quasi-steady model. The model dynamics is investigated analytically and closed-form relations of the model response are derived and compared to previous experimental results (both in free and forced vibrations). Experimental results agree well with those given by the theoretical model qualitatively, and to a certain extent quantitatively. In addition, the model helps to gain insight about experimental results not explained satisfactory by the Quasi-steady theory, such as:

- The frequency of oscillations depends strongly on $m^{*}$. Because of the time delay, fluid inertia force effects may be explained by a time delay effect which is more important for low reduced velocities.

- There are two different behaviours (branches) in the dependence of the steady-state normalized amplitude of oscillations $A^{*}$ with the reduced velocity. At the initial stage, called here initial branch, $A^{*} \propto\left(U^{*}\right)^{N}$ with $1 / 2<N<1$. $N$ grows with $U^{*}$ until reaches the value of unity (linear branch). At the linear branch the constant of proportionality between $A^{*}$ and $U^{*}$ depends on the mass ratio.

- The reduced velocity at which oscillations start $U_{0}^{*}$, which can occur below the reduced velocity at which the resonance condition for Vortex Induced Vibrations takes place. In other words, oscillations may start at reduced velocities significantly lower than $S t^{-1}$, where $S t$ is the stationary Strouhal number. In addition the model predicted $U_{0}^{*}$ is mainly driven by $m^{*}$ and cross-section geometry (see Eq. 3.24) and not by the mass damping parameter $m^{*} \zeta$ as proposed by the Quasi-steady model.

- The criterion for quasi-steady conditions cannot be fixed only by the reduced velocity $U^{*}$ (say, higher than a certain fixed threshold). The model has helped to propose a criterion where the threshold for the reduced velocity depends on the cross-section geometry, mass ratio and damping. This may explain the lack of agreement observed in the literature to establish a criterion.

- In forced vibrations tests, according to the model, fluid forces depend on both $V^{*}$ and $A^{*}$ and is not possible to describe them with a single parameter $V^{*} / A^{*}$.

In addition, the model can be useful

- for designing purposes, to estimate when Galloping is expected to start and the magnitude of oscillations. Because of the simplicity of the model, it requires little time to compute results. In addition it requires not very much experimental information (static) as an input. As known, static tests are easy to perform in comparison with unsteady tests. This is one of the main advantages of the phenomenological approach of the model. 
- to design numerical experiments for studying specific questions. For example to see more clearly the dependence of the constant of proportionality between $A^{*}$ and $U^{*}$ in the linear range on the mass ratio, where very low mass ratio experiments should be carried out (say, $m^{*}<1$ ); or to make direct comparison between fluid-force and quasi-steady fluid force.

- to design PIV experiments or computational experiments in order to see if there appears time-averaged wake lags with the motion of the body in the way as purposed in the model.

However the model presents some weak points. For example, from a dimensional analysis perspective one expect that the time delay should have the following dependence:

$$
\tau=\tau\left(U, D, \omega_{N}, \dot{y}, \ddot{y}\right)
$$

so that if $D$ and $\omega_{N}$ are taken as characteristic scales one have

$$
\tau^{*}=\tau \omega_{N}=\tau^{*}\left(U^{*}, \dot{y} /\left(\omega_{N} D\right), \ddot{y} /\left(\omega_{N}^{2} D\right)\right) .
$$

However, it was taken time averaged quantities of $\dot{y} /\left(\omega_{N} D\right)$, and $\ddot{y} /\left(\omega_{N}^{2} D\right)$ and therefore $\tau^{*}=\tau^{*}\left(U^{*}, A^{*}, \omega^{*}\right)$. This step is not based on clear physical reasons. It was assumed that instead of a time delay changing continuously with time, the time delay is fixed for a certain true reduced velocity $U^{*} / \omega^{*}$ and amplitude of oscillations $A^{*}$. This behaviour does not appear too realistic and one would rather expect a continuous evolution of the fluid force. Also, is was considered that $\tau^{*} \propto\left(U^{*} A^{*}\right)^{-1}$ in a rather speculative way. A physical reason between the proposed law for the time delay has not been found yet. Finally, it should be stressed that from the quantitative point of view there is two basic simplifications introduced in the model: (i) $C_{Y}$ dependence with $\alpha$ has considered only a cubic polynomial, (ii) only linear term in $\tau$ and $\tau^{2}$ has been retained. In addition to that it is clear that the $C_{Y}$ static curve depends on a large number of parameters in addition to the cross-section geometry (eg. inflow conditions: turbulence, non-uniformity). 


\section{Chapter 4}

\section{Energy harvesting of a cantilever-mounted prism}

\subsection{Introduction}

Flow-induced oscillations by Transverse Galloping (TG) were pointed out by Barrero-Gil et al. (2010) as a potential source for energy harvesting from an airstream. TG is a fluidelastic instability that appears in some elastic bluff bodies when the velocity of the incident flow exceeds a critical value. Then, oscillatory motion (transverse to the flow) develops with increasing amplitude until the energy dissipated per cycle by mechanical damping balances the energy input per cycle from the flow (for a detailed introduction to TG the reader is referred to Parkinson 1989, or Paidoussis et al. 2011). If the geometry of the body and the elastic properties are appropriate, the TG instability may appear at low flow velocities and with large excitation amplitudes, making TG a very promising way to harvest energy successfully (Barrero-Gil et al. 2010, Vicente-Ludlam et al. 2014, 2015).

Barrero-Gil et al. (2010) made an analytical treatment to give the level of mechanical power extraction as a function of the geometry of the cross-section of the galloping body, its mechanical parameters, and flow velocity. Findings like the maximum efficiency achievable or the wind speed at which this maximum occurs were reported. Since then, several researches have studied how to implement the concept in a real energy harvester, with emphasis in low power generation systems, of the order of milli-Watts or tens of milli-Watts, [see, for example, Sirohi and Mahadik (2011, 2012), Zhao et al. (2013), Yang et al. (2013), Xu-Xu et al. (2015)], with characteristic dimensions of the order of centimetres. They have been focused on experimental arrangements where a rigid galloping body is fixed to the free end of a cantilevered beam (see Fig. 4.1). For electricity conversion piezoelectric sheets are usually attached to the base of the beam. Under the effect of an airstream, for high enough air speed, oscillations by Galloping take place and the induced strain in the piezoelectric patches produces an electrical current which is dissipated at the electrical load $R_{L}$ (see, for example, Yang et al. 2013). However, in this cantilevered arrangement the situation is not entirely analogous to that of pure TG analysed in Barrero-Gil et al. (2010), since the instantaneous attitude of the galloping body with respect to the incident flow depends on the velocity of galloping body and wind speed (like in TG) but also on the rotation angle at the beam free end (see Fig. 4.1; Kluger et al. 2013). A new governing parameter appears, namely the ratio of the cross-section characteristic length $D$ to the cantilever beam length $L_{b}$, defined as $\delta=3 D /\left(2 L_{b}\right)$, and its role on the dynamics of the body and electrical power output should be studied. With this idea in mind, a theoretical model of a generic energy harvester is presented here where the galloping body is cantilevered mounted. Quasi-steady conditions are assumed to model aerodynamic forces and a kinematic relationship is introduced for the instantaneous angle of attack where rotation of the beam is considered. An equivalent circuit model is employed for the piezoelectric sheets. The mathematical model 
is approximately solved by applying the standard Harmonic Balance Method, and analysed in detail. The analysis is focused on the impact of the system dimensionless parameters on the efficiency of energy harvested. As a novelty, the analysis allows to see clearly that $\delta$ has a negative impact into the electrical power output.

(a)

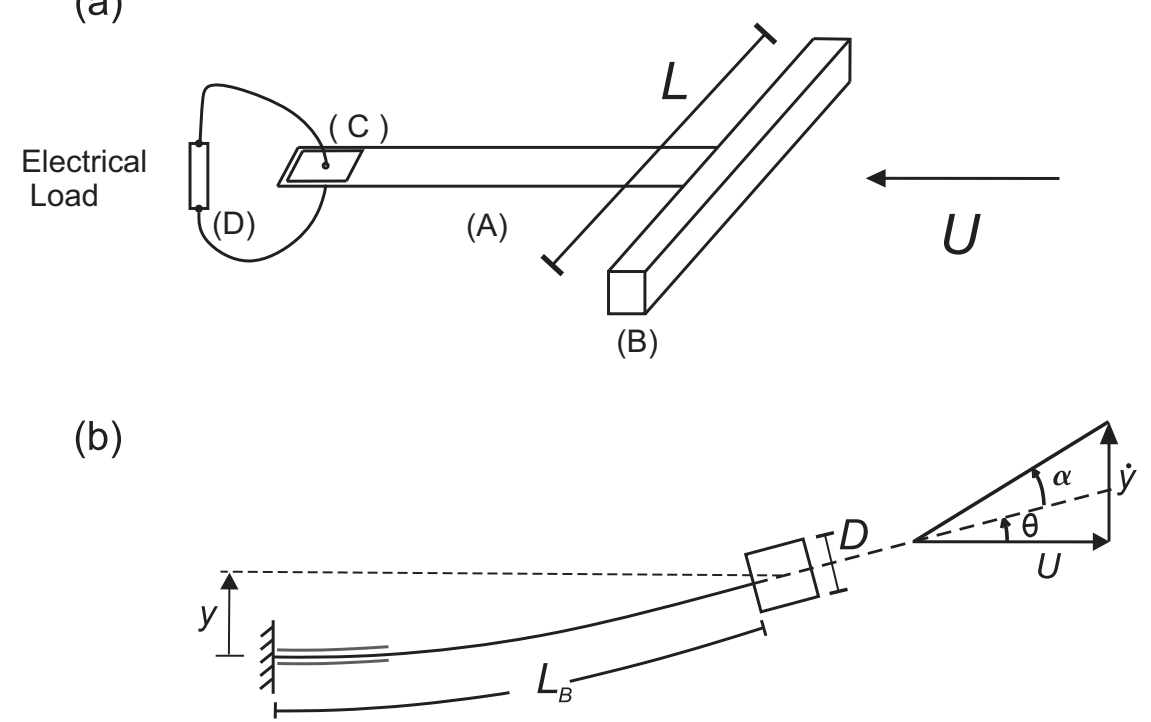

Figure 4.1: (a) Typical arrangement piezoelectric energy harvesting from Galloping of a cantilevered prism. (b) One-degree-of-freedom model: vertical displacement of the rigid bluff body.

First of all, in Section 4.2, an electro-aero-elastic model is introduced. An analytical approximate solution is found in Section 4.3 that allows to get physical insight and to discuss the influence of the length of the beam and other governing parameters on both the galloping body dynamics and electrical power. Analytical predictions are compared with experimental results from Zhao et al. (2013) in Section 4.4. Good agreement is found. Finally, concluding remarks are drawn in Section 4.5.

\subsection{Theoretical model}

One-degree-of-freedom model is introduced in order to describe the transverse displacement of the prism shown in Fig. 4.1. It is based on the equilibrium between inertia, damping, and stiffness forces, as well as vertical aerodynamic force, and the electromechanical force induced by the piezoelectric transducer. That is,

$$
m \ddot{y}+c \dot{y}+K y=\frac{1}{2} \rho U^{2} D L C_{Y}-F_{p} .
$$

where $y$ denotes the transverse position of the prism, $m$ is the equivalent mass of the prism, $c$ is an equivalent damping constant, $k$ is the equivalent stiffness constant, $\rho$ is the fluid density, $U$ is the undisturbed velocity of the incident flow, $D$ the side length of the prism's cross-section and $L$ its length, $C_{Y}$ is the instantaneous aerodynamic force coefficient in the transverse direction to 
the incident flow, and $F_{p}$ is the electromechanical force in the $y$ direction due to the piezoelectric effect. Finally, the dot symbol stands for differentiation with respect to physical time $t$.

The equivalent (or effective) mass of the prism is given by the prism mass plus the effective mass of cantilever beam. The effective mass of cantilever beam can be approximated as 0.25 times the mass of the cantilever beam (see Virgin, 2000). The equivalent damping and stiffness constants can be obtained experimentally from a free decay tests in absence of fluid flow by measuring the decay rate of the amplitude and frequency of oscillations.

Note that, for the sake of simplicity, damping and stiffness forces have been considered linear, which is a realistic approximation when transverse displacements of the prism are small compared to the length of the beam.

\subsubsection{Aerodynamic force}

In order to describe CY, the Quasi-steady hypothesis is usually resorted to (see Paidoussis et al. 2011), since Galloping is typically a low-frequency oscillation phenomenon where the characteristic timescale of the prism oscillation (of order $2 \pi(m / k)^{1 / 2}$ ) is much larger than the characteristic timescale of the flow (of order $D / U$ ). Then, the aerodynamic force is only dependent on the instantaneous attitude of the prism with respect to the incident flow, which can be described by the effective angle of attack $\alpha$. From Fig. 4.1b,

$$
\tan (\alpha+\theta)=\frac{\tan \alpha+\tan \theta}{1-\tan \alpha \tan \theta}
$$

where $\theta$ is the rotation angle at the free end of the beam; $\alpha$ and $\theta$ are positive in the counterclockwise direction. Assuming that $\alpha$ and $\theta$ are small it follows that

$$
\tan (\alpha+\theta) \simeq \tan \alpha+\tan \theta
$$

For a uniform cantilevered beam, $\theta=3 y /\left(2 L_{b}\right)$ (see Kluger et al. 2013), where $L_{b}$ is the length of the beam. In addition, $\tan (\alpha+\theta)=\dot{y} / U$ so it follows that

$$
\tan \alpha \simeq \frac{\dot{y}}{U}-\frac{3 y}{2 L_{b}}
$$

For this theoretical analysis, to maintain a compromise between development complexity and accuracy, a cubic polynomial can be considered enough (see Blevins, 1990 [p. 130]) to approximate the vertical aerodynamic force coefficient dependence with $\tan \alpha$, so that

$$
C_{Y}=a_{1} \tan \alpha+a_{3} \tan ^{3} \alpha
$$

where $a_{1}(>0)$ and $a_{3}(<0)$ are the empirical coefficients to fit by a polynomial the $C_{Y}$ versus $\tan (\alpha)$ dependence measured in static tests (normally in wind tunnel). The values of $a_{1}$ and $a_{3}$ depend on the cross-section geometry of the prism. The reader is referred to Blevins (1990), 
Bokaian and Geoola (1983) or Barrero-Gil et al. (2010) in order to obtain more information and typical values. Then, the aerodynamic force coefficient is

$$
C_{Y}=a_{1}\left(\frac{\dot{(y)}}{U}-\frac{3 y}{2 L_{b}}\right)+a_{3}\left(\frac{\dot{(y)}}{U}-\frac{3 y}{2 L_{b}}\right)^{3},
$$

which can be simplified to

$$
C_{Y}=a_{1}\left(\frac{\dot{(y)}}{U}-\frac{3 y}{2 L_{b}}\right)+a_{3}\left(\left(\frac{\dot{(y)}}{U}\right)^{3}+\frac{27 y^{2} \dot{(y)}}{4 L_{b}^{2} U}\right),
$$

if nonlinear stiffness terms are neglected, which makes sense since their effect in the overall response is expected to be small when the bluff body is under the action of light fluids (airstreams). Discussing this point in detail, the nonlinear stiffness fluid force $\tilde{F}_{y}$ has been compared

$$
\tilde{F}_{y}=\frac{1}{2} \rho U^{2} D L\left(\frac{27 y^{3}}{8 L_{b}^{3}}-\frac{9 \dot{y}^{2} y}{2 U^{2} L_{b}}\right)
$$

and the stiffness force $F_{s}=K y$. That is

$$
\frac{\tilde{F}_{y}}{F_{s}}=\frac{\rho U^{2} D L}{2 K}\left(\frac{27 y^{3}}{8 L_{b}^{3}}-\frac{9 \dot{y}^{2} y}{2 U^{2} L_{b}}\right) .
$$

Taking $y \sim A$, where $A$ is the steady-state amplitude of oscillations, $(y) \sim A \omega_{N}$ where $\omega_{N}^{2}=k / m$ is the natural frequency of oscillations, it follows that

$$
\frac{\tilde{F}_{y}}{F_{s}} \sim \frac{a_{3}}{2 m^{*}}\left(A^{* 2} \delta^{3} U^{* 2}-3 A^{* 2} \delta\right),
$$

where $m^{*}=m /\left(\rho D^{2} L\right)$ is the mass ratio, $A^{*}=A / D$ is the normalized steady state amplitude of oscillations, $\delta=3 D /\left(2 L_{b}\right)$, and $U^{*}=U /\left(\omega_{N} D\right)$ is the reduced velocity. Note that nonlinear stiffness fluid force terms are expected to be negligible for large $m^{*}$, which is a common situation when the fluid is light (airstreams for example).

\subsubsection{Piezoelectric force}

An equivalent circuit is usually employed to build a linear lumped-model of the piezoelectric sheet (Ertuk et al. 2010). Then

$$
C_{p} v^{\prime}+\frac{v}{R_{L}}+\dot{\Theta(y)}=0,
$$


and

$$
F_{p}=-\Theta v
$$

where $v$ is the voltage across the electrical resistance, $C_{p}$ is the equivalent capacitance of the piezoelectric sheets, $\Theta$ is an electromechanical coupling constant, and $R_{L}$ the electrical load resistance. Considering a steady-state of sinusoidal oscillations, that is

$$
y=A \sin \omega t
$$

and,

$$
v=v_{0} \sin (\omega t+\phi)
$$

where $A$ and $\omega$ are, respectively, the amplitude and frequency of oscillations, $v_{0}$ the voltage amplitude and $\phi$ the phase delay between voltage and transverse displacement of the beam. Substituting Eqs. 4.13 and 4.14 into Eq. 4.11 and equating sine and cosine terms it follows that

$$
v_{0} \sin \phi=-\frac{\Theta R_{L} A \omega}{1+C_{p}^{2} R_{L}^{2} \omega^{2}}
$$

and

$$
v_{0} \cos \phi=-C_{p} R_{L} \omega \frac{\Theta R_{L} A \omega}{1+C_{p}^{2} R_{L}^{2} \omega^{2}}
$$

Since $\dot{(} y)=A \omega \cos (\omega t)$ it follows from Eq.4.12 and Eqs. 4.15 and 4.16 that the piezoelectric force can be split into a damping term and a stiffness one

$$
F_{p}=c_{E} \dot{(y)}+c_{E} C_{p} R_{L} \omega^{2} y
$$

with

$$
c_{E}=\frac{\Theta^{2} R_{L}}{1+C_{p}^{2} R_{L}^{2} \omega^{2}} .
$$

\subsubsection{Dimensionless equation}

Taking into account Eqs. 4.7 and 4.17, Eq. 4.2 can now be rewritten in dimensionless form as

$$
Y^{\prime \prime}+2 \zeta Y^{\prime}+Y=\frac{U^{* 2} a_{1}}{2 m^{*}}\left(\frac{Y^{\prime}}{U^{*}}-\delta Y\right)+\frac{U^{* 2} a_{3}}{2 m^{*}}\left(\frac{Y^{\prime 3}}{U^{*}}-\frac{3 \delta^{2} Y^{2} Y^{\prime}}{U^{*}}\right)-2 \zeta_{E} Y^{\prime}-2 \zeta_{E} \beta \omega^{* 2} Y
$$


where comma stands differentiation with respect to the dimensionless time, $\tau=\omega_{N} t, Y=$ $y / D$ is the normalized displacement, $\zeta=c /\left(2 m \omega_{N}\right)$ is the dimensionless parasite mechanical damping, $m^{*}=m /\left(\rho D^{2} L\right)$ is the mass ratio, $\delta=3 D /\left(2 L_{b}\right), U^{*}=U /\left(\omega_{N} D\right)$ the reduced velocity, and $\zeta_{E}$ is a dimensionless piezoelectric damping coefficient, given by

$$
\zeta_{E}=\frac{\Theta^{2} R_{L}}{2 m \omega_{N}\left(1+C_{p}^{2} R_{L}^{2} \omega^{2}\right)}
$$

$\beta=R_{L} C_{p} \omega_{N}$ y $\omega^{*}=\omega / \omega_{N}$.

The last term of Eq. $4.19\left(2 \zeta_{E} \beta \omega^{* 2} Y\right)$ contributes to the stiffness component of the dynamic equation. When comparing the order of magnitude of this term with the stiffness leading term (of order unity), it is noted that it can be neglected in practical situations since $2 \zeta_{E} \beta \omega^{* 2} \ll 1$. $2 \zeta_{E} \beta \omega^{* 2} Y$ can be rewritten, taking into account the definition in Eq. 4.20, as:

$$
2 \zeta_{E} \beta \omega^{* 2}=\frac{\Theta^{2}}{m \omega_{N}^{2} C_{p}} \frac{\beta^{2} \omega^{* 2}}{1+\beta^{2} \omega^{* 2}} .
$$

The second term, $\beta^{2} \omega^{* 2} /\left(1+\beta^{2} \omega^{* 2}\right)$, is always under unity. Checking the first term $\frac{\Theta^{2}}{m \omega_{N}^{2} C_{p}}$, it can be seen that:

- The value of the electromechanical coupling constant $\Theta$ is dictated by the state of art of the piezoelectric sheets, and it is or the order $10^{-4} \mathrm{~N} / \mathrm{V}$ nowadays.

- $C_{p}$ is also dictated by the state of art of the piezoelectric sheets, it is about $10^{-7} \mathrm{~F}$ for commercial piezoelectric sheets.

- $m \omega_{N}^{2}$ is equal to the cantilever beam stiffness constant, which depends on the cantilever beam elastic properties and geometric characteristics (cross-section and length of the beam). It takes often a value of the order of $100 \mathrm{~N} / \mathrm{m}$ (see for example, experiments of Zhao et al. 2013).

These parameters above yield that $2 \zeta_{E} \beta \omega^{* 2} \sim 10^{-3}$. Based on that, the conclusion is, unless the stiffness of the cantilever beam is very low, that the last term of Eq. 4.19 can be neglected.

As can be seen in Eq. 4.19, at this level of approach, for $\delta \neq 0$ the aerodynamic force introduces into the dynamics of the prism an additional linear stiffness term as well as a nonlinear damping term. When $\delta \rightarrow 0$, Eq. 4.19 tends to that of pure TG (see Barrero-Gil et al. 2010).

\subsubsection{Electrical power}

The mean electrical power $P_{E}$ dissipated at the electrical load is given by, 


$$
P_{E}=\frac{1}{T} \int_{0}^{T} \frac{v^{2}}{R_{L}} \mathrm{dt} \simeq \frac{1}{T} \int_{0}^{T} \frac{\left(v_{0} \sin \Phi\right)^{2}}{R_{L}} \mathrm{dt}=\frac{1}{T} \int_{0}^{T} 2 m \omega_{N} \zeta_{E} \dot{y}^{2} \mathrm{dt},
$$

where it has been assumed that $v_{0} \cos \Phi \ll v_{0} \sin \Phi$ (note that $v_{0} \cos \Phi=R_{L} C_{p} \omega v_{0} \sin \Phi$ and for practical situations $R_{L} C_{p} \ll 1$ ); $T$ is a period time for averaging. An alternative way to arrive to the same result is to note that the power dissipated at the piezoelectric sheet is given by

$$
P_{E}=\frac{1}{T} \int_{0}^{T} F_{p} \dot{(y)} \mathrm{dt}=\frac{1}{T} \int_{0}^{T} c_{E} \dot{y}^{2} \mathrm{dt}=\frac{1}{T} \int_{0}^{T} c_{E} C_{p} R_{L} \omega^{2} \dot{y}(y) \mathrm{dt}
$$

When non-linearity in Eq. 4.19 is small (high $m^{*}$ which is usual in aero-elastic situations) a steady-state of sinusoidal oscillation is expected so that the last term of Eq. 4.23 disappears and

$$
P_{E}=\frac{1}{T} \int_{0}^{T} c_{E} \dot{y}^{2} \mathrm{dt}=\frac{1}{T} \int_{0}^{T} 2 m \omega_{N} \zeta_{E} \dot{y}^{2} \mathrm{dt},
$$

which coincides with the expression given in Eq. 4.22.

An efficiency factor $\eta$ can be introduced relating the mean electrical power dissipated with the total power in the flow per frontal area of the prism

$$
\eta=\frac{P_{E}}{\frac{1}{2} \rho U^{3} D L}
$$

\subsection{Analytical solution}

\subsubsection{Dynamic response}

Eq. 4.19 contains 7 dimensionless parameters $\left(m^{*}, \zeta, \zeta_{E}, U^{*}, \delta, a_{1}, a_{3}\right)$, meaning that the solution manifold exists inside a 7 -dimensional space. To gather a better understanding in this 7-dimensional space, the use of a theoretical analysis able to yield an analytical prediction, valid within the assumptions made, is deemed to be beneficial because it clearly identifies the role in the dynamics of any of the governing parameters. Eq. 4.19 allows an analytical approach when the nonlinear terms are small compared to the linear ones. That is when $m^{*} \gg 1$, which is a usual condition when the fluid medium is air. In this case, solution to the nonlinear Eq. 4.19 is close to that of its linear version and thus one may assume that the steady movement response is sinusoidal, $Y=A^{*} \sin \left(\omega^{*} \tau\right)$, where $\omega^{*}=\omega / \omega_{N}$ and $A^{*}=A / D$ being $A$ the amplitude of steady oscillations. In addition, if one takes into account that $\cos ^{3}\left(\omega^{*} \tau\right) \approx 3 \cos \left(\omega^{*} \tau\right) / 4$, (i.e. higher order terms in $\cos \left(3 \omega^{*} t\right)$ are neglected $)$, equating sine and cosine terms, after some algebra one gets 


$$
\begin{gathered}
\omega^{* 2}=1+\frac{a_{1} U^{* 2} \delta}{2 m^{*}}=k_{1}, \\
A^{* 2}=\frac{4}{3} \frac{1}{\frac{a_{3} \omega^{* 2}}{U^{*}}+U^{*} \delta^{2} a_{3}}\left(4 m^{*}\left(\zeta+\zeta_{E}\right)-a_{1} U^{*}\right),
\end{gathered}
$$

where $k_{1}$ is a parameter introduced for convenience. Eq. $4.26 \mathrm{~b}$ can be written in an explicit way if Eq. 4.26a is taken into account,

$$
A^{* 2}=\frac{4 U^{*}}{3 a_{3}}\left(4 m^{*}\left(\zeta+\zeta_{E}\right)-a_{1} U^{*}\right) k_{2},
$$

where $k_{2}=\left(1+\frac{a_{1} U^{* 2} \delta}{2 m^{*}}+U^{* 2} \delta^{2}\right)^{-1}$ has been introduced for convenience. The first point to note is that the dynamics of the cantilevered system tends to that of pure TG (see Barrero-Gil et al. 2010) for long beams $(\delta \ll 1)$, since the correction factors $k_{1}$ and $k_{2}$ tend to 1 . Second, the reduced velocity at which Galloping starts $U_{g}^{*}$ is given by the point at which $A^{*}=0$, that is

$$
U_{g}^{*}=\frac{4 m^{*}}{a_{1}}\left(\zeta+\zeta_{E}\right)=\frac{4 m^{*}}{a_{1}}\left(\zeta+\frac{\Theta^{2} R_{L}}{2 m \omega_{N}\left(1+C_{p}^{2} R_{L}^{2} \omega_{N}^{2} k_{1}\right)}\right),
$$

which indicates that Galloping occurs at lower velocities as $\delta$ increases since $k_{1}$ increases too. Third, $\delta$ increases the frequency of oscillations $\left(k_{1}>1\right)$ and diminishes the amplitude of oscillations $\left(k_{2}<1\right)$, more strongly as $m^{*}$ is lower. Finally, for large reduced velocities $A^{*}$ tends to an asymptotic finite value, whereas in pure TG the amplitude of oscillations grows with the reduced velocity without limit. The asymptotic value is given by

$$
A_{\infty}^{*}=\left(-\frac{8 m^{*} a_{1}}{3 a_{3} \delta\left(a_{1}+2 m^{*} \delta\right)}\right)^{1 / 2}
$$

which, as expected, tends to infinite when $\delta$ tends to zero (large beams). Note also that $A_{\infty}^{*}$ does not depend on the electrical properties (piezoelectric sheets properties and electrical resistance).

For very large $m^{*}$ (say, greater than 100) and $a_{1} U^{* 2} \delta / m^{*} \ll 1$, which are usual conditions when the fluid medium is air, $k_{2} \approx\left(1+U^{* 2} \delta^{2}\right)^{-1}$ which indicates that unless $U^{*} \delta$ is small, the amplitude of oscillations is significantly reduced with respect to the case of pure TG. For instance, for $U^{*} \delta$ of order unity the amplitude of galloping oscillations in cantilever arrangement is expected to be of the order of half times the amplitude predicted by pure TG model $\left(k_{2} \sim\right.$ $1 / 2)$. This indicates that pure TG modeling is not adequate for those situations in which $U^{*} \delta \sim 1$.

Finally, the asymptotic limit for amplitude of oscillations when $m^{*}$ is very large is $A_{\infty}^{*} \approx$ $\left(-4 a_{1} /\left(3 a_{3} \delta^{2}\right)\right)^{1 / 2}$. A word of caution is needed here. A main assumption was made during the analysis with respect to the angle of attack, since it was assumed that $(y) / U \sim A^{*} / U^{*}<\alpha_{L I M}$ and $3 y /(2 L) \sim \partial A *<\alpha_{L I M}$ meaning $\alpha_{L I M}$ an angle at which the approximation $\tan \left(\alpha_{L I M}\right) \approx$ $\alpha_{L I M}$ is still reasonable. In order to fulfill the assumption made, these conditions should be checked. Obviously for situations where this assumption is not valid, either a higher order of approximation or a numerical solution of the system given by Eqs. 4.2, 4.3, 4.5, 4.11, and 4.12 
is needed. In addition, it should be noted that the elastic model of the cantilever beam is give only by an equivalent bending stiffness and neither torsional nor buckling effects are considered. When $\delta$ is very small, elastic response of the cantilever is expected to be more complex with parasitic torsion motions and a more refined elastic model could be needed.

\subsubsection{Electrical power}

At the steady-state of harmonic oscillations, from Eq. 4.22 one may get

$$
P_{E}=m \omega_{N}^{3} D^{2} \zeta_{E} A^{* 2} \omega^{* 2},
$$

and therefore from Ecs. 4.26a and 4.27

$$
P_{E}=m \omega_{N}^{3} D^{2} \zeta_{E} \frac{4 U^{*}}{3 a_{3}}\left(4 m^{*}\left(\zeta+\zeta_{E}\right)-a_{1} U^{*}\right) k_{1} k_{2}
$$

This expression gives the electrical power as a function of the geometrical $\left(a_{1}, a_{3}\right)$ and mechanical $\left(m^{*}, \zeta\right)$ properties of the prism, piezoelectric sheet properties $\left(\zeta_{E}\right)$, flow speed $\left(U^{*}\right)$, and length aspect of the beam $(\delta)$. Not that it may be useful for designing purposes in order to optimize the electrical power output by choosing appropriately the mechanical properties and the electrical resistance.

The efficiency can be now obtained from Eqs. 4.25 and 4.30,

$$
\eta=\frac{8 m^{*} \zeta_{E}}{3 a_{3} U^{* 2}}\left(4 m^{*}\left(\zeta+\zeta_{E}\right)-a_{1} U^{*}\right) k_{1} k_{2}
$$

It is interesting to note that

$$
k_{1} k_{2}=\frac{1+\frac{1_{1} U^{* 2} \delta}{2 m^{*}}}{1+\frac{a_{1} U^{* 2} \delta}{2 m^{*}}+U^{* 2} \delta^{2}}=\left(1+\frac{U^{* 2} \delta^{2}}{1+\frac{a_{1} U^{* 2} \delta}{2 m^{*}}}\right)^{-1}
$$

recovers the effect due to the cantilever, and takes a value lower than 1 for $\delta>0$, meaning that there is a drop in the electrical power associated to the cantilever arrangement. For aero-elastic situations in which $m^{*} \gg 1, k_{1} k_{2}$ tends to $\left(1+\delta^{2} U^{* 2}\right)^{-1}$.

From the practical side, it may be of interest to know the maximum achievable efficiency $\eta_{\max }$ for a given configuration as well as the reduced velocity (the wind speed) at which the efficiency is maximum $U_{\eta_{\max }}^{*}$. This can be done by setting $\partial \eta / \partial U^{*}=0$ from Eq. 4.32, and solving. If $m^{*} \gg 1$ (aeroelastic situation) one arrives to the following equation

$$
U^{*}-2 U_{g}^{*}-\delta^{2} U^{* 2}\left(4 U_{g}^{*}-3 U^{*}\right)=0,
$$

which should be solved to find $U_{\eta_{\max }}^{*} ; U_{g}^{*}=4 m^{*}\left(\zeta+\zeta_{E}\right) / a_{1}$ is the reduced velocity at which 
Galloping starts. An amenable explicit expression for $U_{\eta \max }^{*}$ has not been found but a simple analysis of Eq. 4.34 shows that in the limit $\delta \rightarrow 0$, then $U_{\eta_{\max }^{*}}$ tends to $2 U_{g}^{*}$. On the contrary, when $\delta \rightarrow \infty$ then $U_{\eta_{\max }}^{*}$ tends to $4 U_{g}^{*} / 3$. For other conditions of the cantilever beam's length $4 U_{g}^{*} / 3<U_{\eta_{\max }}^{*}<2 U_{g}^{*}$. It is proven analytically that $U_{\eta_{\max }}^{*} / U_{g}^{*}$ depends on the product $U_{g}^{*} \delta$. Fig. 4.2 shows $U_{\eta_{\max }}^{*} / U_{g}^{*}$ variation with $U_{g}^{*} \delta$ which has been obtained numerically solving Eq. 4.34. It has been found that a good fitting law is

$$
\frac{U_{\eta_{\max }^{*}}^{*}}{U_{g}^{*}}=c \approx \frac{2}{3}\left(2+e^{\left(-\delta U_{g}^{*}\right)}\right),
$$

where $c$ is a variable introduced for convenience. Substituting this law into Eq. 4.32, taking $U^{*}=U_{\eta_{\max }}^{*}=c U_{g}^{*}$, it is found that

$$
\eta_{\text {max }}=-\frac{a_{1}^{2}}{6 a_{3}} \frac{1}{1+\zeta / \zeta_{E}}\left(\left(\frac{4(c-1)}{c^{2}}\right) \frac{1}{1+\frac{16 c^{2} \delta^{2} m^{* 2}\left(\zeta+\zeta_{E}\right)^{2}}{a_{1}^{2}}}\right)=\eta_{\text {aero }} \eta_{\text {piezo }} \eta_{\text {cant }}
$$

where the value of $U_{g}^{*}=4 m^{*}\left(\zeta+\zeta_{E}\right) / a_{1}$ has been taken into account. This indicates that the maximum achievable efficiency depends on the geometry of the cross-section of the prism by means of an aerodynamic efficiency factor, namely $\eta_{\text {aero }}=-a_{1}^{2} /\left(6 a_{3}\right)$, on the ratio of mechanical damping to piezoelectric damping in a second efficiency factor $\eta_{\text {piezo }}=\left(1+\zeta / \zeta_{E}\right)^{-1}$ and, finally, on the cantilever effect which is defined by another efficiency factor which recovers the cantilever influence,

$$
\eta_{\text {cant }}=\left(\frac{4(c-1)}{c^{2}}\right) \frac{1}{1+\frac{16 c^{2} \delta^{2} m^{* 2}\left(\zeta+\zeta_{E}\right)^{2}}{a_{1}^{2}}}
$$




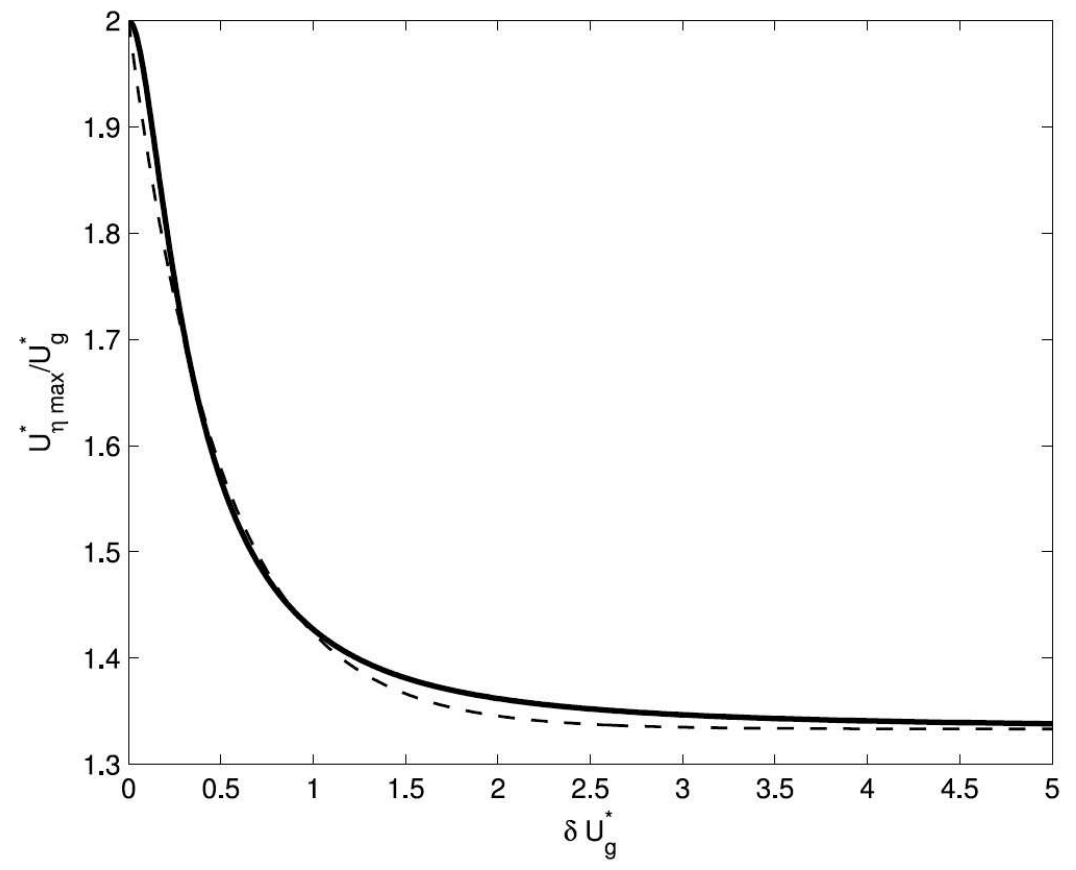

Figure 4.2: $U_{\eta \max }^{*} / U_{g}^{*}$ dependence with $\delta U_{g}^{*}$. Solid line stands for numeric solution of Eq. 4.34 whereas dashed line shows the approximation given in Eq. 4.35.

Observe that $\eta_{\text {cant }} \leq 1$ for $\delta>0$ since $3 / 4 \leq 4(c-1) / c^{2} \leq 1$ in the range of interest $(4 / 3 \leq c \leq 2)$. In other words, there is a drop in $\eta_{\max }$ in the cantilever arrangement due to the induced rotation angle. Discussing qualitatively the role of the different parameters in $\eta_{\max }$, it follows from Eq. 4.36 that it is beneficial to have high values of $a_{1}$, low values of $-a_{3}$, low $\zeta$, and low $\delta$. In addition, it can be seen that there must exist an optimal value of $\zeta_{E}$ since $\partial \eta_{\text {piezo }} / \partial \zeta_{E}>0$ and $\partial \eta_{\text {cant }} / \partial \zeta_{E}<0$.

\subsection{Model validation}

A detailed experimental campaign of piezoelectric energy harvesting from aero-elastic Galloping was carried out by Zhao et al. (2013). The experimental arrangement consisted in a rigid squaresection cylinder fixed to the free end of a cantilevered beam of length. For electricity conversion piezoelectric sheets were attached to the base of the beam. Table 4.1 lists the mechanical and piezoelectric parameters in their experiments.

In Fig. 4.3a, a comparison between experimental results and analytical results (Eq. 4.31) for the mean electrical power dissipated at the electrical resistance as a function of the wind speed is presented. Good agreement can be noted. It is also shown, for illustrative purposes, the analytical prediction obtained without considering the cantilever effect (that is, taking $\delta=$ $0)$. It is noteworthy that the mean electrical power is considerably less for the cantilevered arrangement (around $52 \%, 46 \%$ and $42 \%$ less for $\mathrm{U}=4,5$, and $6 \mathrm{~m} / \mathrm{s}$ respectively). 
Table 4.1: Physical properties in Zhao et al. (2013) experiments. Source: Values of aerodynamic coefficients $a_{1}$ and $a_{3}$ are taken from Novak and Tanaka 1974.

\begin{tabular}{lll}
\hline Parameter & Configuration 1 & Configuration 2 \\
\hline$m(\mathrm{Kg})$ & 0.029 & 0.029 \\
$K(\mathrm{~N} / \mathrm{m})$ & 381.6 & 381.6 \\
$C_{p}(\mathrm{nF})$ & 180 & 180 \\
$\Theta(\mathrm{N} / \mathrm{V})$ & $3.7310^{-4}$ & $3.7310^{-4}$ \\
$R_{L}(\mathrm{k} \Omega)$ & 105 & 105 \\
$L_{b}(\mathrm{~m})$ & 0.15 & 0.15 \\
$D(\mathrm{~m})$ & 0.04 & 0.04 \\
$L(\mathrm{~m})$ & 0.15 & 0.10 \\
$\rho\left(\mathrm{Kg} / \mathrm{m}^{3}\right)$ & 1.2 & 1.2 \\
$U(\mathrm{~m} / \mathrm{s})$ & $2-8$ & $2-8$ \\
$m^{*}$ & 107.6 & 161.5 \\
$\zeta$ & 0.005 & 0.004 \\
$\omega_{N}(\mathrm{rad} / \mathrm{s})$ & 53.7 & 53.7 \\
$\delta$ & 0.4 & 0.4 \\
$a_{1}$ & 2.3 & 2.3 \\
$a_{3}$ & -18 & -18 \\
\hline
\end{tabular}

Fig. 4.3b shows the efficiency given by the theoretical model (Eq. 4.32) along with experimental results. Again, the analytical prediction obtained without considering the cantilever effect is shown for completeness. As it can be seen, there is a drop in the efficiency for the cantilever arrangement with respect to the case of pure TG. In addition, the velocity at which the efficiency is maximum $U_{\eta_{\text {max }}}^{*}$ is lower than that of pure TG. It can also be noted that predictions given by Eqs. $4.35\left(U_{\eta_{\max }^{*}}^{*} \approx 2.4\right)$ and4.36 $\left(\eta_{\max } \approx 0.009\right)$ are in reasonable agreement with experimental results. It is interesting to note that in this case $\eta_{\text {aero }}=0.049, \eta_{\text {piezo }}=0.36$ and $\eta_{\text {cant }}=0.48$ which shows that efficiency losses by cantilever arrangement are significant. Efficiency losses due to cantilever effect are significant because the presence of the cantilever adds an additional angle $\theta$ to the angle of attack, which results in lower achievable galloping amplitudes and less energy transfer. In addition, as already anticipated the cross-section geometry of the prism dictates the maximum achievable aeroelastic efficiency. Galloping response of a square prism is weak in comparison with other cross-section geometries like triangular, or D-type (Barrero-Gil et al. 2010, Bokaian and Geoola 1983).

Finally, Fig. 4.4a and 4.4b show experimental results from Zhao et al. (2013) and analytical predictions for a configuration with a larger value of the mass ratio. Predictions for $U_{\eta_{\text {max }}}^{*}$ is 2.85 (Eq. 4.34) and for $\eta_{\max } 0.005$ (Eq. 4.36), which are in reasonable agreement with experimental results. It is worth noting that even at a relatively large value of $\delta$, where the hypothesis of low rotating angle and low angle of attack considered in the theoretical model may be more doubtful, the degree of agreement is fairly good. As predicted by Eq. 4.28, the wind speed at which Galloping starts has been increased with respect to the case of lower $m^{*}$, and the harvested power has decreased (as can be deduced from Eq. 4.31).

To see more clearly the precise effect of $\delta$, it is necessary to consider values of configuration and make $\delta$ a free parameter. Fig. 4.5 shows the amplitude of oscillations and electrical 


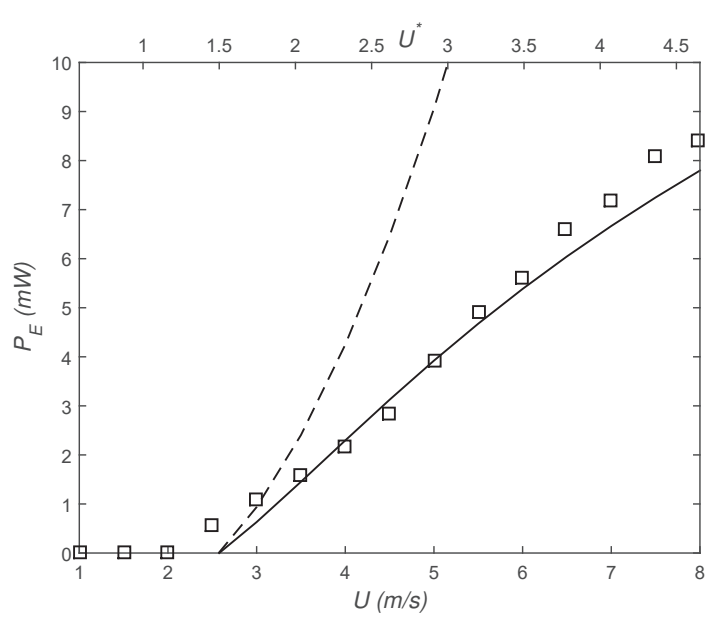

(a)

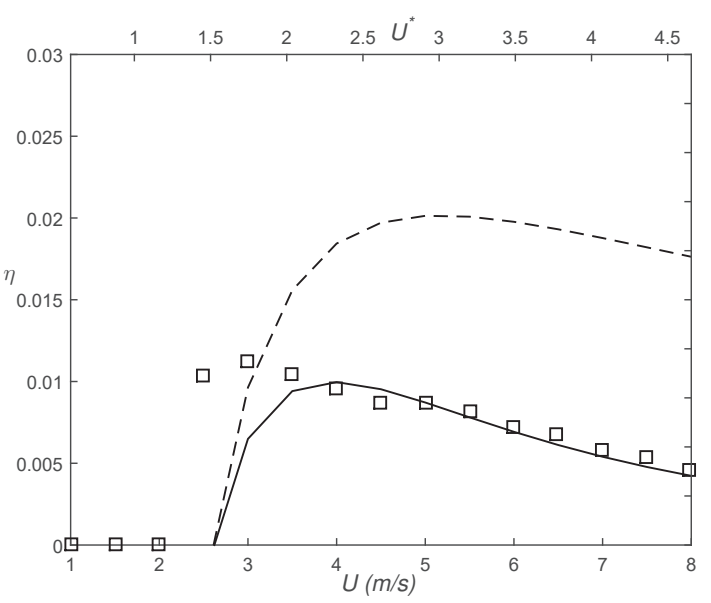

(b)

Figure 4.3: (a) Mean power variation with airspeed. (b) Efficiency dependence with airspeed. Open square stands for experimental results from Zhao et al. (2013) in the configuration where $m^{*}=101.7$ and $\delta=0.4$. Solid line stands for analytical predictions from the model (Eqs. 4.31 and 4.32). Dashed line stands for analytical solution without considering the cantilever aerodynamic effect (pure TG).

power as a function of $\delta$ and fluid velocity. As can be seen, for values of flow velocity close to that of starting Galloping, $\delta$ barely affects the response; however, when flow velocity gets higher, amplitude growth with the reduced velocity $U^{*}$ is diminished with $\delta$, even getting into an asymptotic value as was anticipated in Eq. 4.29. The behavior of electrical power is qualitatively the same and it can be seen that, for a fixed wind speed, pure TG seems to be always a more convenient option than the cantilever one. This is because the cantilever always introduces an efficiency factor (see Eq. 4.37) to the energy conversion system. Nevertheless, taking into account engineering design aspects, it seems clear that cantilever configurations can be easily implemented, with low mechanical parasitic damping and low costs, whereas pure TG requires a more sophisticated mounting system to maintain the attitude of the prism.

\subsection{Conclusions}

A theoretical model representing the dynamics of oscillatory motion of a piezo-galloping cantilever system coupled to a purely resistive energy harvesting circuit has been considered. A nonlinear Quasi-steady approximation of the aerodynamic force has been utilized and the influence of the rotation angle due to the cantilever arrangement considered. An analytical solution has been given which has been compared favorably with experimental results from Zhao et al. (2013). This constitutes a theoretical guide to optimize the power by selecting appropriately the mechanical properties and tuning the electrical resistance. In addition, the model has been analyzed in some detail with the following findings: 


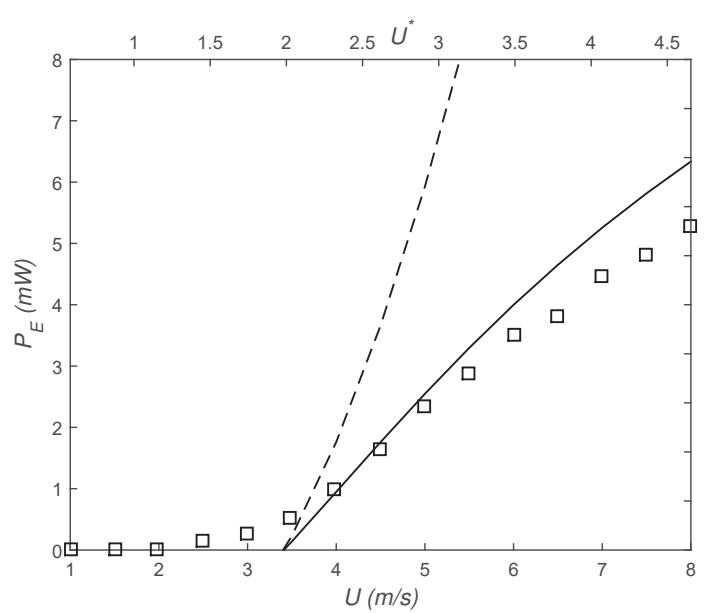

(a)

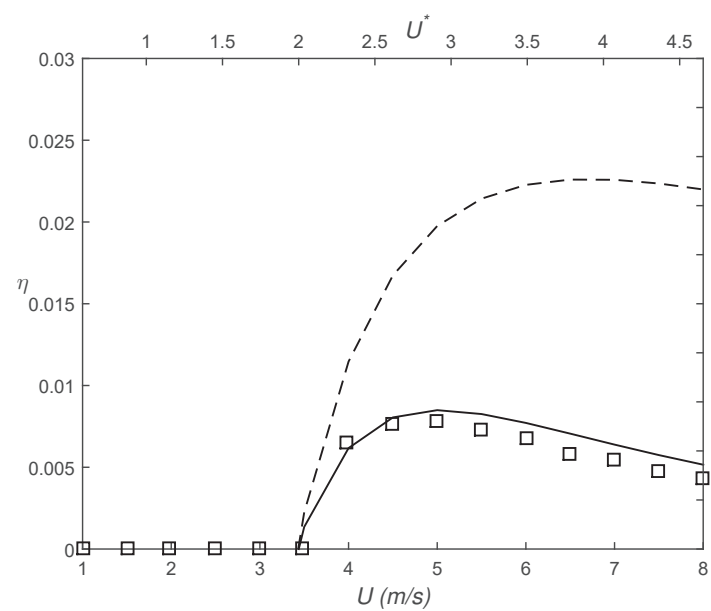

(b)

Figure 4.4: (a) Mean power variation with airspeed. (b) Efficiency dependence with airspeed. Open square stands for experimental results from Zhao et al. (2013) in the configuration where $m^{*}=152.6$ and $\delta=0.4$. Solid line stands for analytical predictions from the model (Eqs. 4.31 and 4.32). Dashed line stands for analytical solution without considering the cantilever aerodynamic effect (pure TG).

- The electrical power output depends significantly on the length aspect of the cantilever $(\delta)$. The lower the length aspect of the beam the lower the level of energy harvesting. For very high values of mass ratio (typical for aeroelastic situations) electrical power output is proportional to $\left(1+\delta^{2} U^{* 2}\right)^{-1}$ (see Eq. 4.33).

- The reduced velocity at which galloping oscillations starts (and electrical power output) depends slightly on $\delta$ and diminishes with respect to the value predicted by pure TG modeling.

- The electrical power output tends to an asymptotic finite value for a large reduced velocity $U^{*}$, whereas in pure TG the electrical power output grows without limit with $U^{*}$. This asymptotic value is due to the cantilever effect on galloping oscillation amplitude.

- The reduced velocity at which the efficiency is maximum $\left(U_{\max }^{*}\right)$ depends on $\delta$. For very large beams $(\delta \rightarrow 0)$ it is found that $U_{\text {max }}^{*} \rightarrow 2 U_{g}^{*}$, whereas for short beams $U_{\text {max }}^{*}$ approaches to $4 U_{g}^{*} / 3$. Observe that in a real site with a certain environmental wind speed $U_{g}^{*}$ can be adjusted (by changing $m^{*}$ for example, see Eq. 4.28) in order to work in the point of maximum efficiency.

- Maximum attainable efficiency diminishes with the length aspect ratio of the cantilever (see Eq. 4.37). Efficiency losses by cantilever arrangement may be significant.

- With regards to the dynamics of the prism, the normalized amplitude of oscillations $A^{*}$ for cantilever Galloping is lower than that of pure Transverse Galloping. Conversely, the normalized frequency of oscillations is larger. $A^{*}$ tends to an asymptotic finite value for a large reduced velocity $U^{*}$. 


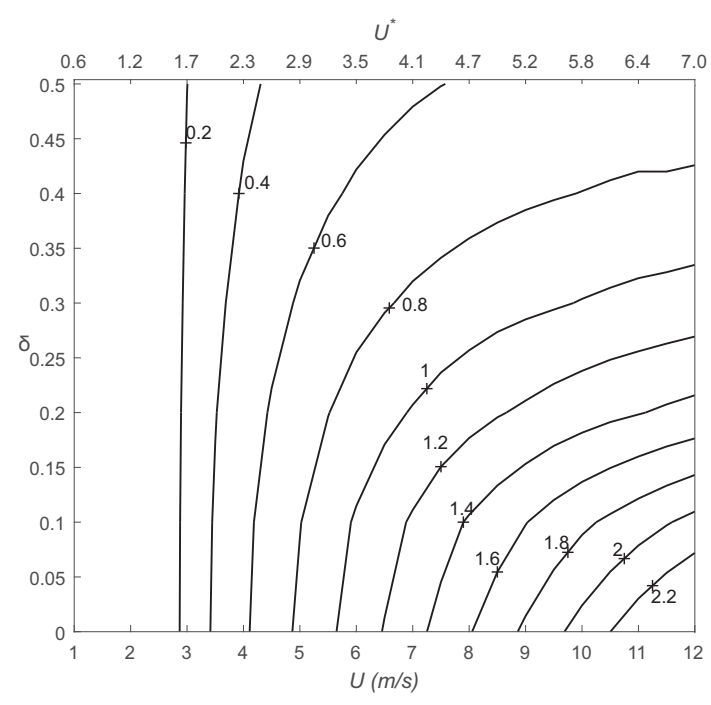

(a)

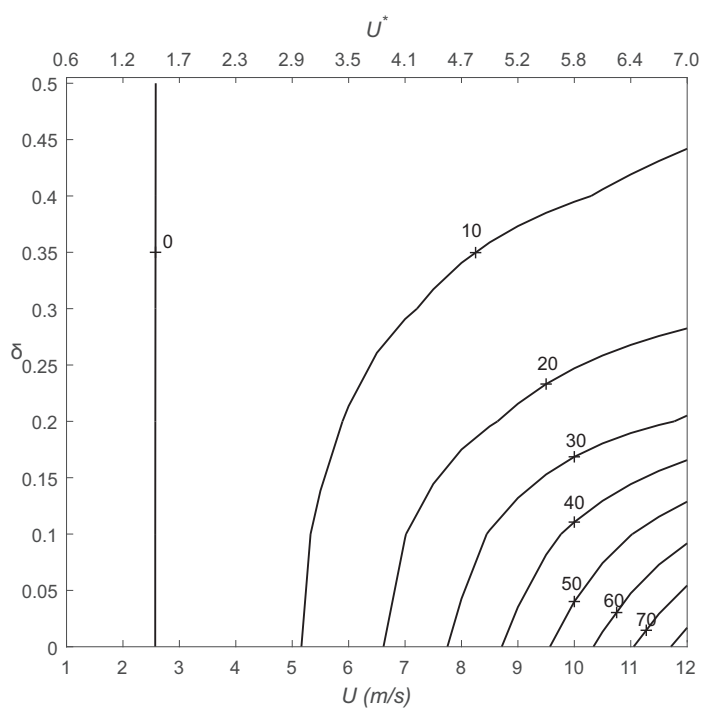

(b)

Figure 4.5: (a) Contour plot of $A^{*}$ dependence with $U^{*}$ and $\delta$. Governing parameters correspond to those of Configuration 1. (b) Contour plot of $P_{E}$ dependence with $U^{*}$ and $\delta$. Governing parameters correspond to those of Configuration 1.

Some open questions derived from the present study require further insight, like the effect due to torsional bending of the cantilever, piezoelectric sheet size and placement, or the optimal cross-section geometry sections. In addition, in the present analysis the electrical load at which power is dissipated has been considered constant. It could be of interest to see if there exists an optimal electrical load for each flow speed in order to improve the power extracted. 



\section{Chapter 5}

\section{Dual mass system for enhancing energy extraction}

\subsection{Introduction}

Vortex Induced Vibrations (VIV) occur in many engineering structures, like slender chimney stacks, tall buildings, electric power lines, long-span bridges, flexible structures in petroleum production, or in the tubes of heat exchange devices. Usually, VIV are considered an unwanted effect since they may reduce seriously the lifetime of the structure by fatigue loading, compromise its performance or, even worse, its integrity. However, VIV can also be used to extract energy from the surrounding flow, as was pointed out by Bernitsas and Raghavan (2008) who proposed the application of VIV to convert hydrokinetic energy from ocean/river currents to electricity. Additional studies in the field have been reported by Sanchez-Sanz et al. (2009), Barrero-Gil et al. (2012), Nishi (2014), Mackowski and Williamson (2013) and Meliza et al. (2011). The basic idea is to convert part of the kinetic energy of the incoming fluid flow into oscillatory mechanical energy via VIV. In this way, the kinetic energy of the oscillations can be used for practical purposes like, for instance, electricity production using either an electromagnetic, piezoelectric, or electrostatic conversion system.

VIV have their origin on the large-scale vortices that are shed from a bluff-body under the action of an incoming flow when the Reynolds number is low to moderately high (say, in the range from $10^{2}$ to $10^{4}$ ). These vortices are shed periodically to the body wake at a frequency that is related to the incoming flow speed and body cross-section dimension via the Strouhal number. This periodic shedding of vortices creates a fluctuating lift force on the body and this causes the body to oscillate over a range of flow velocities whenever vortex shedding frequency is close to the natural frequency of the structure. More specifically, when part of the lift force is in phase with body oscillations the fluid exerts work on the body and energy is transferred from the fluid into the oscillating system. The interaction between the incoming flow, vortex shedding, fluctuating force on the body and its response is a very complex problem far from being completely understood. For a detailed introduction to the subject, the reader is referred to several review articles: Bearman (1984), Parkinson (1989), Williamson and Govardham (2004), or Sarpkaya (2004).

The use of a dual mass configuration to enhance energy extraction from vibration sources of a generic nature (not specifically related to fluid-structure interaction phenomena) was proposed by Tang and Zuo (2011). In their work, they considered the case of a linear vibrating system where one of the masses is excited by an external oscillating force. Two years later, Nishi (2013) proposed enhancing power extraction from VIV by means of using a tuned dual mass configuration. To study the dynamics of the system he proposed a two-degrees-of-freedom model where VIV fluid forces on the main mass (a circular cylinder exposed to the flow) were described 
by a wake oscillator model. Within the hypothesis of his model, he found enhancement of power extraction as compared to the single mass configuration whenever an appropriate combination of the dual mass parameters was chosen.

In this Chapter of the Thesis, energy extraction from a VIV dual mass system is studied and discussed. The study focuses on the relation between the mechanical parameters of the dual mass system and the efficiency of energy conversion. More specifically, two main questions are asked and answered: a) what is the maximum efficiency attainable in a dual-mass system?, and b) can a dual-mass system increase the range of flow velocities where energy extraction is efficient?. To this end, a simplified two-degree-of-freedom model has been developed, where fluid forces are taken from a experimental database (Hover et al. 1998), and this is a relevant departure from the previously referenced work of Nishi (2013) in which he used a wake oscillator model. Also, the present work can be regarded as a further extension of the single-mass oriented previous work of one of the authors, Barrero-Gil et al. (2012). The two-degree-of freedom model can provide a useful general view of the system response when varying mechanical and operational parameters. The main aim is to reach a compromise between the simplicity and visibility of an analytical approach and the completeness of a numerical or experimental whole domain characterization. The validity of the fluid force model used in this research is backed by Sarpkaya (2004). In this review it is remarked that force-transfer coefficients obtained by forced vibrations are reliable for designing proposes and the experiments between two VIV in different Reynolds numbers (from laminar to Re larger than 15000) are sufficiently alike to extract reliable physical information in an idealized way.

In Section 5.2, a mathematical model of VIV with a dual mass system is introduced and the method to obtain solutions from it is discussed. A comparison between single mass configurations and dual mass systems is made in Section 5.3, where it is shown that dual mass systems can be advantageous for energy harvesting purposes whenever appropriately designed. Illustration examples are given in Section 5.4 for suitable and unsuitable dual mass reconfiguration aims and, finally, Section 5.5 presents some concluding remarks.

\subsection{Mathematical model for VIV with dual mass system}

\subsubsection{Mathematical model}

A simplified mathematical model paradigm is considered for more complex systems, which consists of a spring-mounted circular cylinder of mass $m_{1}$ under the action of a fluid flow and a secondary elastically supported mass $m_{2}$ (DM) which is not exposed to the aforementioned flow. Both masses are restricted to move only along the normal direction to the flow, which is the $\mathrm{y}$-axis in the defined coordinate system if the flow is parallel to x-axis (see Fig. 5.1). The circular cylinder is linked to a platform which acts as ground by a linear spring $k_{1}$, and to the DM with another linear spring $k_{2}$. The circular cylinder can experience vortex shedding fluid forces and develop VIV. Oscillations of the circular cylinder excite the motion of the DM. To account for dissipative effects a linear viscous damper $c_{1}$ is considered in the clamp of the circular cylinder to the ground. It is possible to consider a second dissipation term between 
the circular cylinder and the dual mass (along with $k_{2}$ ). However, for sake of simplicity it can be seen that this additional damping term does not provide more information to the afterward mathematical analysis for obtaining efficiency optimization, as dissipation terms have always negative impact on the energy transference. That is why it only included one dissipation term in order to take into account a source of dissipation effect and remain in balance with simplicity of mathematical formulation. Energy harvesting takes place in an electrical generator placed between the DM and the fixed platform. This electric generator is modeled, for the sake of simplicity, as a mechanical linear viscous damper $c_{E}$, which can be considered as a simplified model of an electromagnetic generator. The electrical generator harvests the power through a reaction force to the mechanical system. This force is characterized by a linear velocity dependent term $\left(c_{E} \dot{(}(y)\right)$. The magnitude of $c_{E}$ depends on the characteristics of the electromagnetic generator, namely the flux density of the permanent magnets, the reclutance of the iron core of the armature, the number os turns of the armature winding, and the electrical load. For more information about the generator architecture and electromechanical coupling, the reader is referenced to El Hami et al. (2001), Vicente-Ludlam et al. (2014).

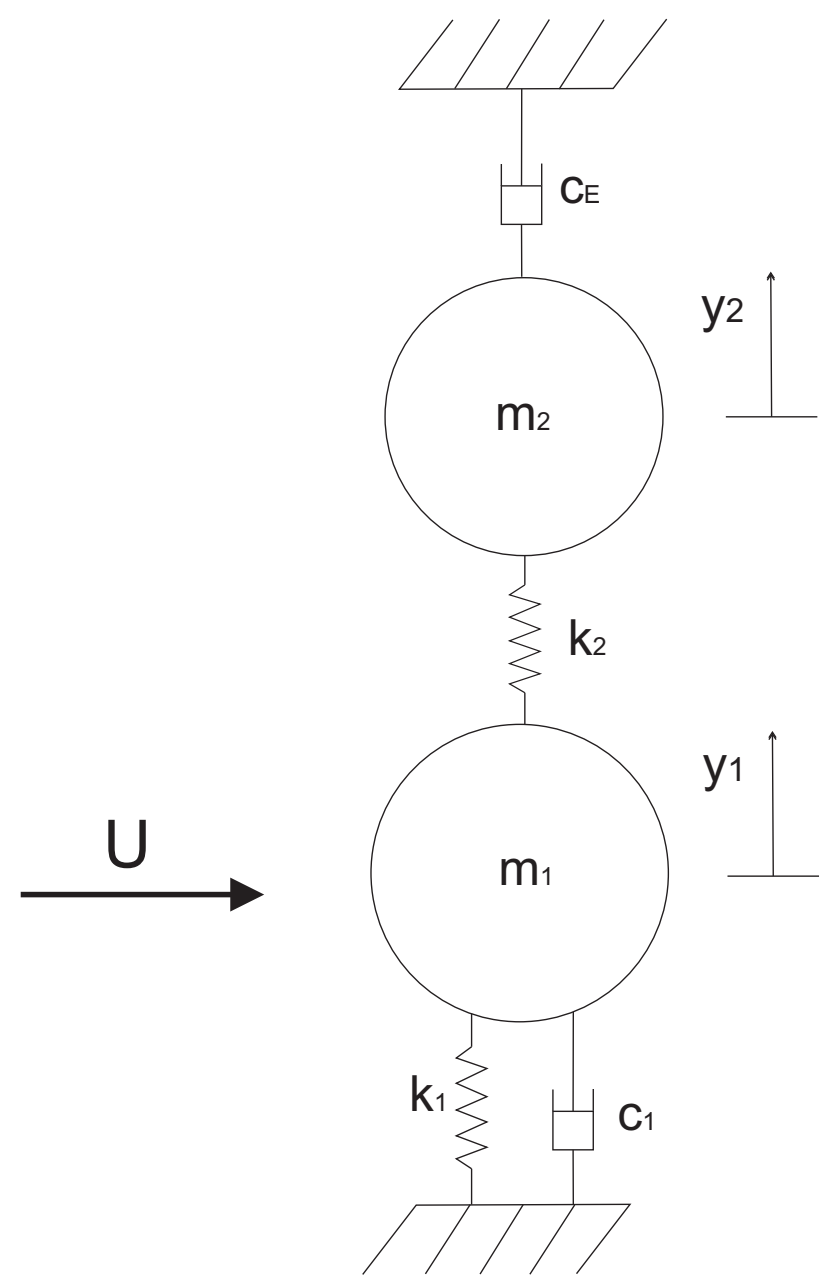

Figure 5.1: Sketch of the VIV of a circular cylinder in a dual mass configuration. The undisturbed direction of the inflow velocity is indicated perpendicular to the displacement of the masses. 
Balance between inertia, damping, stiffness, and fluid forces in the system yields the following system of two ordinary differential equations for the circular cylinder displacement $y_{1}$ and DM displacement $y_{2}$,

$$
\begin{gathered}
m_{1} y_{1}^{\prime \prime}+k_{1} y_{1}-k_{2}\left(y_{2}-y_{1}\right)+c_{1} y_{1}^{\prime}=F_{f}, \\
m_{2} y_{2}^{\prime \prime}+k_{2}\left(y_{2}-y_{1}\right)+c_{E} y_{2}^{\prime}=0,
\end{gathered}
$$

where $F$ is the cross-flow fluid force acting on the circular cylinder.

The complex nature of the flow pattern around the circular cylinder undergoing VIV makes an analytical-empirical formulation of $F$ very difficult. Ideally, one would like to obtain $F$ by solving the equations of structural motion coupled to the fully nonlinear equations of fluid flow but, unfortunately, this approach is usually computationally too expensive. Therefore, phenomenological approaches are usually followed, as those based on wake-oscillator models (Fachinetti et al. 2004, Nishi, 2009 and 2013), where the equation of structural motion is coupled with a nonlinear oscillator equation that describes the cross-fluid force. There are several wake oscillator models but comparisons with free vibration experiments have not been fully satisfactory, since none of them can describe simultaneously all relevant aspects of VIV, like the amplitude and frequency of oscillations dependence with the fluid velocity, or the range of flow velocities where synchronization takes place. Another approach, closer to the actual VIV phenomenon, consists on the use of fluid force coefficients measured in controlled forced vibration experiments (see Sarpkaya, 1978, Gabbai and Banaroya 2005). Sarpkaya (2004), stated that the force coefficients from forced vibrations are suitable for a wide range of Reynolds numbers design purposes (Re from laminar to more than 15000). Under forced vibrations of the circular cylinder, experiments show that in the lock-in region (or resonance range), where oscillations are nearly sinusoidal with fixed amplitude and frequency of oscillations, the fluid force on the circular cylinder can be described to a good approximation as

$$
F_{f}=\frac{1}{2} \rho U^{2} D L\left(-c_{L a} \sin (\omega t)+c_{L v} \cos (\omega t)\right),
$$

where $\rho$ is the fluid density, $U$ the flow velocity, $D$ and $L$ the cylinder diameter and submerged length, $c_{L v}$ is the velocity-coupling fluid force coefficient, and $c_{L a}$ is the inertia-coupling fluid force coefficient, and $\omega$ the cylinder oscillation frequency, which is initially unknown and must be obtained as part of the solution. $c_{L a}$ and $c_{L v}$ depend mainly on the cylinder oscillation characteristics, namely the dimensionless amplitude of oscillations $A^{*}=A / D$ and the true reduced velocity $V^{*}=U /(\omega D)$, which is the inflow velocity normalized with the characteristic length scale $D$ and a characteristic timescale $\omega^{-1}$. This dependence was obtained, for example, by Hover et al. (1998) by forced oscillation experiments in a water channel. In Hover's paper, a set of forced vibration experiments are presented. The Reynolds number is maintained fixed in the whole course of the measurements $(R e=3800)$. Nevertheless, as has been stated in the Introduction Section, the forced experiments of a VIV cylinder are suitable for designing purposes in a large range of Reynolds number. The experiments are carried out in the MIT's towing tank.

Introducing a dimensionless time $\tau=\omega_{1} t$, where $\omega_{1}$ is natural frequency of oscillations for 
the circular cylinder when DM is not present, $\omega_{1}=\left(\frac{k_{1}}{/} m_{1}\right)^{\frac{1}{2}}$, a reduced mass $m^{*}=\frac{m_{1}}{/}\left(\rho D^{2} L\right)$, a mass ratio between masses $\mu=\frac{m_{2}}{l} m_{1}$, dimensionless displacements for the circular cylinder $\eta_{1}=\frac{y_{1}}{D}$ and for the DM $\eta_{2}=\frac{y_{2}}{D}$, stiffness ratio $\Omega=\left(k_{2} / k_{1}\right)^{\frac{1}{2}}$, damping coefficients $\zeta_{1}=\frac{c_{1}}{2 \omega_{1} m_{1}}$ and $\zeta_{E}=\frac{c_{E}}{2 \omega_{1} m_{1}}$, and a reduced velocity $U^{*}=\frac{U}{\omega_{1} D}$, which is the dimensionless velocity associated with the natural frequency. It should be stressed the difference between reduced velocity $U^{*}$ and true reduced velocity defined before as $V^{*}$, which is $V^{*}=U^{*} \omega^{*}$, being $\omega^{*}=\omega / \omega_{1}$. The first one is linear with the physical magnitude $U$, whereas the last one $V^{*}$ depends both on $U$ and the frequency of oscillation $\omega$, which is unknown a priori since it is part of the solution of the fluid-structure interaction problem. Then, Eqs. 5.1 and eq:dimensional2 become:

$$
\begin{gathered}
\ddot{\eta}_{1}+\eta_{1}-\Omega^{2}\left(\eta_{2}-\eta_{1}\right)+2 \zeta_{1} \dot{\eta}_{1}=\frac{1}{2} \frac{U^{* 2}}{m^{*}}\left(-c_{L a} \sin \left(\omega^{*} \tau\right)+c_{L v} \cos \left(\omega^{*} \tau\right)\right), \\
\mu \ddot{\eta}_{2}+\Omega^{2}\left(\eta_{2}-\eta_{1}\right)+2 \zeta_{e} \dot{\eta}_{2}=0 .
\end{gathered}
$$

where $\omega^{*}=\omega / \omega_{1}$, and Eq. 5.3 was taken into account. Based on experimental evidences the steady-state response on the circular cylinder to VIV is close to harmonic oscillations, so that for a good approximation it is possible to consider $\eta_{1}=A^{*} \sin \left(\omega^{*} \tau\right)$ and $\eta_{2}=B^{*} \sin \left(\omega^{*} \tau+\phi\right)$, where $A^{*}=A / D$ and $B^{*}=B / D$ are normalized amplitude of oscillations, and $\phi$ the phase delay between cylinder and dual-mass oscillations. Substituting these expressions into Eqs. and one gets the following algebraic system

$$
\begin{gathered}
A^{*}\left(1-\omega^{* 2}+\Omega^{2}\left(1-\frac{B^{*}}{A^{*}} \cos \phi\right)\right)=-\frac{U^{* 2}}{2 m^{*}} c_{L a}, \\
2 A^{*} \omega^{*}\left(\zeta_{1}-\frac{\Omega^{2}}{2 \omega^{*}} \frac{B^{*}}{A^{*}} \sin \phi\right)=\frac{U^{* 2}}{2 m^{*}} c_{L v}, \\
B^{*}\left(\left(\Omega^{2}-\mu \omega^{* 2}\right) \cos \phi-2 \omega^{*} \zeta_{e} \sin \phi\right)-A^{*} \Omega^{2}=0, \\
\left(\Omega^{2}-\mu \Omega^{* 2}\right) \sin \phi+2 \omega^{*} \zeta_{e} \cos \phi=0,
\end{gathered}
$$

where $c_{L a}=c_{L a}\left(A^{*}, V^{*}\right)$ and $c_{L v}=c_{L v}\left(A^{*}, V^{*}\right)$ as said before.

\subsubsection{Dual-mass VIV response}

There are six dimensionless parameters in the algebraic system defined by Eqs. 5.6a to 5.6d which govern the dynamics. Five of them can be considered as design parameters since they do not depend on the fluid velocity, namely $\zeta_{1}, \zeta_{E}, \Omega, \mu$, and $m^{*}$. The remaining dimensionless parameter, $U^{*}$ accounts for the effect of the fluid velocity. This large space of parameters leads to solutions with different local optimums that makes difficult the interpretation of the results. 

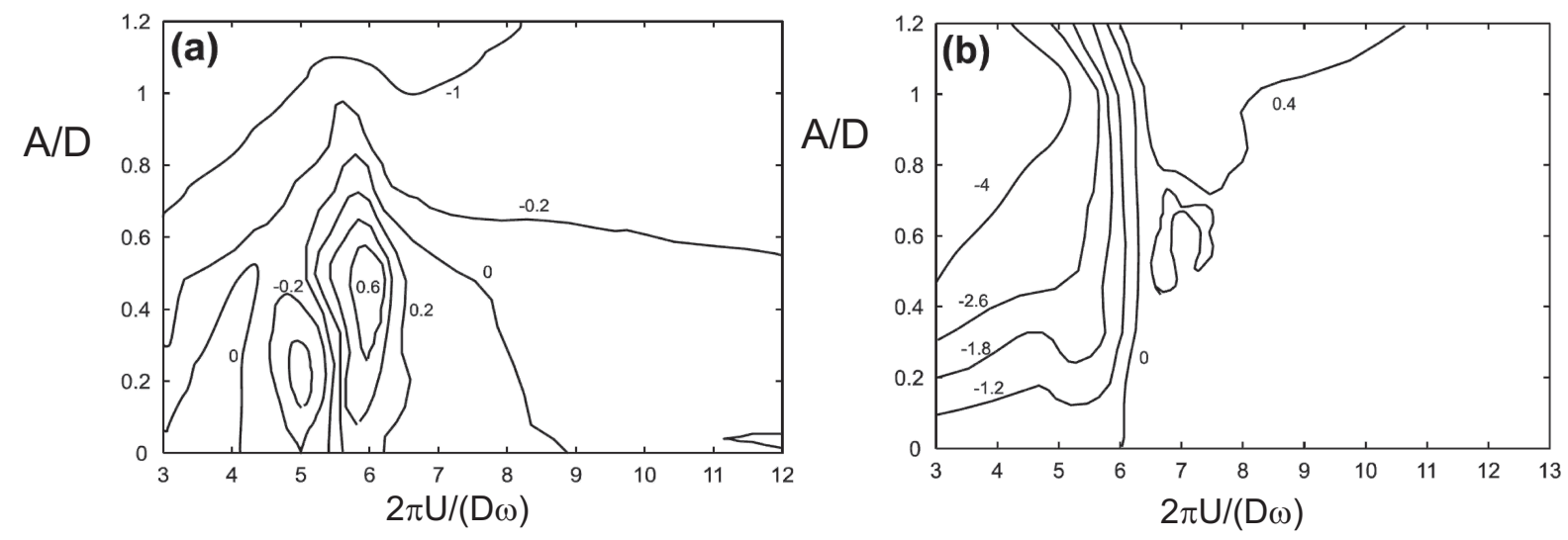

Figure 5.2: Contour plot of $c_{L v}$ (a) and $c_{L a}$ (b) in terms of dimensionless amplitude and the "true reduced velocity" measured by Hover et al. (1998) in experiments from the towing water channel (MIT Test Tank Facility) with constant Reynolds number equal to 3800.

From Eqs. 5.6c and 5.6d, it is possible to obtain the relation between dual-mass and cylinder amplitudes, as well as the phase $\phi$

$$
\begin{aligned}
& K^{*}=\frac{B^{*}}{A^{*}}=\frac{\Omega^{2}}{\sqrt{\left(2 \zeta_{E} \omega^{*}\right)^{2}+\left(\Omega^{2}-\mu \omega^{* 2}\right)^{2}}}, \\
& \cos \phi=\frac{\Omega^{2}-\mu \omega^{* 2}}{\sqrt{\left(2 \zeta_{E} \omega^{*}\right)^{2}+\left(\Omega^{2}-\mu \omega^{* 2}\right)^{2}}}, \\
& \sin \phi=\frac{-2 \zeta_{E} \omega^{*}}{\sqrt{\left(2 \zeta_{E} \omega^{*}\right)^{2}+\left(\Omega^{2}-\mu \omega^{* 2}\right)^{2}}} .
\end{aligned}
$$

As can be seen both $K^{*}$ and $\phi$ depend on the mechanical parameters of the dual-mass system which can be defined at the design stage, namely $\Omega, \mu$, and $\zeta_{E}$, as well as $\omega^{*}$ which is not known a priori.

$\mathrm{S} \omega^{*}$ and $A^{*}$ have to be computed from Eqs. 5.6a and 5.6b. For convenience, an equivalent frequency $\omega_{e q}^{*}$, equivalent mass ratio $m_{e q}^{*}$, and equivalent dimensionless damping $\zeta_{e q}$ are introduced:

$$
\begin{gathered}
\omega_{e q}^{* 2}=\omega^{* 2}-\Omega^{2}\left(1-K^{*} \cos \phi\right), \\
m_{e q}^{*}=\frac{m^{*} \omega_{e q}^{* 2}}{\omega^{* 2}},
\end{gathered}
$$




$$
\zeta_{e q}=\zeta_{E} \frac{\omega^{*}}{\omega_{e q}^{*}}\left(\frac{\zeta_{1}}{\zeta_{E}}+K^{* 2}\right)
$$

After introducing these three new parameters into Eqs. 5.6a and 5.6b a more compact formulation is obtained,

$$
\begin{gathered}
A^{*}\left(1-\omega_{e q}^{* 2}\right)=-\frac{\omega_{e q}^{*} 2 V^{*} 2}{2 m_{e} q^{*}} c_{L a}, \\
2 A^{*} \omega_{e q}^{*} \zeta_{e q}=\frac{\omega_{e q}^{* 2} V^{* 2}}{2 m_{e q}^{*}} c_{L v} .
\end{gathered}
$$

These transformed equations $(5.11 \mathrm{a}, 5.11 \mathrm{~b})$ depend on $m_{e q}^{*}, \zeta_{e q}, V^{*}, c_{L a}$ and $c_{L v}$. By this way, the design space has been reduced from five parameters to two $\left(m_{e q}^{*}\right.$ and $\left.\zeta_{e q}\right)$. Although a pair of $m_{e q}^{*}$ and $\zeta_{e q}$ values can be reached by different combinations of these five, the cylindrical body amplitude results equivalent for each combo. That is, once $c_{L a}$ and $c_{L v}$ dependence with $A^{*}$ and $V^{*}$ is known, the dependence is just on $m_{e q}^{*}, \zeta_{e q}$, and $V^{*}$, i.e. $A^{*}=A^{*}\left(m_{e q}^{*}, \zeta_{e q}, V^{*}\right)$. Eqs. 5.10a, 5.10b, 5.10c, 5.11a and 5.11b allow to compute $A^{*}$ and $\omega_{e q}^{*}$ dependence with $V^{*}$, as well as $A^{*}$ and $\omega^{*}$ dependence with $U^{*}$ in an iterative way if the mechanical configuration is known $\left(\zeta_{1}, \zeta_{E}, \Omega, \mu\right.$ and $\left.m^{*}\right)$. To this end, the following steps have to be given:

- Step 1. A value of the true reduced velocity $V^{*}$ is fixed.

- Step 2. A normalized amplitude of oscillations $A_{i}^{*}$ is prescribed ( $A_{i}^{*}$ ranging from zero to 1.2 in steps of 0.001$)$.

- Step 3. $c_{L a}\left(A_{i}^{*}, V^{*}\right)$ and $c_{L v}\left(A_{i}^{*}, V^{*}\right)$ are computed by $2 \mathrm{D}$ spline interpolation from the experimental database (see Fig. 5.2).

- Step 4. A normalized oscillations frequency $\omega_{j}^{*}$ is prescribed ( $\omega_{j}^{*}$ starts from zero and increases in steps of 0.001).

- Step 5. $K^{*}$ and $\phi$ are computed from Eqs. 5.7 and 6.9, with known mechanical parameters $\Omega, \mu, \zeta_{E}$ and $\omega_{j}^{*}$.

- Step 6. $\omega_{e q}^{*}$ is computed from Eq. 5.10a.

- Step 7. $m_{e q}^{*}$ and $\zeta_{e q}$ are computed from Eqs. 5.10b and 5.10c.

- Step 8. Two residuals, $r e s_{1}=A_{i}^{*}-V^{* 2} \omega_{e q}^{*} c_{L v} /\left(4 m_{e q}^{*} \zeta_{e q}\right)$ and $r e s_{2}=\omega_{e q}^{*}-\left(1+V^{* 2} \omega_{e q}^{* 2} c_{L a} /\left(2 m_{e q}^{*} A_{i}^{*}\right)\right)^{1 / 2}$, are computed.

- Step 9. If both $\left|r e s_{1}\right|<0.01$ and $\left|r e s_{2}\right|<0.01$ the process is finished and solutions $A_{s}^{*}=A_{i}^{*}, \omega_{e q s}^{*}=\omega_{e q}^{*}$, and $\omega_{s}^{*}=\omega_{j}^{*}$ are found.

- Step 10. The true reduced velocity is increased and the procedure is repeated. 
Once $A^{*}, \omega_{e q}^{*}$, and $\omega^{*}$ are available, other related parameters can be computed. For example, the normalized amplitude of oscillations of the DM $B^{*}$ can be obtained from Eq. 5.7, and $\phi$ from Eq. 6.9, so that the full DM response is obtained. Observe that the single-mass response is recovered when $\mu=0$ and $\Omega \rightarrow \infty\left(K^{*}=1, \phi=0, \omega_{e q}^{*}=\omega^{*}, m_{e q}^{*}=m^{*}, \zeta_{e q}=\zeta_{1}+\zeta_{E}\right)$. In this sense, the single-mass configuration can be considered as a particular case of the dual-mass configuration.

\subsubsection{Energy harvesting efficiency}

A conversion factor (or efficiency) $\eta$ can be defined as the time-averaged power $P_{E}$ dissipated at $c_{E}$ divided by the energy flux across the the cylinder section:

$$
\eta=\frac{2 P_{E}}{\rho U^{3} D}
$$

where

$$
P_{E}=\frac{1}{T} \int_{0}^{T} c_{E} y_{2}^{\prime 2} d t=2 m_{1} \omega_{1} \zeta_{E}\left(\omega_{1} D\right)^{2}<\dot{\eta}_{2}^{2}>
$$

$T$ being an averaging time and $<.>$ means averaged value. The efficiency, as described in Eq. 5.12, is presented in the same way as in Barrero-gil et al. (2010), or Groutier et al. (2013), where the power harvested is compared to the available power in the flow in a section equivalent to the diameter of the prism. However, there are other ways of defining such efficiency. For example, the efficiency can be defined as the ratio between harvested power and power available in the flow in a section equivalent to the space swept by the prism during its oscillations. The efficiency defined in Eq. 5.12 stresses the importance of the power extracted while the other one stresses the importance of the power extracted per unit length in the transverse direction. From Eqs. 5.12 and 5.13

$$
\eta_{D M}=\frac{2 m^{*} \zeta_{E} B^{* 2} \omega^{* 2}}{U^{* 3}}=\frac{2 m^{*} \zeta_{E} A^{* 2} K^{* 2}}{V^{* 3} \omega^{*}}
$$

where subscript DM makes reference to dual-mass configuration. Eq. 5.14 can be re-arranged taking into account Ecs. 5.7 to $5.10 \mathrm{c}$ to give

$$
\eta_{D M}=\frac{2 m_{e q}^{*} \zeta_{e q} A^{* 2}}{V^{* 3} \omega_{e q}^{*}} \gamma_{D M}=\chi \gamma_{D M},
$$

with

$$
\chi=\frac{2 m_{e q}^{*} \zeta_{e q} A^{2} *}{V^{* 3} \omega_{e q}^{*}}
$$

and 


$$
\gamma_{D M}=\frac{K^{* 2}}{\zeta_{1} / \zeta_{E}+K^{* 2}}
$$

As was shown in Barrero-Gil et al. (2012) the typical behaviour of efficiency in VIV energy extraction is as follows: it becomes higher than zero when the reduced velocity $U^{*}$ is close enough to the resonance condition, that is $U^{*}$ approaching to $2 \pi \mathrm{St}$, St being the Strouhal number ( $\mathrm{St} \approx 0.2$ for a circular cylinder), and increases with $U^{*}$ until a maximum value is achieved. After that, efficiency decreases to zero with $U^{*}$. One of the important issues is the maximum efficiency. This maximum efficiency will be reached without parasite losses, i.e. when $\zeta_{1}=0$. In this case, $\gamma_{D M}=1$, and the maximum efficiency $\eta_{D M}^{M A X}$ depends only on $m_{e q}^{*}$ and $\zeta_{e q}$ since $V^{*}$ disappears as a relevant parameter because the velocity at which $\eta_{D M}^{M A X}$ is achieved is without interest [note that $\left.\chi=\chi\left(m_{e q}^{*}, \zeta_{e q}, V^{*}\right)\right]$. Fig. 5.3 shows $\eta_{D M}^{M A X}$ as a function of the equivalent mechanical properties $m_{e q}^{*}$ and $\zeta_{e q}$ in a contour plot with logarithmic axes. This result has been computed following the iterative procedure described earlier (step 7 is now unnecessary) and for each pair of $m_{e q}^{*}, \zeta_{e q}$, picking the maximum value along $V^{*}$. As it can be checked in the figure, a maximum value of 0.18 is obtained near $m_{e q}^{*} \zeta_{e q}=0.23$. That is, high efficiency is possible for high $m_{e q}^{*}$ when low values of $\zeta_{e q}$ are considered, or with low $m_{e q}^{*}$ and high $\zeta_{e q}$. The reason for which $\eta_{D M}^{M A X}$ is bounded can be explained from Eq. 5.15, where it is seen that $\eta_{D M}$ increases linearly with $m_{e q}^{*} \zeta_{e q}$ and quadratically with $A^{*}$. Since $A^{*}$ decreases with $m_{e q}^{*} \zeta_{e q}$ (see Eq. 5.11b) it follows that an optimal combination of $m_{e q}^{*} \zeta_{e q}$ must exists.

The maximum efficiency obtained can be compared with previous studies. The analytical study carried out by Groutier et al. (2014) employing a wake oscillator model predicts a maximum efficiency of 0.19 which is very close to the value computed here. Raghavan (2007) reported in laboratory experiments efficiencies up to 0.31 , which is higher that the value given here. The discrepancy is probably due to the high Reynolds number regime of their experiments. For other vibration-based solutions proposed, like Transverse Galloping energy harvesting, the theoretical study carried out by Barrero-Gil et al. (2010) predicts a maximum efficiency of 0.54 for a D-type cross-section galloping prism. Finally, in an experimental study of electromagnetic energy extraction using Wake Galloping (not Transverse Galloping) through wind tunnel testing carried out by Jung and Woo-Lee (2011), efficiencies up to 0.094 were measured.

\subsection{Dual-mass versus single-mass configuration}

A discussion on differences between single and dual-mass configurations is presented in order to see if there is a dual-mass configuration which can be advantageous. For the single-mass (SM) configuration $\left(K^{*}=1, \phi=0, \omega_{e q}^{*}=\omega^{*}, m_{e q}^{*}=m^{*}, \zeta_{e q}=\zeta_{1}+\zeta_{E}\right)$ from Eqs. 5.15 and 5.17 one gets

$$
\eta_{S M}=\frac{2 m^{*}\left(\zeta_{1}+\zeta_{E}\right) A^{2} *}{V^{* 3} \omega^{*}} \gamma_{S M}
$$

where 


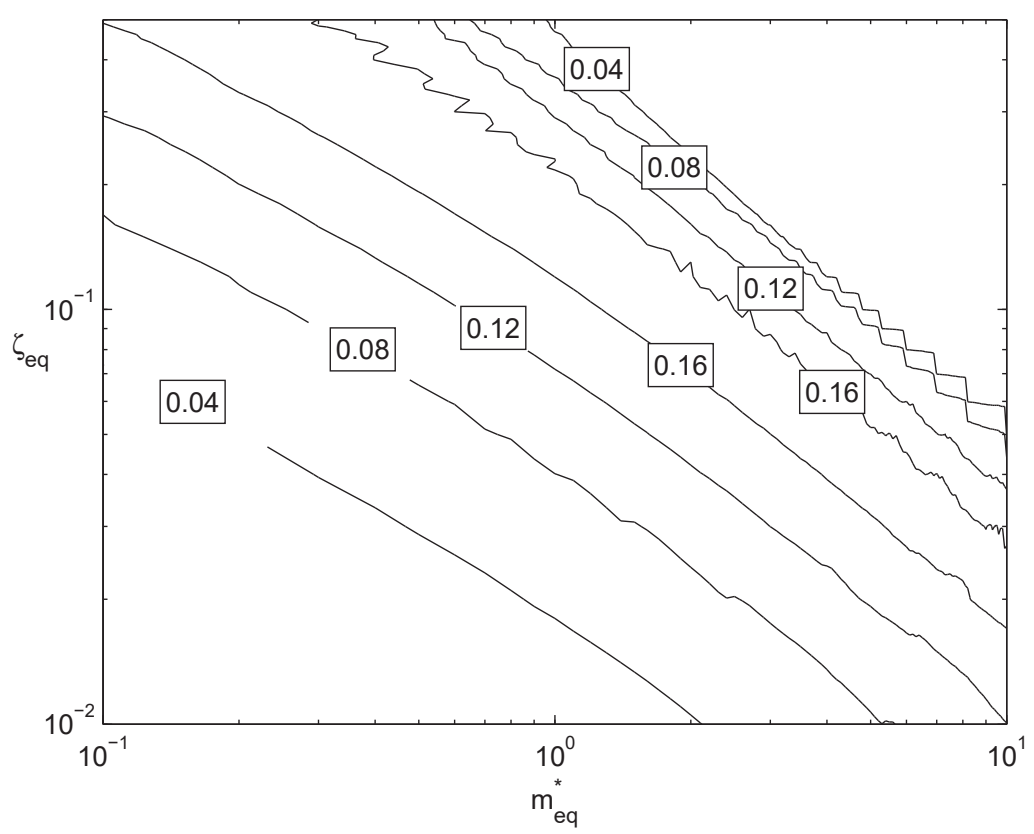

Figure 5.3: Maximum harnessable efficiency for a dual mass system where no dissipation is present $\left(\zeta_{1}=0\right)$ in terms of equivalent damping and mass parameters.

$$
\gamma_{S M}=\frac{1}{\zeta_{1} / \zeta_{E}+1}
$$

and, therefore

$$
\frac{\eta_{D M}^{M A X}}{\eta_{S M}^{M A X}}=\frac{\gamma_{D M}}{\gamma_{S M}}
$$

When parasite damping $\zeta_{1}$ is zero $\gamma_{D M}=\gamma_{S M}$ (see Eq. 5.17) and for equal values of $m_{e q}^{*}$ and $\zeta_{e q}$ it follows $\eta_{D M}^{M A X}=\eta_{S M}^{M A X}$, meaning that, in this case, the use of a DM configuration does not lead to any improvement. This result makes sense, since it can be considered that the SM configuration is just a particular case of the DM one. However, when $\zeta_{1} \neq 0, \gamma_{D M}$ may be higher than $\gamma_{S M}$ meaning that $\eta_{D M}^{M A X}$ can be higher than $\eta_{S M}^{M A X}$. From Eqs. 5.17 and 5.19

$$
\frac{\gamma_{D M}}{\gamma_{S M}}=\frac{K^{* 2}\left(\frac{\zeta_{1}}{\zeta_{E}}+1\right)}{\frac{\zeta_{1}}{\zeta_{E}}+K^{* 2}},
$$

which will be higher than one when $K^{*}>1$ and, therefore $\eta_{D M}^{M A X}>\eta_{S M}^{M A X}$. From the physical point of view, one may see that the power dissipated at each damper is proportional to $\zeta_{E} B^{* 2}$ and $\zeta_{1} A^{* 2}$ respectively so the dual mass system is capable of dissipating the same global power as in the single mass configuration, but proportionally the power dissipated in $c_{E}$ is enhanced when $K^{*}>1$. The potential improvement is larger as $\zeta_{1} / \zeta_{E}$ is increased as can be deduced 
from Eq. 5.21.

Therefore, from the design point of view, in order to maximize efficiency and get an advantage from the dual mass effect, one should choose $\mu$ and $\Omega$ to achieve a value of $K^{*}>1$. Then, the question that naturally arises is how to choose the mechanical parameter $\mu$ and $\Omega$ of the dual mass system.

\subsubsection{Mechanical properties selection of the dual mass system}

It is assumed that $m^{*}$ and $\zeta_{1}$ are constraints of the system before considering the DM improvement. From Fig. 5.3 it may be deduced that the design point should be at $m_{e q}^{*} \zeta_{e q}=0.23$ to maximize the maximum achievable efficiency. In mathematical terms, this is

$$
m_{e q}^{*} \zeta_{e q}=\frac{m^{*} \omega_{e q}^{*}}{\omega^{*}} \zeta_{E}\left(\frac{\zeta_{1}}{\zeta_{E}}+K^{* 2}\right)=0.23
$$

Therefore, when $m^{*}$ and $\zeta_{1}$ are known, Eq. 5.22 allows for the introduction of an optimal relationship between $\zeta_{E}, \omega_{e q}^{*}, K^{*}$ and $\omega^{*}$. In addition, from Eq. 5.10a one may see that $\omega_{e q}^{*}$ depends on $\Omega, K^{*}, \phi$ and $\omega^{*}$, and Eqs. 5.7 and 6.9 establish a relationship between $K^{*}$ and $\phi$ with $\zeta_{E}, \Omega, \mu$ and $\omega^{*}$. Furthermore, $\omega^{*}$ should be computed solving iteratively Eqs. 5.11a and $5.11 \mathrm{~b}$ (note that two modes of oscillation are possible). As a result, it follows that for each pair of $\mu$ and $\Omega, \zeta_{E}$ can be optimally taken and removed as an independent variable (system of equations $5.10 \mathrm{a}, 5.11 \mathrm{a}, 5.11 \mathrm{~b}$ and 5.22 is considered to be solved for that).

Fig. 5.4 shows the optimal value of $\zeta_{E}\left(\zeta_{E}^{O P}\right)$ dependence with $\mu$ and $\Omega$ (note that two possible modes of steady oscillations are possible) for fixed values $m^{*}=2$ and $\zeta_{1}=0.01$. The dependence is dramatically different for each mode. When $\omega^{*}>1, \zeta_{E}^{O P}$ grows with $\mu$ whereas the dependence with $\Omega$ is not very strong. Conversely, when $\omega^{*}<1, \zeta_{E}^{O P}$ diminishes with $\mu$ and grows with $\Omega$. In addition, as can be seen in the figure, solution $\omega^{*}<1$ induces low values of $\zeta_{E}^{O P}$, so that it is expected that this solution will be less practical (in other words, it will be only of interest for cases with small energy harvesting).

Fig. 5.5 shows $K^{*}$ variation with $\mu$ and $\Omega$ for each mode (for fixed values $m^{*}=2$ and $\zeta_{1}=0.01$ ). For $\omega^{*}>1$ (Fig. 5.5a) the region of interest for energy harvesting purposes $\left(K^{*}>1\right)$ is restricted to values of $\mu<1$. As can be seen, it is possible to have large $K^{*}$ values for low $\mu$ values (the secondary mass is small compared to the cylinder mass). The dependence with $\Omega$ is weak except when $\Omega$ is low (say $\Omega<1$ ). For the other mode of oscillation, when $\omega^{*}<1$ (Fig. $5.5 \mathrm{~b})$, the dependence with $\mu$ is weak and large values of $K^{*}$ can be achieved for low values of $\Omega$.

For completeness, Fig. 5.6 shows, for mode $\omega^{*}>1, K^{*}$ s behaviour in the interesting zone from the energy harvesting point of view (that is, $K^{*}>1$ ) superimposed with isolines with $\zeta_{E}^{O P}$. This figure can be considered as a design chart since it allows to design the dual mass system (that is, $\mu$ and $\Omega$ ) to achieve a certain level of improvement $\left(K^{*}\right.$ value and thus a $\gamma_{D M} / \gamma_{S M}$ value) using $\zeta_{E}^{O P}$ for each design point. From the practical side it should be noted that the level of mechanical damping introduced by an electromagnetic generator can be tuned by choosing 

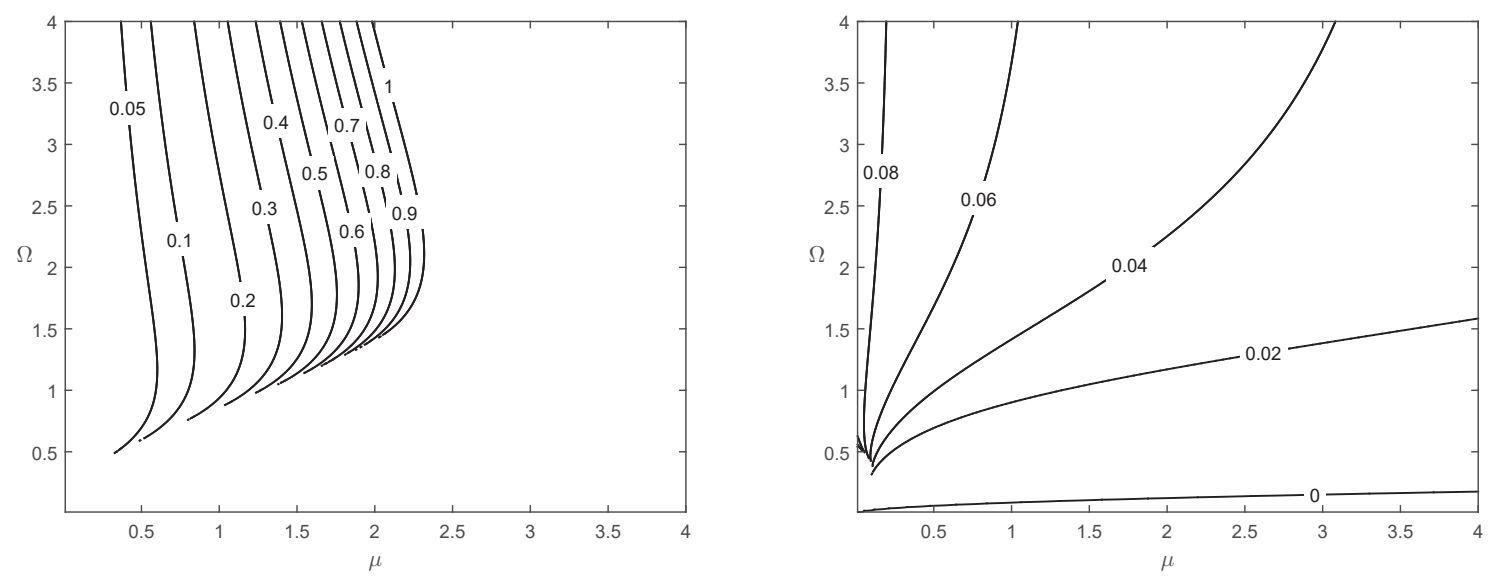

Figure 5.4: Optimal $\zeta_{E}$ (electric damping) dependence with $\mu$ and $\Omega$ for (a) $\omega^{*}>1$ and (b) $\omega^{*}<1 \cdot m^{*}=2, \zeta_{1}=0.01$.

appropriately the electrical load resistance (Cammarano et al. 2010).

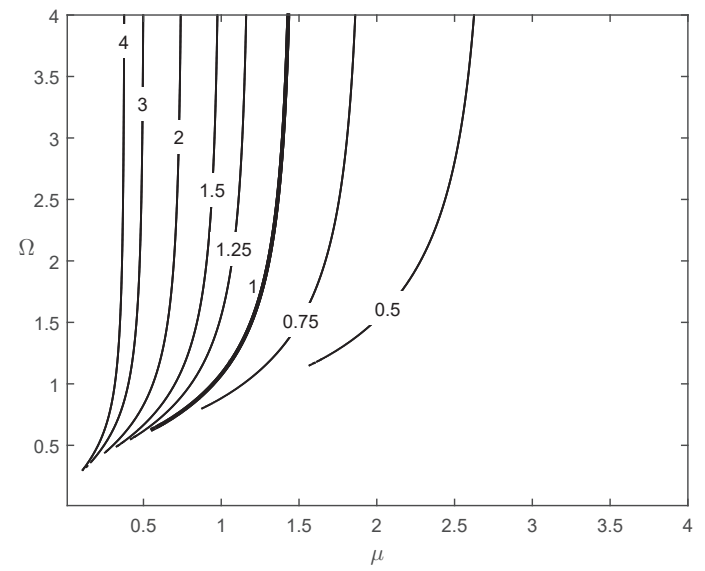

(a)

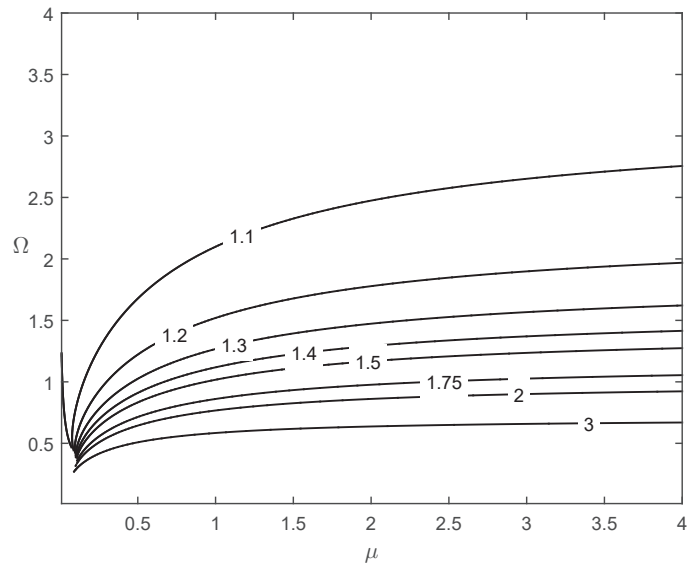

(b)

Figure 5.5: Amplitude ratio $K^{*}$ contour plot over $\mu$ and $\Omega$ for (a) $\omega^{*}>1$ and (b) $\omega^{*}<1$. $m^{*}=2, \zeta_{1}=0.01$.

\subsection{Application example}

As an example of the dual-mass effect and its potential improvement with respect to the single mass configuration, an illustrative reconfiguration is presented in this Section. First of all, a single mass configuration is considered whose mass ratio is fixed at a value of $m^{*}=2$, which can be considered as a representative case where the circular cylinder, whose mass, 


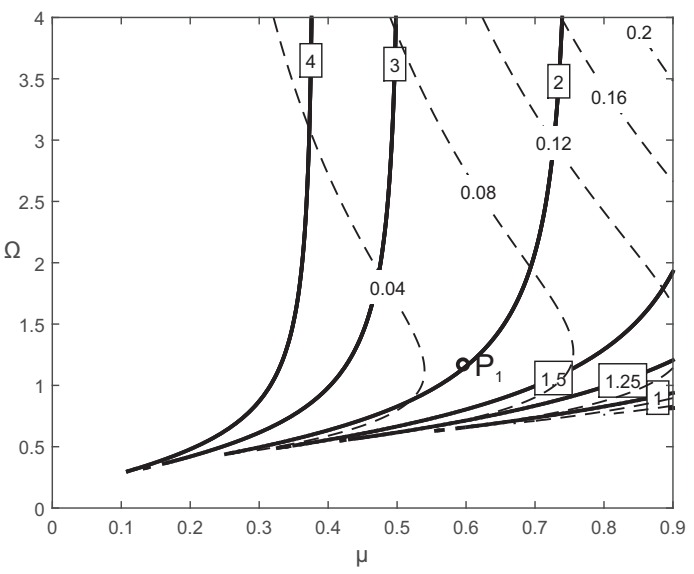

(a)

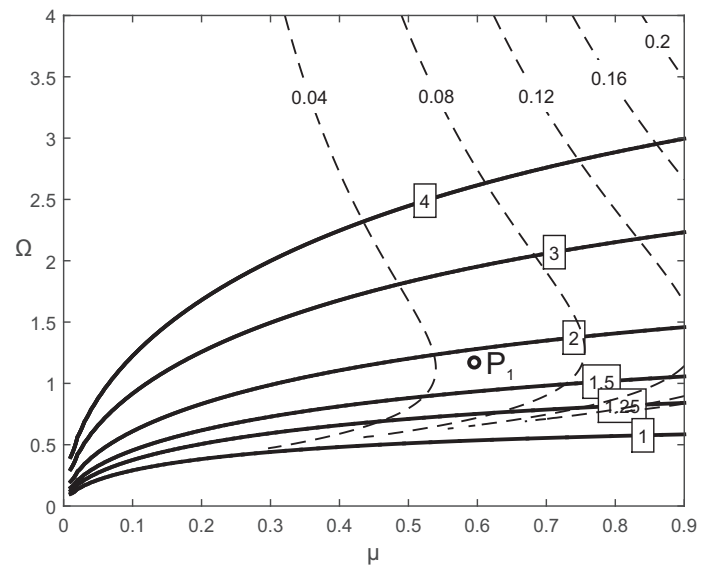

(b)

Figure 5.6: Detail of $K^{*}$ (a) and $\omega^{*}$ (b) dependence with $\mu$ and $\omega$ in the zone of interest $\left(\omega^{*}>1\right)$. It is superimposed by dashed isolines with optimal $\zeta_{E} \cdot m^{*}=2, \zeta_{1}=0.01$.

diameter and submerged length are $160 \mathrm{Kg}, 0.2 \mathrm{~m}$ and $2 \mathrm{~m}$ respectively, is immersed in a dense fluid. Please note that $m^{*}$ in single mass configurations is defined in the same way as dual mass configurations, where $m$ is the mass of the single cylinder and $\rho D L$ is the mass of the displaced fluid by the cylinder. The mechanical loss in the guide where the system is mounted is characterized as $\zeta_{1}=0.01$, and the electrical damping is assumed to be $\zeta_{E}=0.042$. For this case, the efficiency variation with $U^{*}$ is shown in Fig. 5.7. The maximum efficiency achieved is 0.113 close to $U^{*}=0.74$. If the example stream's design speed was $U=0.5 \mathrm{~m} / \mathrm{s}$, the spring constant that gives natural frequency of $3.4 \mathrm{rad} / \mathrm{s}$ should be $1847 \mathrm{~N} / \mathrm{m}$.

In order to improve the maximum efficiency and make the energy harvester operate in a larger range of velocities using a dual-mass system, $K^{*}$ must be larger than one as said earlier. The single mass base system's parameters are invariable $\left(k_{1}, m_{1}, c_{1}\left(\zeta_{1}\right), \zeta_{E}, D\right.$ and $\left.L\right)$. A priori, there are two regions of possible design point, one for each synchronized $\omega^{*}\left(\omega^{*}<1\right.$ for designing flow velocities higher than $0.5 \mathrm{~m} / \mathrm{s}$ or $\omega^{*}>1$ for those lower than this). Therefore, if the case where $\omega^{*}>1$ is selected, from Fig. 5.6, a possible design point $P_{1}$ is chosen with $\mu=0.6, \Omega=1.14$, which gives $\zeta_{E}=0.042$ and $K^{*}=1.72$ (in dimensional terms: $m_{2}=96$ $\mathrm{Kg}, k_{2}=2400 \mathrm{~N} / \mathrm{m}$ ). Fig. 5.7 shows the efficiency dependence with $U^{*}$ of the dual mass in this case. As can be seen, the maximum achievable efficiency is now 0.173 , which is more than fifty per cent higher than the case of single mass configuration. Fig. 5.8 shows the normalized amplitude of oscillations variation with the reduced velocity for the single mass configurations as well as for the dual mass one. Note that in the dual mass case, $B^{*}$ is larger than $A^{*}$. That is the reason why the efficiency improves in this dual mass design.

Another beneficial effect of the dual mass system can be noted, since the efficiency behavior is more broadband. The dual mass system enlarges the range of $U^{*}$ where VIV efficiency is higher than zero. In the single mass configuration, the region of positive efficiency is given in the range $0.6<U^{*}<1.3\left(\Delta U^{*}=0.7\right)$, whereas in the dual mass system, the region of positive efficiency and amplitude are given in the range $1.4<U^{*}<2.6\left(\Delta U^{*}=1.2\right)$. The applicable 
range improves which was anticipated by Fig. $5.6 \mathrm{~b}$, where for the design point $(\mu=0.6$, $\Omega=1.14$ ) one may check that $\omega^{*}=1.78$. VIV synchronization occurs when vortex shedding frequency approaches the system natural frequencies, which takes place when $V^{*}$ is inside the interval $2 \pi \mathrm{St}-\Delta V^{*}<V^{*}<2 \pi \mathrm{St}+\Delta V^{*}$, where $\Delta V^{*}$ is mainly influenced by the mass ration being larger for lower $m^{*}$. Since $V^{*}=U^{*} / \omega^{*}$ it follows that, in terms of the reduced velocity, the expected range of synchronization should be $2 \pi \mathrm{St} \omega^{*}-\Delta V^{*} \omega^{*}<U^{*}<2 \pi \mathrm{St} \omega^{*}+\Delta V^{*} \omega^{*}$. It is interesting to observe that this opens the possibility for designing following two different strategies, with the main aim to increase the maximum efficiency (that is to get $K^{*}$ as high as possible) or with the aim to have a broadband character (that is to get $\omega^{*}$ as high as possible).

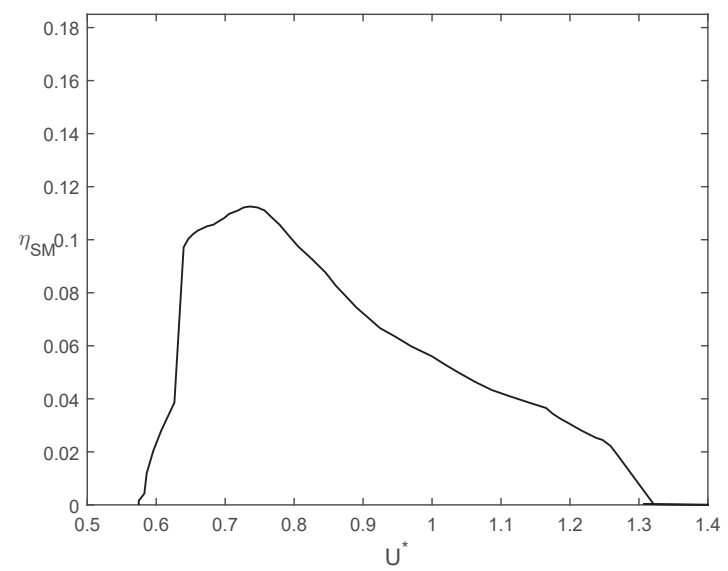

(a)

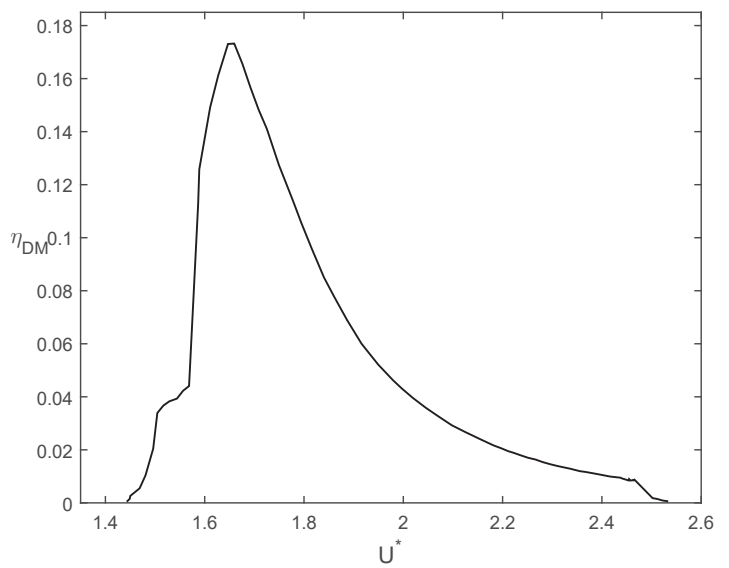

(b)

Figure 5.7: Efficiency as a function of the reduced velocity for the single mass configuration (a) and the dual mass configuration (b).

In this example, the single mass device extracts energy from a $0.5 \mathrm{~m} / \mathrm{s}$ water stream. If a dual mass subsystem is added to the single mass one, efficiency will be increased, however, $U^{*}$ increases as well. Dual mass system has its maximum efficiency in $U=1.1 \mathrm{~m} / \mathrm{s}$. This displacement must appear in a reconfiguration with a dual mass, as the system natural frequency is changed. The efficiency increases due to the optimal $\zeta_{E}^{O P}$ obtained by Eq. 5.22 and $\gamma_{D M}$, setting $K^{*}$ greater than unity when designing.

\subsection{Conclusions}

This study has focused on the effect of a dual-mass system taking into account the efficiency of energy extraction of a fluid flow from VIV. From a fundamental point of view, and specifically related to the questions addressed throughout the Introduction Section, the analysis have shown that (i) in an ideal situation (when $\zeta_{1}=0$ ) the theoretical maximum efficiency can not be enhanced by using a dual-mass system, (ii) when $\zeta_{1} \neq 0$, the maximum efficiency with respect to the single mass configuration can be improved if the dual mass parameters, namely the secondary mass $(\mu)$ and the stiffness between masses $(\Omega)$ are chosen appropriately, (iii) the 


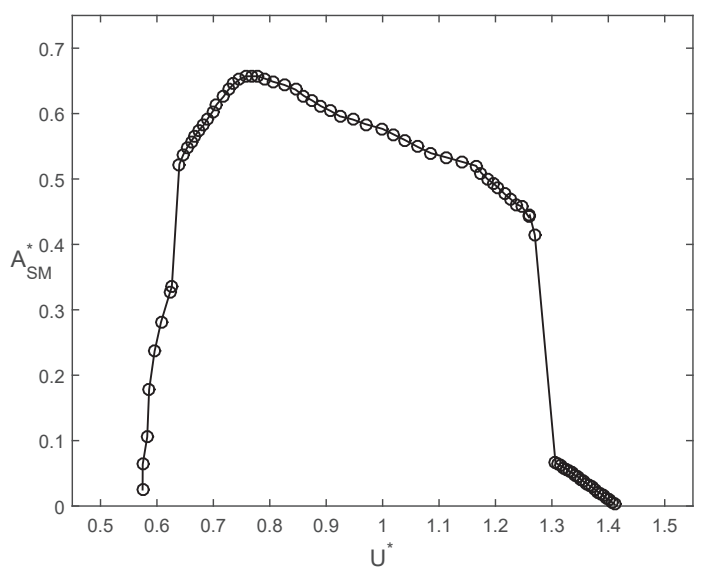

(a)

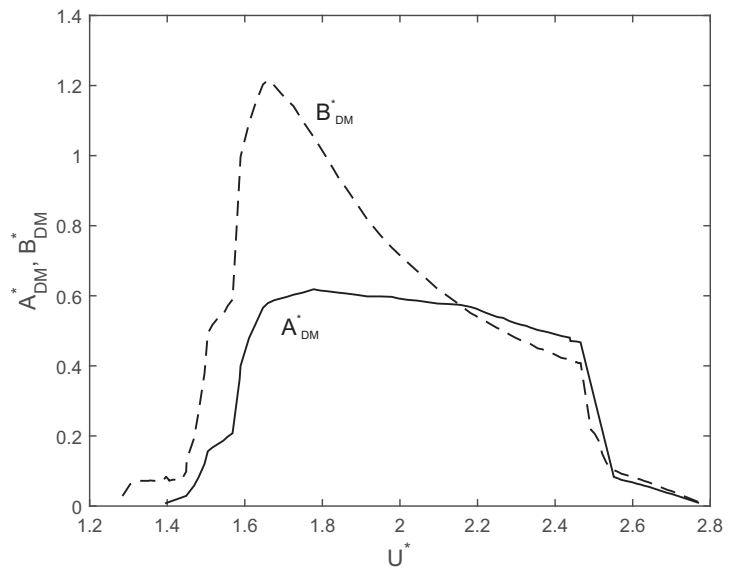

(b)

Figure 5.8: (a) Normalized amplitude of oscillations variation with the reduced velocity for the single mass configuration and (b) for the dual mass one (remember that $A^{*}$ stands for the circular cylinder and $B^{*}$ for the dual mass).

dual-mass system has a more broadband response, which can be of practical relevance in real flows where fluid flow velocity is not constant.

From a fundamental point of view, the analysis carried out sheds light onto the mechanism by which the dual mass effect can be beneficial. The key idea is that, under equivalent configurations, the overall power dissipated is the same in the dual mass configuration as well as in the single mass one but, when properly designed, the power dissipated at the generator in the dual mass configuration is proportionally increased with respect to the power dissipated by mechanical losses, since oscillations of the dual mass are enhanced with respect to the oscillations of the main body.

From a practical point of view, the analysis provides design charts to select dual-mass parameter, namely $\mu$ and $\Omega$ to take advantage of the dual-mass effect, which can be useful to design a dual mass system to extract energy more efficiently from a surrounding flow by VIV of a circular cylinder. Finally, it must be noted however, that the analysis carried out should be seen as an approximation to the actual problem. For example, the real VIV situation is more complex than that of controlled forced vibrations at fixed Reynolds number, and the question of the level of accuracy achieved when results from forced vibration tests are used is still an open question (see Morse and Williamson, 2009). Nevertheless, the results presented in this Chapter are believed to be representative and helpful to design a device to extract energy from VIV in a efficient way. 


\section{Nomenclature}

\section{Acronyms}

DM Dual mass

SM Single mass

VIV Vortex induced vibration

\section{Latin symbols}

A Amplitude of VIV cylinder oscillation

$A^{*} \quad$ Dimensionless amplitude of VIV cylinder oscillation

$B \quad$ Amplitude of second mass oscillation

$B^{*} \quad$ Dimensionless amplitude of second mass oscillation

$c_{1} \quad$ Damping quantity of the VIV cylinder ground clamping

$c_{E} \quad$ Equivalent damping of the electric generator

$c_{L a} \quad$ Force coefficient in phase with acceleration

$c_{L v} \quad$ Force coefficient in phase with velocity

D Circular cylinder diameter

F Overall fluid force in the cylinder

$k_{1} \quad$ Spring rigid constant of the VIV cylinder respect to the ground

$k_{2} \quad$ Spring rigid constant of the second mass respect to the first mass

$K^{*} \quad$ Ratio of amplitudes between two masses

$L \quad$ cylinder length

$m_{1} \quad$ Mass of the cylinder under VIV

$m_{2} \quad$ Mass of the second mass

$m^{*} \quad$ Dimensionless mass of the cylinder under VIV

$m_{e q}^{*} \quad$ Equivalent mass relating SM and DM problem

$t$ Time 
$T$ Integral averaging time

$U \quad$ Non disturbed inflow velocity

$U^{*} \quad$ Reduced/dimensionless velocity with the natural frequency of the primary mass

$V^{*} \quad$ Reduced/dimensionless velocity with the actual frequency of the cylinder movement

$y_{1} \quad$ Displacement of the VIV cylinder

$y_{2} \quad$ Displacement of the second mass

$\dot{y_{1}} \quad$ Velocity of the VIV cylinder

$\dot{y}_{2} \quad$ Velocity of the second mass

$\ddot{y_{1}} \quad$ Acceleration of the VIV cylinder

$\ddot{y_{2}} \quad$ Acceleration of the second mass

\section{Greek symbols}

$\gamma \quad$ Efficiency coefficient

$\zeta_{1}$ Dimensionless damping coefficient of the VIV cylinder ground clamping

$\zeta_{E} \quad$ Dimensionless equivalent damping coefficient of the electrical generator

$\zeta_{e} q \quad$ Equivalent damping coefficient relating SM and DM problem

$\eta \quad$ Efficiency

$\eta_{1} \quad$ Dimensionless displacement of the VIV cylinder

$\eta_{2} \quad$ Dimensionless displacement of the second mass

$\mu \quad$ Ratio between two masses (VIV cylinder and second mass)

$\rho \quad$ Fluid density

$\tau$ Dimensionless time

$\phi \quad$ Phase delay between VIV cylinder and second mass

$\chi \quad$ Second efficiency coefficient

$\Omega \quad$ Square ratio between rigid constants

$\omega$ Actual frequency of the cylinder movement

$\omega_{1} \quad$ Single mass natural frequency 
$\omega^{*} \quad$ Dimensionless frequency

$\omega_{e q}^{*} \quad$ Equivalent dimensionless frequency relating SM and DM problem 


\section{Chapter 6}

\section{A VIV based electromagnetic energy harvester}

\subsection{Introduction}

During the past few years, energy harvesting from vibration sources has received a significant amount of attention. The reasons might be twofold: the presence of vibration sources is ubiquitous and many different options exist to transform the mechanical energy of vibrations into electrical energy. When looking at the specialized literature, it could be observed that two main sources of vibration are typically considered: those deriving from mechanical sources and those deriving from some kind of fluid-structure interaction. Regarding the first class, it is worth mentioning the works of Yuan et al. (2014) that considered railroad track vibration, Mitcheson et al. (2004a) that accounted for human powered and machine powered vibrations, Von Büren et al. (2006) that also considered energy coming from human motion, Galchev et al. (2011) that analysed applications involving low frequency machinery vibrations, and Deng et al. (2014) and Adhikari et al. (2009) that considered weak broadband vibration sources. Regarding the second class, vibrations originated from fluid-structure interactions, they have been studied by many authors. Although it sometimes happens that the nature of the interaction itself cannot be ascribed undoubtedly to a single regime, it is useful, for the sake of clarity, to refer to well defined regimes. Specifically, in this short review, galloping, vortex induced vibration (VIV) and flutter will be the types of instabilities/resonances to be considered. Energy extraction from the galloping phenomenon has been studied recently by A. Barrero-Gil et al. (2010), Abdelkefi et al. (2013a, 2013b), Jung and Lee (2011) and Vicente-Ludlam et al. (2014, 2015). The use of VIV to harvest energy has been analysed, among others, by Sanchez-Sanz et al. (2009), Barrero-Gil et al. (2012), Weinstein et al. (2012), Nishi (2013), Lee and Bernitsas (2011), and Wu et al. (2013). The potential of flutter type vibrations for energy harvesting purposes has been studied by Doaré and Michelin (2011), Tang et al. (2009), Zhu (2012) and Allen and Smith (2001).

Regarding the mechanical to electrical conversion system, the three most commonly used approaches are the piezoelectric, the electromagnetic and the capacitive micropower generators. For a comprehensive review on piezoelectric based generators for energy harvesting purposes, the reader is referred to the article by Saadon and Sidek (2011) and to the references therein. Among the very large number of related articles published in the specialized literature, it is also worth mentioning the the generic analysis published by Liang and Liao (2011) and the novel approaches proposed by Hobeck and Inman (2012) and Zhou et al. (2012). While the compactness of piezoelectric generators makes them useful for MEMS based energy harvesting systems, in the mini scale, with characteristic dimensions of the order of millimeters/centimeters, electromagnetic micro power generators appear to be a satisfactory compromise between complexity, size and conversion efficiency. In this field many studies have been reported in the literature 
and, among them, it is relevant to refer to the works of Mitcheson et al. (2004a), Burrow and Penrose (2014), Sari et al. (2010), and Wang et al. (2007). Finally, regarding capacitive generators, the reader is directed to the articles published by Peano and Tambosso (2005) and Mitcheson et al. (2004b).

The objective of this article is to present a theoretical study oriented towards the design of an efficient coupling between a VIV cylindrical resonator and a electromagnetic power generator. The rationale for the study is that, in this case, the presence of the generator, that is modeled as a secondary mass with some associated damping and stiffness, influences the motion (and the energy conversion characteristics) of the resonator itself. This is in contrast, for instance, with some examples mentioned in Mitcheson et al. (2004a) related to energy harvesting from human motion. In this other case, the energy source and the energy harvester are of such disparity that their interaction is a one-way interaction. However, in the case considered in this article the harvester influences the resonator and the resonator influences the harvester, so their interaction is a two-way interaction.

In a sense, a two-masses modeling of the coupling between a VIV resonator and an electromagnetic generator resembles, from the mathematical modeling standpoint, the configuration of a dual-mass system for energy harvesting purposes. This type of dual-mass configuration has been recently discussed at length by Burrow and Penrose (2014), Nishi (2013) and Tang and Zuo (2011). The study presented by Nishi (2013) is relevant to the present article because it examines theoretically energy extraction via VIV of a dual mass system. However there are three main differences between the study of Nishi (2013) and the present study: a) Nishi considered a wake oscillator approximation for the motion of the two masses while in the present study a forcing model, Païdoussis et al. (2011), has been implemented (the coefficients needed for the model were obtained after the experimental results published by Hover et al. 1998); b) the system configuration in Nishi (2013) considered each of the two masses attached to a fixed wall while in the present study the secondary mass is attached to the primary mass (mimicking a practical engineering application); c) the propose of the work of Nishi (2013) is to show that a dual mass configuration may increase the amount of harvested energy while the objective of the present study is to optimize the coupling between the generator and the VIV resonator. Finally, the work of Tang and Zuo (2011) also concentrates on showing the superiority of dual-mass systems for energy harvesting purposes. However they consider a prescribed base vibration (not coming from a fluid structure interaction situation) that is not affected by the dynamics of the motion of the two masses which is not what happens when fluid structure interaction is the origin of the vibration (like in the present article).

Regarding the organization of this Chapter, the concept and its basic geometry are presented first. Then, the model equations and their hypothesis are described and are followed by the solution method. Next, results are presented and discussed, and conclusions and practical design guidelines are given. 


\subsection{Description of the concept}

The basic concept, as sketched in Figure 6.1, consists of a cylindrical resonator attached to a supporting cantilever. When the working fluid flows past the resonator with the appropriate conditions, large vortices are shed, unsteady forces appear on the cylindrical body, and the cylinder starts to move harmonically around its equilibrium position.
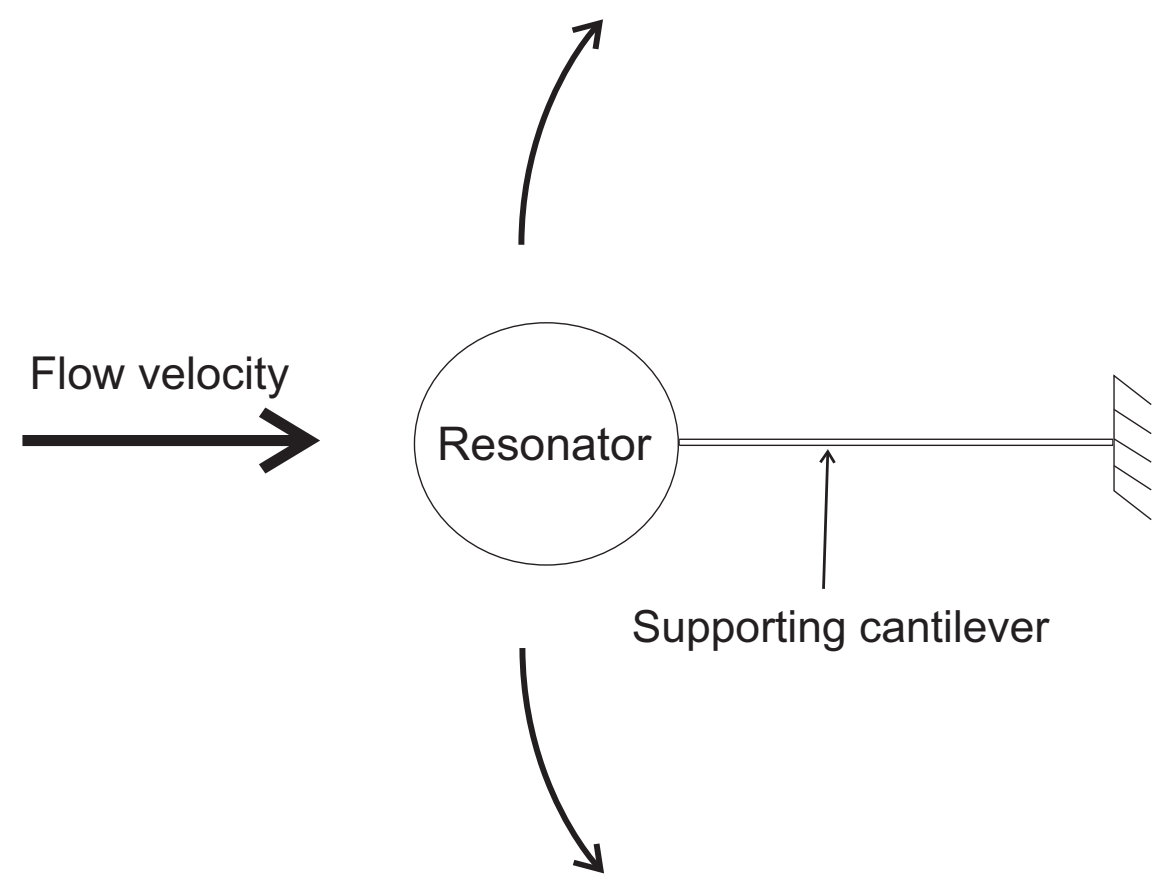

Supporting cantilever

Figure 6.1: Top view of the configuration being considered.

To transform this motion into electrical energy, a permanent magnet electromagnetic generator is chosen. In a sketchy way, this generator is made up of a stator and translator part (the equivalent of the rotor in a conventional generator). It is the magnetic field variation caused by the linear motion of the translator (see Figure 6.2) what converts mechanical energy into electric energy. Details of this type of design have been reported by Mitcheson et al. (2004a) and von Büren and Tröster (2007). Because of compact design considerations, this generator is placed on top of the resonator. Additionally, another advantage of this configuration is that, if a water current is chosen, the generator and the supporting cantilever are located above the water surface while it is only the cylindrical resonator the element that is submerged. In practical terms, the masses of the stator and the translator are very similar for most of the designs presented in the literature. Since a typical cylinder would be very light, manufactured, for instance out of plastic, the mass of the main resonator can be approximated as the stator mass. A practical realization of the concept could be, for example, the one shown in Figure 6.3. In this set-up, the primary resonator is defined by its cross section shape, its length $\mathrm{L}$ and diameter $\mathrm{D}$, its mass $m_{1}$ and the stiffness $k_{1}$ and damping $c_{1}$ of the supporting cantilever. The translator part of the electromagnetic generator is defined by its mass $m_{2}$, and associated stiffness and damping $k_{2}$ and $c_{2}$.

Anticipating results that will be obtained in another section, in a conventional application 


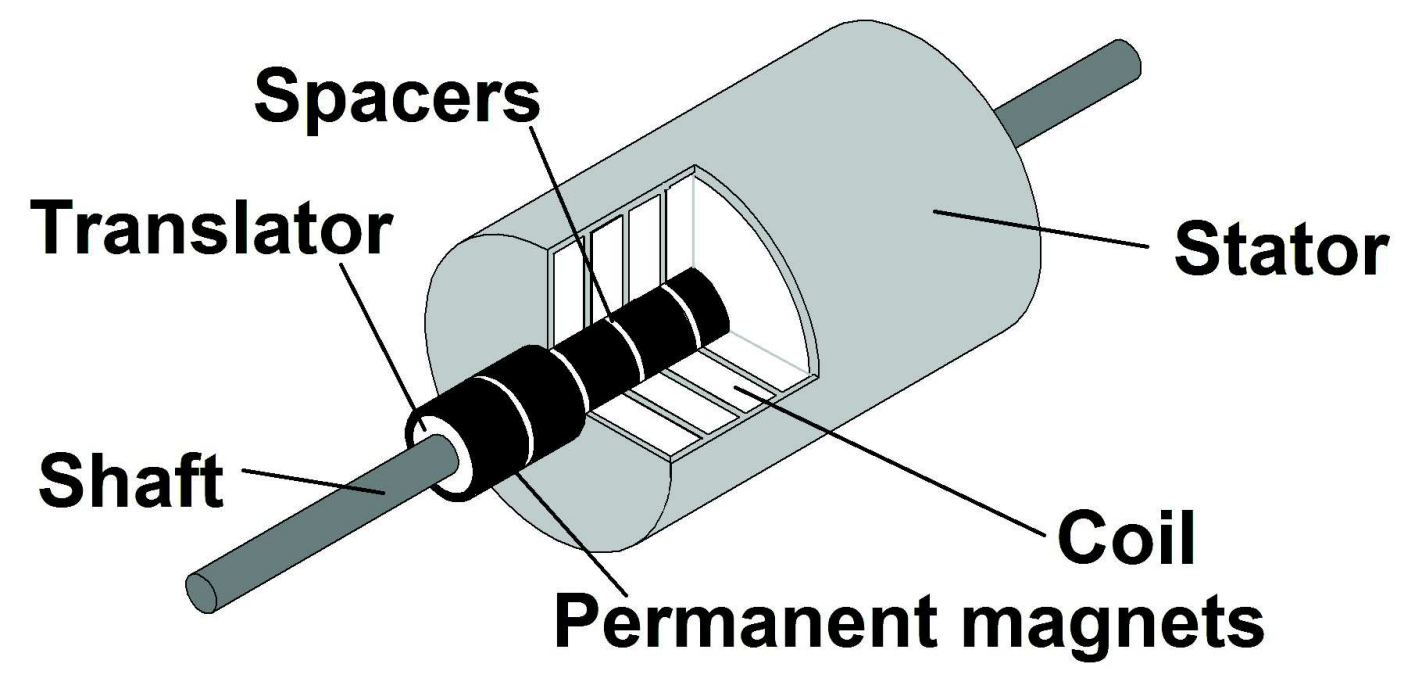

Figure 6.2: Sketch of a linear vibration driven electromagnetic generator.

for a water current flowing at $0.5 \mathrm{~m} / \mathrm{s}$ that may represent a small stream, a practical system could be as follows:

- Resonator diameter, length and mass: $2 \mathrm{~cm}, 10 \mathrm{~cm}$ and 100 gr respectively (manufactured in plastic).

- Supporting cantilever dimensions, stiffness and damping: $100 \mathrm{~mm}$ x $20 \mathrm{~mm}$ x $1 \mathrm{~mm}, 295$ $\mathrm{N} / \mathrm{m}$ and $0.022 \mathrm{Ns} / \mathrm{m}$ respectively (manufactured in aluminium).

In these conditions the power obtained at the electromagnetic generator would be $22 \mathrm{~mW}$. The peak to peak amplitude of the motion of the resonator would be $2.3 \mathrm{~cm}$. The conversion efficiency (defined as the output electric power divided by the incoming flow kinetic energy per unit time) is $8 \%$. Note that this efficiency is defined accounting for the kinetic energy contained in a fluid volume whose width is the peak to peak amplitude of the resonator motion, as it is customary in engineering applications. This is in contrast to some other theoretical studies in which the efficiency is defined using the resonator diameter as the characteristic length. If this alternative way of defining efficiency is used, its computed value would be $18 \%$.

\subsection{Mathematical model}

The model scheme associated to the possible practical application is shown in Figure 6.4. The primary resonator is defined by its mass $m_{1}$, flexural stiffness $k_{1}$ and flexural damping $c_{1}$, the secondary resonator is defined by its mass $m_{2}$, stiffness $k_{2}$ and damping $c_{2}$.

Since the oscillation half-amplitude is significantly smaller than the supporting cantilever length, it will be assumed that the motion is one dimensional in the y direction (see Figure 


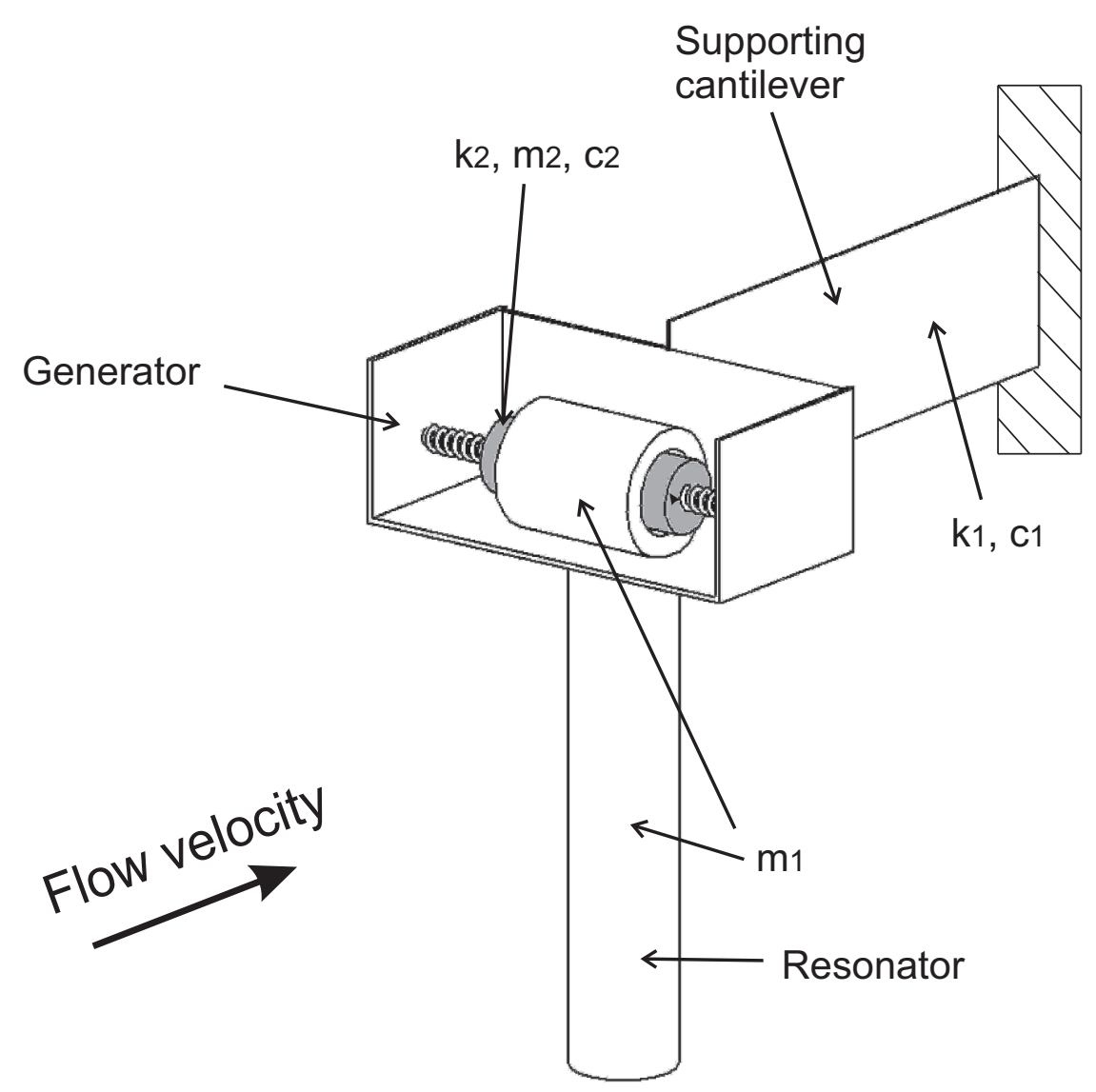

Figure 6.3: Sketch of a possible practical realization.

6.4). Note that, in the potential practical application presented in Section 6.2, the supporting cantilever length was $10 \mathrm{~cm}$ and the oscillation half-amplitude $1.16 \mathrm{~cm}$. Defining the absolute motion coordinates of the resonator and the generator $y_{1}$ and $y_{2}$ respectively, the two Equations of motion of the problem for the two masses $m_{1}$ and $m_{2}$ are:

$$
\begin{gathered}
m_{1} \ddot{y}_{1}+c_{1} \dot{y}_{1}+k_{1} y_{1}-c_{2}\left(\dot{y}_{2}-\dot{y}_{1}\right)-k_{2}\left(y_{2}-y_{1}\right)=F_{f} \\
m_{2} \ddot{y}_{2}+c_{2}\left(\dot{y}_{2}-\dot{y}_{1}\right)+k_{2}\left(y_{2}-y_{1}\right)=0 .
\end{gathered}
$$

Fluid forces, $F_{f}$, in Equation 6.1, that are related to vortex shedding, can be modeled by different approximations. For a very comprehensive review on the subject, the reader is directed to the monograph by Paidoussis et al. (2011). In the present work, the so called "Advanced forcing model" has been chosen (see Section 3.3.3 (b) of Paidoussis et al. 2011). The reason is that this model is, on one hand, rather simple and, therefore, suitable for a theoretical analysis and, on the other, lift coefficients needed to close the model are taken from already published 
(a)

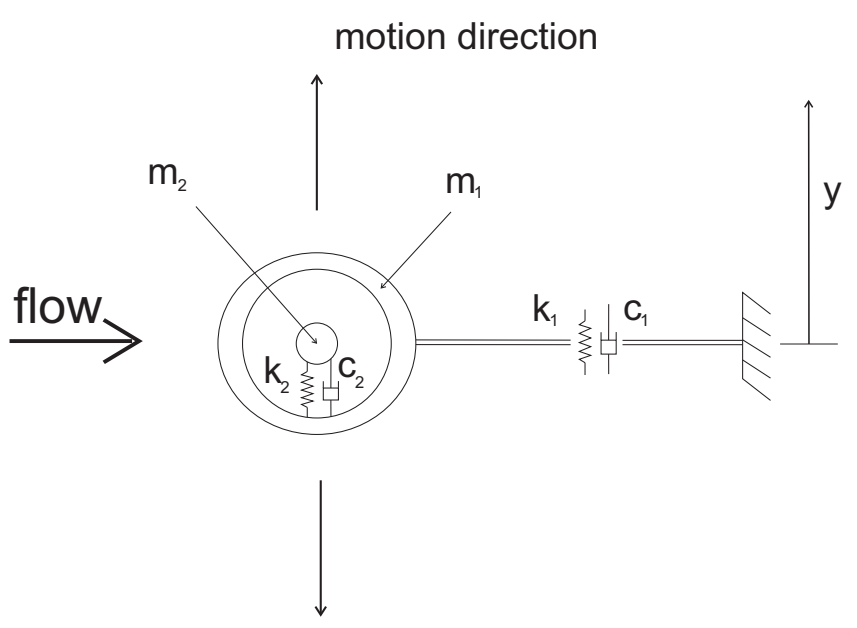

(b)

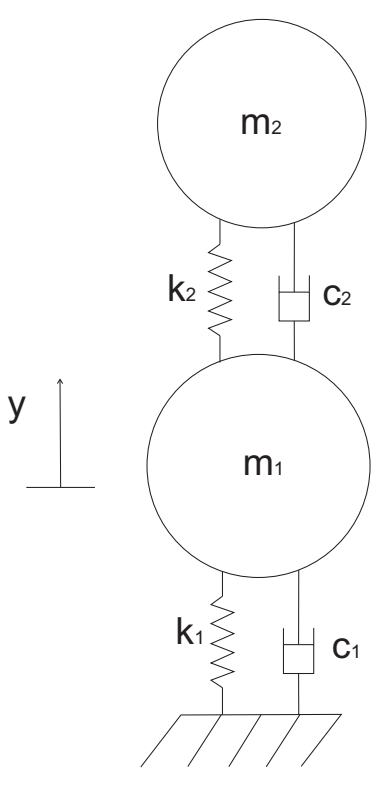

Figure 6.4: Model scheme.

experimental data, which guarantees a reasonable accuracy of the model application. In this work, the experimental results of Hover et al. (1988) have been selected for this particular modelling purpose which yields:

$$
F_{f}=\frac{1}{2} \rho_{\infty} D L U_{\infty}^{2}\left(-c_{L a} \sin (\omega t)+c_{L v} \cos (\omega t)\right) .
$$

Where $\rho_{\infty}$ and $U_{\infty}$ are the fluid density and velocity, D and $\mathrm{L}$ the resonator diameter and length respectively. $c_{L a}$ and $c_{L v}$ depend on $A / D$ and $U /(D \omega)$ where $A$ and $\omega$ are the oscillation amplitude and frequency respectively. That is the fluid force in this modes depends on the motion itself which suggests that the problem has to be solved in an iterative way until solution of Equations 6.1-6.2 yields a motion amplitude and frequency consistent with the fluid force 6.3. The actual plots of $c_{L a}$ and $c_{L v}$ that have been used can be seen in Figures 12 (page 109) and 14 (page 110) of Hover et al. (1988).

Since only the steady-state solutions of Equations 6.1-6.2 will be sought no initial conditions have to be prescribed for $y_{1}, \dot{y}_{1}, y_{2}$ and $\dot{y}_{2}$.

\subsection{Solution method}

The first step is to re-write Equations 6.1-6.2 in dimensionless form. To this end, the following dimensionless variables and parameters are defined, together with a characteristic frequency $\omega_{N 1}=\left(\frac{k_{1}}{m_{1}}\right)^{1 / 2}$ : 
$\quad \eta_{1}=\frac{y_{1}}{D}, \eta_{2}=\frac{y_{2}}{D}, \tau=t \omega_{N 1}, m^{*}=\frac{m_{1}}{\rho_{\infty} D^{2} L}, \mu=\frac{m_{2}}{m_{1}}, \omega^{*}=\frac{\omega}{\omega_{N 1}}, U^{*}=\frac{U_{\infty}}{\omega_{N 1} D}, \Omega=\left(\frac{k_{2}}{k_{1}}\right)^{1 / 2}$,
$\zeta_{1}=\frac{c_{1}}{2 \omega_{N 1} m 1}, \zeta_{2}=\frac{c_{2}}{2 \omega_{N 1} m 1}$.

In this way, considering apostrophe $\left({ }^{\prime}\right)$ as derivation respect to the dimensionless time $\tau$, Equations 6.1-6.2 are re-written as:

$$
\begin{gathered}
\eta_{1}^{\prime \prime}+2 \zeta_{1} \eta_{1}^{\prime}+\eta_{1}-2 \zeta_{2}\left(\eta_{2}^{\prime}-\eta_{1}^{\prime}\right)-\Omega^{2}\left(\eta_{2}-\eta_{1}\right)=\frac{1}{2} \frac{U^{* 2}}{m^{*}}\left(-c_{L a} \sin \left(\omega^{*} \tau\right)+c_{L v} \cos \left(\omega^{*} \tau\right)\right) \\
\mu \eta_{2}^{\prime \prime}+2 \zeta_{2}\left(\eta_{2}^{\prime}-\eta_{1}^{\prime}\right)+\Omega^{2}\left(\eta_{2}-\eta_{1}\right)=0
\end{gathered}
$$

Assuming, as experimental evidence attests, that the steady state motion is harmonic, the dimensionless amplitudes $\eta_{1}$ and $\eta_{2}$ can be written as:

$$
\eta_{1}=A^{*} \sin \left(\omega^{*} \tau\right), \eta_{2}=B^{*} \sin \left(\omega^{*} \tau+\phi\right)
$$

Where $A^{*}$ and $B^{*}$ are the dimensionless motion amplitudes to be determined, and $\phi$ is the phase lag also to be determined. Introducing relations 6.6 into Equations 6.4-6.5, the use of the harmonic balance method yields a set of four equations as follows:

$$
\begin{gathered}
A^{*}\left(1-\omega^{* 2}+\Omega^{2}\left(1-\frac{B^{*}}{A^{*}} \cos \varphi\right) 2 \frac{B^{*}}{A^{*}} \omega^{*} \zeta_{2} \sin \varphi\right)=-\frac{T^{2} *}{2 m^{*}} c_{L a}, \\
2 A^{*} \omega^{*}\left(\zeta_{1}+\zeta_{2}\left(1-\frac{B^{*}}{A^{*}} \cos \varphi\right)-\frac{\Omega^{2}}{2 \omega^{*}} \frac{B^{*}}{A^{*}} \sin \varphi\right)=\frac{T^{* 2}}{2 m^{*}} c L v, \\
\frac{B^{*}}{A^{*}}\left(\left(\Omega^{2}-\mu \Omega^{* 2}\right) \cos \varphi-2 \omega^{*} \zeta_{2} \sin \varphi\right)-\Omega^{2}=0, \\
\frac{B^{*}}{A^{*}}\left(\left(\Omega^{2}-\mu \Omega^{* 2}\right) \sin \varphi+2 \omega^{*} \zeta_{2} \cos \varphi\right)-2 \omega^{*} \zeta_{2}=0 .
\end{gathered}
$$

Note that the number of unknowns is four: the two amplitudes $A^{*}$ and $B^{*}$, the phase $\operatorname{lag} \phi$ and, also, the dimensionless frequency of oscillation $\omega^{*}$. The point is that $\omega^{*}$ (directly related to $\omega$ ) is not known a priori in the forcing model (Eq. 6.3). So $\omega$ could be considered as an eigenvalue that allows the system (Eqs. 6.7a-6.7d) to have a solution. Therefore, for a prescribed design and operation configuration (fixed $m^{*}, \Omega, \mu, U^{*}, \zeta_{1}$ and $\zeta_{2}$ ), the solution procedure for Equations 6.7a-6.7d is iterative and can be described as follows:

Step 1

Algebraic manipulation of Equations 6.7c-6.7d yields 


$$
\begin{gathered}
\frac{B^{*}}{A^{*}}=\left(\frac{\Omega^{4}+\left(2 \zeta_{2} \omega^{*}\right)^{2}}{\left(2 \zeta_{2} \omega^{*}\right)^{2}+\left(\Omega^{2}-\mu \omega^{* 2}\right)^{2}}\right)^{\frac{1}{2}}, \\
\cos \phi=\frac{\Omega^{4}+4 \omega^{* 2} \zeta_{2}^{2}-\mu \omega^{* 2} \Omega^{2}}{\left(\left(\left(2 \zeta_{E} \omega^{*}\right)^{2}+\left(\Omega^{2}-\mu \omega^{* 2}\right)^{2}\right)\left(4 \omega^{* 2} \zeta_{2}^{2}+\Omega^{4}\right)\right)^{\frac{1}{2}}} .
\end{gathered}
$$

\section{Step 2} 6.8-6.9

Arbitrary $\omega_{i}^{*}$ and $A_{j}^{*}$ are chosen which allows for obtaining $B_{i j}^{*}$ and $\phi_{i j}$ from Equations

\section{Step 3}

$\omega_{i}^{*}, A_{j}^{*}, B_{i j}^{*}$ and $\phi_{i j}$ are inserted into Equations $6.7 \mathrm{a}-6.7 \mathrm{~b}$ and it is checked whether the residual is zero or not. If it is not, new values $\omega_{i+1}^{*}$ and $A_{j+1}^{*}$ are appropriately selected and the process is repeated until the residual of Equations $6.7 \mathrm{a}-6.7 \mathrm{~b}$ falls below some threshold value.

\subsection{Application case}

This Section deals with a typical application case that can be addressed using the analysis method developed in Section 6.3. This first case concerns a portable energy harvester meant to generate tens of milliwatts out of a small water stream for sensor power supply purposes. The actual volume occupied by the undeployed system is about $200 \mathrm{~cm}^{3}$ and its weight should be less than $250 \mathrm{~g}$, so that it can be carried on easily without much effort. This is representative of a broad class of mini energy harvesters that have been analyzed in depth in the literature. Table 6.1, that it is not intended to be a comprehensive literature search, shows some specific examples together with some of their characteristics:

The devices presented in Table 6.1 operate under different physics phenomena and under a wide variety of operating conditions, so they are not exactly comparable. Nevertheless, their power versus volume ratio is of the order of $0.01 \mathrm{~mW} / \mathrm{cm}^{3}$ to $1 \mathrm{~mW} / \mathrm{cm}^{3}$. As will be shown later, the device presented in this application case has a ratio of about $0.1 \mathrm{~mW} / \mathrm{cm}^{3}$, that is located somewhat in the middle of the range presented in Table 6.1. Water velocity is assumed to be $0.5 \mathrm{~m} / \mathrm{s}$ and the following parameters have been chosen for the primary resonator: $m_{1}=100 \mathrm{gr}, D=2 \mathrm{~cm}, L=10 \mathrm{~cm}$, see Figure 6.3. This is consistent with the resonator being a hollow cylinder manufactured on plastic. The cantilever, assumed to be manufactured on aluminum is $10 \mathrm{~cm}$ long, while its height and width will be chosen after $k_{1}$ is obtained. $m_{2}$, the translator mass of the generator, is assumed to be equal to the stator mass $m_{1}$. Typically, $c_{1}$ should be obtained from oscillation decay experiments of the actual cantilever. Since this is a theoretical study, results of $\zeta_{1}\left(\zeta_{1}=c_{1} / 2 \sqrt{k_{1} m_{1}}\right)$ for similar types of cantilever will be taken from the literature (2013). Now, the problem consists of obtaining the set of $k_{1}, k_{2}$ and $c_{2}$ that maximizes the system output power. This optimization problem can be straightforwardly solved by merely sweeping the three dimensional space of parameters $k_{1}, k_{2}$ and $c_{2}$ using the model presented in Section 6.2. That is the same concept as sweeping non-dimensional parameters 
Table 6.1: Characteristics of some mini energy harvesters studied in the literature.

\begin{tabular}{|c|c|c|c|c|c|}
\hline Reference & $\begin{array}{l}\text { Energy } \\
\text { Source }\end{array}$ & Generator type & $\begin{array}{l}\text { Power } \\
(\mathrm{mW})\end{array}$ & $\begin{array}{l}\text { Device } \\
\text { volume } \\
\left(\mathrm{cm}^{3}\right)\end{array}$ & $\begin{array}{l}\text { Power/Volum } \\
\left(\mathrm{mW} / \mathrm{cm}^{3}\right)\end{array}$ \\
\hline $\mathrm{Wu}(2013)$ & air, $10 \mathrm{~m} / \mathrm{s}$ & Piezoelectric & 2000 & 3340 & 0.60 \\
\hline $\begin{array}{l}\text { Abdelkefi } \\
\text { (2013a) }\end{array}$ & air, $5 \mathrm{~m} / \mathrm{s}$ & Piezoelectric & 10 & 22 & 0.45 \\
\hline Amini (2014) & air, $7.23 \mathrm{~m} / \mathrm{s}$ & Piezoelectric & 4 & 35 & 0.11 \\
\hline $\begin{array}{l}\text { Weinstein } \\
(2012)\end{array}$ & air, $2.5 \mathrm{~m} / \mathrm{s}$ & Piezoelectric & 0.2 & 620 & $310^{-4}$ \\
\hline $\begin{array}{l}\text { Hobeck } \\
(2012)\end{array}$ & air, $12 \mathrm{~m} / \mathrm{s}$ & Piezoelectric & 1 & 0.7 & 1.43 \\
\hline Jung (2011) & air, $4.5 \mathrm{~m} / \mathrm{s}$ & Electromagnetic & 370 & 3340 & 0.11 \\
\hline Yuan (2014) & $\begin{array}{l}\text { Prescribed vi- } \\
\text { bration }\end{array}$ & Piezoelectric & 0.1 & 9 & 0.01 \\
\hline $\begin{array}{l}\text { Makihara } \\
(2014)\end{array}$ & $\begin{array}{l}\text { Prescribed vi- } \\
\text { bration }\end{array}$ & Piezoelectric & $5.010^{-3}$ & 2 & $2.510^{-3}$ \\
\hline Harne (2012) & $\begin{array}{l}\text { Prescribed vi- } \\
\text { bration }\end{array}$ & Piezoelectric & $4.010^{-4}$ & 29 & $1.410^{-5}$ \\
\hline $\begin{array}{l}\text { Gambier } \\
(2012)\end{array}$ & $\begin{array}{l}\text { Prescribed vi- } \\
\text { bration }\end{array}$ & Piezoelectric & 0.49 & 3.5 & 0.14 \\
\hline $\mathrm{Yu}(2014)$ & $\begin{array}{l}\text { Prescribed vi- } \\
\text { bration }\end{array}$ & Piezoelectric & 28 & 1131 & 0.02 \\
\hline
\end{tabular}

$U^{*}, \Omega$ and $\zeta_{2}$ from Section 6.4. For each pair of $\left(\Omega, \zeta_{2}\right)$ values, a maximum efficiency is achieved in the domain of $U^{*}$, in other words, there is a reduced velocity $\left(U_{\text {max }}^{*}\right)$ in which the maximum efficiency $\left(\eta_{\max }\right)$ is located. Once this search has been performed, it is found that the optimum generator parameters are $k_{1}=295 \mathrm{~N} / \mathrm{m}, k_{2}=117 \mathrm{~N} / \mathrm{m}$ and $c_{2}=0.17 \mathrm{Ns} / \mathrm{m}$. The height and width of the cantilever were chosen to be $2 \mathrm{~cm}$ and $0.1 \mathrm{~cm}$ respectively. In this case the output power is $22 \mathrm{~mW}$. The peak to peak amplitude of the oscillation is found consistently from the solution of Equations $6.7 \mathrm{a}-6.7 \mathrm{~d}$ and it is equal to $2.3 \mathrm{~cm}$. That is, the semi amplitude is $1.2 \mathrm{~cm}$ that is smaller by a factor of 8.6 than the cantilever length so the assumption of one-dimensional motion is reasonably met. The conversion efficiency based on the sweeping area of the resonator (not on its diameter) is $8 \%$. Now, it is important to assess the robustness of system behavior when off design conditions are met. This has been done by computing the output power and the conversion efficiency of the selected design for a range of incident flow velocities. This is done in Figure 6.5a for the output power, and in Figure 6.5b for the conversion efficiency.

What Figures $6.5 \mathrm{a}$ and $6.5 \mathrm{~b}$ show is that both power and conversion efficiency do not deteriorate critically in a band of $5 \%$ of the design velocity.

Now, one of the advantages of having a reasonably simple theoretical model is that different types of optimization can be performed. For example, in the application case that has been presented so far, the device is optimized for a single velocity. However, when thinking about practical applications it is more sensible to design for a configuration meant to generate power 


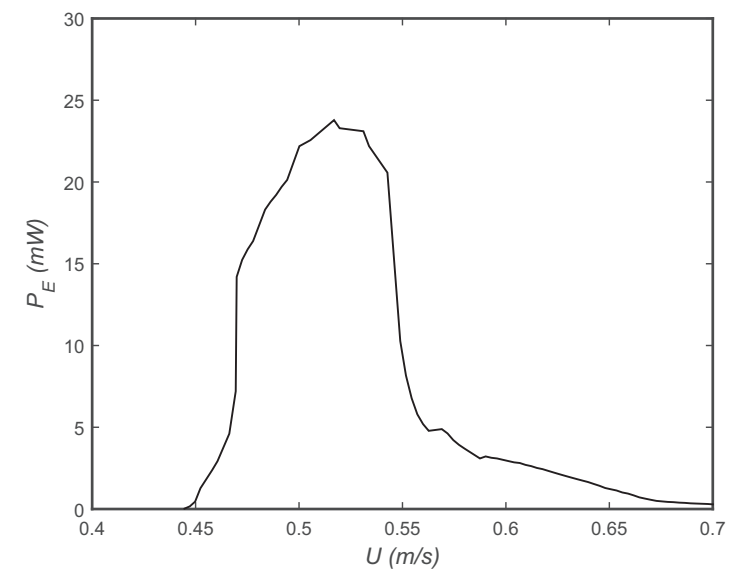

(a)

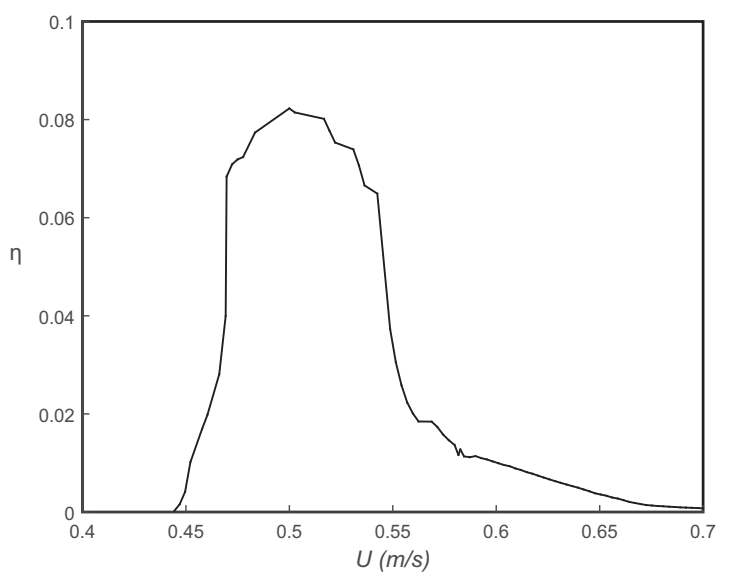

(b)

Figure 6.5: (a) Output power and (b) conversion efficiency versus incoming flow velocity for the selected optimum design.

in a range of flow velocities characterized by some utilization factor. For example, keeping the basic resonator configuration of the previous example $\left(m_{1}=100 \mathrm{gr}, m_{2}=100 \mathrm{gr}, D=2 \mathrm{~cm}\right.$, $L=10 \mathrm{~cm}$ ) the following problem (application case 2) is posed:

- To find the generator (defined by $k_{1}, k_{2}$ and $c_{2}$ ) that maximizes power in the range from 0.3 to $0.7 \mathrm{~m} / \mathrm{s}$ with the following utilization factors (UF): $U=0.3 \mathrm{~m} / \mathrm{s} F U=0.1, U=0.4$ $\mathrm{m} / \mathrm{s} F U=0.2, U=0.5 \mathrm{~m} / \mathrm{s} F U=0.4, U=0.6 \mathrm{~m} / \mathrm{s} F U=0.2, U=0.7 \mathrm{~m} / \mathrm{s} F U=0.1$.

This is a typical multiobjective optimization problem that has been solved and whose solution is $k_{1}=491 \mathrm{~N} / \mathrm{m}, k_{2}=195 \mathrm{~N} / \mathrm{m}$ and $c_{2}=0.217 \mathrm{Ns} / \mathrm{m}$. If $k_{1}$ can be varied according to the incoming flow speed in order to maximize the accumulative power extraction (for example varying the length of the cantilever beam). Power and dimensions of the cantilevers are presented in Table 6.2, where $a_{1}, a_{2}$ and $a_{3}$ are their length, height and width (only $a_{2}$ is changed).

Table 6.2: Results of the multiobjective optimization $\left(c_{2}=0.217 \mathrm{Ns} / \mathrm{m}, k_{2}=195 \mathrm{~N} / \mathrm{m}\right)$.

\begin{tabular}{llllll}
\hline$U$ & $0.3 \mathrm{~m} / \mathrm{s}$ & $0.4 \mathrm{~m} / \mathrm{s}$ & $0.5 \mathrm{~m} / \mathrm{s}$ & $0.6 \mathrm{~m} / \mathrm{s}$ & $0.7 \mathrm{~m} / \mathrm{s}$ \\
\hline FU & 0.1 & 0.2 & 0.4 & 0.2 & 0.1 \\
Power & $<1 \mathrm{~mW}$ & $1.6 \mathrm{~mW}$ & $8.2 \mathrm{~mW}$ & $30.4 \mathrm{~mW}$ & $52.8 \mathrm{~mW}$ \\
$k_{1}$ & - & $168 \mathrm{~N} / \mathrm{m}$ & $302 \mathrm{~N} / \mathrm{m}$ & $461 \mathrm{~N} / \mathrm{m}$ & $521 \mathrm{~N} / \mathrm{m}$ \\
$a_{1}$ & - & $10 \mathrm{~cm}$ & $10 \mathrm{~cm}$ & $10 \mathrm{~cm}$ & $10 \mathrm{~cm}$ \\
$a_{2}$ & - & $1 \mathrm{~cm}$ & $1.7 \mathrm{~cm}$ & $2.6 \mathrm{~cm}$ & $3 \mathrm{~cm}$ \\
$a_{3}$ & - & $0.1 \mathrm{~cm}$ & $0.1 \mathrm{~cm}$ & $0.1 \mathrm{~cm}$ & $0.1 \mathrm{~cm}$ \\
Efficiency & - & 0.011 & 0.030 & 0.065 & 0.071 \\
\hline
\end{tabular}

It was found in the optimization process that the optimum design point was for a velocity of $0.65 \mathrm{~m} / \mathrm{s}$. At $0.3 \mathrm{~m} / \mathrm{s}$, the output power was less than $1 \mathrm{~mW}$ and it was considered to be not 
practical. Regarding the different sizes of the cantilever, the user, in a actual situation, would carry on the system itself and a set of cantilevers (very light and easy to pack) and would select the most appropriate one for the encountered stream conditions.

Now, it is relevant to present the actuation maps of the system to see how they influence its design. This is done, first, in Figures $6.6 \mathrm{a}$ and $6.6 \mathrm{~b}$ where the maximum attainable efficiency is presented as a function of $\zeta_{2}\left(\zeta_{2}=c_{2} / 2 \sqrt{k_{1} m_{1}}\right)$ and $\Omega\left(\Omega=\sqrt{(}\left(k_{2} / k_{1}\right)\right)$. It could be observed in Figure 6.6a that for a given value of $\Omega$, the same efficiency can be obtained for two different values of $c_{2}$. Because of generator design considerations, it is important to choose the value that yields the lower value of $c_{2}\left(\zeta_{2}\right)$ and reachable values of $k_{2}$ and $k_{1}(\Omega)$. This is the reason why this region is expanded in Figure 6.6b.
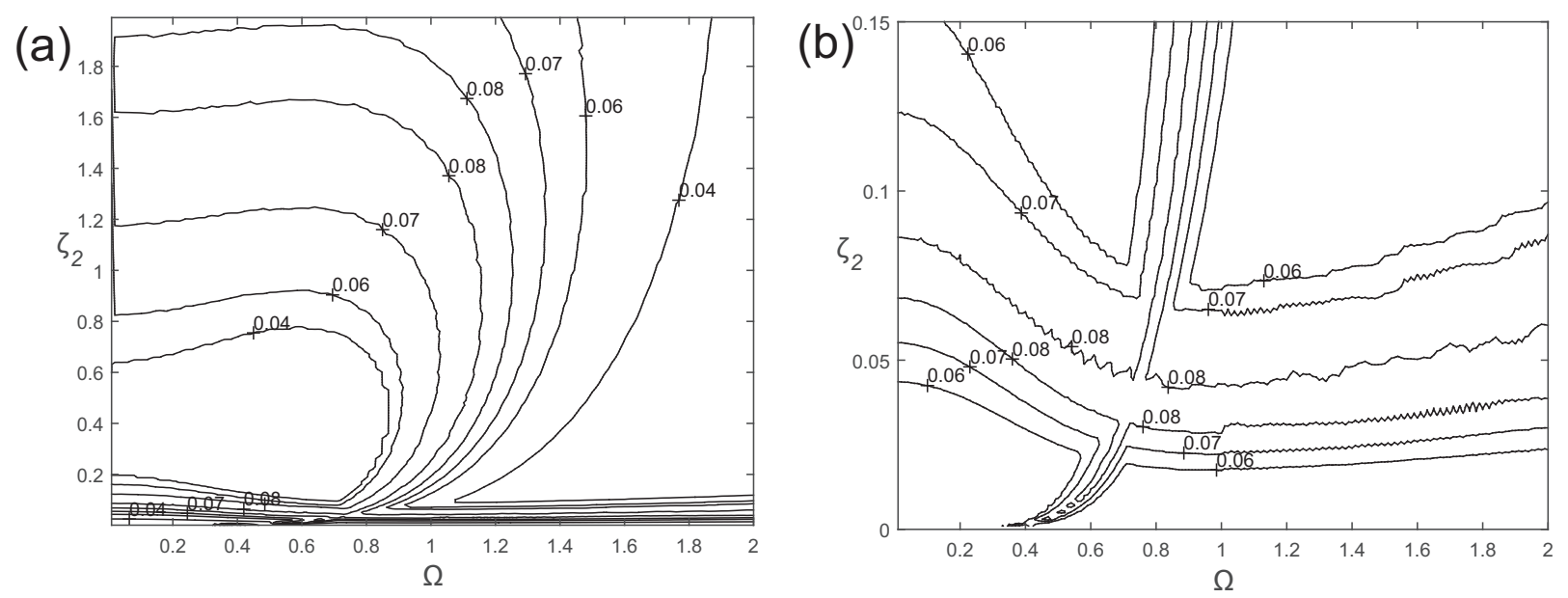

Figure 6.6: (a) Maximum attainable efficiency as a function of $\zeta_{2}$ and $\Omega$; (b) expended plot of Figure 6.6a in the region where branching occurs.

The value of $U^{*}\left(U^{*}=U_{\infty} / D \sqrt{k_{1} / m_{1}}\right)$ that maximizes the efficiency for any given pair of values $\zeta_{2}$ and $\Omega$ is presented in Figure 6.7.

Then, the design process to select $k_{1}, k_{2}$ and $c_{2}$ for any set of given $U_{\infty}, D, L, m_{1}, m_{2}$ and $\zeta_{1}$ is as follows:

- Step 1: A pair of $\left(\zeta_{2}, \Omega\right)$ is selected in Figure 6.6a (or 6.6b) in the region of maximum efficiency. This yields a relation between $c_{2}$ and $k_{1}\left(\zeta_{2}\right)$ and another one between $k_{2}$ and $k_{1}(\Omega)$.

- Step 2: For the selected pair of $\left(\zeta_{2}, \Omega\right)$ Figure 6.7 delivers the value of $U^{*}$ that yields the maximum efficiency. In practice this is a third relation (for $k_{1}$ ) that together with the two previous relations closes the problem.

The performance maps shown in Figure 6.6 and 6.7 allow, also, for the generation of design laws for optimal system of the base cylinder resonator, which consists of obtaining the cantilever dimensions and rigidity $\left(k_{1}\right)$, the translator spring constant $\left(k_{2}\right)$ and the amount of generator 


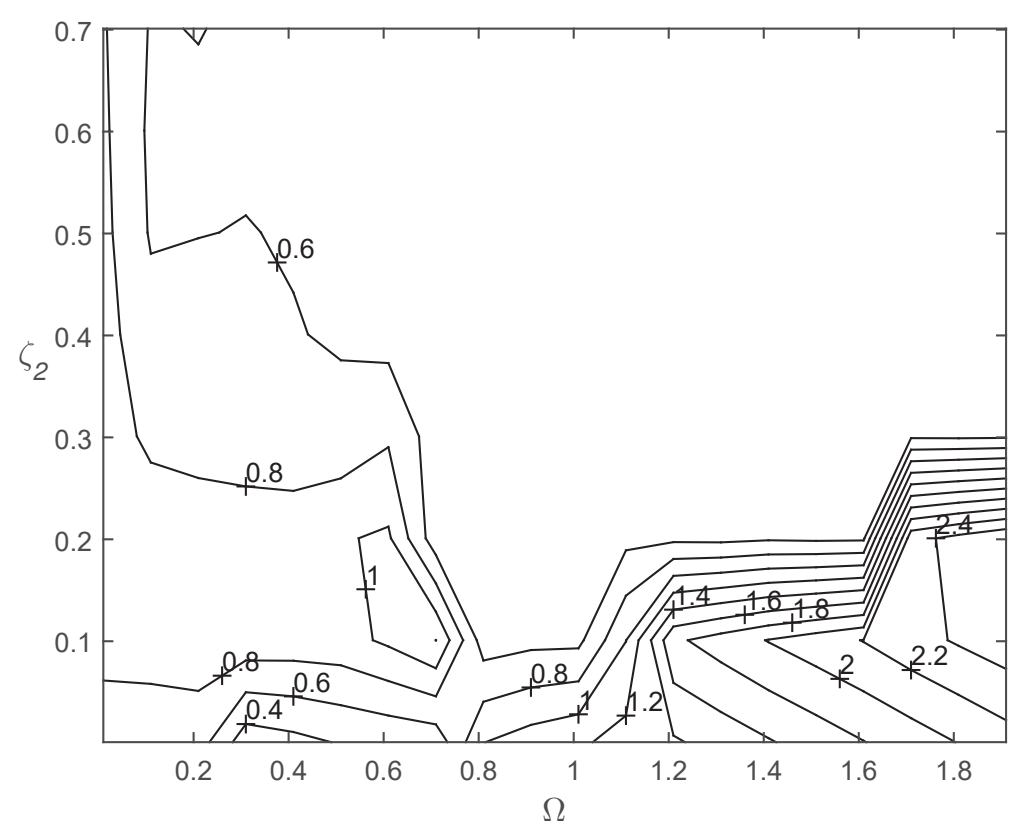

Figure 6.7: Value of $U^{*}\left(U_{\max }^{*}\right)$ that maximizes efficiency as a function of $\zeta_{2}$ and $\Omega$.

linear damping parameter $\left(c_{2}\right)$. The masses and dimensions of base cylinder plus the stator remain constant as hypothesis, such as $m_{1}, m_{2}, D$ and $L$. This could be done following the branches of Figure 6.6a (and 6.6b) that yield the maximum efficiency. However, because of practical design constraints of available generators, only the lower branch of Figure $6.6 \mathrm{~b}$ will be accounted for, and its associate $U^{*}$ plot (Figure 6.7). When moving along this line of maximum efficiency, the following relations could be written.

$$
\begin{gathered}
k_{1}=\left(\frac{U^{2} m_{1}}{D^{2}}\right) \frac{1}{\bar{U}^{* 2}}, \\
k_{2}=\left(\frac{U^{2} m_{1}}{D^{2}}\right) \frac{\bar{\Omega}}{\bar{U}^{* 2}}, \\
c_{2}=2 \frac{U m_{1}}{D} \frac{\overline{\zeta_{2}}}{\bar{U}^{*}},
\end{gathered}
$$

where overbar denotes the values of maximum efficiency in the performance maps. Relations 6.10-6.12 can be re-written in terms of new dimensionless parameters $\left(\lambda_{1}, \lambda_{2}\right.$ and $\left.\lambda_{3}\right)$ as follows:

$$
\begin{aligned}
& \lambda_{1}=\frac{k_{1} D^{2}}{U^{2}} m_{1}=\frac{1}{\bar{U}^{* 2}}, \\
& \lambda_{2}=\frac{k_{2} D^{2}}{U^{2}} m_{1}=\frac{\bar{\Omega}}{\bar{U}^{* 2}}
\end{aligned}
$$




$$
\lambda_{3}=\frac{c_{2} D}{2 U m_{1}}=\frac{\overline{\zeta_{2}}}{\bar{U}^{*}}
$$

Now the plots $\lambda_{2}$ versus $\lambda_{1}$ and $\lambda_{3}$ versus $\lambda_{1}$ are presented in Figures $6.8 \mathrm{a}$ and $6.8 \mathrm{~b}$ respectively. The actual results are plotted with circles, while the approximating curves are plotted with solid lines. In particular,

$$
\begin{gathered}
\lambda_{2}=2.3+0.12 \lambda_{1}, \\
\lambda_{3}=17 \lambda_{1}^{-4} .
\end{gathered}
$$

Those are the actual design laws of the optimal design. Using the definition of the $\lambda_{i}$ parameters, and considering $D=0.02 \mathrm{~m}$ and $m_{1}=0.1 \mathrm{Kg}$ :

$$
\begin{gathered}
\frac{k_{2}}{U^{2}}=575+0.12 \frac{k_{1}}{U^{2}}, \\
\frac{0.2 c_{2}}{2 U}=17\left(\frac{250 U^{2}}{k_{1}}\right)^{4} .
\end{gathered}
$$

That is, these two relations 6.18 and 6.19 represent the design relations existing between the governing parameter when an optimum design is sought. In particular, relation 6.19 can be re-written introducing a constant $\alpha$ as

$$
\frac{c_{2} k_{1}^{4}}{U^{9}}=\alpha
$$

At the branch of maximum efficiency, it is found after resolving the oscillation amplitude is $0.58 D$. Then, the incoming power per unit time that affects the harvesting system is:

$$
\frac{\frac{1}{2}(1 \times L \times(1.16 D+D)) 10^{3} U^{2}}{1 / U} \frac{J}{s}
$$

Then, since the optimum efficiency is 0.08 , the actual output power, $W_{\text {out }}$, is obtained multiplying Expression 6.21 by 0.08 , which yields:

$$
W_{\text {salida }}=86 L D U^{3}
$$




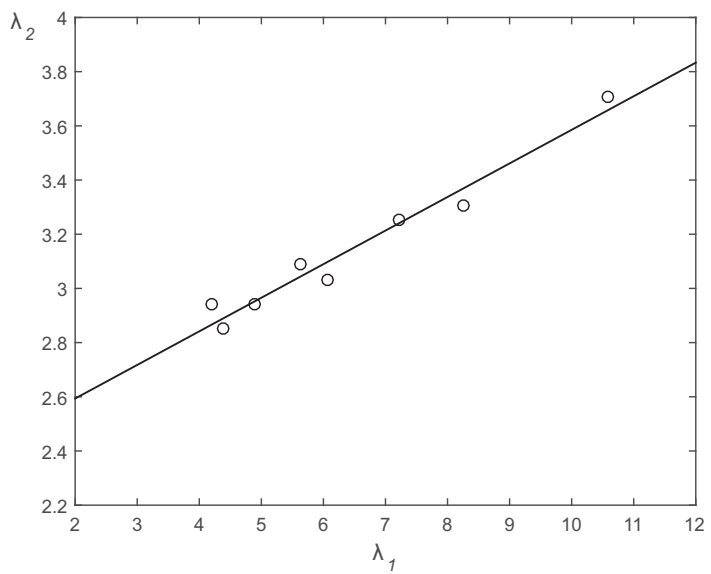

(a)

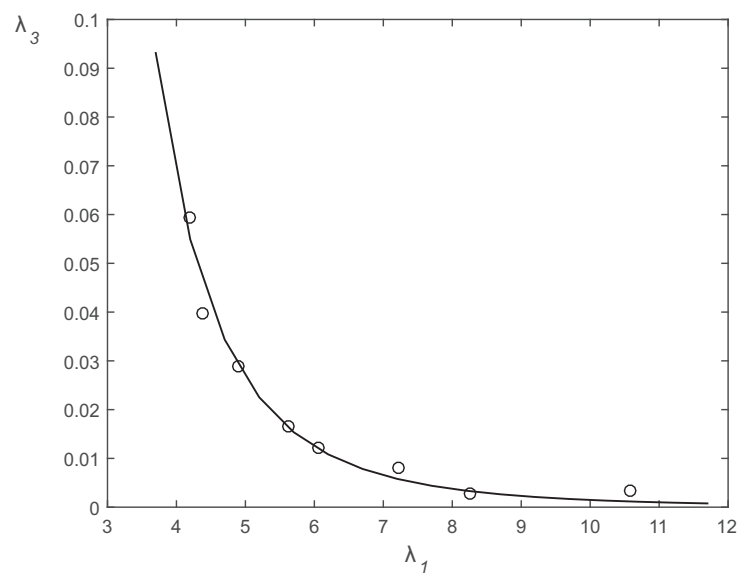

(b)

Figure 6.8: (a) Design curves $\lambda_{2}$ in terms of $\lambda_{1}$ for $m^{*}=2.5$ and $\mu=1$. (b) Design curves $\lambda_{3}$ in terms of $\lambda_{1}$ for $m^{*}=2.5$ and $\mu=1$.

\subsection{Conclusions}

A theoretical model has been proposed for the coupling between a VIV cylindrical resonator and its associated linear translator electromagnetic generator. The closure of the model comes via the implementation of experimental hydrodynamic forces maps which guarantees that the model provides realistic results. Based on this model, it is found that the maximum hydrodynamic to mechanical to electrical conversion efficiency (based on the VIV resonator oscillation amplitude) is $8 \%$. For a resonating cylindrical prism whose diameter and length are $2 \mathrm{~cm}$ and $10 \mathrm{~cm}$ respectively. This means that the output power would be in the range from $20 \mathrm{~mW}$ to 160 $\mathrm{mW}$ for water stream velocities spanning from $0.5 \mathrm{~m} / \mathrm{s}$ to $1 \mathrm{~m} / \mathrm{s}$. In practice, this should be enough to supply power to sensor type systems under field conditions. It has also been shown that the theoretical model can be readily used for multiobjective optimization purposes that are closer to an actual application than the case in which a single operating point is optimized. Additionally, regarding practical applications, it is to be said that the proposed system presents two distinct advantages. The first one is that the generator is above the water surface which means that water tightness concerns need not to be addressed, thereby simplifying the design and reducing the cost. The second one is that when operating under not necessarily gentle field conditions, the user may adapt to the water current velocity by simply selecting the appropriate cantilever. In this way, optimum operating conditions could be achieved with a high degree of simplicity. The theoretical model has also allowed for the generation of the design/performance maps of the system. In particular, the design laws that governing parameters have to follow where maximum energy conversion efficiency is sought are presented. These laws show (and this is quite apparent on Relation 6.20) that the design branch leading to maximum efficiency is most sensitive on the incoming water flow velocity. In an actual field application in which water velocity may vary but cylinder diameter cannot be changed, doubling the velocity implies multiplying roughly by 5 the stiffness constant of the supporting cantilever. 


\section{Chapter 7}

\section{Conclusions and future work}

\subsection{Conclusions and resume of Chapters}

In keeping with the broad scope of bluff body Flow-induced vibration (FIV) dynamics and energy transfer, this Thesis has attempted to shed light on a wide variety of related issues. This research consists of theoretical, analytical and experimental investigations of two perspectives of Flow-induced vibrations: fundamental studies of the FIV phenomenology and practical applications of FIV in energy harvesting. Better understanding, modelling, designs and efficiency enhancement techniques have been presented and discussed. The work carried out so far can be summarized as follows and some further research works are proposed.

- Transverse flow-induced oscillations of a square prism at low mass ratio $\left(m^{*}=2.2-14.4\right)$ and low mechanical damping have been experimentally studied in Chapter 2. The experimental set-ups to highlight are the water channel and the elastic flow-induced oscillating body. The flow-induced oscillating body, submerged in the water channel, is a rigid square cross-section prism fixed to an elastic system made of dual-parallel metallic flexor blades connected by rigid blocks at each end. By this way the square-section prism is free to oscillate in the normal direction to the flow, and at the same time, the elastic system presents linear stiffness and mechanical damping.

Body displacement and acceleration were recorded for each configuration in two minutes by a laser vibrometer and an accelerometer respectively. The recorded oscillations could be described by its amplitude and the main component of frequency. The functional dependence between the steady-state amplitude and frequency of oscillations with reduced velocity has been characterized. Within the studied ranges, the amplitude of oscillations increases with the reduced velocity monotonically. Concerning the effects of the mass ratio (five $m^{*}$ 's are tested) it has been observed that, as the mass ratio decreases, oscillations start at a lower reduced velocity; moreover, the constant of proportionality between reduced velocity and amplitude of oscillations depends on the mass ratio. A renormalization plot (a convergence of experimental data for different mass ratios) appears when steady-state amplitude is plotted against the "true" reduced velocity $V^{*}$, defined as the inflow velocity normalized by the characteristic length of the cross-section $(D)$ and the frequency of oscillations. Oscillations seem to start at a fixed value of the "true" reduced velocity. This value seems to be very similar to $\mathrm{St}^{-1}$, where $\mathrm{St}$ is the Strouhal number of vortex shedding for the square cylinder at rest.

Regarding the dynamics of the system, the phase lag between oscillations and fluid force is close to zero even for large values of reduced velocity. This suggests the non-triviality of the limit of applicability of the Quasi-steady theory. This limit seems to be much 
more complex than its classical definition in terms of reduced velocity $U^{*}$ stated in the literature to date. Taking into account experimental data, it has been found that the phase angle depends not only on the reduced velocity $U^{*}$ but also on the mass ratio $m^{*}$ as well as the mechanical damping $\zeta$. Increasing these three parameters leads to oscillations approaching Quasi-steady conditions. Finally, concerning the steadiness of oscillations, it has been observed that the amplitude of oscillations varies with time when vortex shedding effects are strong, for true reduced velocity values in the range of $V^{*}=7.7-13$. In this regime, any sinusoidal description of the dynamics of the square cylinder (i.e. amplitude and frequency of oscillations) and flow quantities (i.e. fluid force, phase lag) does not seem to be appropriate.

- A time delayed Quasi-steady theoretical model of Transverse Galloping has been developed. The theoretical model is based on the introduction of a time delay, which is only a function of the static empirical coefficients used in the traditional Quasi-steady model. Previous experimental results (both in free and forced vibrations) match closely with those given by the theoretical model. In addition, this model helps to explain experimental results which are in disagreement with predictions made using Quasi-steady theory. The new model contemplates a strong dependency on $m^{*}$ of the frequency of oscillations, as well as two different behaviours (branches) in the dependence of the steady-state normalized amplitude of oscillations $A^{*}$ with the reduced velocity. The model also helps to propose a Quasi-steady criterion where the threshold for the reduced velocity depends on the cross-section geometry, mass ratio and damping. The model developed in 3 may also explain the lack of agreement observed in the literature to establish this criterion. The model could be valid for design purposes and for estimating the onset of the Galloping oscillations and also the magnitude of oscillations.

- The aeroelastic Galloping of a piezo-cantilever with a prism attached on its tip has recently attracted the attention of several researchers as a way to harvest energy from an airstream. This arrangement is not entirely analogous to that of classical Transverse Galloping (TG) since the instantaneous attitude of the galloping body (prism) with respect to the incident flow depends not only on the velocity of the galloping body and wind speed (like in TG), but also on the rotation angle at the cantilever free end. A new governing parameter emerges, namely the ratio of the cross-section length of the prism to the beam length $\delta$, and whose effects on the galloping dynamics and power output have been studied. To this end, a theoretical model has been developed considering the influence of $\delta$. The analytical resolution of the formulation has been compared favourably with experimental results of Zhao et al. (2013). These analytical results state that the normalized amplitude of oscillations $A^{*}$ for cantilever Galloping is lower than that of pure Transverse Galloping, tending to an asymptotic finite value for a large reduced velocity $U^{*}$. Conversely, the normalized frequency of oscillations is larger than the value of unity predicted by the traditional TG model (. Translating these results to power extraction, it has been found that the electrical power output depends significantly on $\delta$ : Devices with lower values of $\delta$ harvest less power at a reduced velocity.. Another negative impact for energy harvesting due to the cantilever effect is that the output power tends asymptotically to an finite value for large values of reduced velocity $U^{*}$, whereas in pure TG the power grows with $U^{*}$ without reaching any limit. . It has also been observed that reduced velocity values for both, the begin of galloping oscillations and those for attaining maximum efficiency, 
depend on the value of delta. Correct application of observed results will be of paramount importance during the development of any real-world installation which is intended to operate at highest possible efficiency.

- A theoretical study is presented concerning power extraction via VIV of a circular cylinder in a dual-mass configuration. The dual mass system is modelled as a simplified two-degrees-of-freedom mechanical system where fluid forces on the circular cylinder are taken out of experimental data obtained from forced vibration tests. It is shown that power extraction can be optimized in certain reconfiguration scenarios if dual mass parameters are chosen appropriately. System behaviour has been characterised as a function of the governing parameters, and performance charts have been presented for parameter selection purposes in an engineering environment. The analysis has shown that under an ideal situation (when mechanical losses are zero $\zeta_{1}=0$ ) the theoretical maximum efficiency cannot be enhanced by using a dual-mass system, whereas if $\zeta_{1} \neq 0$, the maximum efficiency with respect to the single mass configuration can be improved if the dual mass parameters, namely the secondary mass $(\mu)$ and the stiffness between masses $(\Omega)$, are chosen appropriately. The dual-mass system also provides a more broadband response in terms of reduced velocity. From a fundamental point of view, the analysis carried out sheds light onto the mechanism by which the dual mass effect can be beneficial. The key idea is that, under equivalent configurations, the overall power dissipated is the same in the dual mass configuration than in the single mass one. But, when properly designed, the power dissipated-harvested at the generator in the dual mass configuration is increased proportionally in terms of amplitudes with respect to the power dissipated by mechanical losses, since oscillations of the dual mass are enhanced with respect to the oscillations of the main body. From a practical point of view, the analysis provides design charts to select dual-mass parameter, namely $\mu$ and $\Omega$. Taking this into account, a more efficient energy extracting device is designed taking advantage of the dual-mass effect, from a surrounding flow by VIV of a circular cylinder. Finally, despite of the approximation to the actual VIV problem, the results presented in Chapter 5 are believed to be representative and helpful to design a device to extract energy from VIV in an efficient way.

- Taking advantage of the two-way mass interaction formulation detailed in the Chapter 5, the dual-mass equations have been applied to a theoretical study of the coupling between a VIV cylindrical resonator and its associated linear electromagnetic generator. The dominant masses of the mentioned formulation are the stator and translator of the generator. In addition, a realistic milliwatt-energy harvesting device has been designed and its performances discussed. The rationale to carry out the study is the fact that in these types of configurations there is a two-way interaction between the moving parts in such a way that their motions influence each other simultaneously, thereby affecting the energy actually harvested. It is believed that a theoretical model can shed some light on the nature of the interaction and, at the same time, provide design laws that can be used for practical design and optimization purposes, before resorting to complementary numerical simulations. It has been found that the proposed configuration has a maximum hydrodynamic to mechanical to electrical conversion efficiency (based on the VIV resonator oscillation amplitude) of $8 \%$, for a cylindrical resonator $10 \mathrm{~cm}$ long with a $2 \mathrm{~cm}$ diameter, this translates into an output power of 20 to $160 \mathrm{~mW}$ for water stream velocities in the range from 0.5 to $1 \mathrm{~m} / \mathrm{s}$. In practice, this should be enough to supply power to 
sensor type systems under field conditions. It has also been shown that the theoretical model can be readily used for multiobjective optimization purposes that are closer to an actual application than the case in which a single operating point is optimized.

\section{$7.2 \quad$ Future works}

This section presents some recommendations for future work.

Taking advantage of the possibilities that low damping, low mass ratio and low turbulence recirculating water channel experimental test set-up brought, several parameter-variation effects could be tested. Specifically, according to the conclusions obtained in Chapter 2, one can carry out experimental campaigns where higher damping values are presented $(\zeta \sim 0.01-0.1)$, in order to corroborate the influence of damping coefficient on the approach of Quasi-steady condition. Otherwise, in terms of the third involved parameter $U^{*}$ and taking into account the flow velocity limitations of the turbulence controlled water channels, one can just diminish the transverse-section length $D$ or the natural frequency $\omega_{N}$ of the prism (taking into account that changing mechanical properties of the experimental set-up, the values of mass ratio and damping coefficient could be modified). All this parameter space scanning will be used to reach a better understanding of the square-section prism low damping and low mass ratio Flow-induced Vibration problem.

In order to gain insight to the Fluid-structure Interaction dynamics and somehow validate the time delayed Quasi-steady theoretical model, flow-field visualization and/or numerical works may be required to relate the time-delayed Quasi-steady model, the hypothesis of time delay presented in Chapter 3 of the amount of time that the flow around the galloping body requires to adapt itself to the new position of the body, to the vortex shedding of the galloping body. For example, to correlate the vortex shedding of the galloping body to the vortex shedding of a static experiment. According to the time delay Quasi-steady model hypothesis, the flow is delayed around the body with respect to a quiescent body, which means that the flow path at each time frame does not resemble to its corresponded static angle. Otherwise, the flow path in question may corresponded to a different angle of attack in the static experiment.

In another vein, numerical simulations may be useful to get information in the parameter space when $m^{*}, \zeta, U^{*}, U_{0}^{*}$ or $A^{*}$ are low.

The analytical formulation proposed in Chapter 4 has been resulted very similar to the experimental values obtained by Zhao et al. (2013). Analytically, further models for cantilevered galloping piezoelectric micro-generators can be proposed concerning the interactions fluid to solid body, solid body to cantilevered piezoelectric sheets and piezoelectric sheets to electrical load, optimizing each one of these couplings. Experimentally, wind tunnel tests can be performed in order to build a consistent theory for designing of these autonomous microgenerators/micro-sensors.

Furthermore, this Thesis, so far, have focused on linear models of the body's mechanism (namely, damping and stiffness), leading to quite periodic oscillatory responses. Numerical simulation or experiments at water channels could be done with non-linear structural dynamics. 
Complex non-periodic or even chaotic regimes may take place.

In the current research, the power has been dissipated on purely resistive electrical resistances. The complexity level in the transducer models may be increased and other ways (like active switching techniques) may be investigated closing the gap with practical engineering applications.

In all cases studied, a single isolated body has been considered in an unbounded (or close to unbounded) uniform flow. The behaviour in confined flows or with several bodies should be investigated. For example, it could occur that under some cooperative configurations, two bodies extract more energy from a flow than those two same bodies working separately. Some initial experiments have already been carried out, yielding increases of up to $50 \%$ in the amplitude of oscillation of a galloping body situated in certain configurations downstream the wake of the primary body (with respect to an equivalent isolated galloping body). In addition, in real environments the incoming flow is expected to be non-uniform and its role should be addressed. In particular, shear layers are not uncommon (in shallow waters) with non-stationary mean velocity of the flow with medium to high levels of turbulence which have big influence on the dynamical response of FIV. 



\section{Bibliography}

Abdelkefi A, Hajj M R, Nayfeh A H 2013a Piezoelectric energy harvesting from transverse galloping of bluff bodies Smart Materials and Structures 22015014

Abdelkefi A, Yan Z, Hajj M R 2013b Modeling and nonlinear analysis of piezoelectric energy harvesting from transverse galloping Smart Materials and Structures 22025016

Adhikari S, Friswell M I, Inman D J 2009 Piezoelectric energy harvesting from broadband random vibrations Smart Materials and Structures 18115005

Akaydin, Elvin N, Andrepoulus Y 2010 Wake of a cylinder: A paradigm for energy harvesting with piezoelectric materials Experiments in Fluids 49 291-304

Akaydin H D, Elvin N, Andrepoulos Y 2012 The performance of a self-excited fluidic energy harvester Smart Materials and Structures 21025007

Ali M, Arafa M, Elaraby M 2013 Harvesting energy from Galloping oscillations Proceedings of the World Congress on Engineering vol III

Allen J J, Smits A J 2001 Energy Harvesting EEL Journal of Fluids and Structures 15 000-000

Amini Y, Emdad H, Farid M 2014 An accurate model for numerical prediction of piezoelectric energy harvesting from fluid structure interaction problems Smart Materials and Structures 23 095034

Anton S R and Sodano H A 2007 A review of power harvesting using piezoelectric materials (2003-2006) Smart Materials and Structures 16 R1-21

Assi G R S, Meneghini J R, Aranha J A P, Bearman P W, Casaprima E 2006 Experimental investigation of flow-induced vibration interference between two circular cylinders Journal of Fluids and Structures 22 819-827

Assi G R S 2009 Mechanisms for flow-induced vibration of interfering bluff bodies (PhD Thesis)

Assi G R S, Bearman P W, Meneghini J R 2010 On the wake-induced vibration of tandem circular cylinders: the vortex interaction excitation mechanism Journal of Fluids Mechanics 66112 365-401

Assi G R S, Bearman P W, Carmo B S, Meneghini J R, Sherwin S J, Willden R H J 2013 The role of wake stiffness on the wake-induced vibration of the downstream cylinder of a tandem pair Journal of Fluid Mechanics 718 210-245

Assi G R S, Bearman P W 2015 Transverse galloping of circular cylinders fitted with solid and slotted splitter plates Journal of Fluids and Structures 54 263-280

Barrero-Gil A, Sanz-Andrés A, Roura M 2009a Transverse galloping at low Reynolds numbers Journal of Fluids and Structures 7 1236-1242 
Barrero-Gil A, Sanz-Andres A, Alonso G 2009b Hysteresis in transverse galloping: The role of the inflecion points Journal of Fluids and Structures 24 1007-1020

Barrero-Gil A, Alonso G, Sanz-Andres A 2010 Energy harvesting from transverse galloping Journal of Sound and Vibration 329 2873-2883

Barrero-Gil A, Pindado S, Avila S 2012 Extracting energy from Vortex-Induced Vibrations: A parametric study Applied Mathematical Modelling 36 3153-3160

Bearman P W 1984 Vortex shedding from oscillating bluff bodies Annual Review Fluid Mechanics 16195222

Bearman P W, Gartshore I S, Mauli D J, Parkinson G V 1987 Experiments on flow-induced vibration of a square-section cylinder Journal of Fluids and Structures 1 19-34

Bearman P W, Luo C 1988 Investigation of the aerodynamic instability of a square-section cylinder by forced oscillations Journal of Fluids and Structures 2, 161-176

Bernitsas M, Raghawan K, Ben-Simon Y, Garcia E M H 2008 VIVACE (vortex induced vibration for aquatic clean energy): a new concept in generation of clean and renewable energy from fluid flow J. Offshore Mech. Art. Eng., ASME Trans. 130041101

Bibo A, Abdelkefi A, Daqaq M F 2015 Modeling and Characterization of a piezoelectric energy harvester under combined aerodynamic and base excitations Journal of Vibration and Acoustics ASME 137(3) 031017-12

Blevins R D 1990 Flow-Induced Vibrations Van Nostrand Reinhold, Amsterdam

Bokaian A R, Geoola F 1983 On the cross flow response of cylindrical structures Processding s- Institution of Civil Engineers 75 397-418

Bouclin D N 1977 Hydroelastic oscillations of square cylinders Master Thesis, University of British Columbia, Vancouver, BC, Canada

Bradshaw P, Pankhurst R C 1964 The design of low-speed wind tunnels Progress in Aeronautical Science 6 1-69

von Büren T, Mitcheson P D, Green T C, Yeatman E M, Homes A S, Tröster Gerhard 2006 Optimization of Inertial Micropower Generators for Human Walking Motion IEEE Sensors Journal vol 6, No 1

von Büren T, Tröster G 2007 Design and optimization of a linear vibration-driven electromagnetic micro-power generator Sensors and Actuators A 135 765-775

Burrow S G, Penrose L 2014 A 2 DOF vibration harvester for broadband and multi-frequency harvesting using a single electro-magnetic transducer Journal of Physics: Conference Series 557012031

Cammarano A, Burrow S G, Barton D A W, Carella A, Clare L R 2010 Tuning a resonant energy harvester using a generalized electrical load Smart Materials and structures 19055003 
Dai H L, Abdelkefi A, Javed U, Wang L 2015 Modeling and performance of electromagnetic energy harvesting from galloping oscillations Smart Materials and Structures 24045012

Den Hartog J P 1932 Mechanical Vibrations McGraw-Hill Book Company

Deng L, Wen Z, Zhao X, Yuan C, Luo G, Mo J 2014 High Voltage Output MEMS Vibration Energy Harvester in $d_{31}$ Mode With PZT Thin Film Journal of Microelectromechanical Systems vol 23 No 4

Doaré O, Michelin S 2011 Piezoelectric coupling in energy-harvesting fluttering flexible plates: linear stability analysis and conversion efficiency Journal of Fluids and Structures 27 1357-1375

El-Hami M, Glynne-Jones P, White N M, Hill M, Beeby S, James E, Brown A D, Ross J N 2001 Design and fabrication of a new vibration-based electromechanical power generator Sensors and Actuators A: Physical 92 (1-3) 335-342

Ertuk A, Vieira W, de Marqui C, Inman D 2010 On the energy harvesting potential of piezoaeroelastic systems Applied Physics Letters 96184103

Facchinetti M L, de Langre E, Biolley F 2004 Coupling of structure and wake oscillators in vortex-induced vibrations Journal of Fluids and Structures 19123

Fung Y C 1955 An Introduction to the Theory of Aeroelasticity New York:Wiley

Gabbai R D, Benaroya H 2005 An overview of modeling and experiments of vortex-induced vibration of circular cylinders Journal of Sound and Vibration 282-3 575-616

Galchev T, Kim H, Najafi K 2011 Micro Power Generator for Harvesting Low-Frequency and Nonperiodic Vibrations Journal of Microelectromechanical Systems vol 20, No 4

Gambier P, Anton S R, Kong N, Erturk A, Inman D J 2012 Piezoelectric, solar and thermal energy harvesting for hybrid low-power generator systems with thin-film batteries Measurement Science and Technology 23015101

Govardhan N R, Williamson C H K 2002 Resonance forever: existence of a critical mass and an infinite regime of resonance in vortex-induced vibration Journal of Fluid Mechanics 473 $147-166$

Govardhan N R, Williamson C H K 2004 Critical mass in vortex-induced vibration of a cylinder European Journal of Mechanics-B/Fluids 2317

Govardhan N R, Williamson C H K 2006 Defining the 'modified Griffin plot' in vortex-induced vibration: revealing the effect of Reynolds number using controlled damping Journal of Fluid Mechanics 561 147-180

Grouthier C, Michelin S, De Langre E 2013 Energy Harvesting by Vortex-Induced Vibrations in Slender Structures ASME 2013 32nd International Conference on Ocean, Offshore and Arctic Engineering, Volume 7: CFD and VIV V007T08A013

Harne R L 2012 Concurrent attenuation of, and energy harvesting from, surface vibrations: experimental verification and model validation Smart Materials and Structures 21035016 
Hartog J P D 1984 Mechanical vibrations New York: McGraw-Hill

Hemon P 1999 An improvement of the time delayed quasi-steady model for the oscillations of circular cylidners in cross-flow Journal of Fluids and Structures 13 297-307

Hobeck J D and Inman D J 2012 Artifical piezoelectric grass for energy harvesting from turbulence-induced vibration Smart Materials and Structures 21105024

Hover F S, Miller S N, Tryantafillou M S 1997 Vortex-induced of marine cables: experiments using force feedback Journal of Fluids and Structures 11 307-326

Hover F S, Techet A H, Triantafyllou M S 1998 Forces on oscillating uniform and tapered cylinders in cross-flow Journal of Fluid Mechanics 36397114

Hover F S, Davis J T, Triantafyllou M S 2004 Three-dimensionality of mode transition in vortex-induced vibrations of a circular cylinder European Journal of Mechanics B/Fluids 23 $29-40$

Jung H J, Lee S W 2011 The experimental validation of a new energy harvesting system based on the wake galloping phenomenon Smart Materials and Structures 20055022

Khalak A, Williamson C H K 1997 Investigation of relative effects of mass and damping in vortex-induced vibration of a circular cylinder Journal of Wind Engineering and Industrial Aerodynamics 69 341-350

Kinsey T and Dumas G 2008 Parametric Study of an Oscillating Airfoil in a Power-Extraction Regime AIAA Journal 466 1318-1330

Kluger J M, Moon F C, Rand R H 2013 Shape optimization of a blunt body Vibro-wind galloping Journal of Fluids and Structures 40 185-200

Lee J H, Bernitsas M M 2011 High-damping, high-Reynolds VIV tests for energy harnessing using the VIVACE converter Ocean Engineering 38 1697-1712

De Langre E 2008 Effects of wind on plants Annual Review of Fluid Mechanics 40 141-168

Liang J, Liao W 2011 Energy flow in piezoelectric energy harvesting systems Smart Materials and Structures 20015005

Luo S C, Bearman P W 1990 Predictions of fluctuating lift on a transversely oscillating squaresection Journal of Fluids and Structures 4 219-228

Mackowski A W, Williamson C H K 2013 An experimental investigation of vortex-induced vibration with nonlinear restoring forces Physics of Fluids 25087101

Makihara K, Yamamoto Y, Horiguchi C, Sakaguchi H, Fujimoto K 2014 An Innovative Controller to Increase Harvested Energy Journal of Physics: Conference Series 557012023

Mitcheson P D, Green T C, Yeatman E M, Holmes A S 2004a Architectures for VibrationDriven Micropower Generators Journal of Microelectromechanical Systems vol 13, No 3 
Mitcheson P D, Miao P, Stark B H, Yeatman E M, Holmes A S, Green T C 2004b MEMS electrostatic micropower generator for low frequency operation Sensors Actuators A, Phys. vol 115 No $2 / 3$ 523-529

Morse T L, Williamson C H K 2009 Prediction of vortex-induced vibration response by employing controlled motion Journal of Fluid Mechanics 634 5-39

Naudascher E, Rockwell D 2005 Flow-Induced Vibrations: An Engineering Guide Dover Civil and Mechanical Engineering

Nemes A, Zhao J, Lo Jacono D, Sheridan J 2012 The interaction between flow-induced vibration mechanisms of a square cylinder with varying angles of attack Journal of Fluid Mechanics 710 $102-130$

Nishi Y 2013 Power extraction from vortex-induced vibration of dual mass system Journal of Sound and Vibration 332 199-212

Nishi Y, Ueno Y, Nishio M, Rodrigues L A, Kokubun K 2014 Power extraction using flowinduced vibration of a circular cylinder placed near another fixed cylinder Journal of Sound and Vibration 333-10 2863-2880

Novak M, Tanaka H 1974 Effect of turbulence on galloping instabilities ASCE Journal of the Engineering Mechanics Division 100 27-47

Païdoussis M P, Price S J, de Langre E 2011 Fluid-Structure Interactions: Cross-Flow-Induced Instabilities Cambridge University Press

Parkinson G V, Smith J D 1964 The square prism as an aeroelastic non-linear oscillator Quaterly Journal of Mechanics and Applied Mathematics 17 225-239

Parkinson G V, Brooks N P H 1961 On the Aeroelastic Instability of Bluff Cylinders Journal of Applied Mechanics 282 252-258

Parkinson G V 1989 Phenomena and modelling of flow-induced vibrations of bluff bodies Progress in Aerospace Sciences 26-2 169-224

Peano F, Tambosso T 2005 Design and optimization of a MEMS electret-based capacitive energy scavenger Journal of Microelectromechanical Systems vol 14 No 3 429-435

Prince S J, Paidoussis M P 1984 A theoretical investigation of the fluid elastic stability of a single flexible cylinder surrounded by rigid cylinders Flow-induced vibrations, ASME Symposium vol 2

Radi A, Lo Jacono D, Sheridan J 2014 A device to achieve low Reynolds numbers in an open surface water channel Exp. Fluids 0723-4864 55

Raghavan K 2007 Energy extraction from a steady flow using vortex induced vibration University of Michigan, Thesis Dissertation

Saadon S, Sidek O 2011 A review of vibration-based MEMS piezoelectric energy harvesters Energy Conversion and Management 52 500-504 
Sanchez-Sanz M, Fernandez B, Velazquez A 2009 Energy-Harvesting Microresonator Based on the Forces Generated by the Karman Street Around a Rectangular Prism Journal of Microelectromechanical Systems vol 18 No 2

Sanchez-Sanz M, Velazquez A 2009 Vortex-induced vibration of a prism in internal flow Journal of Fluid Mechanics 641 431-440

Sari I, Balkan T, Kulah H 2010 An electromagnetic micro power generator for low-frequency environmental vibrations based on the frequency upconversion technique Journal of Microelectromechanical Systems vol 19 No 1

Sarpkaya T 1978 Fluid forces on oscillating cylinders ASCE Journal of Waterway , Port , Coastal, and Ocean Division 104 275-290

Sarpkaya T 2004 A critical review of the intrinsic nature of vortex-induced vibrations Journal of Fluids and Structures 19389447

Simpson J E 1972 Effects of the lower boundary on the head of a gravity current Journal of Fluid Mechanics 534 759-768

Sirohi J, Mahadik R 2011 Piezoelectric Wind Energy Harvester for Low-Power Sensors Journal of Intelligent Systems and Structures 222215

Sirohi J, Mahadik R 2012 Harvesting Wind Energy Using a Galloping Piezoelectric Beam Journal of Vibration and Acoustics 134(1) 011009

Sumner D 2010 Two circular cylinders in cross-flow: a review Journal of Fluids and Structures26 849-899

Tang X, Païdoussis M P, Jiang J 2009 Cantilevered flexible plates in axial flow: Energy transfer and the concept of flutter-mill Journal of Sound and Vibration 326 263-276

Tang X, Zuo L 2011 Enhanced vibration energy harvesting using dual-mass systems Journal of Sound and Vibration 330 5199-5209

Tang L, Zhao L, Yang Y, Lefeuvre E 2015 Equivalent circuit representation and analysis of Galloping-based wind energy harvesting IEEE/ASME Transactions on mechatronics 202

Vicente-Ludlam D, Barrero-Gil A, Velazquez A 2014 Optimal electromagnetic energy extraction from transverse galloping Journal of Fluids and Structures 51 281-291

Vicente-Ludlam D, Barrero-Gil A, Velazquez A 2015 Enhanced mechanical energy extraction from transverse galloping using a dual mass system Journal of Sound and Vibration 339 290-303

Violette R, de Langre E, Szydlowski J 2007 Computation of vortex-induced vibrations of long structure using a wake oscillator model: Comparison with DNS and experiments Computers, Structures 851134

Virgin L N 2000 Introduction to experimental nonlinear dynamics. A case study in mechanical vibration Cambridge 
Wang P H, Dat X H, Fang D M, Zhao X L 2007 Design, fabrication and performance of a new vibration-based electromagnetic micro power generator Microelectronics Journal vol 38 No 12 1175-1180

Weinstein L A, Cacan M R, So P M, Wright P K 2012 Vortex shedding induced energy harvesting from piezoelectric materials in heating, ventilation and air conditioning flows Smart Materials and Structures 21045003

Williamson C H K, Govardhan R 2004 Vortex-induced vibrations Annual Review Fluid Mechanics 36413455

Wu N, Wang Q, Xie X 2013 Wind energy harvesting with a piezoelectric harvester Smart Materials and Structures 22095023

Xu-Xu J, Barrero-Gil A, Velazquez A 2015 A theoretical study of the coupling between a vortex-induced vibration cylindrical resonator and an electromagnetic energy harvester Smart Materials and Structures 24115009

Xu-Xu J, Barrero-Gil A, Velazquez A 2016a Dual mass system for enhancing energy extraction from Vortex-Induced Vibrations of a circular cylinder International Journal of Marine Energy Accepted Manuscript

Xu-Xu J, Barrero-Gil A, Velazquez A 2016b Experimental study on transverse flow-induced oscillations of a square-section cylinder at low mass ratio and low damping Experimental Thermal and Fluid Science 74 286-295

$\mathrm{Xu}-\mathrm{Xu}$ J, Vicente-Ludlam D, Barrero-Gil A 2016c Theoretical study of the energy harvesting of a cantilever with attached prism under aeroelastic gallopin European Journal of Mechanics $B /$ Fluids Article in press

Yang Y, Zhao L, Tang L 2013 Comparative study of tip cross-sections for efficient galloping energy harvesting Applied Physics Letters 102064105

Yu M, Peng Y, Wang S, Fu J, Choi S B 2014 A new energy-harvesting device system for wireless sensors, adaptable to on-site monitoring of MR damper motion Smart Materials and Structures 23077002

Yuan T, Yang J, Song R, Liu X 2014 Vibration energy harvesting system for railroad safety based on running vehicles Smart Materials and Structures 23125046

Zhao J, Leontini J S, Lo Jacono D, Sheridan J 2014 Fluid-structure interaction of a square cylinder at different angles of attack Journal of Fluid Mechanics 747 688-721

Zhao L, Tang L, Yang Y 2013 Comparison of modeling methods and parametric study for a piezoelectric wind energy harvester Smart Materials and Structures 22125003

Zhou W, Penamalli G R, Zuo Lei 2012 An efficient vibration energy harvester with a multi-mode dynamic magnifier Smart Materials and Structures 21015014

Zhu Q, Z Peng 2009 Mode coupling and flow energy harvesting by a flapping foil Physics of Fluids 213 
Zhu Q 2012 Energy harvesting by a purely passive flapping foil from shear flows Journal of Fluids and Structures 34 157-169 